TE WHARE WĀNANGA O TE ŪPOKO O TE IKA A MĀUI

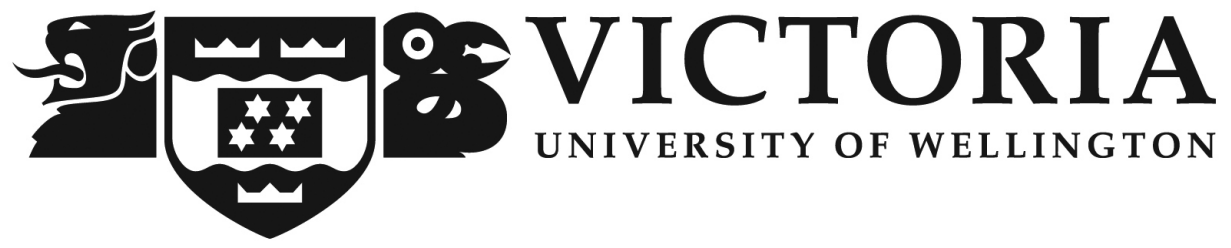

\title{
Exploring Diffuse Radio Emission in the Southern Sky
}

\author{
PhD Thesis
}

\section{Sara Shakouri}

Supervisor:

Associate Professor Melanie Johnston-Hollitt

A thesis submitted to the Victoria University of Wellington in fulfillment of the requirements for the degree of PhD in Physics. Victoria University of Wellington 

"Yesterday I was clever, so I wanted to change the world. Today I am wise, so I am changing myself."

Mawlana Jalal ad-Din Muhammad Balkhi (Rumi) 



\begin{abstract}
This thesis investigates currently observed correlations between the thermal and nonthermal (radio halos) components of galaxy clusters, and seeks to verify the reliability of the proposed radio halo scaling relations presented in the literature. It employs a twopronged approach: 1) a statistical examination of 15 galaxy clusters; and 2) detailed multi-wavelength analysis of individual objects of interest.

We first investigated radio data for 15 galaxy clusters drawn from the parent REXCESS sample observed with the ATCA at $1.4 \mathrm{GHz}$ to conduct a radio halo survey. Examination of available and re-processed low resolution images revealed cluster-scale diffuse objects in three clusters. One was a radio halo candidate in Abell 3888 (A3888), with the two remaining diffuse sources being radio relic candidates. Follow-up observations of the candidate clusters were performed in July and December 2011, and March 2012, with the upgraded ATCA (CABB). Radio observations with CABB in different array configurations were used to provide the required resolution and sensitivity to a wider range of angular scales to probe the candidate diffuse sources. Examination of the final CABB images confirmed the existence of the radio halo in A3888; however, the remaining candidates were found to be a head-tail galaxy and a very bright radio galaxy with extended emission. As this thesis presents some of the earliest CABB observations, new data reduction and imaging procedures were necessarily developed and presented here.
\end{abstract}

The statistical component of this thesis uses a halo sample obtained from the combined detection of this work and the literature to derive new correlations between the cluster observables and the radio halo power. The new correlation between the X-ray luminosity and radio halo power derived here is flatter than the previous correlation in the literature, suggesting that massive clusters may host lower power halos than previously thought. In addition, we derived the upper limits of the undetected power of possible radio halos for our non-halo clusters via injection of fake radio halos into the UV data. Our derived upper limits with respect to the anticipated halo powers according to the previous and new correlations and their interpretations are discussed in the thesis. The distribution of the combined upper limits (this work and the literature) compared to our new correlation shows no sign of the strong bi-modality found in the literature.

As previously mentioned, we detected a giant radio halo in A3888. We observed A3888 with the AAOmega spectrograph to infer the dynamics of the cluster. We measured the spectra of 254 galaxies within a $30^{\prime}$ radius from the core of A3888 and combined these data with the available literature redshifts in the region. We identified 71 member galaxies of A3888 and examined the density contours, which indicated that the distribution of the member galaxies is elongated along an east-west axis. This elongation might be indicative of dynamical interactions in the cluster; however, there is no 
statistically significant deviation from Gaussianity in the velocity data. We then carried out a Lee-Fitchett 3D substructure test and found that A3888 is bimodal and has two subgroups.

The head-tail galaxy mentioned earlier in one of the clusters was originally thought to be a radio relic candidate. Owing to available broadband polarimetric data and wellseparated jets of the head-tail galaxy, we investigated the magnetic field direction of the head-tail galaxy and conclude it is highly likely that a helical magnetic field is present in the jets.

We present the high-resolution images of our 15 clusters and create a catalogue of the detected sources. Finally, we discuss concerns with the current radio halo detections in the literature, and how radio halo surveys could be designed in the future to yield unbiased results. 


\section{Statement of Originality}

To the best of my knowledge, this thesis has not been previously accepted by any institution for the award of a degree or diploma and previously published materials were only used with due acknowledgements.

Victoria University of Wellington, New Zealand.

Signature

Sara Shakouri

Wellington, December 2015 



\section{Acknowledgements}

Firstly, I would like to express my sincere gratitude to my supervisor Associate Professor Melanie Johnston-Hollitt for her continuous support throughout my $\mathrm{PhD}$ studies. Especially for her patience, encouragement and immense knowledge and for letting me fulfil my dream to be a $\mathrm{PhD}$ student in astronomy. She has always supported me during the time I became a mother and when I was trying to fulfil my motherhood responsibilities during these years.

I would like to thank the staff of the ATCA; especially Dr Phil Edwards who kindly offered the ATCA green time. I would like to deeply thank Dr Robin Wark for her assistance with remote-observing, and Dr Jamie Stevens and Dr Mark Wieringa for their invaluable comments on data reduction and imaging in Miriad and CASA.

My sincere thanks also goes to the AAT staff for the AAOmega observations in service mode, and Dr Sarah Brough for her contribution to my AAOmega proposal. I would like to deeply thank Dr Michiel Brentjens for providing me the modified version of the RM synthesis code, and Dr George Heald for his useful comments regarding the RM synthesis code usage.

My sincere thanks goes to Dr Ana Laura Serra for providing the Caustics code and outlining the method and Dr Urvashi Rau from NRAO for her great comments on broadband imaging in CASA. I am grateful to Prof Larry Rudnick for his insightful comments on the RM synthesis and rotation measure. I thank Dr Siamak Dehghan, Dr Luke Hindson and Rowan Miller for their generous contributions to the data collection. My appreciation goes to Prof Geoff Bicknell and Dr Leif Godfrey for their insightful comments on toroidal magnetic fields in jets. I thank the Ministry of Business, Innovation and Employment for funding my attendance at the Radio Astronomy School in Narrabri, Australia, in September 2010. I am sincerely grateful to Victoria University of Wellington for providing my PhD scholarship, and the SKA organisation for my attendance at the China-Newzealand SKA Summer School in Shanghai in 2014.

Last but not the least, I would like to deeply appreciate my husband and my mother for their endless support during these years. I am grateful for their unconditional love and encouragement that have kept me moving forward during the difficult times. Finally, I would like to dedicate this thesis to my son, Ryan, who was born while this work was being conducted. I can't wait to talk to him about our Universe while we are observing planets. 



\section{Contents}

1 Radio Sources in Galaxy Clusters 1

1.1 Radio Galaxies . . . . . . . . . . . . . . . . . . . . . . . . 1

1.2 Diffuse Radio Sources in Clusters . . . . . . . . . . . . . . . . . . 4

1.2.1 Radio Halos . . . . . . . . . . . . . . . . . . . . . . . 4

1.2.2 Radio Relics . . . . . . . . . . . . . . . . . 6

1.2 .3 Mini Halos . . . . . . . . . . . . . . . . 8

2 Radio Halos: Theoretical Overview 9

2.1 Galaxy Clusters . . . . . . . . . . . . . . . . . . . . 9

2.1.1 Cluster Morphology _. . . . . . . . . . . . . . 10

2.2 Magnetic Field in Galaxy Clusters ．. . . . . . . . . . . . . . . . . 11

2.2.1 Faraday Rotation . . . . . . . . . . . . . . . . . 11

2.2.2 RM Observations of Clusters . . . . . . . . . . . . . . . . 13

2.3 Non-thermal Emission and Losses in Galaxy Clusters . . . . . . . . . . 14

2.3.1 Synchrotron Losses . . . . . . . . . . . . . . . . . . . . . . . 14

2.3.2 Inverse Compton Losses . . . . . . . . . . . . . . . . 15

2.3.3 Coulomb Losses . . . . . . . . . . . . . . . . . . 16

2.3.4 CRe Life-time . . . . . . . . . . . . . . . . . 16

2.4 Secondary Cosmic Ray Electrons in Clusters . . . . . . . . . . . . . . 17

2.5 Gamma Ray Emission in Clusters . . . . . . . . . . . . . . . . 18

2.6 Particle Acceleration in Clusters _. . . . . . . . . . . . . . . . 19

2.6.1 Fermi Acceleration Mechanisms . . . . . . . . . . . . . . . . 19

2.7 Radio Halo Generation Models . . . . . . . . . . . . . . . . . . . . 22

3 Data Reduction and Imaging of CABB Data 27

3.1 Australia Telescope Compact Array . . . . . . . . . . . . . . . . . . . 27

3.2 CABB Data Reduction . . . . . . . . . . . . . . . . . 28

3.3 Imaging of CABB data . . . . . . . . . . . . . . 32

3.4 Summary . . . . . . . . . . . . . . . . . 33

4 Radio Imaging of REXCESS Sub-sample 35

4.1 Radio Halo Observations in Galaxy Clusters . . . . . . . . . . . . . . . 35

4.2 The REXCESS Sample . . . . . . . . . . . . . . . . . . . . 36

4.3 Pre-CABB Observations and Low Resolution Images . . . . . . . . . 37

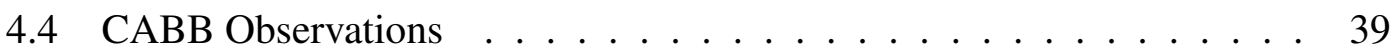


4.5 High Resolution Images $\ldots \ldots$. . . . . . . . . . . . . . . . 40

4.6 Source Detection . . . . . . . . . . . . . . . . . . . 41

4.7 REXCESS Cluster RXCJ0006.0-3443 . . . . . . . . . . . . . . 42

4.8 REXCESS Cluster RXCJ0049.4-2931 . . . . . . . . . . . . . . . 43

4.9 REXCESS Cluster RXCJ0145.0-5300 . . . . . . . . . . . . . . . 43

4.10 REXCESS Cluster RXCJ0211.4-4017 . . . . . . . . . . . . . . . . 44

4.11 REXCESS Cluster RXCJ0225.1-2928 . . . . . . . . . . . . . . 44

4.12 REXCESS Cluster RXCJ0345.7-4112 . . . . . . . . . . . . . . . 45

4.13 REXCESS Cluster RXCJ0616.8-4748 . . . . . . . . . . . . . . 46

4.14 REXCESS Cluster RXCJ0645.4-5413 . . . . . . . . . . . . . . . . 46

4.15 REXCESS Cluster RXCJ1236.7-3354 . . . . . . . . . . . . . . 47

4.16 REXCESS Cluster RXCJ2014.8-2430 . . . . . . . . . . . . . . . 47

4.17 REXCESS Cluster RXCJ2023.0-2056 . . . . . . . . . . . . . . 48

4.18 REXCESS Cluster RXCJ2129.8-5048 . . . . . . . . . . . . . . . . 49

4.19 REXCESS Cluster RXCJ2149.1-3041 . . . . . . . . . . . . . . . . . 49

4.20 REXCESS Cluster RXCJ2218.6-3853 . . . . . . . . . . . . 50

4.21 REXCESS Cluster RXCJ2234.5-3744 . . . . . . . . . . . . . . 51

4.22 Summary . . . . . . . . . . . . . . . . . 51

5 Diffuse Sources in the REXCESS sub-sample 83

5.1 The Radio Halo in Abell 3888 . . . . . . . . . . . . . . . . . 83

5.1.1 Radio Halo Properties in A3888 . . . . . . . . . . . . 83

5.1 .2 Integrated Spectral Index . . . . . . . . . . . . . . . 85

5.1.3 Power/Size Scaling Relation . . . . . . . . . . . . . . 85

5.1.4 Morphology of A3888: Literature Review . . . . . . . . . . . . 88

5.2 The Peculiar Diffuse Source in RXCJ0225.1-2928 . . . . . . . . . . . . 90

5.2.1 Stokes I and Polarisation Imaging . . . . . . . . . . . 90

5.2.2 Optical/IR Investigation of Cluster RXCJ0225.1-2928 _ . . . . 92

5.2.3 The Origin of Diffuse Emission in Cluster RXCJ0225.1-2928 . 94

5.3 Summary and Conclusion . . . . . . . . . . . . . . . . 97

6 Optical Analysis of Abell $3888 \quad 99$

6.1 Introduction . . . . . . . . . . . . . . . . . . . . . . 99

6.2 AAOmega Observation of A3888 . . . . . . . . . . . . . 101

6.3 Data Reduction and Redshift Determination . . . . . . . . . . . . . 103

6.4 Redshift Extraction Results . . . . . . . . . . . . . . . . . . . . . . 104

6.5 Spectroscopic Redshift Completeness . . . . . . . . . . . . . . . . . 108

6.6 Cluster Membership . . . . . . . . . . . . . . . . . . . . . . . . . . . . 109

6.6.1 DBSCAN Clustering Algorithm . . . . . . . . . . . 110 
6.6.2 Caustic Technique . . . . . . . . . . . . . . 113

6.7 Structures in the Main Population of A3888 . . . . . . . . . . . . 116

6.8 Substructure Test of A3888 . . . . . . . . . . . . . . . . . . 119

6.9 Foreground and Background Structures . . . . . . . . . . . . . 120

6.10 Morphological Content of A3888 . . . . . . . . . . . . . . . 125

6.10.1 Radio/Optical Connection Investigation . . . . . . . . . . 128

6.11 Summary and Conclusion . . . . . . . . . . . . . . . . 128

7 Radio Halo Statistics $\quad 131$

7.1 Introduction . . . . . . . . . . . . . . . . . . 131

7.2 Fake Radio Halo Injection . . . . . . . . . . . . . . . . . 133

7.3 Radio Halo Fraction . . . . . . . . . . . . . . . . . . . . 137

7.4 Radio / X-ray Luminosity: Correlation \& Bi-modality . . . . . . . . . . 139

7.5 Radio / SZ \& Radio / Mass Relations \& Bi-modality . . . . . . . . . . 142

7.6 Lack of a Halo in A3771 and A3404 . . . . . . . . . . . . . . . . . . 147

7.7 Summary and Discussion . . . . . . . . . . . . . . . . . 148

8 Magnetic Field in the Jets of SJ212919-505226 151

8.1 Introduction . . . . . . . . . . . . . . . . . 151

8.2 Radio Observations and Stokes I Imaging . . . . . . . . . . . . 152

8.3 Polarimetry . . . . . . . . . . . . . . . . . . 154

8.4 Spectral Index Map of SJ212919-505226 . . . . . . . . . . . . . . 157

8.5 Rotation Measure of SJ212919-505226 . . . . . . . . . . . . 158

8.6 Magnetic Field Direction in SJ212919-505226 . . . . . . . . . . . 163

8.7 Discussion . . . . . . . . . . . . . . . . . . . 164

8.8 Summary . . . . . . . . . . . . . . . . . 169

9 The Outlook $\quad 171$

9.1 Future Radio Halo Surveys . . . . . . . . . . . . . . . . . . . . 171

A The Southern REXCESS Radio Catalogue 196

B List of All Detetcted Radio Halos $\quad 212$

$\begin{array}{llr}\text { C } & \text { Morphology Estimators in X-ray Images } & \mathbf{2 1 8}\end{array}$

D AAOmega Redshift Measurements $\quad 220$ 


\section{List of Figures}

1.1 Example of an FRI radio galaxy . . . . . . . . . . . . . 2

1.2 Example of an HT galaxy . . . . . . . . . . . . . . . . . 3

1.3 An example of a radio halo in A3888 . . . . . . . . . . . . . . 4

1.4 Radio spectrum of Coma . . . . . . . . . . . . . . . . 5

1.5 Double radio relic in $\mathrm{A} 3667 \ldots \ldots \ldots \ldots$

2.1 The RM distribution of a sample of clusters . . . . . . . . . . . . 13

2.2 CRe life-time in different magnetic fields and different $\mathrm{n}_{e} \ldots \ldots 17$

2.3 Fermi Acceleration Mechanisms _ . . . . . . . . . . . . . 20

2.4 An overview of the energy gain and loss of CRs in galaxy clusters . . . 21

2.5 CRs life-time and diffusion time . . . . . . . . . . . . . . 22

3.1 Australia Telescope Compact Array (ATCA) . . . . . . . . . . . . . . . 27

3.2 Data reduction flow chart in MIRIAD for CABB . . . . . . . . . . . 29

3.3 Band-pass shape of the non-calibrated and calibrated PKS B1934-638 . 31

3.4 Calibration process in MIRIAD for CABB . . . . . . . . . . . . . 32

4.1 The $\mathrm{L}_{X} \mathrm{-Z}$ distribution of the REFLEX cluster sample . . . . . . . . . 37

4.2 Low resolution pre-CABB images of candidate diffuse sources . . . . . 53

4.3 Detected radio sources in RXCJ0006.0-3443 . . . . . . . . . . . . . . . 54

4.4 Postage stamps of extended sources in RXCJ0006.0-3443 . . . . . . . 55

4.5 Detected radio sources in RXCJ0049.4-2931 . . . . . . . . . . . . 56

4.6 Postage stamps of extended sources in RXCJ0049.4-2931 . . . . . . . . 56

4.7 Detected radio sources in RXCJ0145.0-530 . . . . . . . . . . . . 57

4.8 Postage stamps of extended sources in RXCJ0145.0-5300 . . . . . . . 58

4.9 Detected radio sources in RXCJ0211.4-4017 . . . . . . . . . . . . . . 59

4.10 Postage stamps of extended radio sources in RXCJ0211.4-4017 . . . . . 59

4.11 Detected radio sources in RXCJ0225.1-2928 . . . . . . . . . . . . 60

4.12 Postage stamps of extended radio sources in RXCJ0225.1-2928 . . . . . 61

4.13 Detected radio sources in RXCJ0345.7-4112 . . . . . . . . . . . . . 62

4.14 Postage stamps of extended radio sources in RXCJ0345.7-4112 . . . . . 63

4.15 Detected radio sources in RXCJ0616.8-4748 . . . . . . . . . . . . . 64

4.16 Postage stamps of extended radio sources in RXCJ0616.8-4748 . . . . . 65

4.17 Detected radio sources in RXCJ0645.4-5413 . . . . . . . . . . . . . 66

4.18 Postage stamps of extended sources in RXCJ0645.4-5413 . . . . . . . 67 
4.19 Detected radio sources in RXCJ1236.7-3354 . . . . . . . . . . . . . 68

4.20 Postage stamps of extended sources in RXCJ1236.7-3354 . . . . . . . . 69

4.21 Detected radio sources in RXCJ2014.8-2430 . . . . . . . . . . . . 70

4.22 Postage stamps of extended sources in RXCJ2014.8-2430 . . . . . . 71

4.23 Detected radio sources in RXCJ2023.0-2056 . . . . . . . . . . . 72

4.24 Postage stamps of extended radio sources in RXCJ2023.0-2056 . . . . . 73

4.25 Detected radio sources in RXCJ2129.8-5048 . . . . . . . . . . . . . 74

4.26 Postage stamps of extended radio sources in RXCJ2129.8-5048 . . . . . 75

4.27 Detected radio sources in RXCJ2149.1-3041 . . . . . . . . . . . 76

4.28 Postage stamps of extended sources in RXCJ2149.1-3041 . . . . . . . . 77

4.29 Detected radio sources in RXCJ2218.6-3853 . . . . . . . . . . . . 78

4.30 Postage stamps of extended sources in RXCJ2218.6-3853 . . . . . . . . 79

4.31 Detected radio sources in RXCJ2234.5-3744 . . . . . . . . . . . . . . 80

4.32 Postage stamps on extended radio sources in RXCJ2234.5-3744 . . . . 81

5.1 Radio halo in A3888 f . . . . . . . . . . . . . . . . . . 84

5.2 Integrated spectral index of halo in A3888 . . . . . . . . . . 86

$5.3 \mathrm{P}_{1.4}$ vs LLS plot . . . . . . . . . . . . . . . . . . 87

$5.4 \mathrm{P}_{1.4} \mathrm{vs} \mathrm{R}_{R H}$ plot $\ldots \ldots \ldots \ldots \ldots \ldots$

5.5 X-ray emission of the cluster RXCJ0225.1-2928 . . . . . . . . . . . 90

5.6 Magnetic field direction of the diffuse source in RXCJ0225.1-2928 . . . 91

5.7 Diffuse radio source in cluster RXCJ0225.1-2928 . . . . . . . . . . 92

5.8 Clusters around the diffuse radio emission in RXCJ0225-2829 . . . . . 92

5.9 Redshift histogram of RXCJ0225.1-2928 . . . . . . . . . . . . . 93

5.10 Galaxy distribution in RXCJ0225.1-2928 . . . . . . . . . . . . . 94

6.1 The optical fibre configuration in AAOmega . . . . . . . . . . . . . 102

6.2 Two examples of reduced spectra observed with the AAOmega . . . . . 105

6.3 Common objects between the AAOmega observations and the literature 106

6.4 The colour-coded redshift histogram of A3888 . . . . . . . . . . . 107

6.5 Cumulative spectroscopic completeness of A3888 . . . . . . . . . . 108

6.6 Cumulative redshift completeness in radial bins of $500 \mathrm{kpc}$ in A3888 . . 109

6.7 The sorted k-dist plot with $\mathrm{k}=6$. . . . . . . . . . . . . . . . . 112

$6.8 \sigma_{d i s}$ of the nodes along the hierarchical tree . . . . . . . . . . 115

6.9 The caustic profile of A3888 . . . . . . . . . . . . . 116

6.10 The isoline plot structures detected by DBSCAN method . . . . . . . 118

6.11 Distribution of member galaxies of cluster A3888 and structures $2 \& 3 \quad$. 120

6.12 Redshift histogram of A3888 candidate members . . . . . . . . . . . 121

6.13 Lee-Fitchett 3D substructure test of A3888 . . . . . . . . . . . . . . 122 
6.14 Spatial distribution of galaxies in the detected structures . . . . . . . . 123

6.15 The isoline plot of the galaxies in the field of A3888 . . . . . . . . . 124

6.16 Redshift histogram of detected galaxy clusters with the DBSCAN . . 125

6.17 Redshift distribution of Structure 7 . . . . . . . . . . . . . . 126

6.18 Distribution of galaxies in structure 9 . . . . . . . . . . . . . 127

6.19 Contour plot of the structure 9 . . . . . . . . . . . . . . . . . 128

6.20 The morphological fraction correlation with $\mathrm{L}_{X}$ and velocity dispersion 129

6.21 Radio/Optical connection in A3888 . . . . . . . . . . . . . . 130

7.1 Example of a halo injection in the cluster RXCJ2218.6-385 . . . . . . 135

7.2 Distribution of non-halo clusters on the $\mathrm{P}_{1.4}-\mathrm{L}_{X}$ plane . . . . . . . . 141

7.3 The upper limits are overlaid on the $\mathrm{P}_{1.4}-\mathrm{Y}_{S Z}$ and $\mathrm{P}_{1.4}-\mathrm{M}_{[500]}$ planes . . 146

7.4 X-ray and radio data of clusters RXCJ2129.8-5048 \& RXCJ0645.4-5413 147

7.5 The LLS- $\mathrm{L}_{X}$ plane for halo clusters . . . . . . . . . . . . . . . . . 149

8.1 High resolution image of the HT galaxy in A3771 . . . . . . . . . . 153

8.2 Fractional polarisation image of the HT galaxy . . . . . . . . . 155

8.3 The spectral index map and its error of the HT galaxy . . . . . . . . . 156

8.4 Rotation measure transfer function in our RM synthesis . . . . . . . 159

$8.5 \mathrm{~F}(\phi)$ spectrum in two pixels of the HT galaxy . . . . . . . . . . 161

8.6 RM map of the HT galaxy in A3771 . . . . . . . . . . . . . . . 162

8.7 Histogram of final RM values of the HT galaxy . . . . . . . . . . . . 164

8.8 Magnetic field orientation over the HT galaxy . . . . . . . . . . . . 165

8.9 Total intensity and polarisation profile along slices . . . . . . . . . . . 166

8.10 Total intensity and polarisation profile along slices . . . . . . . . . . . . 167

9.1 Comparison between current and future SZ cluster surveys . . . . . . . 172 


\section{List of Tables}

4.1 Details of the pre-CABB ATCA radio observations of our REXCESS sub-sample. 38

4.2 Details of the pre-CABB low-resolution images . . . . . . . . . . 38

4.3 Details of new CABB ATCA observations . . . . . . . . . . . . . . 39

4.4 Details of the low resolution $\mathrm{CABB}+$ pre-CABB images . . . . . . . . 39

4.5 Details of the high resolution images of the REXCESS sub-sample . . . 40

4.6 Details of the detected sources in REXCESS sub-sample . . . . . . . . 52

5.1 Flux of the embedded radio sources in the halo region . . . . . . . . . 83

5.2 Properies of the radio halo in A3888 . . . . . . . . . . . . . 87

5.3 Details of Stokes I and polarisation images of RXCJ0225.1-2928 . . . . 91

5.4 Features of the diffuse emission in RXCJ0225.1-2928 . . . . . . . . . . 97

6.1 Details of the redshift in a one degree radius around A3888 . . . . . . 107

6.2 Details of detected structures in the main population . . . . . . . . . . . 119

6.3 Details of the substructures of A3888 . . . . . . . . . . . . . 119

6.4 Details of detected structures in redshift range $0.0717-0.0856$ by DBSCAN . . . . . . . . . . . . . . . . . 121

7.1 Properties of the observed REXCESS sub-sample . . . . . . . . . . . 132

7.2 Upper limits of non-halo clusters . . . . . . . . . . . . . . . . . 137

7.3 Halo fraction in different radio halo surveys . . . . . . . . . . . . . 138

7.4 Slope of the best-fit of scaling relations . . . . . . . . . . . . . 144

8.1 Details of Stokes I and polarisation images of the HT galaxy . . . . . 154

8.2 RM Synthesis properties and detection capability over the CABB band . 160

A.1 Radio sources around the cluster RXCJ0006.0-3443 (C01) and their properties. . . . . . . . . . . . . . . . . . 197

A.2 Radio sources around the cluster RXCJ0049.4-2931 and their properties. 198

A.3 Radio sources around the cluster RXCJ0145.0-5300 and their properties. 199

A.4 Radio sources around the cluster RXCJ0211.4-4017 and their properties. 200

A.5 Radio sources around the cluster RXCJ0225.1-2928 and their properties. 201

A.6 Radio sources around the cluster RXCJ0345.7-4112 and their properties. 202

A.7 Radio sources around the cluster RXCJ0616.8-4748 and their properties. 203

A.8 Radio sources around the cluster RXCJ0645.4-5413 and their properties. 204

A.9 Radio sources around the cluster RXCJ1236.7-3354 and their properties. 205 
A.10 Radio sources around the cluster RXCJ2014.8-2430 and their properties. 206 A.11 Radio sources around the cluster RXCJ2023.0-2056 and their properties. 207 A.12 Radio sources around the cluster RXCJ2129.8-5048 and their properties. 208 A.13 Radio sources around the cluster RXCJ2149.1-3041 and their properties. 209 A.14 Radio sources around the cluster RXCJ2218.6-3853 and their properties. 210 A.15 Radio sources around the cluster RXCJ2234.5-3744 and their properties. 211

B.1 List of the published halos and this work (A3888) . . . . . . . . . . 213

B.1 List of the published halos and this work (A3888) . . . . . . . . . . . . 214

B.1 List of the published halos and this work (A3888) . . . . . . . . . . 215

B.1 List of the published halos and this work (A3888) . . . . . . . . . . 216

D.1 Details of the objects observed with the AAOmega. . . . . . . . . . . . . 221

D.1 Details of the objects observed with the AAOmega . . . . . . . . . . . . 222

D.1 Details of the objects observed with the AAOmega . . . . . . . . . . . . 223

D.1 Details of the objects observed with the AAOmega . . . . . . . . . . . 224

D.1 Details of the objects observed with the AAOmega . . . . . . . . . . . 225

D.1 Details of the objects observed with the AAOmega . . . . . . . . 226

D.1 Details of the objects observed with the AAOmega . . . . . . . . . . . 227

D.1 Details of the objects observed with the AAOmega . . . . . . . . . . . 228

D.1 Details of the objects observed with the AAOmega . . . . . . . . . . . . 229

D.1 Details of the objects observed with the AAOmega . . . . . . . . . . . 230

D.1 Details of the objects observed with the AAOmega . . . . . . . . . . . 231

D.1 Details of the objects observed with the AAOmega . . . . . . . . . . . 232

D.1 Details of the objects observed with the AAOmega . . . . . . . . . 233

D.1 Details of the objects observed with the AAOmega $\ldots \ldots . . \ldots 234$

D.1 Details of the objects observed with the AAOmega . . . . . . . . . 235

D.2 Table of the acronyms used in this thesis. . . . . . . . . . . . 238 


\section{$\S 1$. Introduction to Extragalactic Radio Sources in Galaxy Clusters}

In this chapter, a brief introduction to extragalactic radio sources found in galaxy clusters is given.

\subsection{Radio Galaxies}

It is believed that the majority of galaxy cores are the habitat of giant black holes. In these galactic interiors, the gravity of the black hole pulls the material towards the centre and generates an accretion disk. The accreting plasma rotates while it is falling towards the fast spinning black hole. The charged particles of the infalling plasma and the magnetic field of the black hole interact with each other and form a pair of collimated jets perpendicular to the accretion disk that push the material outwards. The resultant synchrotron emission associated with the expelled jets can be observed in radio wavelengths, and radio galaxies is the term used to describe radio-detected active galaxies.

Over the last few decades, observations of radio galaxies have indicated that radio sources exhibit different structures. Fanaroff and Riley (1974) divided radio galaxies into two distinct groups of morphology; Fanaroff and Riley Class I (FRI) and Fanaroff and Riley Class II (FRII). In FRI radio galaxies the radio luminosity decreases towards the outermost regions of the jets. Typically FRI sources are comprised of three components: the core, jets and the plumes.

In contrast, FRII radio sources are brightest at the ends of the jets that terminate in hot spots which reside in large lobes. Thus FRIIs host four components; core, jets, lobes and bright hot spots. In general, FRII sources are more luminous than FRI sources (Ledlow and Owen, 1996). It is believed that this luminosity difference is due to the different jet velocity. Jets in FRII sources have higher velocities resulting in more efficient energy transfer from the core, whereas FRI sources have lower jet velocities and less efficient energy transportations (Bicknell, 1995). Figure 1.1 shows an example of an FRI source. The core, jets and plumes are clearly visible. The jets and the plumes gradually lose their brightness. Here in this work we are mainly interested in asymmetric and distorted structures in radio galaxies which are found in galaxy clusters. A class of these complex sources have jets and lobes which are not entirely symmetric and not aligned with the core. These radio objects are called Bent-Tailed (BT) galaxies (Dehghan et al., 


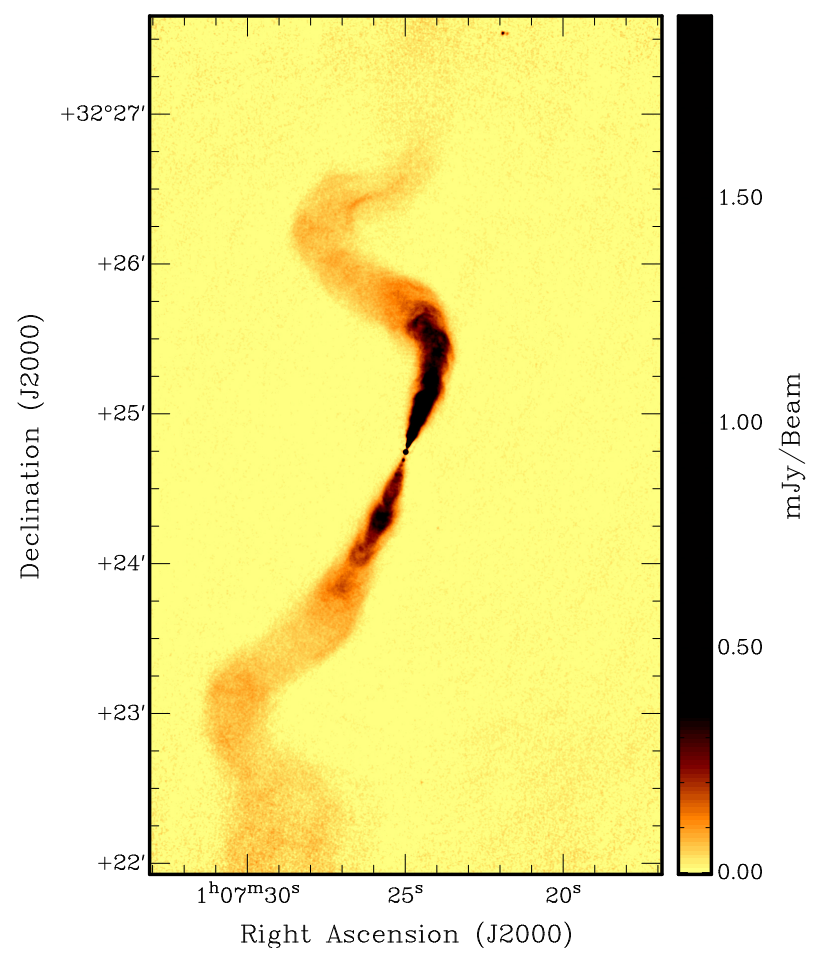

Figure 1.1: An example of an FRI radio galaxy (3C31). The core, jets and plumes components of the FRI radio galaxy are visible. The brightness gradually decreases from jets towards the plumes. The image is publicly available in fits format and could be found at heawww.harvard.edu/XJET (Hardcastle et al., 2002).

2014). BTs can exhibit more exotic radio structures; they might have double widely spaced bent jets known as "Wide-Angle-Tail" (WAT) galaxies or in contrast, they may have double narrowly spaced bent jets in the same direction as "Head-Tail" (HT) and "Narrow-Angle-Tail" (NAT) radio galaxies. The HT and NAT galaxies might have even more complex shapes where merely a single jet is observed due to projection effect and the radio structure resembles a "comet like" shape. In Figure 1.2, an example of an HT galaxy is shown. This HT galaxy has been discovered in this work and is presented in Chapter 8.

HT galaxies are used as powerful tools to probe the cluster environment. The majority of HT galaxies are located in over-dense regions in the local $(\mathrm{z}<0.1)$ Universe (Mao et al., 2009) with rich environments similar to galaxy clusters and galaxy groups. Furthermore, HTs have been shown to trace large scale structures and can be used in galaxy cluster detection (Smolčić et al., 2007; Blanton et al., 2004; Mao et al., 2010). In addition to detection of clusters, HTs have been used in a variety of ways to probe a diverse range of physical parameters in galaxy clusters; including density measurements of the intra-galactic medium (IGM, Freeland et al., 2008), detection of large scale shocks in the intra-cluster medium (ICM) (Pfrommer and Jones, 2011) and placing constraints on the cluster magnetic field over small scales (Guidetti et al., 2008; Pratley et al., 2013).

There are two mechanisms that have been suggested so far to explain the existence 


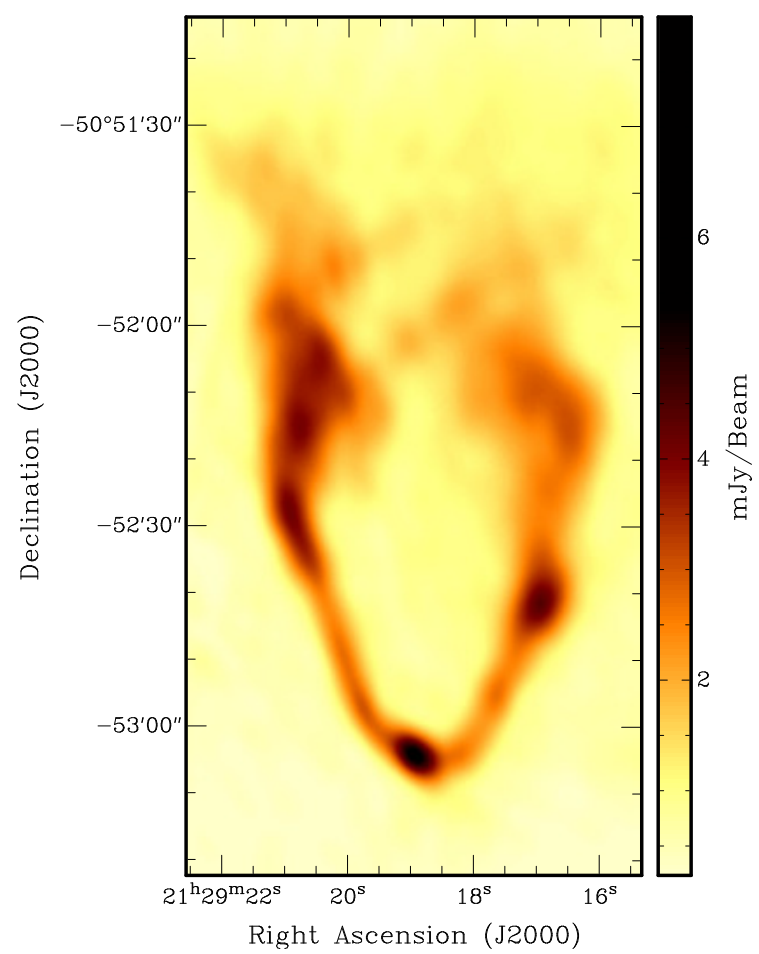

Figure 1.2: An example of a head-tail galaxy observed in the cluster Abell 3771. The core (head) and the bent jets (tails) of the HT galaxy are evident. This HT galaxy was observed at $1.4 \mathrm{GHz}$ with the Australia Telescope Compact Array (ATCA) in this work and is presented in detail in Chapter 8.

of tailed radio galaxies in galaxy clusters. In the first mechanism, it is believed that the curved jets in HT galaxies are associated with the motion of the host galaxy with relatively large peculiar velocity with respect to the ICM. In the rest-frame of the infalling galaxy, the interstellar gas experiences a wind which is the result of its rapid motion within the ICM hot gas (Miley et al., 1972; Begelman et al., 1979). This wind causes the galaxy's jets to move in the opposite direction to the galaxy's motion in the cluster. This effect is known as ram pressure bending of the radio jets (Gunn and Gott, 1972; Miley et al., 1972; Rudnick and Owen, 1976).

The second mechanism is known as "cluster weather" (Burns, 1998). Recent observations of galaxy clusters in different frequency domains (X-ray, optical and radio) in conjunction with N-body hydrodynamic simulations have indicated a highly complex environment in galaxy clusters. The ongoing accretion of mass and dark matter from inter-galactic filaments (Johnston-Hollitt et al., 2004a), bulk motion of the galaxies in the cluster and major cluster merging generate high velocity wind and extreme turbulence in the ICM. It is largely accepted that this long-lived so-called stormy weather can distort and displace the radio jets of the evolving radio galaxies in clusters (Burns et al., 2002). 


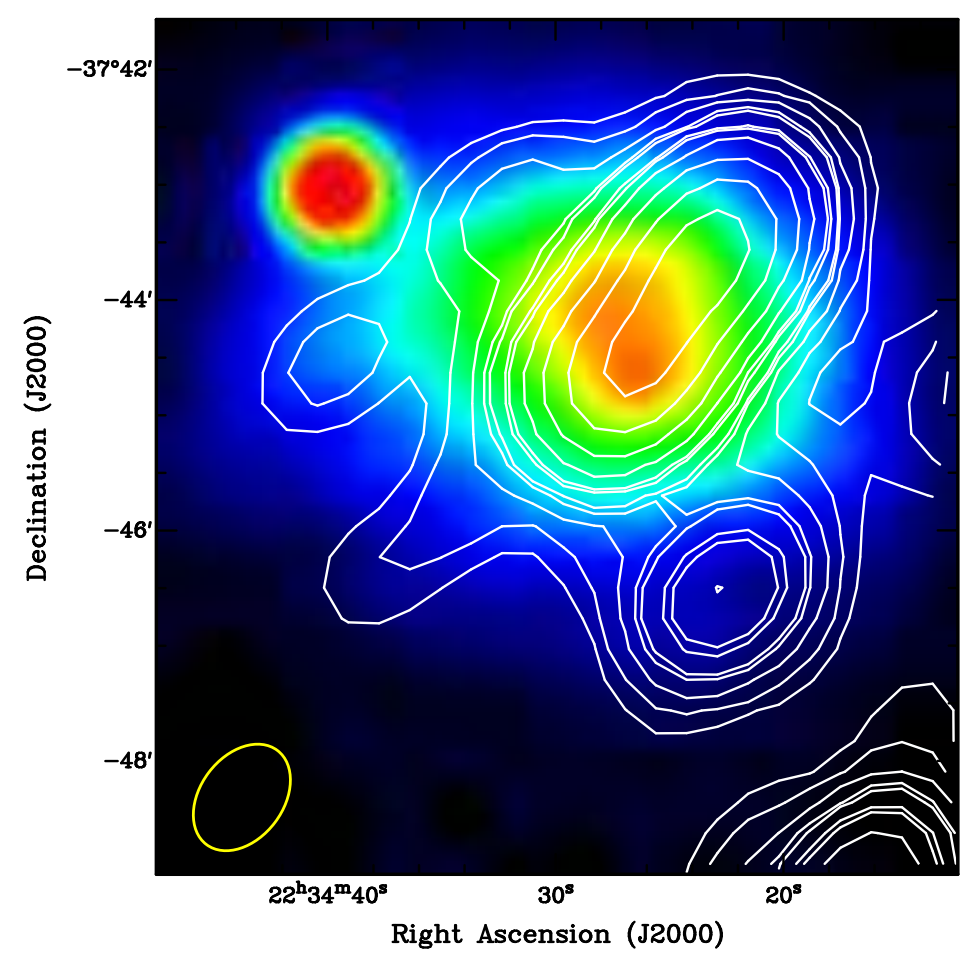

Figure 1.3: An example of a radio halo in A3888 presented in this work (see Chapter 5). The white contours show the halo emission in A3888 observed with the ATCA at $1.4 \mathrm{GHz}$. The contours start at $360 \mu \mathrm{Jy}_{\text {beam }}{ }^{-1}$ and are spaced in intervals increasing by a factor of 2 . The background is the X-ray image was observed with XMM-Newton (Pratt et al., 2009). The radio halo located inside the $\mathrm{X}$-ray emitting region of the cluster is evident.

\section{§1.2. Diffuse Radio Sources in Clusters}

\section{§1.2.1. Radio Halos}

With the advent of modern and considerably more sensitive radio telescopes, diffuse synchrotron emission has been observed in galaxy clusters. These spectacular radio sources are very hard to observe due to their very low surface brightness, particularly at the outermost regions where the brightness profile is steepest. There are two types of this kind of radio objects observed to date; giant radio halos and mini-halos.

Giant radio halos are cluster-scale radio emission in the central part of the clusters. The morphology of radio halos typically follows the distribution of gas in the ICM. The halo in A3888 (this work) is shown in Figure 1.3. The halo emission is inside the Xray emitting region of $\mathrm{A} 3888$. The first radio halo was observed in the Coma cluster (Willson, 1970) and subsequent follow-up observations across the radio spectrum with different telescopes have provided detailed spectral information on the object. Coma can be considered to host a typical radio halo with a steep spectral index $(\alpha \sim 1.2)$ that has radial steepening (Giovannini et al., 1993; Deiss, 1997). Figure 1.4 shows the steep spectrum of the radio halo in Coma. In the last decade, more deep observations 


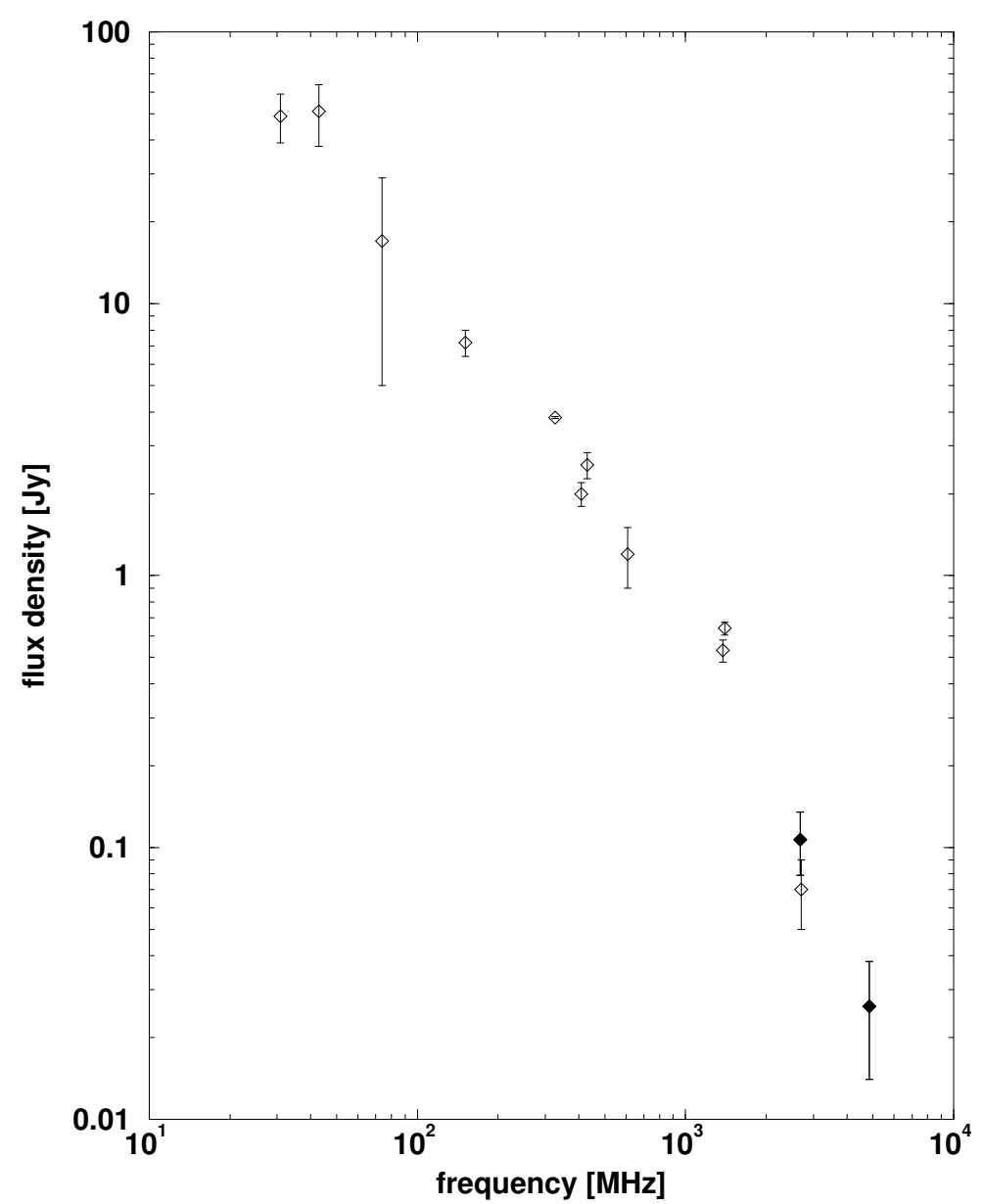

Figure 1.4: The multi-frequency radio spectrum of radio halo in Coma. The spectrum is quite steep which is typical in radio halos in clusters. The image is adapted from Thierbach et al. (2003).

of clusters have led to detection of around 62 radio halos to date (see B.1). It is now clear that all radio halos have steep spectra with an average spectral index value of $\alpha=$ $1.3^{1}$ (Ferrari et al., 2008; Feretti et al., 2012). Additionally, the spectral index maps of well-observed halos have shown that the spectral index has a value around 0.8 in the centre and radially decreases to the values around 1.8 (e.g. Giovannini et al., 1993, Feretti et al., 2004, Vacca et al., 2014, Shimwell et al., 2014). In the last decade, a few radio halos with very steep integrated spectral indices $(\alpha>1.5)$, known as ultra steep spectrum (USS) halos, have also been observed. Radio halos in clusters such as A697 (Kassim et al., 2001) with $\alpha=1.7$ to 1.8, A1914 (Bacchi et al., 2003) with $\alpha=$ 1.88, and MCSJ1149.5+2223 (Bonafede et al., 2012) with $\alpha \geq 2$ are the examples of USS halos. Although the number of detected USS radio halos are quite limited, due to the increasing number of low-frequency radio surveys such as the Multi-frequency Snapshot Sky Survey (MSSS) (Heald et al., 2015) with the Low-Frequency Array (LOFAR, van Haarlem et al., 2013) and the Galactic and Extragalactic All-sky MWA survey

\footnotetext{
${ }^{1}$ The convention of $\mathrm{s} \propto v^{-\alpha}$ is assumed.
} 
(Wayth et al., 2015) with the Murchison Widefield Array (MWA, Tingay et al., 2013), the number of USS radio halos is expected to grow.

Radio halos are mostly observed in clusters with high X-ray luminosity and have been detected over a wide range of redshifts. For instance, MACS J0417.5-1154 with $\mathrm{L}_{X}[0.1-2.4] \mathrm{keV} \simeq 2.5 \times 10^{45} \mathrm{erg} / \mathrm{s}$ is the halo cluster with the highest $\mathrm{X}$-ray luminosity (Dwarakanath et al., 2011) and PLCK G174.3-16.6 is the highest redshift halo cluster ( $\mathrm{z}=0.65$, van Weeren et al., 2014b, ) to date. MACS J0717.5+3745 (Dwarakanath et al., $2011)$ hosts the most powerful radio halo $\left(\mathrm{P}_{1.4 \mathrm{GHz}}=5 \times 10^{25} \mathrm{~W} / \mathrm{Hz}\right)$ whereas $\mathrm{A} 3562$ (Venturi et al., 2003) is the habitat of the least powerful radio halo $\left(\mathrm{P}_{1.4 \mathrm{GHz}}=2 \times 10^{23}\right.$ $\mathrm{W} / \mathrm{Hz}$ ) observed to date. Based on the information of the 62 known radio halos to date, we estimate that about $85 \%$ and $65 \%$ of the halos have a linear scale greater than 700 kpc and $1 \mathrm{Mpc}$, respectively. However, a few halos with smaller scales e.g. A2218 (Giovannini and Feretti, 2000), A3562 (Venturi et al., 2003) and A2294, (Giovannini et al., 2009) have also been observed.

The halo emission is often said to be unpolarised, however it is more correct to say that current observations limit the fractional polarised emission in halos to be less than 10\%. Govoni et al. (2013) carried out radio halo simulations that demonstrated that the radio halos should be polarised, though at present, the only two detected polarised radio halos are in A2255 (Govoni et al., 2005; Pizzo et al., 2009) and MACS J0717.5+3745 (Bonafede et al., 2009a). The lack of detected polarised emission in radio halos might be the result of insufficient sensitivity of the data, however, it is also known that mergerdriven turbulence can heavily distort the ordered magnetic field on scales less than observing beam and therefore cause an additional effect via beam-width depolarisation. In addition, within the magnetised ICM, there will be internal Faraday rotation which can significantly depolarise the halo emission. The internal Faraday depolarisation arises due to the fact that the emission of each electron travels through the magnetised plasma with different lengths along the line-of-sight, hence experiences different Faraday rotation. This can result in significant variation of the superimposed Faraday rotation across the source even if the magnetic field of the foreground medium and the electron density is homogeneous and isotropic. In Chapter 2, radio halo generation models and their implications are discussed in greater detail.

\section{\$1.2.2. Radio Relics}

Radio relics are another class of large-scale diffuse radio emissions in clusters. Radio relics are only found on the outskirts of clusters and have been observed with different sizes and at various cluster-centric radii.

Radio relics have less regular morphologies and can be elongated arcs or irregular in shape, however, more symmetric and roundish morphologies have also been observed 


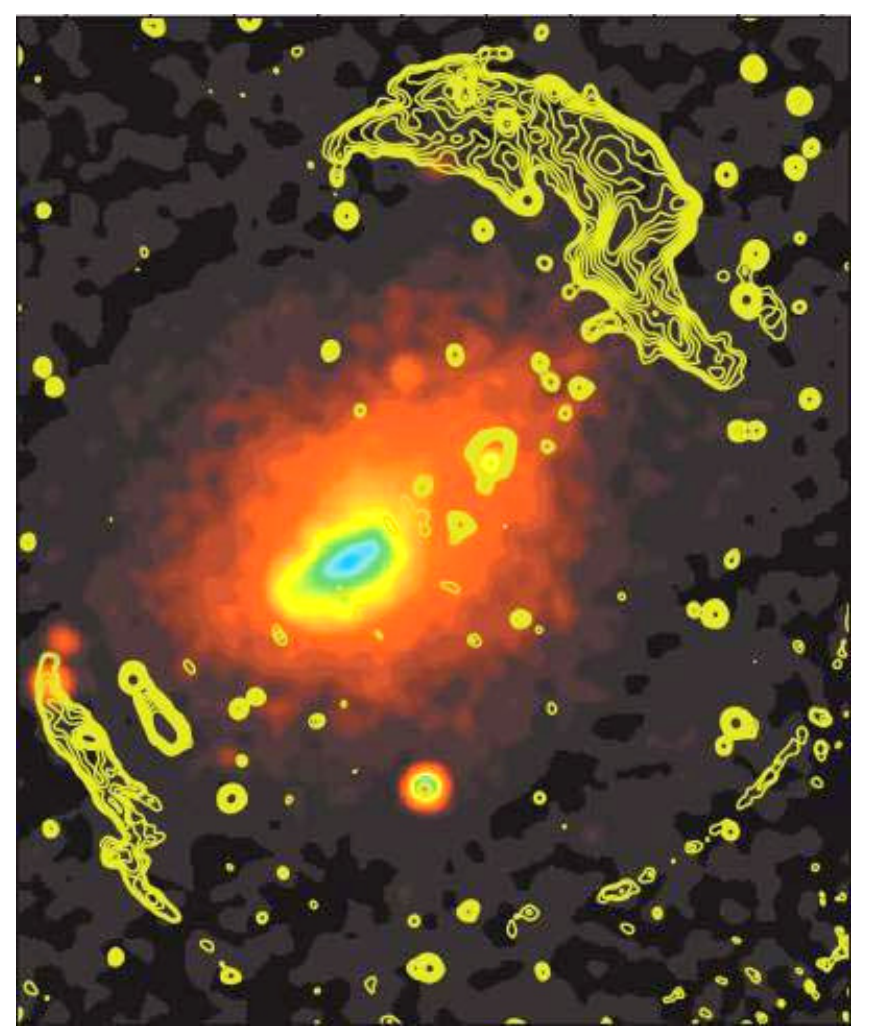

Figure 1.5: A double radio relic detected in Abell 3667 (Johnston-Hollitt et al., 2002; JohnstonHollitt, 2003). The yellow contours show the radio emission observed with the ATCA at 1.4 GHz. The background image is the ROSAT X-ray image. Both of the relics are located at the edge of the X-ray emitting region of the cluster.

(e.g. A2443, Cohen and Clarke, 2011). The double radio relics in A3667 (see Figure 1.5, Johnston-Hollitt et al., 2002, Johnston-Hollitt, 2003) and a very narrow radio relic in CIZA J2242.8+5301 (Harris et al., 1993) also known as the "sausage relic" (van Weeren et al., 2010) are spectacular examples of elongated relics in clusters. Radio relics have total steep spectral indices varying from 1 to 1.6 in elongated relics such as A3667 (Johnston-Hollitt et al., 2002; Johnston-Hollitt, 2003) and PLCKG287.0+32.9 (Bonafede et al., 2014) and from 1.1 to 2.9 in more regular relics such as A2256 (Kale and Dwarakanath, 2010; Owen et al., 2014) and ZwCl 0008.8+5215 (van Weeren et al., 2011b). The steep spectral index of relics can be explained by first order Fermi acceleration. Radio relics are highly polarised (up to $40 \%$ at $1.4 \mathrm{GHz}$ ) and are commonly used to infer the magnetic field of clusters (Johnston-Hollitt, 2004; Clarke and Ensslin, 2006a; Bonafede et al., 2009b).

In some clusters such as A2255 (Burns et al., 1995), A2256 (Bridle and Fomalont, 1976) and A1300 (Reid et al., 1999), both a radio halo and relic are present.

All detected radio relics are found in merger clusters and interestingly, are commonly located at the region where bow-shocks are present. Thus, it is believed that merger driven shocks trigger radio relic formation in clusters. More recent simulations suggest that the combination of accretion shocks and merger shocks are behind the ori- 
gin of the radio relics (Ensslin et al., 1998; Enßlin and Gopal-Krishna, 2001).

Future radio observations of clusters with more sensitive telescopes are expected to find more radio relics in clusters and therefore more precise calculations of the ICM electron density and magnetic field at the peripheral regions of clusters will be achievable. We have not discussed the radio relic generation models in great detail because relics are not of special interest in this work.

\subsubsection{Mini Halos}

Radio observation of some clusters revealed that there is another class of diffuse radio halos in clusters; mini-halos which are less extended than giant radio halos. Mini-halos are found at the centre of the clusters but their largest linear scale does not exceed 500 kpc. Diffuse extended emission in the Perseus cluster (Sijbring, 1993) and more recent discoveries in the Phoenix cluster (van Weeren et al., 2014a) and RXCJ1720.1+2638 (Giacintucci et al., 2014) are the examples of mini-halos.

Like radio halos and relics, mini-halos have low surface brightness and steep spectra. However, in contrast with giant radio halos, mini halos have only been found in relaxed clusters which might suggest that opposed to radio halos, mini-halos do not arise due to the major merging activity in the cluster. Due to different ICM properties in relaxed clusters, mini-halos have not been considered as the scaled version of radio halos and they were mostly treated as a separate class of diffuse emission sources in clusters. However, in more recent works, the connection between mini-halos and giant radio halos has been revised. For instance, Brunetti and Jones (2014) put forward the possibility of an evolutionary transition from mini halo to giant radio halo under some circumstances. Soon after, Bravi et al. (2015) investigated the possibility of a correlation between mini-halo occurrence and the turbulence in clusters. Although several attempts have been made to address the mini-halo occurrence in clusters, due to the small number of detected mini-halos to date (20 mini-halos, van Weeren et al., 2014a and the references therein), the generation and evolution of mini-halos are not completely understood.

Although we discuss mini-halos here for completeness, investigation of mini halos is not the main interest in this work. General properties of galaxy clusters and the physical mechanisms for non-thermal emission in clusters are discussed in the next chapter. 


\section{§2. Radio Halos: Theoretical Overview}

Our main goal in this work was to shed more light on the radio halo statistics. To achieve this, we carried out a radio halo survey and utilised the radio images of 15 clusters which were observed with Australia Telescope Compact Array (ATCA) by Assoc Prof Melanie Johnston-Hollitt to discover possible radio halos. Moreover, we have supplemented our work with radio halos from the literature to perform statistical analysis of halos scaling relations and test the thermal/non-thermal properties of the clusters with/without radio halos. For the purpose of this study, this chapter first provides a clear theoretical background to the energy loss and acceleration mechanisms of cosmic ray electrons, which are vital in radio halo generation in clusters with magnetic field strength of a few $\mu \mathrm{G}$. We later discuss the two competing radio halo generation mechanisms, compare their predictions and verify their validity with respect to current halo observations.

\section{$\S 2.1$. Galaxy Clusters}

In the bottom-up structure formation scenario in modern cosmology, dark matter (DM) is the major component of galaxy clusters and DM halos are the first structures to form. The luminous matter is formed in later stages where the baryons are trapped and condensed in the gravitational potential well of the dark matter halo. In this process, hundreds, even thousands of galaxies and diffuse hot gas fall into the gravitation potential well of the DM halo. The cluster gradually grows until the mass of the system increases to the order of $10^{14}-10^{15} \mathrm{M}_{\odot}$ and the size extends beyond one Mpc (the size could be extended to four $\mathrm{Mpc}$ ). As the inter-galactic gas is pulled towards the centre of the cluster, the gas density increases to $10^{-1}-10^{-4}$ atoms $\mathrm{cm}^{-3}$ and the hot plasma gradually sinks in the central part of the potential well to form the intra-cluster medium (ICM). The temperature of the ICM gradually increases up to $10^{8}$ Kelvin and the thermal electrons in the ICM emit X-ray emission via thermal bremsstrahlung ${ }^{1}$.

In summary, a galaxy cluster mainly contains DM, which encompasses to 70-80\% of the total mass. The remainder is baryonic matter in the form of galaxies and hot gas. The galaxies only take up few percent and the ICM is about $10-20 \%$ of the entire content.

The other important component of galaxy clusters are relativistic particles known as cosmic rays (CRs). Cosmic ray sources in the clusters are believed to be supernova rem-

\footnotetext{
${ }^{1}$ Thermal bremsstrahlung is an electromagnetic radiation produced via deceleration of an electron due to passing another charged particle such as atomic nucleus.
} 
nants, star-forming galaxies, active galaxies (AGNs), galactic winds and shock waves that were generated during large-scale structure formation. Cosmic rays are known to be the major source of diffuse radio and gamma ray emission in galaxy clusters (Brunetti and Jones, 2014).

Galaxy clusters can be observed by gamma ray, X-ray, optical and radio telescopes. Each of these observations provide invaluable and often complementary information about the cluster properties and evolution. While the optical observations could reveal the dynamics, distance and velocity dispersion of the cluster, X-ray observation of the clusters carry information about the mass, temperature of the ICM, size and the electron density in the ICM. In addition, the X-ray data can be combined with the optical data to provide a clear insight into the dynamical status of the cluster. Radio observations, on the other hand, are ideal to probe the magnetic field and the energy content of the relativistic particles in the clusters. Throughout this work, we have combined radio, optical and X-ray observations of the clusters under study to accurately determine their properties.

\section{$\S$ 2.1.1. Cluster Morphology}

Since radio halos have only been observed in merging clusters, understanding the morphology of the clusters are of particular importance in radio halo studies. This is because it is believed that lack of dynamical interaction in clusters can switch off the halo generation, thus, merging is a key ingredient in radio halo generation.

The merging of galaxy clusters is the most energetic event to happen in the Universe following the Big Bang. This event produces powerful shocks and turbulence within the ICM, where merger-driven shocks dramatically heat the ICM and raise the energy density of the electrons and protons in the plasma.

From the cluster's dynamics point of view, all galaxy clusters can be sub-divided into two groups; merger (disturbed) and non-merger (relaxed). The dynamical activity of the clusters has significant imprints on their general properties. The cluster global properties such as mass, velocity dispersion, temperature and galaxy distribution are heavily modified during the dynamical interactions in clusters. Cluster merging also alters the magnetic field and the ICM temperature profile via generated shocks and turbulence. As mentioned earlier, the combination of optical and X-ray observations of clusters reveal the dynamical activity of the clusters. This signifies that radio halo studies are not purely dependent on radio data of the clusters, and often complementary $\mathrm{X}$-ray and optical data are vital for halo studies. 


\section{§2.2. Magnetic Field in Galaxy Clusters}

Interestingly, observation of radio halos in clusters (e.g. Coma) is one of the most solid pieces of evidence found in favour of the existence of a cluster-wide magnetic field in clusters. A comprehensive understanding of the magnetic field of galaxy clusters is especially important to answer questions about the generation of radio halos in clusters.

Radio halos are used to estimate the lower-limit of the cluster magnetic field by assuming equipartition of the energy of the CR population and the magnetic field (Tribble, 1991; Beck and Krause, 2005). In this method, the total energy of the cosmic ray electrons (CRes), cosmic ray protons (CRps) and magnetic field are assumed to be a minimum to estimate a lower limit of the magnetic field within the cluster. Using this method has yielded $\mathrm{B}_{c l} \sim 0.1 \mu \mathrm{G}$ (Gursky and Schwartz, 1977; Rephaeli and Gruber, 1988). The derived magnetic field from radio halos in the equipartition method is strongly dependent on the CR profile and energy density models which describe the electron population. Therefore, the inferred magnetic field might be inconsistent if different models of the CR profile are assumed.

In addition, calculations of the Faraday Rotation Measure (RM) of the sources inside or in the background of clusters can be used to infer the magnetic field of the cluster (Hennessy et al., 1989; Kim, 1990; Clarke et al., 2001; Johnston-Hollitt, 2003; JohnstonHollitt and Ekers, 2004; Clarke, 2004). The RM technique has a superior advantage over the equipartition method as CR assumptions are not required.

Further investigation of more sensitive polarised maps of the clusters has led to more developments in study of the magnetic field of the clusters. Brunetti et al. (2001) and Bonafede et al. (2010) studied Coma in great detail to determine the magnetic field profile of the cluster. Soon after, Bonafede et al. (2011) used the fractional polarisation of the sources in the clusters to place firmer constraints on the power spectrum of the magnetic field.

The majority of the investigations of the cluster magnetic fields have yielded the strength of the magnetic field to be around $1-5 \mu \mathrm{G}$ in the central part. These values are consistent with the multiple MHD simulations of the galaxy clusters (Dolag et al., 1999, 2002; Dolag and Stasyszyn, 2009; Mikellides et al., 2011). The combination of current observations and simulations suggests that the cluster magnetic field follows the ICM density profile of the cluster (Dolag et al., 2005). In the following, the Faraday rotation theorem and observations are discussed.

\section{$\S 2.2 .1$. Faraday Rotation}

Linearly polarised light is composed of two opposite-handed circularly polarised components. As the polarised electromagnetic wave travels through a magnetised medium 
the phase angle of the opposite-handed components change differently if the magnetic field has a component along the direction of the wave propagation. This is basically a natural result due to a difference in refractive indices in the magnetised plasma. The lefthand component travels slightly slower than the right hand component, thus, the plane of the polarised wave is rotated. The phase angle rotation is proportional to the magnetic field across the medium and the electron density in the plasma and the wavelength of the wave. This rotation rate is termed as rotation measure and is given by:

$$
\mathrm{RM}=\frac{e^{3}}{2 \pi m_{e}^{2} c^{4}} \int_{0}^{L} n_{e}(l) B_{\|}(l) d l,
$$

where $e$ and $m_{e}$ are the electron charge and mass, respectively. The other parameters are $c$, the speed of light, $n_{e}$, the electron density, $B_{\|}$, the line-of-sight component of the magnetic field and $l$, the path length along our line of sight where the rotation occurs. In practical units the RM can be written as:

$$
\mathrm{RM}\left[\frac{\mathrm{rad}}{\mathrm{m}^{2}}\right]=812 \int_{0}^{L} n_{e}\left[\mathrm{~cm}^{-3}\right] B_{||}[\mu \mathrm{G}] d l[\mathrm{kpc}]
$$

A positive RM value indicates that the average magnetic field is directed toward us and a negative RM value is indicative of an average magnetic field directed away from us. The observed polarisation position angle is given by:

$$
\psi_{O b s}=\psi_{I n i}+\lambda^{2} R M
$$

where $\psi_{\text {Ini }}$ is the initial position angle before rotation. Accurate measurement of the $\mathrm{RM}$ is subject to observation of the radio sources at multiple frequencies to effectively overcome the $n \pi$ ambiguity $^{2}$ problem. All the measured RM values of extra galactic sources in or behind galaxy clusters consist of at least three components: the RM of the source itself; the Galactic RM contribution; and the RM due to the ICM of the cluster. The RM of the Galactic plane has been estimated (Johnston-Hollitt et al., 2004b; Oppermann et al., 2012, 2015) and should be subtracted from the observed RM. In recent years, the desire for more accurate RM calculation has led to a new development known as "RM synthesis" (Brentjens and de Bruyn, 2005); which uses broad-band polarisation observations to infer more accurate RM values. However, the effectiveness of this method is strongly dependent on the bandwidth and channel width of the radio data. This method is also more advanced in terms of having the capability of disentangling the fore/background RM screens along the line of sight of the source. We have used this

\footnotetext{
${ }^{2}$ The $n \pi$ ambiguity is raised as there is no observational difference between $\psi$ and $\psi+n \pi$. Therefore the observations have to be very close in frequency space and an assumption must be made that there is no additional rotation between the adjacent frequency points.
} 


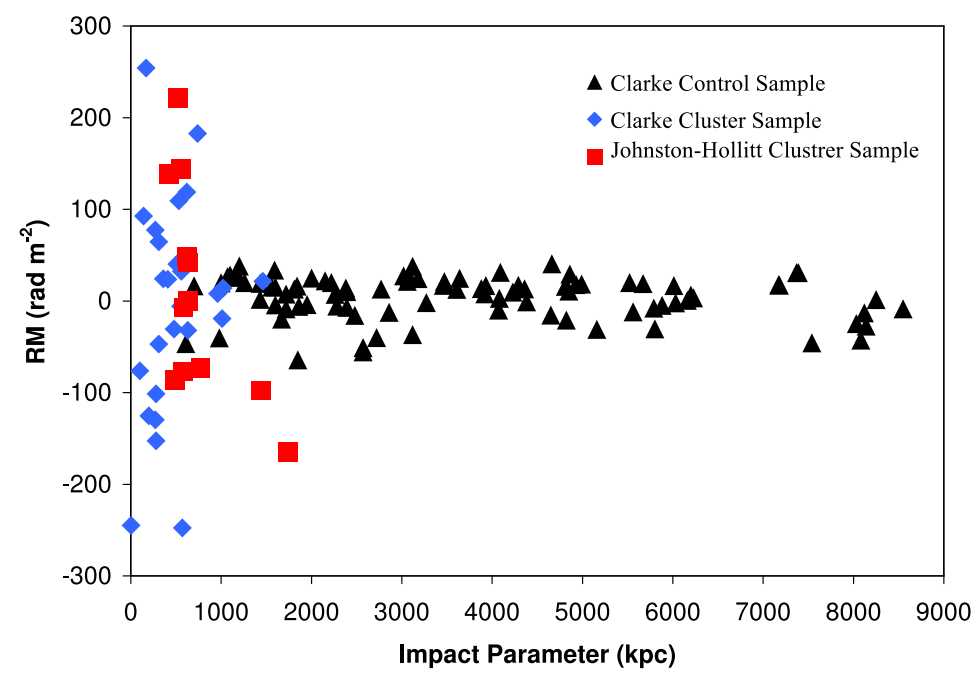

Figure 2.1: The distribution of measured RM values derived by Clarke et al. (2001) and JohnstonHollitt and Ekers (2004) vs the impact parameter for a sample of galaxy clusters. The image is adapted from Johnston-Hollitt and Ekers, 2004.

method to determine the magnetic field direction of a radio galaxy. The results of this analysis are presented in Chapter 8.

\section{$\S$ 2.2.2. RM Observations of Clusters}

Early RM investigations of clusters date back to the 1980's, where Vallee et al. (1986) and Hennessy et al. (1989) found an excess RM in a sample of Abell clusters. Kim (1990) also found excess RM in Coma cluster. Later on, Kim et al. (1991) and Feretti et al. (1999) made use of the RM maps of the radio sources in Coma and A119 to pinpoint the existence of $\mu \mathrm{G}$ strength magnetic field in those clusters. Clarke et al. (2001); Johnston-Hollitt (2003); Johnston-Hollitt and Ekers (2004); and Clarke (2004) investigated the rotation measure of polarised sources inside or in the background of a sample of clusters, and reported excess RM compared to their control sample, which were non-cluster polarised sources located at radii beyond the X-ray emitting region of the cluster. Their statistical analyses were more accurate than the first work presented by Kim et al. (1991). The distribution of RM values (Clarke et al., 2001; Johnston-Hollitt and Ekers, 2004) vs impact parameter of a statistical sample of clusters are shown in Figure 2.1. The excess RM of the sources in or behind the clusters in contrast with the control sample is evident. It is thought that the excess RM is caused by the line of sight component of the cluster magnetic field.

The quest for a global model to describe the cluster magnetic field still persists in radio astronomy. With the advent of more sensitive radio telescopes with greater bandwidth and enhanced polarimetry, more accurate RM studies will become available 
to investigate the magnetic field of the clusters (Johnston-Hollitt et al., 2015). Apart from the fact that the magnetic field in clusters is a vital ingredient in halo formation, our improved understanding of the magnetic field profile can provide a clearer picture of the diffusion of the CRes on Mpc-scales in clusters. Since CRes propagate with different velocities in various magnetic field strengths, determining the magnetic field values and its profile are helpful to explain different sizes of the observed halos. It can also be useful to describe current radio halos brightness profiles.

\section{$\S 2.3$. Non-thermal Emission and Losses in Galaxy Clus- ters}

As mentioned earlier, cosmic ray electrons (primary electrons) are injected by the cosmic ray sources (see $\S 2.1$ ) into the ICM. The observations of diffuse non-thermal radio emission in clusters are the most direct evidence in favour of existence of high energy electrons $(\gamma>1000)$. It is known that CRes gain and lose energy in several thermal and non-thermal interactions in clusters. Therefore, understanding the evolution of the energy budget of the relativistic particles and particularly the CRe population can provide a clear insight into the generation of Mpc-scale non-thermal radio emissions in clusters. The combination of various non-thermal energy loss mechanisms such as synchrotron, inverse Compton and Coulomb effectively modify the energy loss rate of the electrons in clusters that can explain the observed steep spectrum of diffuse radio emission in clusters. In the following subsections a brief introduction to electron non-thermal emissions and losses are given.

\section{§2.3.1. Synchrotron Losses}

Radio emission from AGNs and the ICM are caused by synchrotron radiation. Synchrotron radiation is based on the motion of the relativistic electrons in a helical curve along magnetic field lines. This emission is directly dependent upon the energy of the electron population, $\gamma$, and the magnetic field intensity in the cluster where $\gamma$ is the Lorentz factor. Cosmic-ray electrons with energies in the range $10^{9}$ to $10^{14} \mathrm{eV}$ have $\gamma$ of order of $10^{3}$ to $10^{8}$ and such cosmic-ray electrons are called ultra relativistic. These electrons still move on spiral paths along magnetic field lines, but the angular frequencies of their orbits are lower because the inertial masses of the electrons are higher by a factor of $\gamma$ :

$$
\omega_{B}=\frac{e B}{\gamma m_{e} c}=\frac{\omega_{G}}{\gamma}
$$


where $B$ is the magnetic field, e is the electron charge. The time-averaged synchrotron radiation power for a single electron for a given pitch angle $\alpha$ is given by:

$$
P=\frac{2 e^{2}}{3 c^{3}} \gamma^{2} \frac{e^{2} B^{2}}{m^{2} c^{2}} v^{2} \sin ^{2}(\alpha)
$$

if we replace $\mathrm{B}^{2}$ by the magnetic energy density, $\mathrm{U}_{B}=\frac{B^{2}}{8}$ and take the Thomson cross section, $\sigma_{T}=\frac{8 \pi}{3}\left(\frac{e^{2}}{m_{e} c^{2}}\right)^{2}$, into account, the power can be expressed as:

$$
P=2 \sigma_{T} \beta^{2} \gamma^{2} c U_{B} \sin ^{2}(\alpha)
$$

where $\beta=\mathrm{v} / \mathrm{c}$. The average synchrotron power for an electron population is simply an integration over the pitch angles:

$$
P=\frac{4}{3} \sigma_{T} \beta^{2} \gamma^{2} c U_{B}
$$

The observed frequency of the synchrotron radiation is given by:

$$
v_{\mathrm{syn}}=\frac{3 e B}{2 \pi m_{e} c} \gamma^{2} \simeq 1 \mathrm{GHz} \frac{B}{\mu \mathrm{G}}\left(\frac{\gamma}{10^{4}}\right)^{2},
$$

The energy density of an electron population is well described by a power-law with the index, $\delta$, while the plasma is optically thin and hence opaque to synchrotron radiation. The monochromatic radiation spectrum can be explained with a power-law with a spectral index $\alpha=(\delta-1) / 2$. The typical spectral indices for radio sources are between 0.7 to 0.8 . However Mpc-scale diffuse radio sources (halos, relics and minihalos) typically have steeper spectral indices.

The loss function $b(\gamma)$ for synchrotron emission is given by (Sarazin, 1999):

$$
b_{\mathrm{syn}}(\gamma)=\frac{4}{3} \frac{\sigma_{T}}{m_{e} c} \gamma^{2} U_{\mathrm{B}}=1.30 \times 10^{-21} \gamma^{2}\left(\frac{B}{1 \mu \mathrm{G}}\right)^{2} \mathrm{~s}^{-1} \text {, }
$$

where $U_{B}=B^{2} /(8 \pi)$ is the magnetic field energy density and $\sigma_{T}$ is the Thomson crosssection

\section{§2.3.2. Inverse Compton Losses}

Inverse Compton losses occur when high energy electrons $\left(\varepsilon_{e}=\gamma m_{e} c^{2} \gg m_{e} c^{2}\right)$ lose energy by scattering off a low energy photon $\left(\varepsilon_{p h}=h v \ll m_{e} c^{2}\right)$. This phenomenon is exactly the inverse process of Compton scattering effect in which low energy electrons gain energy from the high energy photons. The majority of the inverse Compton losses in galaxy clusters are associated with the synchrotron emitting relativistic elec- 
trons which upscatter the cosmic microwave background (CMB) photons. The energised blue-shifted CMB photons are then observed in the hard X-ray regime at $v \sim 2 \times$ $10^{8}-5.3 \times 10^{9} \mathrm{GHz}$ which corresponds to energy range $0.9-22 \mathrm{keV}$. The electron population undergoing IC have a the same origin as synchrotron emitting electrons, therefore have power-law energy distribution and similar spectral index. In the situation that the energy density of the CMB photons becomes greater than the synchrotron energy density $\left(\mathrm{U}_{I C}<\mathrm{Usyn}\right)$ the synchrotron emission is heavily suppressed due to extreme IC loss. This process is called the IC (radio) catastrophe. However, this phenomenon is expected to happen only in high redshift $(\mathrm{z} \sim 1)$ clusters with $\mu \mathrm{G}$ magnetic fields as the CMB photon energy density increases at higher redshifts.

The formula of the IC loss due to scattering off the CMB photons is very similar to the synchrotron loss expression. The only difference is the magnetic energy density which should be replaced by the CMB photons energy density. The loss function of IC from the CMB photons is given by (Sarazin, 1999):

$$
b_{\mathrm{IC}}(\gamma)=\frac{4}{3} \frac{\sigma_{T}}{m_{e} c} \gamma^{2} U_{\mathrm{CMB}}=1.37 \times 10^{-20} \gamma^{2}(1+z)^{4} \mathrm{~s}^{-1} .
$$

$U_{C M B}$ is the energy density of the CMB photons at $z$, the redshift of the cluster. The expressions of the synchrotron/IC losses are only valid when $\gamma \gg 1$.

\section{§2.3.3. Coulomb Losses}

Relativistic electrons lose energy while travelling in an ionised plasma due to Coulomb collisions with heavy ions. The Coulomb loss is more dominant when the plasma mostly contains low energy electrons $\gamma \lesssim 200$, whereas the synchrotron and IC losses are more efficient when the electron population has higher energy $\gamma \gtrsim 200$ (Sarazin, 1999). The Coulomb loss is given by (Sarazin, 1999):

$$
b_{\text {Coul }}(\gamma) \approx 1.2 \times 10^{-12} n_{e}\left[1.0+\frac{\ln \left(\gamma / n_{e}\right)}{75}\right] \mathrm{s}^{-1} .
$$

where $\mathrm{n}_{e}$ is the thermal electron density in the cluster.

\section{§2.3.4. CRe Life-time}

The time-scale of the energy loss or the synchrotron radiation life-time of electrons can be directly derived if the Lorentz factor and the loss function are known. The loss time is given by:

$$
t_{\mathrm{loss}}=\frac{\gamma}{b(\gamma)} .
$$

As shown in Figure 2.2, electrons with an energy range of $100-500 \mathrm{MeV}$ have the 


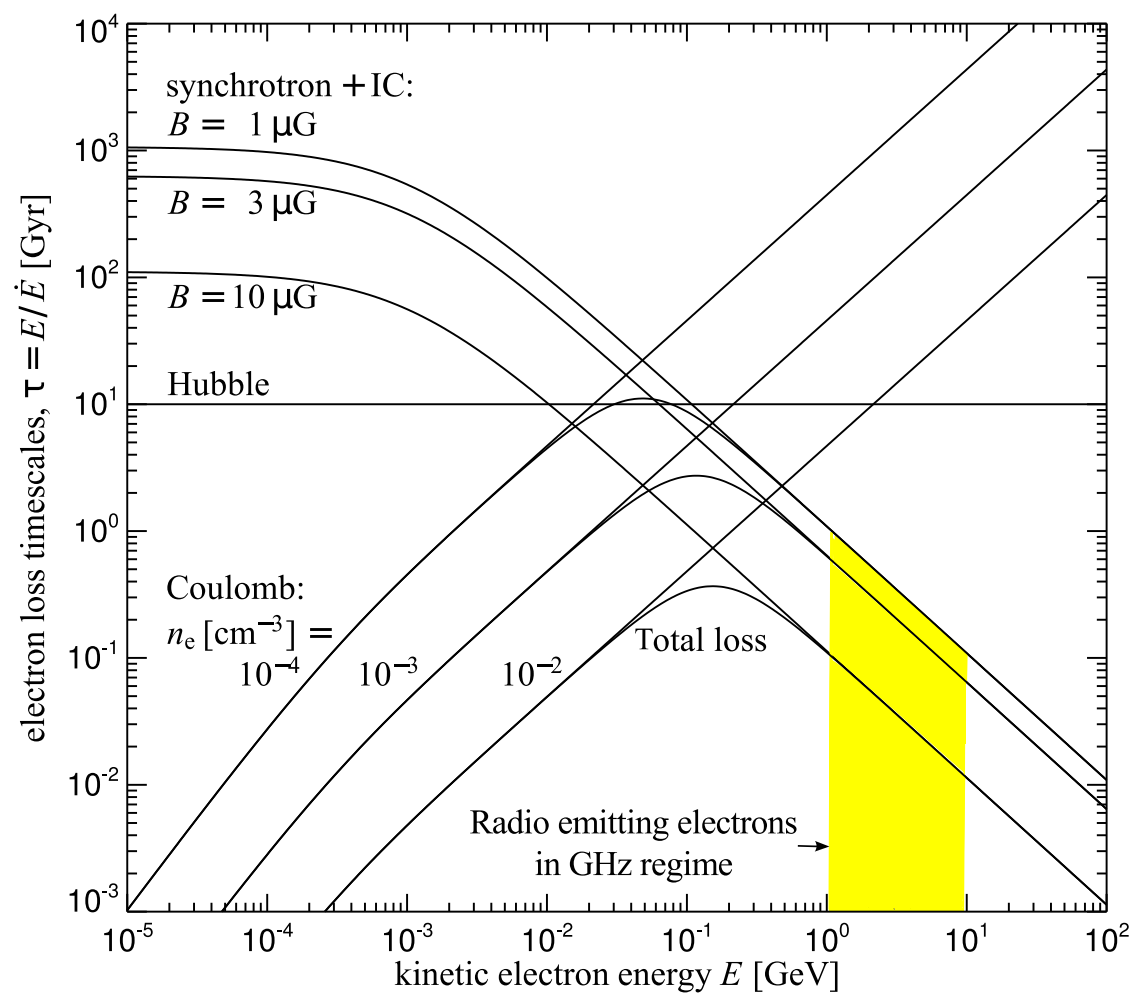

Figure 2.2: CRe life-time for various magnetic fields and densities that range from the core to the outskirts in galaxy clusters. Coulomb, IC and synchrotron loss processes are also shown. The yellow colour shows the energy range of the $\mathrm{GHz}$ radio emitting electrons in clusters. According to the plot, electrons have the synchrotron life-time between 0.1-1 Gyr in the magnetic field strength of $3 \mu \mathrm{G}$ and electron density of $10^{-3}\left[\mathrm{~cm}^{-3}\right]$. This plot is adapted from Ensslin et al., 2011.

longest life-time of about $10^{10} \mathrm{yr}$ in clusters with magnetic fields of a few of $\mu \mathrm{G}$. Since this time-scale is comparable to the cluster life-time, it is expected that a large reservoir of mildly relativistic electrons exists in clusters. However, with the increase of the IC loss at higher redshift $(\mathrm{z} \simeq 1)$ due to the rise of $\mathrm{CMB}$ photon number density in clusters, the accumulation of mildly energetic electrons is halted in high redshift clusters (Sarazin, 1999). Figure 2.2 also indicates that the electrons with $\gamma \approx 5000$ have a lifetime of less than $10^{8} \mathrm{yr}$. It is believed that electrons with such energies are associated with the Mpc-scale radio emission in clusters. In the next section, a brief introduction to high energetic non-thermal emission in galaxy clusters is given.

\section{§2.4. Secondary Cosmic Ray Electrons in Clusters}

In a galaxy cluster, in addition to primary electrons, which are the pre-existing electrons produced by CR sources, there is another electron population known as secondary electrons that have a different production mechanism. Relativistic secondary electrons are the product of charged pions decay in the interactions between the CRps and the hadrons of the ICM in clusters (Vestrand, 1980). Secondary CRes are thought to be vital in one 
of the halo generation models. All the cosmic ray production sites mentioned earlier (see $\S 2.1$ ) are also the sources of CRp injection into galaxy clusters. Since the energy loss rate of the CRps is much less than the CRes, they are much longer lived. Therefore, it is thought that a large content of highly energised long-lived CRps accumulates in clusters. The abundance of CRps in galaxy clusters is sufficiently significant to produce a considerable amount of secondary CRes. The observed radio halos in the $\mathrm{GHz}$ regime should have an electron population with energy of about $10 \mathrm{GeV}$ in the presence of $\mu \mathrm{G}$ magnetic fields. An electron population with such energy has a maximum life-time of $0.1 \mathrm{Gyr}$. In the case that a radio halo is formed from secondary CRes, the parent CRps should have energies of about $100 \mathrm{GeV}$. The CRps with such energies have a life-time of the order of the Hubble time. This suggests that the secondary electrons are ideal ingredients for the halo formation in galaxy clusters. However, the observational implications of radio halos formed by secondary CRes are not entirely supported by current halo observations. The halo generation from secondary electrons is discussed in more details in $\S 2.7$.

\section{§2.5. Gamma Ray Emission in Clusters}

There are currently two competing radio halo generation models, that are widely accepted by the radio astronomy community. These two models are re-acceleration model and hadronic model. Diffuse gamma ray emission in clusters is a unique testing tool to distinguish between these generation models. In addition, gamma ray observations of clusters are used to confine the energy density of the CRp. Another important byproduct of hadronic interactions between the CRps and the ICM protons is gamma rays that are generated via neutral pion decay $\left(\pi^{0} \rightarrow 2 \gamma\right)$. DM annihilation and IC emission due to upscattering of ultra-relativistic electrons $\left(\gamma>10^{5}\right)$ from the CMB photons are also expected to produce gamma rays in clusters (Petrosian et al., 2008). However, the contribution of the latter is expected to be sub-dominant (Vazza et al., 2014). The quest for diffuse gamma ray emission in clusters has been broaden by using gamma ray satellites such as the Fermi Large Area Telescope (Fermi-LAT) and the ground-based Major Atmospheric Gamma-ray Imaging Cherenkov Telescopes (MAGIC). Despite numerous gamma ray observations in the wide energy range of $20 \mathrm{MeV}$ to $300 \mathrm{GeV}$, detection of diffuse gamma ray emission in clusters has to date been controversial or insignificant. For instance, Abdo et al. (2009) carried out very deep ( $78 \mathrm{hrs}$ ) high energy observations $(>100 \mathrm{GeV})$ of Perseus cluster with the MAGIC and found that their detection was not associated with the natural pion decay in cluster. Later on, Prokhorov and Churazov (2014) stacked images of 55 galaxy clusters observed in 52.5 months with the FermiLAT in energies greater than $10 \mathrm{GeV}$ and found an excess emission that was highly 
contaminated from the contribution of radio galaxies. Griffin et al. (2014) stacked deep Fermi-LAT observations of Virgo and Perseus clusters, but merely detected some point like emissions that are more likely associated with the radio galaxies. Griffin et al. (2014) also stacked Fermi-LAT images of 78 clusters selected from a broad range of $\mathrm{X}$-ray luminosity and found no excess gamma ray emission in the cluster.

Even if current gamma ray telescopes could detect diffuse gamma emission, they are unable to distinguish between the generating sources, which are hadronic interactions, DM annihilation and IC in clusters. Future gamma ray telescopes such as the Cherenkov Telescope Array (CTA) with unprecedented sensitivity are expected to detect diffuse gamma ray emission in clusters.

\section{§2.6. Particle Acceleration in Clusters}

The re-acceleration of mildly energetic, pre-existing electrons in galaxy clusters is thought to provide sufficient energy to compensate for the extreme energy loss of the electrons and generate diffuse radio emission in clusters. Thus, in this section a brief description about different re-acceleration mechanisms in clusters is given.

Structure formation shocks, also called "diffusive shocks", are known to be the main effective source of CR acceleration in galaxy clusters (Drury, 1983). The structure formation shocks are referred to accretion shocks, merger and flow shocks generated during the cosmic structure formation.

The cooling time of the gas is much longer than the gravitational collapse of the over-densities on scales larger than a Mpc. Therefore, the super-sonic collapse of gas in cosmic filaments generates large scale accretion shocks. Accretion shocks are also generated during cluster merging. Merger shocks are the result of collisions between sub-structures and galaxy groups during the cluster merger and cluster formation. In addition, flow shocks are generated during the gas flow from cosmic filaments and sheets towards the clusters. The combination of the aforementioned shocks can accelerate the CRe to relativistic levels via the diffusive first order Fermi acceleration mechanism (see Miniati et al., 2001 and the references therein).

\section{§2.6.1. Fermi Acceleration Mechanisms}

Fermi acceleration affects the charged particles in astrophysical plasmas in two different ways: the first order Fermi acceleration (Fermi I) and the second order Fermi acceleration (Fermi II). Fermi I mostly accelerates the particles through shocks, whereas the turbulence in the magnetised plasma is responsible for Fermi II acceleration. A brief explanation of the two types of accelerations is given in below. 

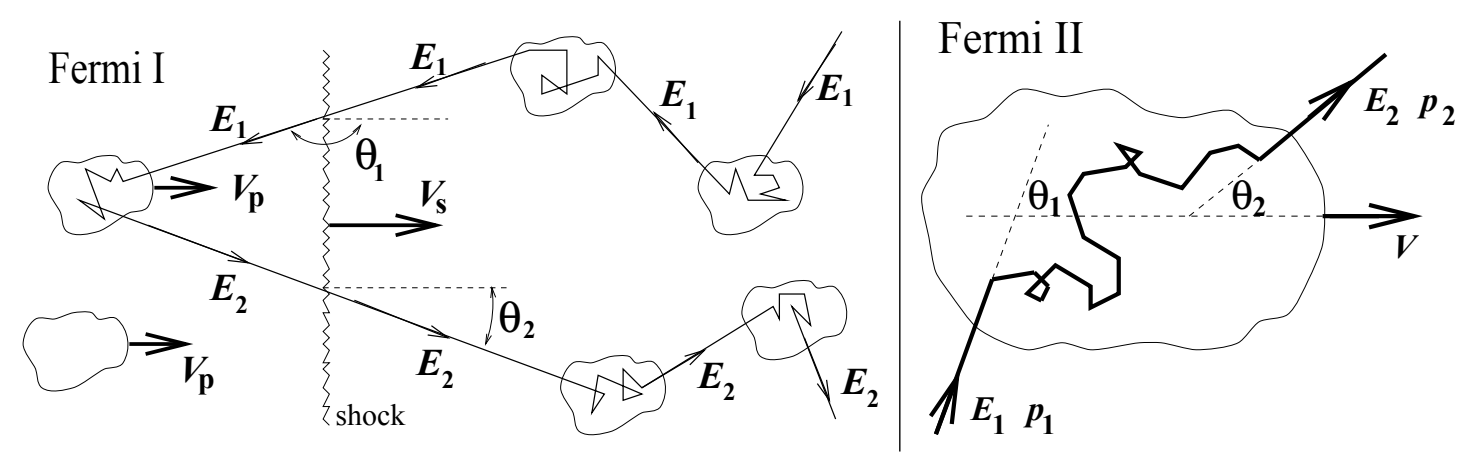

Figure 2.3: Fermi acceleration mechanisms. Left: The particle energy change in the presence of shock in the first order Fermi acceleration. Right: The particle energy change in a magnetised cloud in the second order Fermi acceleration. The images are adapted from Protheroe, 1999.

\section{Fermi I Acceleration}

The collision-less shocks always participate in Fermi acceleration in astronomical plasmas such as the ICM. Generally, a flow on two opposite sides of the shock can be assumed. The pre-shocked or upstream region has the velocity $\mathrm{u}_{1}$, whereas the shocked or downstream region has the velocity of $\mathrm{u}_{2}$. One can assume a non-relativistic testparticle with speed $v_{0}$ in the shock rest frame in upstream side. In the presence of magnetic field, the mentioned test-particle scatters off the other charged particles and starts moving towards the downstream and crosses the shock with speed $\mathrm{v}_{1}$. In the downstream side, the magnetic field scatters the test-particle again to reach speed $v_{2}$ and the test-particle moves towards the upstream and might crosses the bow shock again. During this process, the averaged energy gained by the test-particle per round trip (two successive crossing ) is given by:

$$
\left\langle\frac{\Delta E}{E}\right\rangle \simeq \frac{4}{3} \frac{v_{\mathrm{s}}}{c} \Rightarrow\left\langle\frac{\Delta E}{E}\right\rangle \simeq \frac{4}{3} \beta_{\mathrm{s}}
$$

In this "ping-pong"-like acceleration mechanism, commonly known as first order Fermi shock acceleration, all the particles move towards the downstream in the rest frame of the shock. Hence, the upstream velocity is approximately $\mathrm{v}_{1}=-v_{\mathrm{sh}}$ and the downstream velocity is $\mathrm{v}_{2}=v_{\mathrm{sh}} / 4$. This can be though of as each CR which cross the shock, experiences a flow of charged particles that move towards itself. In the presence of magnetic field in the two sides of the shock, the acceleration increases the energy of the CR. It should be noted that the first order Fermi acceleration merely affects mildly relativistic CRs. However, as mentioned earlier, mildly relativistic electrons are accumulated in time-scales greater than $10^{9} \mathrm{yr}$ in galaxy clusters; thus, these types of electrons are the perfect reservoir to become involved in the acceleration process. The energy spectrum of CRs can be expressed with a power-law relation:

$$
N(E) d E \propto E^{-2} d E .
$$




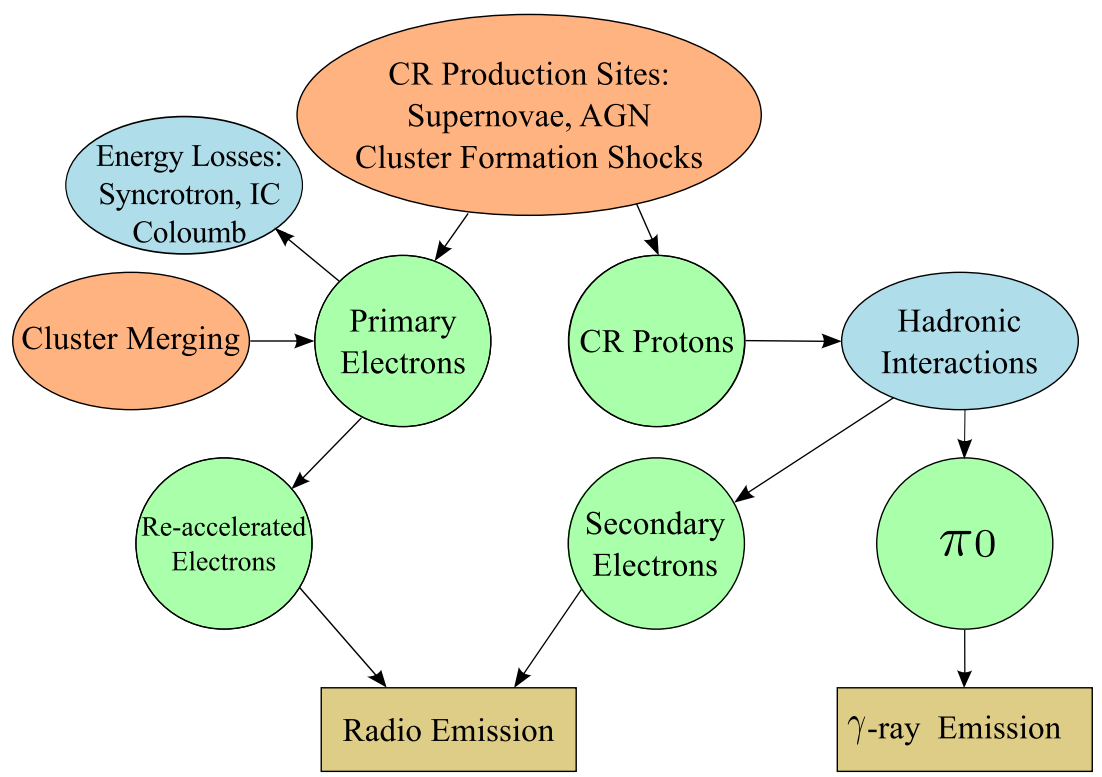

Figure 2.4: An overview of the energy gain and loss of CRs and mechanisms at work to produce radio emission at $\mathrm{GHz}$ regime and $\gamma$-ray emission. The light-orange colour represents the energy supply processes and the light-blue colour represents the energy loss processes. The green colour represents the relativistic particles. The light-brown colour represents the expected radiations in the galaxy clusters according to the halo generation models. The image is adapted from Pfrommer et al. (2008).

\section{Fermi II Acceleration}

Fermi second order acceleration is an effective acceleration mechanism when turbulence occurs in the ICM due to cluster merging. The magnetised plasma in the ICM can move with velocity $v_{\mathrm{cl}}$. The charged particles in the ICM collide with magnetic clouds in the ICM and are then scattered in any random direction. Individual particles might encounter magnetised clouds many times, and in each experience could gain or lose energy. However, on average, the number of incidents leading to the energy gain is slightly higher than the energy loss in the magnetised clouds (Protheroe, 1999). Thus, averaging over many scattering directions and encounters results in an average energy gain and hence the particles experience an acceleration. Total energy gain is given by:

$$
\left\langle\frac{\Delta E}{E}\right\rangle \simeq \frac{4}{3}\left(\frac{v_{\mathrm{cl}}}{c}\right)^{2} \Rightarrow\left\langle\frac{\Delta E}{E}\right\rangle \simeq \frac{4}{3} \beta_{\mathrm{cl}}^{2} .
$$

Since $\beta \ll 1$, total energy gain in Fermi II order is much smaller than Fermi I order. The schematic diagrams of Fermi I and Fermi II acceleration mechanisms are shown in Figure 2.3 from left to right, respectively.

An overview of the energy loss and gain of the CRes and CRps in galaxy clusters is given in Figure 2.4. This figure also shows how secondary electrons and gamma rays are produced in clusters, and which type of emission is expected to be observed in galaxy clusters according to current halo emission mechanisms. 


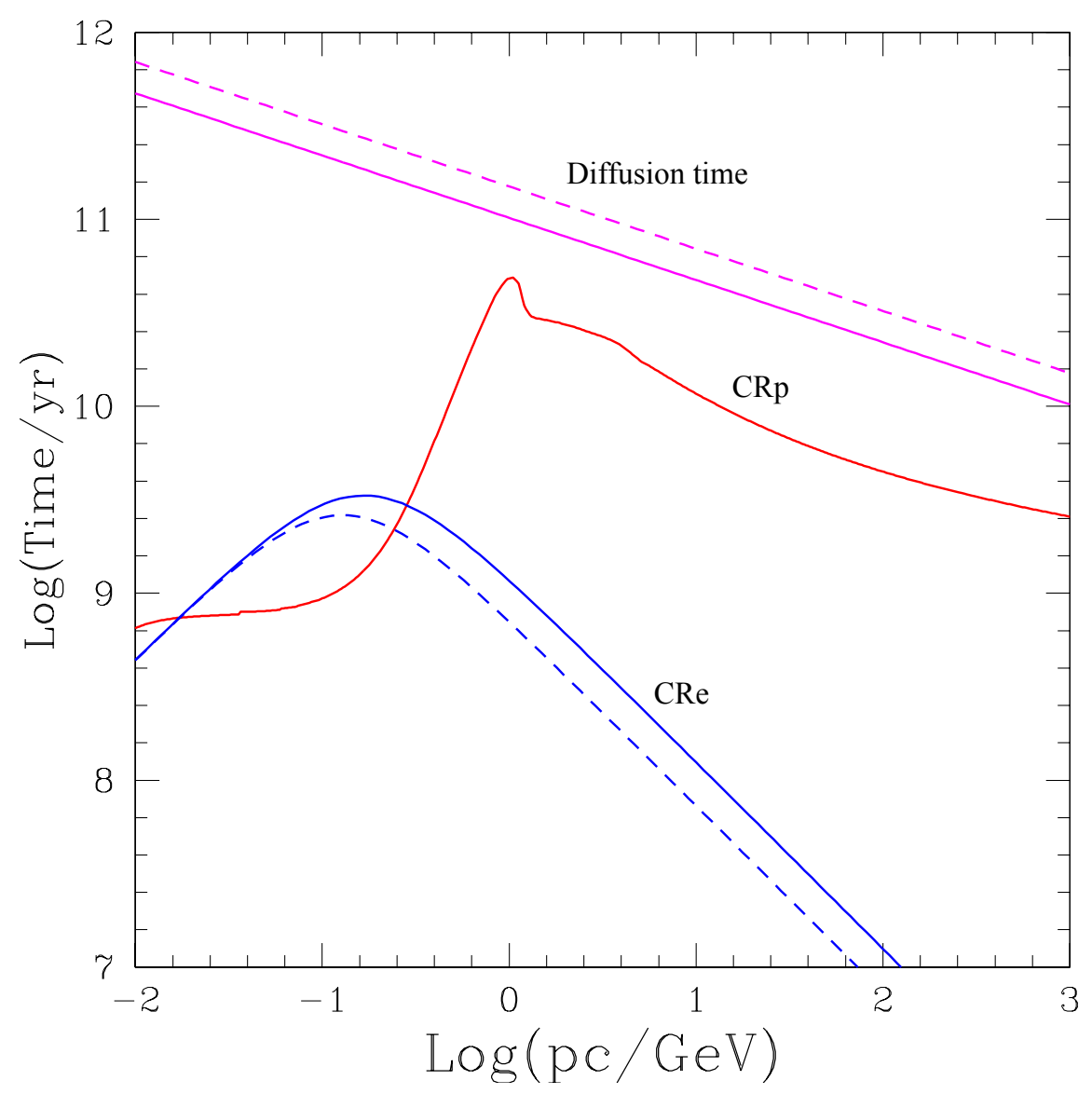

Figure 2.5: The radiative life-time of $\mathrm{CRe}$ and $\mathrm{CRp}$ at $\mathrm{z}=0$ and the magnetic field of $\mathrm{B}=1 \mu \mathrm{G}$ (solid) and $3 \mu \mathrm{G}$ (dashed). The diffusion time is shown with pink solid and dashed lines. The image is adapted from Blasi et al. (2007).

\section{§2.7. Radio Halo Generation Models}

As explained earlier, due to extreme synchrotron and IC losses in clusters, CRes have a fairly short life-time of $\simeq 10^{8} \mathrm{yr}$. The required diffusion time for CRes to propagate in Mpc-scale is at least $10^{9} \mathrm{yr}$, which is an order of magnitude longer than the radiative life-time of CRes. The essential diffusion time for CRes to propagate over the distance $\mathrm{R}$ is given by:

$$
\tau_{d i f f} \simeq 10^{12}\left(\frac{R}{2 \mathrm{Mpc}}\right)^{2}\left(\frac{E}{1 G e V}\right)^{-\frac{1}{3}}\left(\frac{B}{1 \mu \mathrm{G}}\right)^{\frac{1}{3}} \mathrm{yr} .
$$

where $\mathrm{B}$ is the magnetic field and $\mathrm{E}$ is the energy of CRs. For instance, the diffusion time over $1 \mathrm{Mpc}$ distance in $3 \mu \mathrm{G}$ magnetic field strength with electron energy of $1 \mathrm{GeV} \simeq 3.5$ $\times 10^{11} \mathrm{yr}$. This diffusion time is much longer than the CRe life-time in galaxy clusters. This implies that the CRes are either freshly replenished, or the pre-existing CRes are recently re-accelerated to be able to explain the existence of cluster-scale radio halos. The lack of continuous CRe injection or re-acceleration and significant energy loss of 
CRes in clusters result in the occurrence of radio halos with only $\approx$ Gyr life-time. The radiative life-time of CRs and diffusion time are shown in Figure 2.5. Currently there are two proposed models to explain radio halo generation in the galaxy clusters: reacceleration or primary electron model (Schlickeiser et al., 1987; Brunetti et al., 2001), and hadronic or secondary electron model (Dennison, 1980; Blasi and Colafrancesco, 1999).

In the re-acceleration model, low energy primary CRes are re-accelerated through the merging of the clusters. The merger-driven turbulence accelerates CRes via second order Fermi acceleration and CRes gain energy to $10 \mathrm{GeV}$ range and emit synchrotron emission, which is observable at $\mathrm{GHz}$ radio frequencies. Since the decay of turbulence occurs in $\approx 1 \mathrm{Gyr}$, which is a comparatively short time period in the cluster life-time, the re-acceleration should be maintained to avoid CRe cooling. In the hadronic model, the secondary electrons are continuously injected into the ICM via p-p interactions between the CRps and the ICM hadrons. Since the cooling time of primary CRes is very short, the synchrotron emission in this model is strongly dependent on the CRp reservoir and the distribution of the ICM. In addition, the magnetic field amplification and decay can significantly alter the synchrotron emission in this model.

The re-acceleration model implies that all merging clusters should host radio halos. Although all radio halos have been detected in the merging clusters, only small fractions of merging clusters have radio halos. On the other hand, the hadronic model implies that all the galaxy clusters harbour radio halos. This implication is not supported by current radio observations of galaxy clusters. We now discuss strengths and weaknesses of these two models in explaining the observational properties of radio halos.

\section{Re-acceleration model strengths:}

- A bimodal behaviour in the distribution of galaxy clusters with/without halos in terms of their radio luminosity is expected to occur naturally. The low radio luminosity of relaxed clusters and high radio luminosity in disturbed clusters is the result of the lack of, or the existence of turbulent re-acceleration in clusters, respectively (Ensslin et al., 2011 and references therein).

- Turbulence re-accelerates CRes via Fermi second order acceleration, which is a natural phenomenon in the astronomical plasma such as the ICM (Ensslin et al., 2011 and references therein).

- The observed peculiar morphology in some radio halos are the result of recurrent turbulence in the clusters (Ensslin et al., 2011 and references therein).

- The occurrence of radio halos with ultra-steep spectrum and spatially variant steep spectrum of halos is a natural outcome in this model. The observed cut-off and break in the halo spectrum is intrinsically due to the interplay between the energy 
loss and re-acceleration energy gain of CRes (Ensslin et al., 2011 and references therein).

- A strong correlation between the radio halo power and the X-ray luminosity of the parent cluster is expected to emerge naturally (Ensslin et al., 2011 and references therein).

- The lack of radio halos in merger clusters are the result of insufficient re-acceleration due to turbulent decay.

\section{Re-acceleration model weaknesses:}

- Fermi II acceleration has limited efficiency at large scales. Thus, CRes energy loss is inevitable and significant (Ensslin et al., 2011 and references therein).

- Throughout the re-acceleration of the CRe population, the CRps (their cooling time is much longer than CRes), also gain energy in the life-time of the cluster. In addition, it is known that the shock waves in clusters inject hundreds of times more CRps than CRes into the ICM. Thus, due to the boosted level of hadronic interactions, the population of secondary electrons grows dramatically. This can be interpreted that the secondary electrons exceed the primary electrons in galaxy clusters.

- Spectral curvature (due to the re-acceleration) in the power-law spectra is not entirely consistent with the observed radio halos spectra.

- The observed regular morphology of the majority of radio halos is not in agreement with the morphology predicted by turbulence.

\section{Hadronic model strengths:}

- CRps are initially injected by CR sources in the ICM. Therefore, the occurrence of radio halos is a natural phenomenon.

- The observed steep radio halo spectra should occur naturally in this model due to injection of highly-energetic secondary electrons into the ICM.

- The regular morphology of radio halos are easily realised in this model. The distribution of the accumulated long-lived CRps in the clusters are expected to be smooth.

- A radio/X-ray scaling relation under the assumption of $\mu \mathrm{G}$ magnetic field and efficient CRp acceleration is expected in this model.

\section{Hadronic model weaknesses:}


- Radio halos have not been observed in the majority of clusters; more than $50 \%$ of the clusters with high X-ray luminosities do not exhibit any sign of halo emission.

- The spectrum curvature of the well-studied radio halos such as Coma is not consistent with the predicted spectra in the halo simulation. However, it should be noted that in the simulations of the hadronic model, some important parameters such as diffusion and streaming have not been accounted for in the literature.

- The observed steep spectrum at the edges of some radio halos cannot be easily inferred in this model.

It is worthwhile to mention that there are only a few radio halos with multi-frequency data available in the radio regime. Hence, the integrated and spectral map of radio halos cannot yet be studied in great detail. Additionally, the majority of radio halos host multiple radio sources which need to be accounted for when studying the halo properties. The peeling procedure of embedded radio sources is not very accurate and the residual emission is often seen in the peeled radio maps. The remaining artefacts or residual signals can significantly affect the spectral index analysis of the radio halos.

According to the re-acceleration model, X-ray under-luminous clusters are less plausible candidates to host radio halos (Cassano et al., 2006, 2008). The majority of radio halo surveys have so far only observed clusters with high X-ray luminosity. However, there are a handful of low X-ray luminosity clusters with radio halos in the literature. Thus, it is not clear that the lack of halos is a real phenomenon or it is due the selection effects in current radio halo surveys. Halo surveys of a sample of clusters with homogeneous X-ray luminosities are required to better understand the lack of radio halos in clusters with low X-ray luminosities. In order to test the observational implications of the radio halo generation models, multi-frequency radio observation of clusters with superior sensitivity and larger bandwidth are essential.

It is known that the interplay between the primary CRes and the merger driven turbulence leads to switching on/off the radio halos, but fading the hadronically driven radio halos is not yet well understood. Multiple components such as the ICM density profile, cluster dynamics and magnetic field profile can heavily affect the CR propagation in the clusters, thus, CR diffusion is poorly known and more reliable data and accurate numerical simulations are required for better understanding. CR streaming in clusters is generally accepted to be due to the motion along the magnetic field lines and diffusion via scattering the ICM plasma waves.

According to recent numerical simulations of halos based on the combination of the aforementioned models, and given that neither of the generation models are entirely successful in explaining the observational data, Pfrommer et al. (2008) proposed a combined model in which the central part of a radio halo is generated by a hadronic 
interaction mechanism due to the higher CR density in the core and shock waves on the outskirts of radio halos, which have higher Mach numbers, can effectively accelerate the primary electrons.

Ensslin et al. (2011) investigated the distribution and advection of CRs in galaxy clusters. They found that the CR radial profile is flat and turbulence can enhance CR density in the central regions of the cluster. The combination of higher magnetic field intensity, centrally peaked ICM density and the presence of more energetic plasma waves in re-accelerating the CRs, leads to a centrally boosted CRp profile in clusters. Consequently, radio halos emerge naturally in galaxy clusters regardless of the generation model. They also pointed out that lack of turbulence could suppress radio and gamma ray emission in clusters. Following this research, Brunetti et al. (2012) investigated two scenarios including only secondary electrons. They first considered a simple pure hadronic model in which the secondary electrons are continuously replenished (with stationary spectrum) in the cluster to generate and maintain a Coma-like halo. They used all the datasets available for the Coma cluster and investigated the spectral index and radio halo brightness profile to estimate the CRp profile inside the cluster. They found that the predicted gamma ray emission exceeded the current Fermi-LAT upper limits in the pure hadronic model. In the case that they confined the gamma ray to the detection limit of Fermi-LAT, a higher magnetic field was necessary to generate radio halo, which is inconsistent with current observations. Hence, they argued that the pure hadronic model could not generate radio halos. They also investigated a more complicated scenario in which the secondary electrons are re-accelerated by magneto-hydro dynamics (MHD) turbulence via the Fermi II re-acceleration mechanism. They pointed out that radio halos with current morphology and spectral curvature could be generated in this scenario, while the magnetic field and gamma ray emission remain consistent with observations. However, they also noted that this model requires that CRps be extended at larger radii.

Although Fermi II re-acceleration of either primary and secondary CRes alongside the $\mathrm{CR}$ streaming can explain the current radio halo observations to a great extent, the actual electron population (primary or secondary) associated with the radio halos is indistinguishable and the origin of electron seeds are as yet unknown (Ensslin et al., 2011; Brunetti et al., 2012; Pinzke et al., 2015). 


\section{§3. Data Reduction and Imaging of CABB Data}

As early users of $\mathrm{CABB}$ following its upgrade, it was necessary to trial several different reduction strategies for $\mathrm{CABB}$ data. This chapter documents the results of those trials. It is not intended to be a comprehensive data reduction guide to the ATCA; rather to give an overview of the process needed for wideband ATCA data with a high fractional bandwidth.

\section{§3.1. Australia Telescope Compact Array}

The Australia Telescope Compact Array (ATCA) is an Earth rotation aperture synthesis radio interferometer located west of Narrabri in Australia. The telescope is comprised of six identical antennas with a $22 \mathrm{~m}$ diameter dish (Frater et al., 1992). The ATCA with its six antennas is shown in Figure 3.1. The relative location of the six antennas is called the array configuration and each configuration has 15 baselines where the shortest and longest baselines are $31 \mathrm{~m}$ and $6 \mathrm{~km}$, respectively. There are five mobile antennas which could be positioned at 44 different locations along a $3 \mathrm{~km}$ long east-west track. The other antenna (known as antenna 6) is fixed $3 \mathrm{~km}$ away from the western end of the east-west track to provide the longest baseline. There is also a $214 \mathrm{~m}$ long north-south track, which is used to create three hybrid configurations (also known as compact configurations). There are 17 configurations available for observing throughout the year; however, only the most requested arrays are typically offered. The synthesised beam width for observing wavelength $\lambda$ is $\mathrm{u}^{-1} \times \mathrm{u}^{-1} \sec \delta$ where is $\mathrm{u}^{-1} \approx \frac{0.9 \lambda}{6}[\mathrm{rad}]$ and $\delta$ is the declination of the source.

The backend system of the ATCA has been recently upgraded to the Compact Array

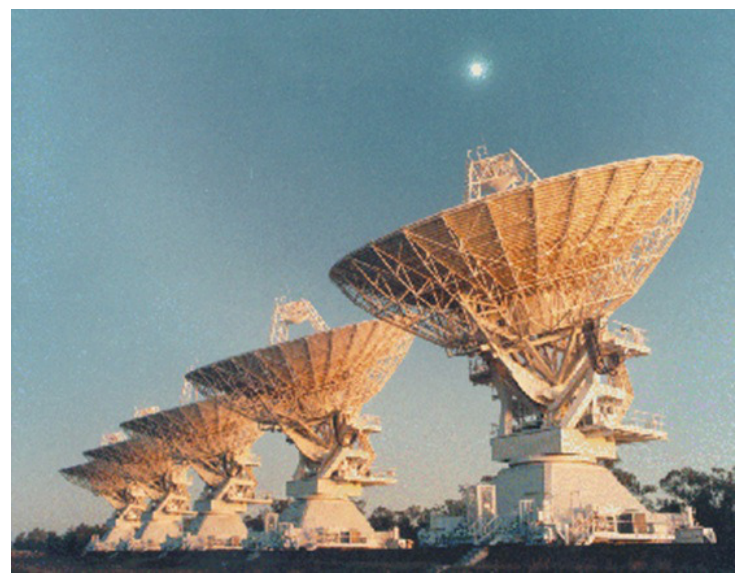

Figure 3.1: Australia Telescope Compact Array (ATCA) in Narrabri, Australia. 
Broadband Backend (CABB) and with the aim of increasing the observing bandwidth from $128 \mathrm{MHz}$ to $2 \mathrm{GHz}$ (Wilson et al., 2011). This improvement has resulted in better uv-coverage and, consequently, higher sensitivity. In addition, it has reduced the required observing time to achieve desired sensitivities in continuum observations. The sensitivity is expected to improve by at least a factor of four; however, the practical sensitivity enhancement is significantly less than expected values at $\mathbf{1 6} \mathbf{~ c m}$. This is mainly because of the extreme Radio Frequency Interference (RFI) that now occurs particularly in the frequency range of 1.1-3.1 GHz. Although the RFI sources such as "mid-week" RFI at $1265 \mathrm{MHz}$ and $1310 \mathrm{MHz}, \mathrm{L}-$ band Satellites at 1525-1660 MHz and, the Terrestrial Microwave Link at $1427-1535 \mathrm{MHz}$ and $1700-1900 \mathrm{MHz}$, respectively have limited bandwidth, a significant portion of the CABB band at $2.1 \mathrm{GHz}$ is affected. The sensitivity at the other observing bands is not significantly affected by RFI. The characteristics of the radio data observed at $2.1 \mathrm{GHz}(16 \mathrm{~cm})$ are given below:

$\begin{array}{ll}\text { Frequency range } & 1.1-3.1 \mathrm{GHz} \\ \text { Number of antennas } & 6 \\ \text { Number of baselines } & 15 \\ \text { Primary Beam } & 15^{\prime}-42^{\prime} \\ \text { Synthesised Beam } & 3^{\prime \prime}-9^{\prime \prime} \\ \text { Central Frequency } & 2.1 \mathrm{GHz} \\ \text { Flux Sensitivity (10 mins) } & 0.03 \mathrm{mJy} \mathrm{beam}^{-1} \\ \text { Flux Sensitivity (12hrs) } & 4 \mu \mathrm{Jy} \mathrm{beam}^{-1}\end{array}$

\section{§3.2. CABB Data Reduction}

The high fractional bandwidth of the CABB data at $2.1 \mathrm{GHz}$ has raised issues, particularly in the imaging process. The synthesised beam size varies dramatically across the CABB band at $2.1 \mathrm{GHz}$, limiting the ability of the deconvolution tasks to determine the spectral variation of the beam over the full band. In addition, our findings suggest that accurate data reduction of the whole $2 \mathrm{GHz}$ bandwidth simultaneously is problematic due to the change of system temperature as function of frequency. To overcome these issues we suggest the sub-division of the full CABB band into a minimum of four, $500 \mathrm{MHz}$ sub-bands. The data reduction process described in the following should be carried out for each sub-band.

Data reduction process is shown in Figure 3.2. The observed ATCA data are reduced by the MIRIAD software which was initially developed by Sault et al. (1995) and exclusively designed for the Compact Array. The observed data with the ATCA is stored in "RPFITS" format, which is recognised by MIRIAD, and the data file can be loaded into MIRIAD by a task called "atlod". For the purpose of sub-division of the 


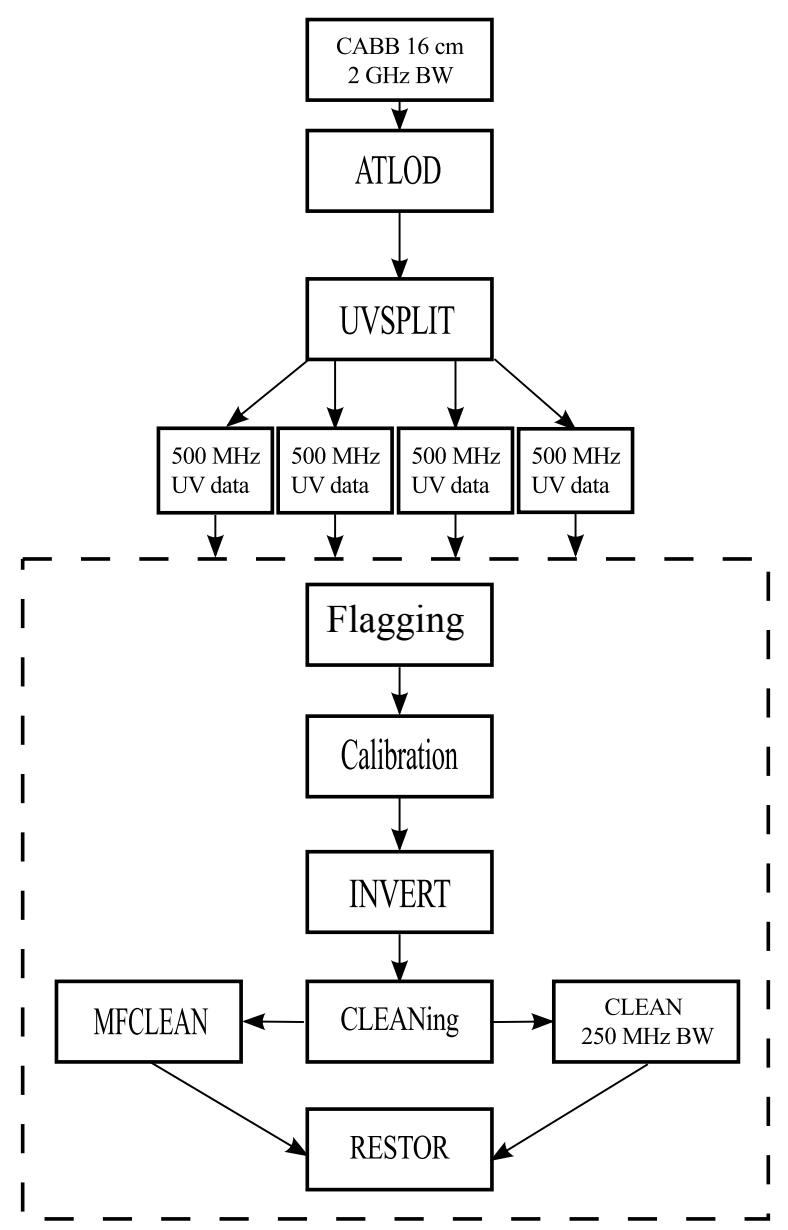

Figure 3.2: Data reduction flow chart in MIRIAD. These routines in the dashed box should be carried out for each sub-divided (e.g. $500 \mathrm{MHz}$ ) uv-dataset.

CABB data, the parameter maxwidth $=0.5$ (or less) can be set in the "uvsplit" task The first stage of any data reduction is removal of spurious terrestrial signals from RFI. The term "flagging" refers to discarding observed data that are affected by RFI. Noninteractive flagging tasks such as uvflag, pgflag, blflag and tvclip are implemented in MIRIAD to flag the data. Some interactive flagging tasks such as "blflag" and "pgflag" are designed to visually inspect the data and discard the RFI. Non-interactive flagging tasks are faster in editing visibilities and removing the RFI; however, they are prone to inaccuracies, and RFI-free visibilities could unintentionally be removed during the flagging procedure. The flagging task, "pgflag", offers interactive and automated flagging simultaneously with sufficient accuracy. This task can be used first to eliminate RFI from raw data. Task "uvflag" is useful when a large part of the spectrum or baseline is required to be removed due to RFI.

Calibration of instrumental errors should be accounted for after the flagging process. Tasks "mfcal" and "gpcal" in MIRIAD are used to calibrate the flagged data. According to interferometry, radio emission is collected by the receiver of each antenna. The collected data is amplified, correlated, and averaged by a complex correlator. Recorded 
visibilities collected from each antenna pair are called "observed" visibilities. Generally, received visibilities differ from actual visibilities. The purpose of calibration is to recover true visibilities from observed data. To estimate correct visibilities, the complex gains (which are complex visibilities with amplitude and phase terms) must be calibrated. Typically, three types of calibrations are required to correct for instrumental errors and recover the actual visibilities at $16 \mathrm{~cm}$. These are:

\section{Flux}

Flux calibration is required to estimate amplitude errors in the observed visibilities. The source of amplitude errors is the noise that contributes to the system temperature, $T_{\text {sys }}$. An effective system temperature for a pair of antenna is the product of system temperature associated with each antenna. The observed flux is the product of the measured system temperature and true flux,

$$
T_{s y s_{(e f f)}}=T_{s y s_{(i, j)}}=T_{s y s_{(i)}} T_{s y s_{(j)}},
$$

where $\mathrm{i}$ and $\mathrm{j}$ represent the antenna number. A flux calibrator should be a fairly strong point source with virtually constant flux during the Earth's rotation. Since the amplitude of the flux calibrator is known, the noise in the system temperature can be estimated from raw visibilities to obtain a flux scale.

\section{Bandpass}

Bandpass calibration is necessary to correct the complex gain variations as a function of frequency. The shape of the bandpass is determined by the band-pass filters that have a flat response to frequency. Most radio telescopes such as the ATCA have rectangular bandpass filters. The variation of the flux as a function of frequency (bandpass shape) for the raw data is shown in the left panel of Figure 3.3. The observed bandpass shape is the multiplication of the bandpass flat response and the true spectrum of the source. To create calibration tables, observation of a strong point source with known flux and spectrum is required. The final spectrum after bandpass calibration is shown in the right panel of Figure 3.3.

\section{Phase}

The phase of the visibilities indicates the position of the observed radio sources on the plane of sky; therefore, an accurate phase calibration of observed visibilities is vital. Slight variations and drifts in the receiver gain while collecting the data induces phase error. In addition, different paths in the electronic components result in variation of phase in the correlator input. However, the major source of phase error is atmospheric instabilities during the observation. For phase calibration, frequent observation of a Phase calibrator is required to identify atmospheric 

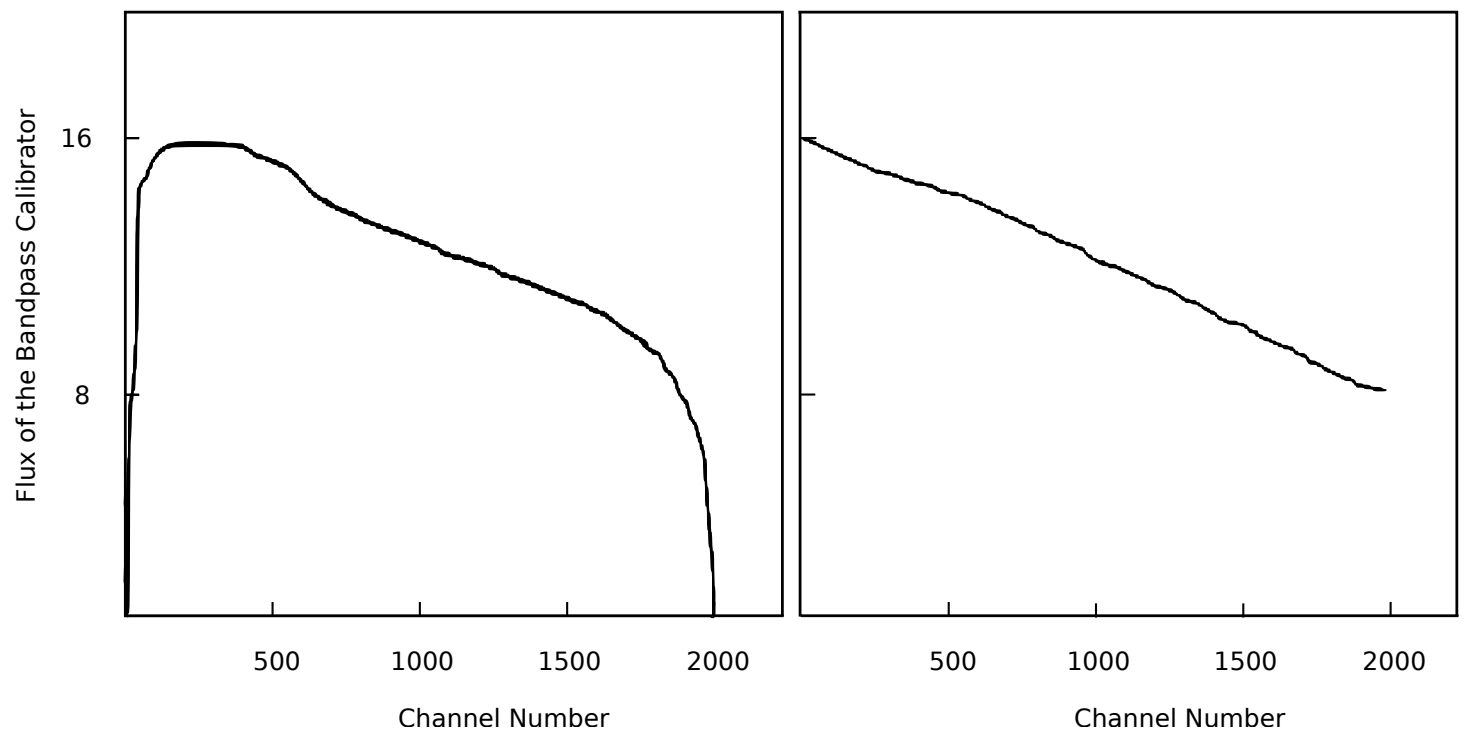

Figure 3.3: The spectrum of the primary calibrator observed at $2.1 \mathrm{GHz}$ with $2 \mathrm{GHz}$ bandwidth. Left panel: the spectrum of the non-calibrated (bandpass) PKS B1934-638. Right panel: the final spectrum after bandpass calibration for PKS B1934-638.

phase errors. The phase calibrator is typically called the secondary calibrator, and must be close to the target source to probe the same atmospheric path during observation.

The flux and bandpass calibration steps are carried out by observing PKSB1934-638 (also known as the primary calibrator). This calibrator is a strong, unresolved and unpolarised point source, which makes it an ideal flux and bandpass calibrator. The total flux of PKSB1934-638 is $14.9 \mathrm{Jy}$ at $1.4 \mathrm{GHz}$. All the required calibration steps are shown in the flowchart in Figure 3.4. The MIRIAD task "mfcal" determines the bandpass and flux calibration transfer functions using the primary calibrator, PKSB1934638.

For the polarimetry, deriving the polarisation leakages are vital. The task "gpcal" in MIRIAD solves complex antenna gains and polarisation leakages using an unpolarised point source calibrator. In the case of pre-CABB data with a narrow bandwidth of only $128 \mathrm{MHz}$, the polarisation leakages are constant across the bandwidth. However, in $\mathrm{CABB}$ mode, which has a much wider bandwidth $(2 \mathrm{GHz})$, polarisation leakages vary across the full band. To obtain a precise solution for gain and polarisation leakages, it is recommended to use the "nfbin" parameter in task gpcal to obtain frequencyindependent calibration tables. To generate the phase calibration transfer functions, first the bandpass solution of the primary calibrator should be copied into the secondary calibrator. The option "qusolve" should be used in "gpcal" to determine Stokes Q and $\mathrm{U}$ parameters of the secondary calibrator. Appropriate parallacic angle coverage is required to have a reasonable approximation for the polarisation values. In our experience, at least five cuts of observations are essential for sufficient parallactic angle coverage. 


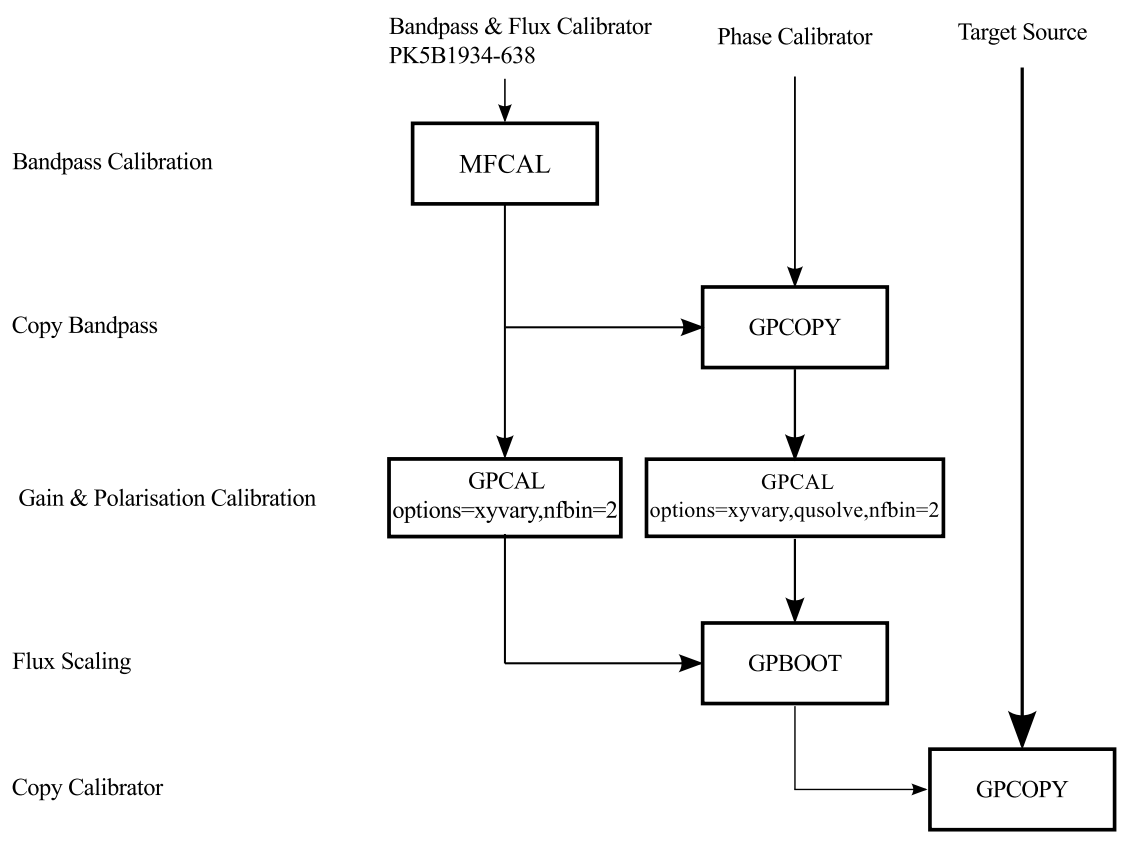

Figure 3.4: The calibration process in MIRIAD for CABB uv-data observed at $2.1 \mathrm{GHz}$. The flowchart is adapted and modified from MIRIAD user manual.

In the case of insufficient secondary calibrator data, polarisation leakages and Stokes parameters can be obtained from the primary calibrator with an extra option parameter "nopol" in task gpcal. The calibration process concludes by copying all the calibration tables from the secondary calibrator into the target source. Standard imaging process can then be used to obtain the final CLEANed radio image.

\subsection{Imaging of CABB data}

CABB imaging differs slightly from pre-CABB. The size of synthesised beam rapidly changes as a function of frequency at $2.1 \mathrm{GHz}$ across the full $\mathrm{CABB}$ band; hence, multifrequency CLEANing (MFCLEAN) should be used to CLEAN dirty maps. Unfortunately, the MFCLEAN task is unable to calculate the spectral beam size across the entire $2 \mathrm{GHz}$ band. Therefore, as mentioned earlier, the full bandwidth sub-division treatment is required to overcome this issue. The CLEANing of the $500 \mathrm{MHz}$ sub-band data can be done in two ways: 1) using the MFCLEAN task to CLEAN the entire (maximum) $500 \mathrm{MHz}$ sub-band; 2) re-divide each of the $500 \mathrm{MHz}$ sub-band uv-data into $250 \mathrm{MHz}$ and CLEAN separately using the task "CLEAN". These approaches allow us to avoid beam spectral variation even across the entire $500 \mathrm{MHz}$ bandwidth and CLEAN a larger region of the dirty map without setting multiple CLEAN boxes. In addition, we find that the latter is the only suitable approach if there is a strong point source (total flux $>100$ $\mathrm{mJy}$ ) in the field. The imaging process should be carried out for each of the sub-divided $500 \mathrm{MHz}$ uv datasets. In the next step, all the sub-images should be convolved to the 
resolution of the lowest frequency sub-band. Eventually, all four sub-band images are linearly mosaiced together using the task "linmos" to enhance the sensitivity of the final image.

\subsection{Summary}

In this chapter, we presented data reduction and imaging of the $\mathrm{CABB}$ data in practice. We discussed the issues around the high fractional bandwidth at $2.1 \mathrm{GHz}$ in the data reduction procedure. We noticed that in practice, the calibration and imaging routines for $\mathrm{CABB}$ data should be altered to overcome the issues raised by the large fractional bandwidth. We concluded that sub-division of the $2 \mathrm{GHz}$ bandwidth into $500 \mathrm{MHz}$ sub-band uv-data is a plausible solution to obtain more accurate calibration solutions and enhanced images. All the data reduction, calibration and imaging routines that are explained in this chapter were used to obtain the radio data presented throughout this thesis. 



\section{§4. Radio Imaging of the REXCESS Clusters Sub- sample}

\section{§4.1. Radio Halo Observations in Galaxy Clusters}

Galaxy clusters are the largest virialised structures and harbour hundreds to thousands of galaxies which are held together by the gravitational potential of the parent cluster. In addition to the galaxies, all clusters contain an inter-cluster plasma which is a hot gas known as inter cluster medium (ICM). Galaxy clusters also contains highly relativistic electrons with Lorentz factors $\gamma>1000$. These highly energetic electrons in the ICM are known to have non-thermal radio emission.

In the past few decades, deep radio observations of galaxy clusters have resulted in the detection of cluster-scale, extended, diffuse synchrotron emission known as radio halos. The number of detections of this type of object is increasing due to utilisation of more sensitive radio telescopes. Despite decades of studying radio halos, the generation mechanisms of radio halos remain unclear. As mentioned in $\S 2.7$, there are two competing models to describe the origin of radio halo occurrence in clusters; the reacceleration model also known as the primary model and the hadronic model also known as secondary model. In the re-acceleration model, it is believed that merger-driven turbulence re-accelerates the primary CRes in the magnetised ICM and triggers radio halo occurrence (Buote, 2001; Cassano et al., 2010; Brown et al., 2011; Basu, 2012b). Additionally, the hadronic model (Dennison, 1980; Vestrand, 1980) can also account for radio halo generation. It is known that relativistic CRps have effective proton-proton collisions in all galaxy clusters and ultra-relativistic electrons are the by-product of these interactions. The fact that hadronic interactions are an ongoing phenomena implies that all clusters should host radio halos at some stage during the cluster's life-time. However, this prediction has not yet supported by radio halo detections. To date, radio halos have only been detected in clusters with minor to major merger activity and high X-ray luminosity. Thus, there is a growing consensus in favour of the primary model as the major mechanism at work in radio halo generation. However, it should be noted that the majority of radio halo surveys have only investigated massive and mostly merging clusters based on the speculation that the chance of halo detection in dynamically active clusters with boosted X-ray luminosity is increased. Thus, current radio halo surveys are significantly biased towards detecting more radio halos in massive, merging clusters with high X-ray luminosity. Consequently, an unbiased statistic of the detection 
rate across all clusters are not yet known. Additionally, most of the halo surveys have utilised the telescopes such as the Giant Metrewave Radio Telescope (GMRT), the Very Large Array (VLA), the Westerbork Synthesis Radio Telescope (WSRT) and the Green Bank Telescope (e.g. Clarke and Ensslin, 2006b, Giovannini et al., 2009, Farnsworth et al., 2013), therefore, clusters in the Southern sky with declination below $-30^{\circ}$ are mostly remained unexplored.

Given the imperfections of current radio halo surveys, in this work we opted for conducting a radio halo survey to probe the most unexplored region of the Southern sky in an unbiased fashion. For the purpose of this experiment we have chosen a subsample of 15 galaxy clusters with $-70^{\circ}<\delta<-20^{\circ}$ (this is the declination range to obtain reasonable beam and uv-coverage in the ATCA observations) from the Representative XMM-Newton Cluster Structure Survey (REXCESS, Böhringer et al., 2007) and used the superior sensitivity of the ATCA in CABB mode to exploit the benefits of the variety of available short baselines to search for Mpc-scale radio emission. The REXCESS is an ideal cluster sample to conduct a radio halo survey since it has no bias in morphology and also contains clusters with a wide-range of X-ray luminosities. In addition, the majority of clusters have very deep X-ray observation ( $>30 \mathrm{ks}$ ) with the XMM-Newton. In this chapter, details of previously observed Pre-CABB and new CABB observations are given. Additionally, all the details of low and high resolution images are given. The high resolution images and postage stamps of each observed cluster and the classification of individual detected source are also presented. In this chapter we assume a $\Lambda$ CDM cosmology with $\mathrm{H}_{0}=67 \mathrm{kms}^{-1} \mathrm{Mpc}^{-1}, \Omega_{m}=0.32$ and $\Omega_{\Lambda}=0.68$ (Planck Collaboration et al., 2013).

\section{§4.2. The REXCESS Sample}

The REXCESS sample was initially introduced by Böhringer et al. (2007). They have chosen 33 local galaxy clusters with $\mathrm{z}<0.2$ from the parent ROSAT-ESO Flux-Limited X-Ray (REFLEX) cluster catalogue (Böhringer et al., 2004). All the galaxy clusters have $\mathrm{L}_{x}$ in range of $0.407-20 \times 10^{44} \mathrm{~h}_{50}^{2} \mathrm{erg} \mathrm{s}^{-1}$ in ROSAT band $(0.1-2.4 \mathrm{keV}$ at the rest frame). In the REXCESS cluster sample, selection effects in the X-ray luminosity has not been noticed and it is considered morphologically unbiased. More details of the REXCESS cluster sample can be found in Böhringer et al. (2007). Figure 4.1 shows the $\mathrm{L}_{X}$ and redshift distribution of the REFLEX catalogue. The red filled dots represent the REXCESS clusters and the red dots with blue circle around them are the clusters presented in this work. All the X-ray images were reduced by (Böhringer et al., 2007) and all final X-ray images were provided to us by Dr Gabriel Pratt. 


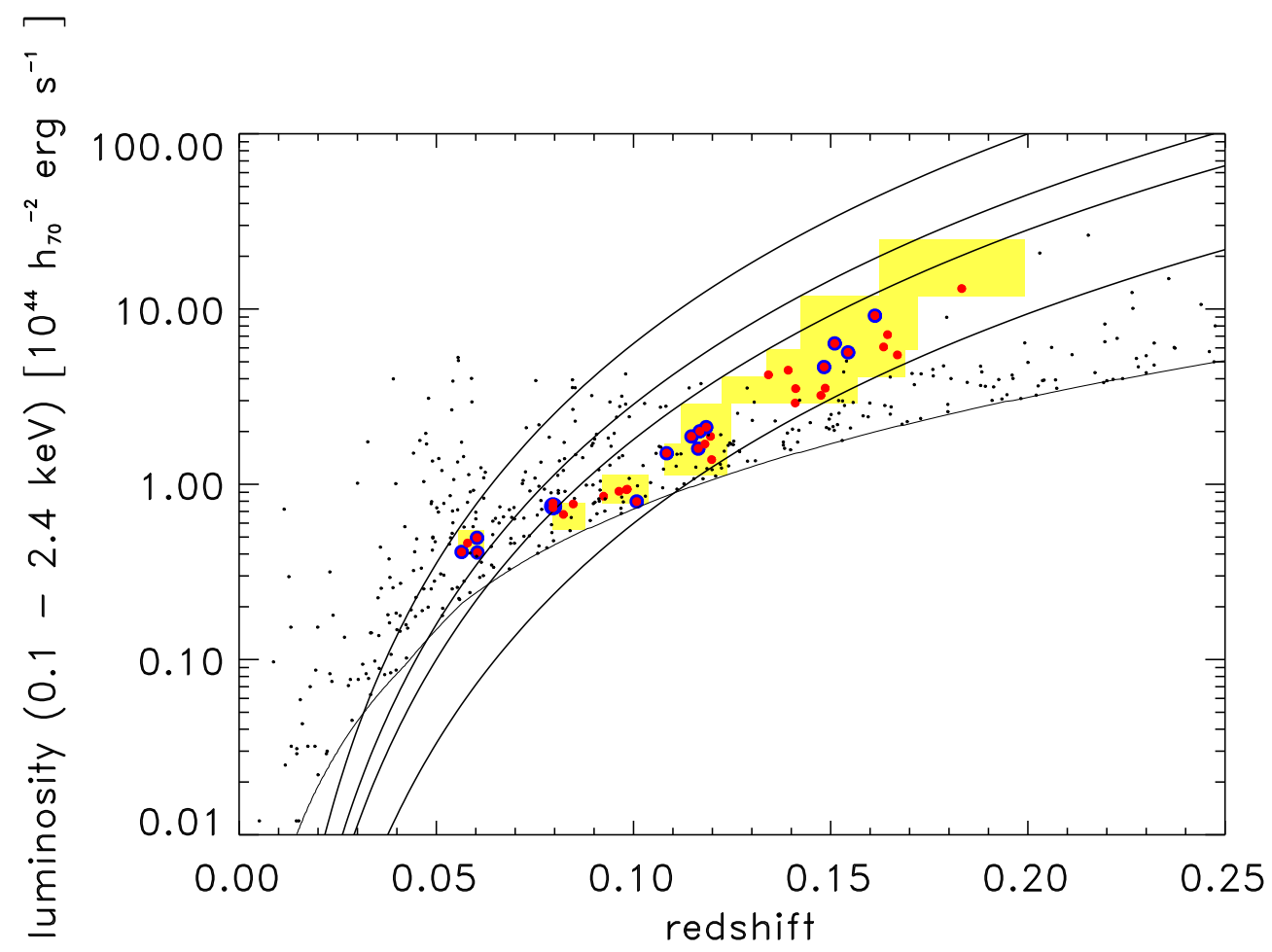

Figure 4.1: The $\mathrm{L}_{X}-\mathrm{z}$ distribution of the clusters in the REFLEX sample. The red filled dots represent the clusters in the REXCESS. The image is adapted from Böhringer et al. (2007). The red dots enclosed with blue circles were observed with the ATCA and are presented in this work.

\section{§4.3. Pre-CABB Radio Observations and Low Resolution Images}

The pre-CABB observations of the REXCESS clusters sub-sample were carried out by Dr Melanie Johnston-Hollitt in June 2007 (EW352 config), July and August 2007 (750B config). These configurations, sensitive to largest spatial scale of $24^{\prime}$ at $1.4 \mathrm{GHz}$ are optimal to detect the Mpc-scales diffuse radio emission at $1.4 \mathrm{GHz}$ at the redshift range of our observed sub-sample. The pre-CABB radio observations were performed at two central frequencies of 1.34 and $1.43 \mathrm{GHz}$ with $128 \mathrm{MHz}$ bandwidth. However, the effective bandwidth in pre-CABB observations is $96 \mathrm{MHz}$ at $1.4 \mathrm{GHz}$, therefore the final combined datasets had a $184 \mathrm{MHz}$ bandwidth at central frequency of $1.39 \mathrm{GHz}$.

Here we clarify that data collection and reduction of all pre-CABB datasets have been performed prior to this work and we have only used the available pre-reduced pre$\mathrm{CABB}$ data and re-processed the images. Details of the pre-CABB radio observations with the ATCA are given in Table 4.1.

Tapered low resolution pre-CABB images revealed that there were three clusters with diffuse radio emission candidates of which one was a radio halo candidate in Abell 3888 and the two remaining were radio relic candidates in clusters Abell 3771 and RXCJ0145.0-5300, respectively. The low resolution $1.4 \mathrm{GHz}$ pre-CABB data are pre- 
Table 4.1: Details of the pre-CABB ATCA radio observations of our REXCESS sub-sample.

\begin{tabular}{|c|c|c|c|c|c|c|c|}
\hline \multirow{3}{*}{$\begin{array}{l}\text { Cluster } \\
\text { RXCJ0006.0-3443 }\end{array}$} & \multirow{2}{*}{$\begin{array}{l}\text { Obs Dates } \\
\text { Pre-CABB } \\
\text { 06-17 June } 2007\end{array}$} & \multirow{2}{*}{$\begin{array}{c}\text { Int Time } \\
\text { Mins } \\
330\end{array}$} & \multirow{2}{*}{$\begin{array}{r}\text { Freq } \\
\text { GHz } \\
1.4\end{array}$} & \multirow{2}{*}{$\begin{array}{c}\begin{array}{c}\text { Bandwidth } \\
\mathrm{MHz}\end{array} \\
128\end{array}$} & \multirow{2}{*}{$\begin{array}{l}\text { Config } \\
\text { EW352 }\end{array}$} & \multicolumn{2}{|c|}{$\begin{array}{l}\text { Pointing } \\
\text { RA, Dec }\end{array}$} \\
\hline & & & & & & 00:06:03.00 & $-34: 43: 27.00$ \\
\hline & 26-31 July 2007 & 310 & 1.4 & 128 & $750 \mathrm{~B}$ & 00:06:03.00 & $-34: 43: 27.00$ \\
\hline \multirow[t]{2}{*}{ RXCJ0049.4-2931 } & 06-17 June 2007 & 365 & 1.4 & 128 & EW352 & $00: 49: 24.00$ & $-29: 31: 28.00$ \\
\hline & 26-31 July 2007 & 282 & 1.4 & 128 & $750 \mathrm{~B}$ & $00: 49: 24.00$ & $-29: 31: 28.00$ \\
\hline \multirow[t]{2}{*}{ RXCJ0145.0-5300 } & 06-17 June 2007 & 364 & 1.4 & 128 & EW352 & $01: 45: 02.30$ & $-53: 00: 50.00$ \\
\hline & 26-31 July 2007 & 240 & 1.4 & 128 & $750 \mathrm{~B}$ & $01: 45: 02.30$ & $-53: 00: 50.00$ \\
\hline \multirow[t]{2}{*}{ RXCJ0211.4-4017 } & 06-17 June 2007 & 288 & 1.4 & 128 & EW352 & $02: 11: 25.50$ & $-40: 17: 12.00$ \\
\hline & 26-31 July 2007 & 280 & 1.4 & 128 & $750 \mathrm{~B}$ & $02: 11: 25.50$ & $-40: 17: 12.00$ \\
\hline \multirow[t]{2}{*}{ RXCJ0225.1-2928 } & 06-17 June 2007 & 228 & 1.4 & 128 & EW352 & $02: 25: 10.50$ & $-29: 28: 26.00$ \\
\hline & 26-31 July 2007 & 320 & 1.4 & 128 & $750 \mathrm{~B}$ & $02: 25: 10.50$ & $-29: 28: 26.00$ \\
\hline \multirow[t]{2}{*}{ RXCJ0345.7-4112 } & 06-17 June 2007 & 270 & 1.4 & 128 & EW352 & $03: 45: 45.70$ & $-41: 12: 27.00$ \\
\hline & 26-31 July 2007 & 280 & 1.4 & 128 & $750 \mathrm{~B}$ & $03: 45: 45.70$ & $-41: 12: 27.00$ \\
\hline \multirow[t]{2}{*}{ RXCJ0616.8-4748 } & 06-17 June 2007 & 390 & 1.4 & 128 & EW352 & $06: 16: 53.60$ & $-47: 48: 18.00$ \\
\hline & 26-31 July \& 1-3 Aug 2007 & 332 & 1.4 & 128 & $750 \mathrm{~B}$ & $06: 16: 53.60$ & $-47: 48: 18.00$ \\
\hline \multirow[t]{2}{*}{ RXCJ0645.4-5413 } & 06-17 June 2007 & 390 & 1.4 & 128 & EW352 & $06: 45: 29.30$ & $-54: 13: 08.00$ \\
\hline & 26-31 July \& 1-3 Aug 2007 & 360 & 1.4 & 128 & $750 \mathrm{~B}$ & $06: 45: 29.30$ & $-54: 13: 08.00$ \\
\hline \multirow[t]{2}{*}{ RXCJ1236.7-3354 } & 06-17 June 2007 & 420 & 1.4 & 128 & EW352 & $12: 36: 44.70$ & $-33: 54: 10.00$ \\
\hline & 26,31 July 2007 & 400 & 1.4 & 128 & $750 \mathrm{~B}$ & $12: 36: 44.70$ & $-33: 54: 10.00$ \\
\hline \multirow[t]{2}{*}{ RXCJ2014.8-2430 } & 06-17 June 2007 & 376 & 1.4 & 128 & EW352 & $20: 14: 49.70$ & $-24: 30: 30.00$ \\
\hline & 26-31 July 2007 & 352 & 1.4 & 128 & $750 \mathrm{~B}$ & $20: 14: 49.70$ & $-24: 30: 30.00$ \\
\hline \multirow[t]{2}{*}{ RXCJ2023.0-2056 } & 06-17 June 2007 & 420 & 1.4 & 128 & EW352 & $20: 23: 01.60$ & $-20: 56: 55.00$ \\
\hline & 26-31 July 2007 & 240 & 1.4 & 128 & $750 \mathrm{~B}$ & $20: 23: 01.60$ & $-20: 56: 55.00$ \\
\hline \multirow[t]{2}{*}{ RXCJ2129.8-5048 } & 06-17 June 2007 & 450 & 1.4 & 128 & EW352 & $21: 29: 51.00$ & $-50: 48: 04.00$ \\
\hline & 26-31 July 2007 & 310 & 1.4 & 128 & $750 \mathrm{~B}$ & $21: 29: 51.00$ & $-50: 48: 04.00$ \\
\hline \multirow[t]{2}{*}{ RXCJ2149.1-3041 } & 06-17 June 2007 & 360 & 1.4 & 128 & EW352 & $21: 49: 07.40$ & $-30: 41: 55.00$ \\
\hline & 26,31 July 2007 & 320 & 1.4 & 128 & $750 \mathrm{~B}$ & 21:49:07.40 & $-30: 41: 55.00$ \\
\hline \multirow[t]{2}{*}{ RXCJ2218.6-3853 } & 06-17 June 2007 & 390 & 1.4 & 128 & EW352 & $22: 18: 40.20$ & $-38: 53: 51.00$ \\
\hline & 26,31 July 2007 & 201 & 1.4 & 128 & $750 \mathrm{~B}$ & $22: 18: 40.20$ & $-38: 53: 51.00$ \\
\hline \multirow[t]{2}{*}{ RXCJ2234.5-3744 } & 06-17 June 2007 & 407 & 1.4 & 128 & EW352 & $22: 34: 31.00$ & $-37: 44: 06.00$ \\
\hline & 26-31 July 2007 & 240 & 1.4 & 128 & $750 \mathrm{~B}$ & $22: 34: 31.00$ & $-37: 44: 06.00$ \\
\hline
\end{tabular}

Table 4.2: Details of $1.4 \mathrm{GHz}$ low-resolution pre-CABB images of our observed REXCESS cluster sub-sample.

\begin{tabular}{lccc}
\hline \hline $\begin{array}{l}\text { Cluster } \\
\text { Name }\end{array}$ & $\begin{array}{c}\text { Beam, PA } \\
\text { " } \times \text { " }{ }^{\circ}\end{array}$ & $\begin{array}{c}\sigma_{r m s} \\
\mu \text { Jy }_{\text {beam }}{ }^{-1}\end{array}$ & Comment \\
\hline RXCJ0006.0-3443 & $59 \times 35,+35$ & 116 & Pre-CABB \\
RXCJ0049.4-2931 & $71 \times 47,-9$ & 313 & Pre-CABB \\
RXCJ0145.0-5300 & $78 \times 34,-21$ & 200 & Pre-CABB \\
RXCJ0211.4-4017 & $70 \times 52,-10$ & 200 & Pre-CABB \\
RXCJ0225.1-2928 & $66 \times 46,+2$ & 124 & Pre-CABB \\
RXCJ0345.7-4112 & $66 \times 34,-13$ & 120 & Pre-CABB \\
RXCJ0616.8-4748 & $50 \times 46,-84$ & 147 & Pre-CABB \\
RXCJ0645.4-5413 & $67 \times 40,-62$ & 120 & Pre-CABB \\
RXCJ1236.7-3354 & $55 \times 48,-34$ & 120 & Pre-CABB \\
RXCJ2014.8-2430 & $55 \times 48,-33$ & 130 & Pre-CABB \\
RXCJ2023.0-2056 & $65 \times 50,-11$ & 228 & Pre-CABB \\
RXCJ2129.8-5048 & $61 \times 43,-59$ & 236 & Pre-CABB \\
RXCJ2149.1-3041 & $55 \times 48,-33$ & 240 & Pre-CABB \\
RXCJ2218.6-3853 & $39 \times 31,-39$ & 150 & Pre-CABB \\
RXCJ2234.5-3744 & $65 \times 46,-37$ & 135 & Pre-CABB \\
\hline
\end{tabular}

sented in Figure 4.2. Details of all the pre-CABB low resolution images at $1.4 \mathrm{GHz}$ are given in Table 4.2. 
Table 4.3: Details of new CABB ATCA observations of the three clusters with diffuse source candidates.

\begin{tabular}{|c|c|c|c|c|c|c|}
\hline Cluster & $\begin{array}{c}\text { Obs Dates } \\
\text { CABB }\end{array}$ & $\begin{array}{l}\text { Int Time } \\
\text { Mins }\end{array}$ & $\begin{array}{l}\text { Freq } \\
\mathrm{GHz}\end{array}$ & $\begin{array}{c}\text { Bandwidth } \\
\text { GHz }\end{array}$ & Config & $\begin{array}{l}\text { Pointing } \\
\text { RA, Dec }\end{array}$ \\
\hline \multirow[t]{3}{*}{ RXCJ0145.0-5300 } & 17 July 2011 & 135 & 2.1 & 2 & $\mathrm{H} 214$ & $01: 45: 02.30-53: 00: 50.00$ \\
\hline & 30 Dec 2011 & 1092 & 2.1 & 2 & $6 \mathrm{~A}$ & 01:45:02.30 -53:00:50.00 \\
\hline & 23,24 Mar 2012 & 280 & 2.1 & 2 & H168 & $01: 45: 02.30-53: 00: 50.00$ \\
\hline \multirow[t]{3}{*}{ RXCJ2129.8-5048 } & 16,17 July 2011 & 233 & 2.1 & 2 & $\mathrm{H} 214$ & $21: 29: 30.00-50: 48: 51.00$ \\
\hline & 28,29 Dec 2011 & 1003 & 2.1 & 2 & $6 \mathrm{~A}$ & $21: 29: 51.00-50: 48: 04.00$ \\
\hline & 23,24 Mar 2012 & 220 & 2.1 & 2 & H168 & $21: 29: 51.00-50: 48: 04.00$ \\
\hline \multirow[t]{3}{*}{ RXCJ2234.5-3744 } & 16,17 July 2011 & 260 & 2.1 & 2 & $\mathrm{H} 214$ & $22: 34: 23.00-37: 43: 29.00$ \\
\hline & 28,29 Dec 2011 & 1020 & 2.1 & 2 & $6 \mathrm{~A}$ & $22: 34: 31.00-37: 44: 06.00$ \\
\hline & 23,24 Mar 2012 & 273 & 2.1 & 2 & H168 & $22: 34: 31.00-37: 44: 06.00$ \\
\hline
\end{tabular}

Table 4.4: Details of $1.4 \mathrm{GHz}$ low resolution CABB + pre-CABB images of the clusters with diffuse candidates in our REXCESS cluster sub-sample.

\begin{tabular}{lcccc}
\hline \hline $\begin{array}{l}\text { Cluster } \\
\text { Name }\end{array}$ & $\begin{array}{c}\text { Beam, PA } \\
" \times^{\prime \prime},{ }^{\circ}\end{array}$ & $\begin{array}{c}\sigma_{r m s} \\
\mu \mathrm{Jy}_{\text {beam }}{ }^{-1}\end{array}$ & $\begin{array}{c}\text { Bandwidth } \\
\mathrm{MHz}\end{array}$ & Comment \\
\hline RXCJ0145.0-5300 & $84 \times 51,-28$ & 176 & 300 & CABB (Jul+Mar) + pre-CABB \\
RXCJ2129.8-5048 & $74 \times 52,-63$ & 200 & 300 & CABB (Jul+Mar) + Pre-CABB \\
RXCJ2234.5-3744 & $73 \times 58,-47$ & 120 & 300 & CABB (Jul+Mar) + pre-CABB \\
\hline
\end{tabular}

\section{$\S 4.4$. CABB Observations}

As mentioned earlier there were three clusters with diffuse emission candidates. We re-observed these three clusters to shed more light on the structures of the candidate sources. The ATCA follow-up observations in July and late December 2011 and March 2012 with $\mathrm{CABB}$ were performed in the H214, 6A and H168 configurations at the central frequency of $2.1 \mathrm{GHz}$ with a $2 \mathrm{GHz}$ bandwidth. These new CABB observations allowed us to probe various scales of three candidate clusters while this would enhance the uv and hour-angle coverage. Details of the new CABB radio observations of three of our REXCESS cluster sub-sample are presented in Table 4.3.

Different array configurations with a minimum baseline length of $31 \mathrm{~m}$ and maximum baseline of $\sim 6 \mathrm{~km}$ provides the angular sensitivity to the scales between $9^{\prime \prime}$ to $24^{\prime}$ at $1.4 \mathrm{GHz}$. Additionally, the highest angular resolution of $\sim 4^{\prime \prime}$ was achievable at 3.1 GHz.

All the data reduction strategies for the $\mathrm{CABB}$ data are given in Chapter 3 . For all the clusters with diffuse candidates we have combined available pre-CABB data with a 300 $\mathrm{MHz}$ sub-band $\mathrm{CABB}$ data to create new low resolution images. Investigation of these new low resolution images in conjunction with the high resolution images of the clusters 
Table 4.5: Details of the high resolution radio images of the REXCESS sub-sample at $1.4 \mathrm{GHz}$.

\begin{tabular}{|c|c|c|c|c|}
\hline Cluster & $\begin{array}{l}\text { Noise Level } \\
\mu \mathrm{Jy} \mathrm{beam}^{-1}\end{array}$ & $\begin{array}{c}\text { Beam,PA } \\
" \times^{\prime \prime}, \circ\end{array}$ & $\begin{array}{l}\text { Bandwidth } \\
\mathrm{MHz}\end{array}$ & Notes \\
\hline RXCJ0006.0-3443 & 105 & $14 \times 8,-6$ & 185 & pre-CABB \\
\hline RXCJ0049.4-2931 & 108 & $11 \times 4,-9$ & 185 & pre-CABB \\
\hline RXCJ0211.4-4017 & 120 & $8 \times 4,-10$ & 185 & pre-CABB \\
\hline RXCJ0225.1-2928 & 83 & $10 \times 4,-2$ & 185 & pre-CABB \\
\hline RXCJ0345.7-4112 & 90 & $10 \times 11,-21$ & 185 & pre-CABB \\
\hline RXCJ0616.8-4748 & 75 & $6 \times 5,-70$ & 185 & pre-CABB \\
\hline RXCJ0645.4-5413 & 80 & $7 \times 4,-70$ & 185 & pre-CABB \\
\hline RXCJ1236.7-3354 & 77 & $10 \times 4,13$ & 185 & pre-CABB \\
\hline RXCJ2014.8-2430 & 90 & $11 \times 4,-6$ & 185 & pre-CABB \\
\hline RXCJ2023.0-2056 & 125 & $19 \times 5,-6$ & 185 & pre-CABB \\
\hline RXCJ2149.1-3041 & 112 & $9 \times 4,-5$ & 185 & pre-CABB \\
\hline RXCJ2218.6-3853 & 63 & $8 \times 4,-2$ & 185 & pre-CABB \\
\hline Cluster & $\begin{array}{l}\text { Noise Level } \\
\mu \text { Jy beam }^{-1}\end{array}$ & $\begin{array}{c}\text { Beam,PA } \\
" \times{ }^{\prime \prime}, \circ\end{array}$ & $\begin{array}{l}\text { Bandwidth } \\
\mathrm{MHz}\end{array}$ & Notes \\
\hline RXCJ0145.0-5300 & 60 & $6 \times 4,-12$ & 300 & $\mathrm{CABB}, 6 \mathrm{~A}$ \\
\hline RXCJ2129.8-5048 & 105 & $8 \times 4,10$ & 300 & $\mathrm{CABB}, 6 \mathrm{~A}$ \\
\hline RXCJ2234.5-3744 & 105 & $8 \times 7,7$ & 300 & $\mathrm{CABB}, 6 \mathrm{~A}$ \\
\hline
\end{tabular}

with candidate diffuse emissions confirmed the existence of a giant radio halo in Abell 3888 , however the two remaining radio relic candidates turned to be a head-tail galaxy and a bright extended source in clusters RXCJ2129.8-5048 (A3771) and RXCJ0145.05300, respectively. The confirmed radio halo in A3888 and the discovered HT galaxy in A3771 are presented later in Chapters 5 and 8, respectively. Details of the $1.4 \mathrm{GHz}$ low resolution images made of $\mathrm{CABB}(\mathrm{H} 168+\mathrm{H} 214)+$ pre-CABB are given in Table 4.4.

\section{$\S 4.5$. High Resolution Images}

All the data reduction procedures of the pre-CABB ATCA are given previously in $\S 4.3$. Our practical data reduction and imaging strategies for the $\mathrm{CABB}$ data are explained earlier in Chapter 3. The entire band is sub-divided into 2048 channels where each channel has $\approx 1 \mathrm{MHz}$ width. All RFI removal and calibration routines were repeated using the MIRIAD package. Details of radio observations of our REXCESS sub-sample are given in Table 4.5. We have made the full resolution Stokes I images without any tapering applied and the parameter, "robust" in the task "invert" in Miriad software (Sault et al., 1995) was set to 0 and 0.5 to use different weighting schemes. Details of the high resolution images are given in Table 4.5.

Examining the final restored Stokes I high resolution images revealed a number of interesting extended sources. In each section, global properties of one of our observed clusters, the total number of detected radio sources and their classifications are provided.

Each section has a plot displaying the ATCA primary beam ${ }^{1}$ with a blue circle and

\footnotetext{
${ }^{1}$ The primary beam is defined as the half power point on the first maxima of the airy disk pattern for a single dish. It is effectively equivalent to the area over which reliable flux measurements can be made.
} 
a large red circle with a $2 \mathrm{Mpc}$ radius around a cluster. Positions of detected radio sources in the field at $1.4 \mathrm{GHz}$ are also annotated with open red circles. To avoid an over crowded plot, only the radio IDs of extended sources are annotated on the background radio image but the full list of radio IDs and their properties can be found in the Appendices. In each plot green contours show the X-ray emission of the cluster observed with the XMM-Newton X-ray telescope (Pratt et al., 2009). Postage stamps of detected extended radio sources in each cluster are also provided. In all postage stamps the green contour represents the Sydney University Molonglo Sky Survey (SUMSS; Mauch et al., 2003) at $843 \mathrm{MHz}$ for the clusters with $\delta<-30^{\circ}$ and for clusters with $\delta>$ $-30^{\circ}$ the green contours represent the NRAO VLA Sky Survey (NVSS, Condon et al., 1998) radio emission at $1.4 \mathrm{GHz}$. The beam size of the NVSS image is $45^{\prime \prime} \times 45^{\prime \prime}$ and for the SUMSS image a beam size is $43^{\prime \prime} \times 43^{\prime \prime} \operatorname{cosec}(|\operatorname{Dec}|)$.

\section{$\S$ 4.6. Source Detection}

Duchamp, a publicly available source finder software (Whiting, 2012), was used for source detection in all high resolution images at $1.4 \mathrm{GHz}$. In the Duchamp input parameter list, we set the snrCut value to 5 to avoid false positive detections, particularly at the edges of the image where the noise was significantly higher. In a few cases an extremely bright source was in the field and the region around that source could not be properly CLEANed. Hence, the area around that bright source had higher local noise level, thus $s n r C u t$ was set to 6 or 7 to avoid false detections. However due to using higher snrCut value some radio objects in regions around that bright source remained undetected. To overcome this issue, sub-images only containing the non-detected objects were used as an input fits file for Duchamp to avoid false non-detection. To avoid under estimating the flux of the detected source we used the growing option in the Duchamp and we set the parameter growthCut to 3 .

We have inspected the all detected sources within the primary beam and classified them according to their morphology. For the purpose of radio source classification, we have used numbers 1 to 6 where $1=$ Point source, $2=$ Bent-tail radio source (BT) candidate, 3 = Head-tail radio source $(\mathrm{HT})$ candidate, $4=$ Wide Angle Tail (WAT) candidate, $5=$ Double radio source candidate, $6=$ Extended radio source which can not be classified.

In the case that a redshift was available, the k-corrected radio power for each source was calculated using the equation given in 5.1.

For the clusters with available SUMSS observations, the integrated spectral index of detected sources were determined and used in the power calculation. However, for many It is in some senses equivalent to the field of view. 
detected radio sources in our radio images, the spectral index could not be determined since the SUMSS image was not available. Additionally, in some cases, due to the lower resolution of SUMSS images, two or more radio sources detected in our high resolution maps were blended into one radio source in the SUMSS image. Moreover, some of our detections had no corresponding emission at $843 \mathrm{MHz}$ due to local insensitivity of the SUMSS image. In some cases, despite the fact that the source was detected in the SUMSS image, the flux measurements at $843 \mathrm{MHz}$ was not feasible due to flux scaling issue in the SUMSS image where the total flux was less than the peak brightness. We also noted that the source was detected in the SUMSS image, however, the SUMSS image suffered artefacts or the source was surrounded by negative bowl which made the flux measurements at $843 \mathrm{MHz}$ impossible. In all aforementioned cases, the typical spectral index ${ }^{2}$ for radio galaxies, $\alpha=-0.8$ was adopted (Condon, 1992) in the power calculations.

In the following sections, the details of the detected radio objects at $1.4 \mathrm{GHz}$ for each observed cluster are given along with the description of the cluster.

\section{§4.7. REXCESS Cluster RXCJ0006.0-3443}

The X-ray emission of cluster RXCJ0006.0-3443 (A2127) is slightly asymmetric and there is no radio emission associated with central cD galaxy. In this case, the high resolution radio image had a beam size of $14^{\prime \prime} \times 8^{\prime \prime}$, $\mathrm{pa}=-6^{\circ}$ and $\sigma_{r m s}=105 \mu \mathrm{Jy}_{\text {beam }}{ }^{-1}$. Examining the total brightness image revealed that there are 33 radio sources detected in the primary beam of the ATCA at $1.4 \mathrm{GHz}$ ( Figure 4.3). There are 17 radio sources within a $2 \mathrm{Mpc}$ radius around the cluster. Details of the detected radio sources in the primary beam of the ATCA and their properties are given in Appendix A.1.

This cluster has an angular scale of $1 \mathrm{Mpc}=7.69^{\prime}$ at $\mathrm{z}=0.1147$ (Haarsma et al., 2010). Five radio sources had optical IDs but only one optical counterpart had a measured redshift from the literature. Radio source 26 with redshift of $\mathrm{z}=0.1947$ (Colless et al., 2003) is likely to be a background galaxy as the redshift difference between this source and the cluster is significant. Hence, Source 26 is not a cluster member.

Eight extended radio sources are detected in the ATCA field. In Figure 4.4 the radio contours of the detected extended sources are shown in red, overlaid on the DSS blue image. Green contours of the radio emission in the SUMSS image are also overlaid.

Radio Source 02 is a BT radio source with an optical counterpart. Radio source 03 is an HT candidate with an optical ID and source 04 is a complex extended radio source. The radio contours suggest that it could be an X-shape radio galaxy but enhanced resolution is required to confirm the structure of the source. Radio sources 06

\footnotetext{
${ }^{2}$ The convention of $\mathrm{s} \propto v^{\alpha}$ is assumed.
} 
and 15 is extended radio sources with no accurate classification. Source 07 is an HT candidate without an optical ID. Sources 23 is an extended source but the structures are not clear. Source 29 is an extended diffuse source with no optical counterpart. There are no core or hot spots associated with this source in the high resolution image created only including baselines greater than $3000 \mathrm{~m}$ (antenna 6 only). This might be a dying radio galaxy at higher redshift, however, classification of this source is not feasible due to lack of sufficient data.

\section{§4.8. REXCESS Cluster RXCJ0049.4-2931}

RXCJ0049.4-2931 (Abell S0084) is a regular cluster with symmetric X-ray emission (Weißmann et al., 2013) with no cD galaxy in the centre. The angular scale of the cluster is $1 \mathrm{Mpc}=8.06^{\prime}$ at $\mathrm{z}=0.1084$ (Haarsma et al., 2010). The high resolution radio image of the cluster has a beam size of $11^{\prime \prime} \times 4^{\prime \prime}, \mathrm{pa}=-9^{\circ}$ and $\sigma_{r m s}=108 \mu \mathrm{Jy} \mathrm{beam}^{-1}$ at $1.4 \mathrm{GHz}$. There are 18 radio sources detected in the primary beam of the ATCA at $1.4 \mathrm{GHz}$. There are 10 radio sources within a $2 \mathrm{Mpc}$ radius around the cluster. In Figure 4.5 all the detected radio sources are shown with open red circles and the radio IDs of extended radio sources are also annotated on the full resolution radio image. Green contours represent the X-ray emission of the cluster. Details and the properties of all detected radio sources in the primary beam of the ATCA are given in Appendix A.2.

Of the four sources with optical IDs, three have redshift measurements available in the literature. The radio source 03 with redshift of 0.1104 (Jones et al., 2009) has an angular separation of $14.53^{\prime}(1.8 \mathrm{Mpc})$ from the cluster centre. This object is likely a cluster member at the outskirts of the cluster. Radio object 01 with redshift of 0.0320 (Croom et al., 2001) and source 02 with redshift of 0.0767 (Collins et al., 1995) are not part of the cluster. In Figure 4.6, radio contours of extended radio sources are overlaid on DSS blue image. Radio source 07 is an extended sources which could not be classified due to the lack of resolution. Source 15 is a double radio source candidate.

\section{§4.9. REXCESS Cluster RXCJ0145.0-5300}

Cluster RXCJ0145.0-5300 (A2941) has no central cD galaxy and posses multiple BCGs in the centre. The X-ray emission of the cluster is elongated. The angular scale of the cluster is $1 \mathrm{Mpc}=7.56^{\prime}$ at $\mathrm{z}=0.1168$ (Haarsma et al., 2010). The high resolution radio image of the cluster has a beam size of $6^{\prime \prime} \times 4^{\prime \prime}$ with the pa $=-12^{\circ}$ and $\sigma_{r m s}=$ $60 \mu \mathrm{Jy}_{\text {beam }}{ }^{-1}$ at $1.4 \mathrm{GHz}$. A total of 28 radio sources are in the primary beam of the ATCA at $1.4 \mathrm{GHz}$. There are 23 radio sources within a $2 \mathrm{Mpc}$ radius around the cluster. Figure 4.7 shows the X-ray emission of the cluster overlaid on the radio image (1.4 
$\mathrm{GHz}$ ). All the details and properties of the detected radio sources in the primary beam of the ATCA at $1.4 \mathrm{GHz}$ are given in Appendix Table A.3. There is a very bright point source on the leftmost of the map which appears as an extended source. This is because the CLEANing of the source has left some artefacts around the source. There are nine radio sources with optical counterparts but only one optical ID has a measured redshift from the literature. Source 15 has an optical counterpart with $\mathrm{z}=0.1182$ (Guzzo et al., 2009) and resides in the core of the cluster, hence it is highly likely a cluster member.

There are six extended radio sources in the ATCA primary beam. In Figure 4.8, radio contours of the detected extended sources in the primary beam of the ATCA are shown on the DSS blue image along with the SUMSS data in green. Radio object 01 is an HT radio source and object 02 is a BT candidate. Source 03 is also a BT candidate and source 05 is an HT radio source. Structure of source 08 is not clear due to lack of resolution but appears to have a significant diffuse component. Source 24 is an extended radio source with a jet of unclear classification. There are 24 radio sources within a 2 $\mathrm{Mpc}$ radius around the cluster.

\section{$\S$ 4.10. REXCESS Cluster RXCJ0211.4-4017}

Cluster RXCJ0211.4-4017 (A2948) has a central cD galaxy with radio mission. The Xray emission of the cluster is symmetric and Weißmann et al. (2013) has reported this is a regular cluster. The angular scale of the cluster is $1 \mathrm{Mpc}=8.6^{\prime}$ at $\mathrm{z}=0.1008$ (Haarsma et al., 2010). The high resolution image of the cluster has a beam size of $8^{\prime \prime} \times 4^{\prime \prime}$, pa $=$ $-10^{\circ}$ and $\sigma_{r m s}$ is $120 \mu \mathrm{Jy}_{\text {beam }}{ }^{-1}$ at $1.4 \mathrm{GHz}$. There are 16 radio sources detected in the primary beam of of the ATCA at $1.4 \mathrm{GHz}$. There are 10 radio sources within a $2 \mathrm{Mpc}$ radius around the cluster (Figure 4.9). Figure 4.10 shows the $\mathrm{cD}$ galaxy (source 6) and the other extended sources in the field, source 16. All the details of the detected radio sources and their properties are given in Appendix Table A.4.

There are three radio sources with optical counterparts and one of them has measured redshift available from the literature. Radio source 6 , the $\mathrm{cD}$ galaxy of the cluster, has an optical ID with redshift of 0.105 (Grazian et al., 2002) and is classified as a double radio galaxy. Source 16 is a classic WAT with an optical counterpart.

\section{$\S$ 4.11. REXCESS Cluster RXCJ0225.1-2928}

Cluster RXCJ0225.1-2928 with redshift of 0.0604 (Haarsma et al., 2010) has a central cD galaxy with no radio emission. The angular scale of the cluster is $1 \mathrm{Mpc}=13.8^{\prime}$. The X-ray emission of the cluster is slightly elongated towards the East and Weißmann et al. (2013) pointed out this is an intermediate cluster. The high resolution image of 
the cluster has a beam size of $10^{\prime \prime} \times 4^{\prime \prime}, \mathrm{pa}=-2^{\circ}$ and $\sigma_{r m s}=115 \mu \mathrm{Jy}$ beam $^{-1}$ at the 1.4 $\mathrm{GHz}$. There are 20 radio sources found in the primary beam of the ATCA at $1.4 \mathrm{GHz}$ (Figure 4.11) and all of them reside within a $2 \mathrm{Mpc}$ radius around the cluster. Details of the detected radio sources and their properties are presented in Appendix Table A.5.

There is only one radio object with optical counterpart (source 18) which has no redshift measurement available in the literature. There are two extended radio sources in the primary beam of the ATCA (Figure 4.12). Radio source 04 is an HT candidate, Source 17 is an extended radio source which is slightly elongated north-south. Radio object 20 is a WAT candidate with no optical counterpart. Radio object 15 is an extended diffuse radio emission candidate. Detailed investigation of this source is given in $\S 5.2$.

\subsection{REXCESS Cluster RXCJ0345.7-4112}

Cluster RXCJ0345.7-4112 (S0384) has a central cD galaxy with extended radio emission. The X-ray emission of the cluster is symmetric and Weißmann et al. (2013) have classified it as a regular cluster. The angular scale of the cluster is $1 \mathrm{Mpc}=13.71^{\prime}$ at redshift of the cluster. The high resolution image of the cluster has a beam size of $10^{\prime \prime} \times$ $11^{\prime \prime}, \mathrm{pa}=-21^{\circ}$ and $\sigma_{r m s}=90 \mu \mathrm{Jy}_{\text {beam }}{ }^{-1}$ at $1.4 \mathrm{GHz}$. In this case, the majority of extended mission are over resolved using robust $=0$ or 0.5 , thus, here we used robust $=$ 1 instead to construct the high resolution image. There are 29 radio sources detected in the primary beam of the ATCA at $1.4 \mathrm{GHz}$ and all are within a $2 \mathrm{Mpc}$ radius around the cluster (Figure 4.13). Details of all detected radio sources and their properties are given in Appendix Table A.6.

Nine radio sources had optical counterparts, of which two had confirmed redshifts in the literature. Radio source 16 with redshift 0.06 (Muriel et al., 1995) is the cD galaxy of the cluster. Source 19 with redshift of 0.079 (Pimbblet et al., 2006) which is $15.78^{\prime}$ $(1.15 \mathrm{Mpc})$ separated from the cluster centre could also be a cluster member.

There are eight extended radio sources in the primary beam of the ATCA (Figure 4.14) Radio source 01 is an extended source which can not be classified. Source 02 and 07 are BT candidates. radio object 09 is an HT candidate. Radio source 10 is a WAT candidate with an optical counterpart. Source 16 is a double radio source. source 18 can be an HT candidate. Radio source 29 has extended radio emission but further classification is not possible due to lack of sufficient resolution. There are 15 radio sources within a $2 \mathrm{Mpc}$ radius around the cluster. 


\section{\$4.13. REXCESS Cluster RXCJ0616.8-4748}

Cluster RXCJ0616.8-4748 has a central cD galaxy with no radio emission. The Xray emission of the cluster is reasonably symmetric and this cluster is classified as an intermediate cluster (Weißmann et al., 2013). The angular scale of the cluster is $1 \mathrm{Mpc}$ $=7.58^{\prime}$ at $\mathrm{z}=0.1164$ (Haarsma et al., 2010). The high resolution image of the cluster has a beam size of $6^{\prime \prime} \times 5^{\prime \prime}, \mathrm{pa}=-70^{\circ}$ and $\sigma_{r m s}=75 \mu \mathrm{Jy}_{\text {beam }}{ }^{-1}$ at $1.4 \mathrm{GHz}$. This cluster had 31 radio sources in the primary beam of the ATCA at $1.4 \mathrm{GHz}$ (Figure 4.15). There are 15 radio sources within a $2 \mathrm{Mpc}$ radius around the cluster. Details of all detected radio sources in the primary beam of the ATCA at $1.4 \mathrm{GHz}$ are given in Appendix Table A.7.

There are six radio objects with optical counterparts but only one had a measured redshift in the literature. Radio source 07 has an optical ID with $\mathrm{z}=0.044$ (Huchra et al., 2012) which is considerably less than the cluster redshift thus this object is not a cluster member.

There are five extended radio sources in the primary beam of the ATCA (Figure 4.16). Sources $03,10,16$ and 26 are double radio galaxies. Sources 05 and 24 are complex extended sources with no classification. Source 24 is very diffuse.

\section{$\S$ 4.14. REXCESS Cluster RXCJ0645.4-5413}

The cluster RXCJ0645.4-5413 (A3404) has the highest redshift in our REXCESS subsample with $\mathrm{z}=0.1644$ (Haarsma et al., 2010). The angular scale of the cluster is 1 $\mathrm{Mpc}=5.67^{\prime}$ at this redshift. This cluster has no central $\mathrm{cD}$ galaxy but multiple BCGs are present in the core of the cluster. The X-ray emission of the cluster is elongated towards the north-west and this an intermediate cluster (Weißmann et al., 2013). The high resolution image of the cluster has a beam size of $7^{\prime \prime} \times 4^{\prime \prime}$, pa $=-70^{\circ}$ and $\sigma_{r m s}=$ $80 \mu \mathrm{Jy}$ beam $^{-1}$ at $1.4 \mathrm{GHz}$. This cluster has 16 radio sources detected in the primary beam of the ATCA at $1.4 \mathrm{GHz}$ (Figure 4.17). There are 7 radio sources within a $2 \mathrm{Mpc}$ radius around the cluster. Details of the detected radio objects in the primary beam of the ATCA at $1.4 \mathrm{GHz}$ are given in Appendix Table A.8.

There are three radio objects with the optical counterparts but none of them had available redshift in the literature. There are nine extended radio objects detected at $1.4 \mathrm{GHz}$ (Figure 4.18). Radio objects 01 and 05 are complex radio sources and the classification is not possible due to lack of resolution. Radio object 08 is a double radio source with no optical counterpart. Object 09 is an HT candidate, however there is an offset between the ATCA and the SUMSS images. Radio source 11 is an HT candidate with an optical counterpart. Radio source 12 is an extended radio emission which can not be classified. Radio object 14, 15, and 16 are complex objects and can 
not be classified. Object 14 has a diffuse component around it and Source 16 has a very odd diffuse component and part of the object appears as a point source with an optical counterpart.

\subsection{REXCESS Cluster RXCJ1236.7-3354}

The cluster RXCJ1236.7-3354 (A0700) has no central cD galaxy but a BCG is present in the core of the cluster. The X-ray emission of the cluster is symmetric and Weißmann et al. (2013) reported this is an intermediate cluster. The angular scale of the cluster is $1 \mathrm{Mpc}=10.63^{\prime}$ at $\mathrm{z}=0.0796$ (Haarsma et al., 2010). The high resolution image has a beam size of $10^{\prime \prime} \times 4^{\prime \prime}$, $\mathrm{pa}=13^{\circ}$ and $\sigma_{r m s}=77 \mu \mathrm{Jy} \mathrm{beam}^{-1}$. This cluster has 40 radio sources detected in the primary beam of the ATCA at $1.4 \mathrm{GHz}$ (Figure 4.19). There are 39 radio sources within a $2 \mathrm{Mpc}$ radius around the cluster. In Appendix Table A.9 all the details of the detected radio sources in the primary beam of the ATCA at $1.4 \mathrm{GHz}$ are given.

There are seven radio objects with the optical counterparts of which three had available redshifts in the literature. Object 25 is the BCG of the cluster with $\mathrm{z}=0.0806$ (Jones et al., 2009). Radio object 08 with $\mathrm{z}=0.0850$ (Jones et al., 2009) is $11.58^{\prime}$ ( $1 \mathrm{Mpc}$ ) separated from the cluster core and is likely a cluster member. Radio object 12 with $\mathrm{z}=0.0817$ (Jones et al., 2009) is located at $8.5^{\prime}$ (800 kpc)away from the cluster and is probably a cluster member.

There are seven extended radio sources in the primary beam of the ATCA at $1.4 \mathrm{GHz}$ (Figure 4.20). The cluster RXCJ1236.7-3354 is the only cluster which has available TIFR GMRT Sky Survey (TGSS) image at $150 \mathrm{MHz}$. Radio source 04 is a double radio source with an optical counterpart. Radio source 07 is a complex extended radio source with no classification. Radio object 14 is a double radio source and a point source or maybe a single source with jets. Radio object 25 is a WAT candidate with optical ID which has been partially detected in the TGSS image at $150 \mathrm{MHz}$. We measured spectral indices of the top and bottom lobes as -2.23 and -2.03 , respectively using 1.4 GHz ATCA and SUMSS $843 \mathrm{MHz}$ fluxes. There is a partial offset from the ATCA and SUMSS+TGSS images. Radio sources 33 and 35 can be the components of one source but due to lack of sensitivity accurate classification is not possible. Radio object 39 is a double radio source with an optical counterpart.

\section{$\S$ 4.16. REXCESS Cluster RXCJ2014.8-2430}

The cluster RXCJ2014.8-2430 with a central cD galaxy and $\mathrm{z}=0.1612$ (Böhringer et al., 2004) $\left(1 \mathrm{Mpc}=5.8^{\prime}\right)($ Haarsma et al., 2010) has symmetric X-ray emission. Weißmann 
et al. (2013) reported that this is a regular cluster. The high resolution image has a beam size of $11^{\prime \prime} \times 4^{\prime \prime}$, pa $=-6^{\circ}$ and $\sigma_{r m s}=90 \mu \mathrm{Jy}$ beam $^{-1}$ at $1.4 \mathrm{GHz}$. This cluster has 17 radio sources detected in the primary beam of the ATCA at $1.4 \mathrm{GHz}$ (Figure 4.21). There are 4 radio sources within a $2 \mathrm{Mpc}$ radius around the cluster. All the details and properties of the detected radio sources in the primary beam of the ATCA at $1.4 \mathrm{GHz}$ are given in Appendix Table A.10.

There are three radio objects with optical counterparts, but only one had an available redshift in the literature. Radio object 06 with redshift of 0.157 (Jones et al., 2009) is the $\mathrm{cD}$ galaxy of the cluster.

There are five extended radio sources detected at $1.4 \mathrm{GHz}$ (Figure 4.22). Source 06 (cD galaxy) appears to be an extended source, however, this is an artefact due to CLEANing problem. The cD galaxy is a very bright point source with a flux of 216 mJy at $1.4 \mathrm{GHz}$.

Object 07 is an extended radio source which can not be classified due to lack of resolution. Radio source 08 is an HT candidate with no optical ID. Radio object 11 is a BT candidate with no optical counterpart. Radio source 12 is a double radio object and radio source 14 is a WAT candidate with an optical counterpart.

\section{§4.17. REXCESS Cluster RXCJ2023.0-2056}

The cluster RXCJ2023.0-2056 (S0868) has the lowest redshift in our REXCESS subsample. This cluster with central $\mathrm{cD}$ galaxy has a symmetric X-ray emission but Weißmann et al. (2013) reported that this is an intermediate cluster. The angular scale of the cluster is $1 \mathrm{Mpc}=14.59^{\prime}$ at $\mathrm{z}=0.0564$ (Haarsma et al., 2010).

The high resolution image of the cluster has a beam size of $19^{\prime \prime} \times 5^{\prime \prime}$, pa $=-6^{\circ}$ and $\sigma_{r m s}=145 \mu \mathrm{Jy}_{\text {beam }}{ }^{-1}$. This cluster has 17 radio sources detected in the primary beam of the ATCA at $1.4 \mathrm{GHz}$ and all of them reside within a $2 \mathrm{Mpc}$ radius around the cluster (Figure 4.23). All the details and properties of the detected radio objects can be found in Appendix Table A.11.

There are three radio objects with the optical counterparts but only one had a redshift in the literature. The radio source 10 has redshift of 0.0262 (Strauss et al., 1992) and it is located at $14.81^{\prime}(\sim 1 \mathrm{Mpc})$ from the cluster core. This radio source has a considerably lower redshift than the cluster and thus it is a foreground galaxy. There are four extended radio objects in the primary beam of the ATCA at 1.4 GHz (Figure 4.24). Radio sources 06 and 12 are extended radio sources but accurate classification is not possible. Extended radio object 07 lies at the core of the cluster and is a BT candidate. Radio object 13 is an HT candidate with no optical ID. 


\section{\$4.18. REXCESS Cluster RXCJ2129.8-5048}

The cluster RXCJ2129.8-5048 (A3771) with no central cD galaxy has the lowest X-ray luminosity in our sub-sample. The X-ray emission in this cluster is highly elongated and Weißmann et al. (2013) reported that this is a double cluster. The high resolution image has a beam size of $8^{\prime \prime} \times 4^{\prime \prime}$ and $\mathrm{pa}=10^{\circ}$ and $\sigma_{r m s}=105 \mu \mathrm{Jy}_{\text {beam }}{ }^{-1}$ at $1.4 \mathrm{GHz}$. This cluster has 24 radio sources detected (at $6 \times \sigma_{r m s}$ ) in the primary beam of the ATCA at $1.4 \mathrm{GHz}$ (Figure 4.25). There are 21 radio sources within a $2 \mathrm{Mpc}$ radius around the cluster. All the details and properties of the detected sources in the primary beam of the ATCA at $1.4 \mathrm{GHz}$ can be found in Appendix Table A.12.

The angular scale of the cluster is $1 \mathrm{Mpc}=10.63^{\prime}$ at $\mathrm{z}=0.0796$ (Haarsma et al., 2010). There are five radio objects with optical counterparts of which, three have available redshifts in the literature. Radio source 08 is an HT galaxy with redshift of 0.0799 (Corwin and Emerson, 1982) and it resides 4.51' (424 kpc) away from the cluster thus it is highly likely a cluster member. This object is studied in more detail in Chapter 8. Radio source 11 is a WAT in the core of the cluster with redshift of 0.07691 (Jones et al., 2009). Radio object 20 with redshift of 0.0751 (Schwope et al., 2000) is located at $17.43^{\prime}(1.63 \mathrm{Mpc})$ from the cluster and it is probably a cluster member in the outskirts of the cluster.

There are six extended radio objects in the field (Figure 4.26). Sources 08 and 11 are described earlier as an HT and WAT in the cluster, respectively. Radio object 10 is a double radio source with an optical ID. Radio object 15 is an HT candidate with an optical ID. Source 17 is a BT candidate and radio object 22 is an extended source which due to insufficient resolution accurate radio classification remains elusive.

\section{$\S$ 4.19. REXCESS Cluster RXCJ2149.1-3041}

The cluster RXCJ2149.1-3041 (A3814) with a central cD galaxy has symmetric X-ray emission and Weißmann et al. (2013) reported that this is a regular cluster. The angular scale of the cluster is $1 \mathrm{Mpc}=7.47^{\prime}$ at $\mathrm{z}=0.1184$ (Haarsma et al., 2010).

The high resolution image of the cluster has a beam size of $9^{\prime \prime} \times 4^{\prime \prime}, \mathrm{pa}=-5^{\circ}$ and $\sigma_{r m s}$ $=112 \mu \mathrm{Jy}_{\text {beam }^{-1}}$. This cluster has 37 radio sources detected in the primary beam of the ATCA at $1.4 \mathrm{GHz}$ (Figure 4.27). There are 18 radio sources within a $2 \mathrm{Mpc}$ radius around the cluster. All the details of the detected radio objects in the primary beam of the ATCA at $1.4 \mathrm{GHz}$ are given in Appendix Table A.13.

There are five radio objects with optical counterparts of which four had available redshifts in the literature. Radio source 04 with redshift of 0.0735 (Jones et al., 2009) is not a cluster member since there was a significant redshift difference between this 
source and the cluster. Source 17 with redshift of 0.102 (Jones et al., 2009) is located at $16.21^{\prime}(2.15 \mathrm{Mpc})$ is highly likely not a cluster member. Source 26 with redshift of 0.1193 (Jones et al., 2009) is $8.24^{\prime}$ or $1.1 \mathrm{Mpc}$ away from the cluster centre hence this source is probably a cluster member. Source 37 with redshift of 0.0365 (Jones et al., 2009 ) is not a cluster member on account of incredible redshift difference between this source and the cluster.

There are eight extended radio sources in the primary beam of the ATCA at 1.4 $\mathrm{GHz}$ (Figure 4.28). Radio source 10 is a double radio source with no optical ID. Radio sources 11 and 13 are extended radio sources but there is a possibility that both sources are the components of a BT radio source where the jets remained undetected. Radio object 16 is a double radio source. Radio object 25 shows extended emission with no optical ID. Radio object 31 is a double radio source with no optical counterpart. Radio object 35 is a double radio source candidate with no optical ID.

\subsection{REXCESS Cluster RXCJ2218.6-3853}

The cluster RXCJ2218.6-3853 (A3856) with a central cD galaxy has an elongated Xray emission abut Weißmann et al. (2013) reported this is a regular cluster. The angular scale of the cluster is $1 \mathrm{Mpc}=6.43^{\prime}$ at $\mathrm{z}=0.1411$ (Haarsma et al., 2010).

The high resolution image has a beam size of $8^{\prime \prime} \times 4^{\prime \prime}$, $\mathrm{pa}=-2^{\circ}$ and $\sigma_{r m s}=65 \mu \mathrm{Jy}$ beam $^{-1}$ at $1.4 \mathrm{GHz}$. This cluster has 34 radio sources detected in the primary beam of the ATCA at $1.4 \mathrm{GHz}$ (Figure 4.29). There are 12 radio sources within a $2 \mathrm{Mpc}$ radius around the cluster. All the detailed and properties of their detected radio sources in the primary beam of the ATCA at $1.4 \mathrm{GHz}$ are given in Appendix Table A.14.

There are five radio objects with the optical counterparts and one of them had an available redshift in the literature. Radio object 19 with redshift of 0.1423 (Jones et al., 2009 ) is the cluster's cD galaxy.

There are 12 extended sources in the primary beam of the ATCA at $1.4 \mathrm{GHz}$ (Figure 4.30). Source 01 is an extended source with no classification. Objects 02 and 03 are HT candidates with no optical IDs. Source 11 and 18 are double radio sources candidates. Radio object 19 can be an HT and radio object 21 is an extended source with no radio classification as enhanced resolution is required for accurate classification. Radio object 22 is a BT candidate with no optical ID. Radio object 24 is an HT with an optical ID. Radio source 25 is also an HT without any optical ID. Radio source 26 is an extended source with no classification. Source 30 is also an HT radio source with no optical counterpart. 


\subsection{REXCESS Cluster RXCJ2234.5-3744}

Cluster RXCJ2234.5-3744 (A3888) with multiple BCGs has symmetric X-ray emission and Weißmann et al. (2013) reported that this is an intermediate cluster. The angular scale of the cluster is $1 \mathrm{Mpc}=6.02^{\prime}$ at $\mathrm{z}=0.1529$ (Pimbblet et al., 2006).

The high resolution image has a beam size of $8^{\prime \prime} \times 7^{\prime \prime}, \mathrm{pa}=7^{\circ}$ and $\sigma_{r m s}=105 \mu \mathrm{Jy}$ beam $^{-1}$ at $1.4 \mathrm{GHz}$. This cluster had 33 radio sources detected in the primary beam of the ATCA at $1.4 \mathrm{GHz}$ (Figure 4.31). There are 17 radio sources within a $2 \mathrm{Mpc}$ radius around the cluster. All the details and properties of the detected radio sources in the primary beam of the ATCA at $1.4 \mathrm{GHz}$ are given in Appendix Table A.15.

There are six radio objects with the optical counterparts and five of them had available redshifts in the literature. Radio source 09 with redshift of 0.284 (Pimbblet et al., 2006) is a background source. The radio object 11 with redshift of 0.1523 (Pimbblet et al., 2006) is located $3.18^{\prime}$ ( $528 \mathrm{kpc}$ ) away from the cluster centre and it is highly likely the cluster member. The radio object 12 with redshift of 0.1514 (Pimbblet et al., 2006) resides at $5.28^{\prime}(877 \mathrm{kpc})$ away from the cluster and it is also highly likely the cluster member. The radio object 16 with redshift of 0.1672 (Jones et al., 2009) is 9.68' (1.6 $\mathrm{Mpc}$ ) far from the cluster core and probably is not a cluster member. Radio object 17 with redshift of 0.1525 (Teague et al., 1990) is one of the BCGs in the cluster.

There are eight extended radio sources in the primary beam of the ATCA (Figure 4.32). Radio objects and 14 are double radio sources with no optical ID. Radio source 09 is a background double radio source with Objects 11,12,15 and 17 are all HT galaxies associated with the cluster. Source 17 has two components which could each be an HT galaxy but due to lack of enough resolution they are not resolved at $1.4 \mathrm{GHz}$ image. This is an exceptionally large number of HTs to be in one cluster and supports that there must be ongoing dynamical interactions in the cluster. Radio object 27 is a HT candidate with no optical ID, likely not associated with A3888.

\section{$\S$ 4.22. Summary}

In this chapter we presented details of pre-observed pre-CABB observations of our REXCESS sub sample. In the $1.4 \mathrm{GHz}$ pre-CABB low resolution images we found three diffuse candidates of which one was a radio halo candidate in RXCJ2234.5-3744 (A3888) and the two remaining were radio relic candidates in the clusters RXCJ0145.05300 and RXCJ2129.8-5048, respectively. We re-observed these clusters with CABB in July and December 2011 (H214 and 6A, respectively) to verify our findings. After data reduction and imaging we could confirm a radio halo in A3888 which will be presented in the next chapter. however, the other radio relic candidates were in fact a HT galaxy 

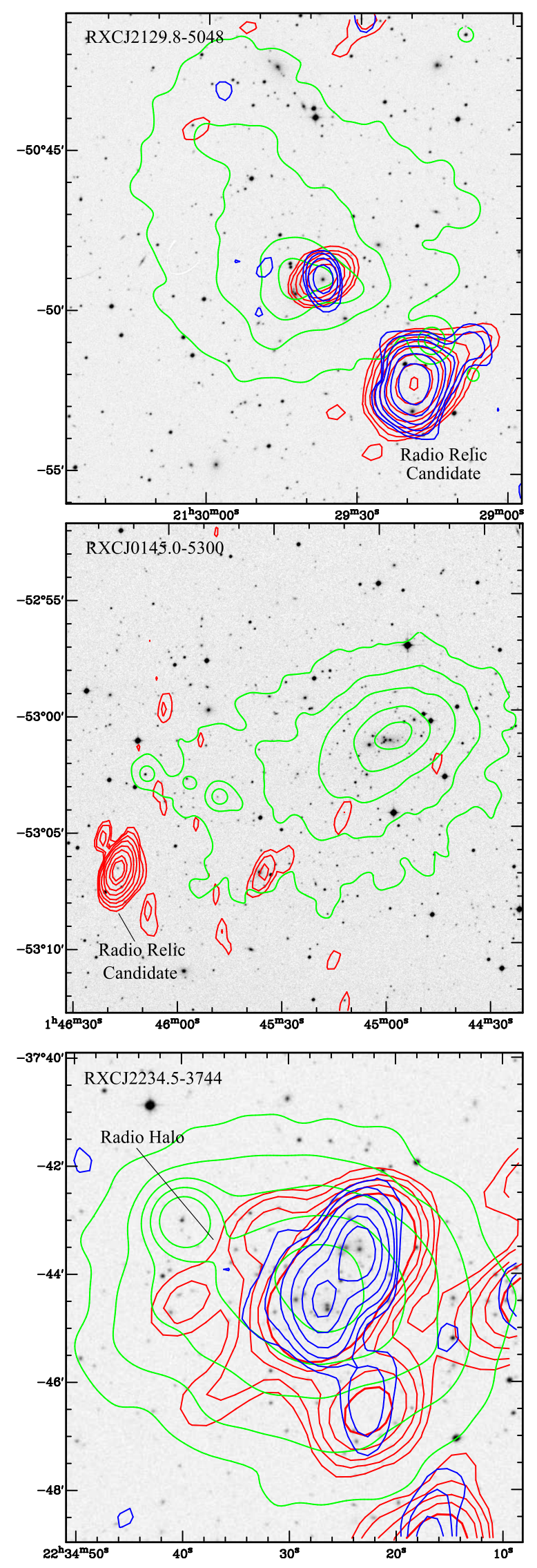

Figure 4.2: Low resolution 1.4 GHz pre-CABB images of three clusters with candidate diffuse sources. Red contours represent the radio emission at $1.4 \mathrm{GHz}$. The red contour levels are $\sqrt{1,4,16, \ldots} \times 3 \times \sigma_{r m s}$. Blue contours are the radio emission from SUMSS at $843 \mathrm{MHz}$ starting at $0.004 \mathrm{mJy}_{\text {beam }}{ }^{-1}$ and are spaced by factor of 2 intervals. The SUMSS image of the cluster RXCJ0145.0-5300 was heavily affected by artefacts in the diffuse emission region, thus we did not overlay the SUMSS contours on this image. Green contours represent the X-ray emission of the cluster. The background is the DSS blue image. 


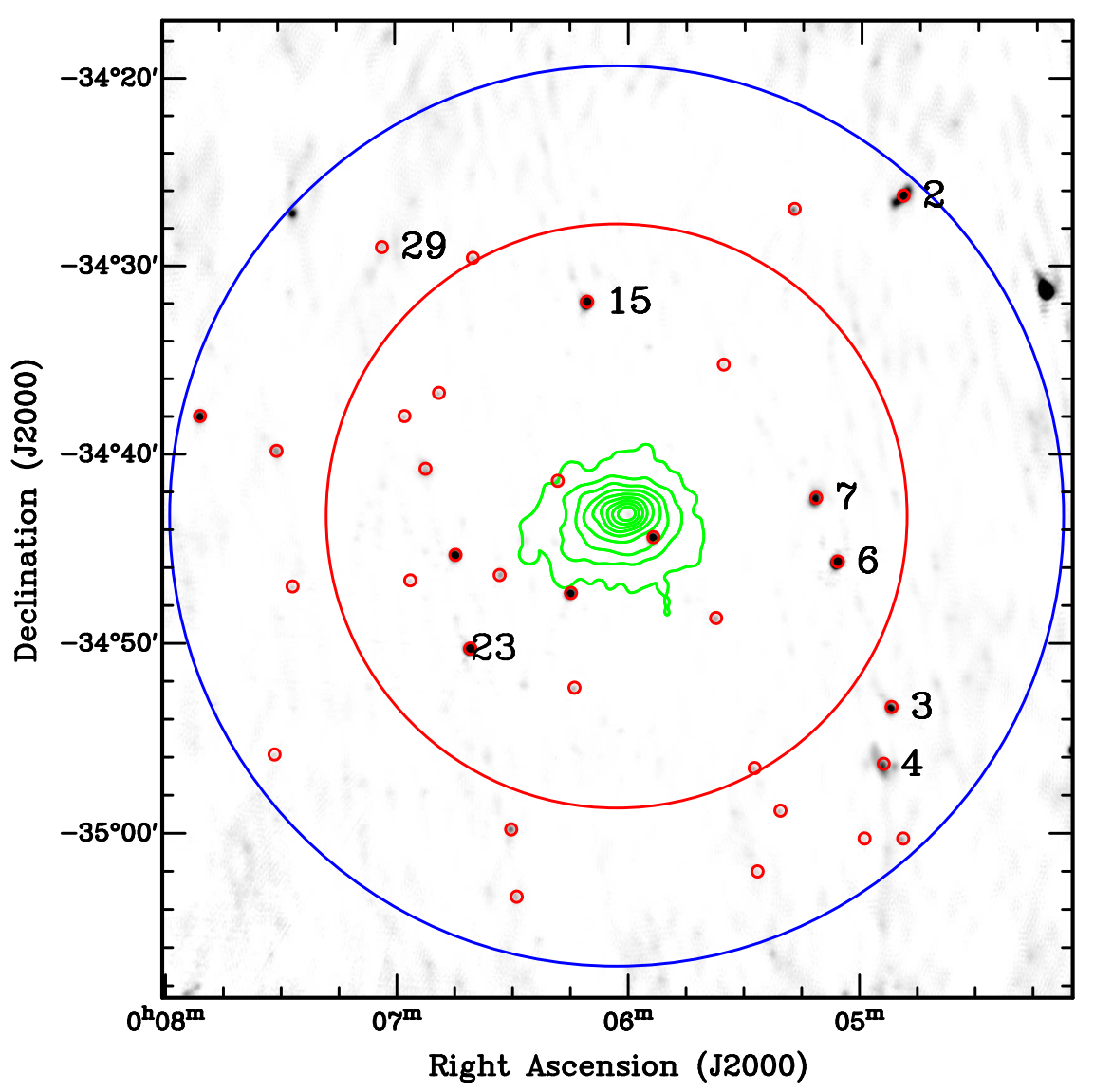

Figure 4.3: RXCJ0006.0-3443: Positions of all detected radio sources and radio IDs of extended sources are annotated on the radio image $(1.4 \mathrm{GHz})$ with small red open circles. The X-ray contours in green are also overlaid on the full resolution radio image. The blue circle represent the ATCA primary beam at $1.4 \mathrm{GHz}$ and the large red circle shows the $2 \mathrm{Mpc}$ radius around the cluster. 

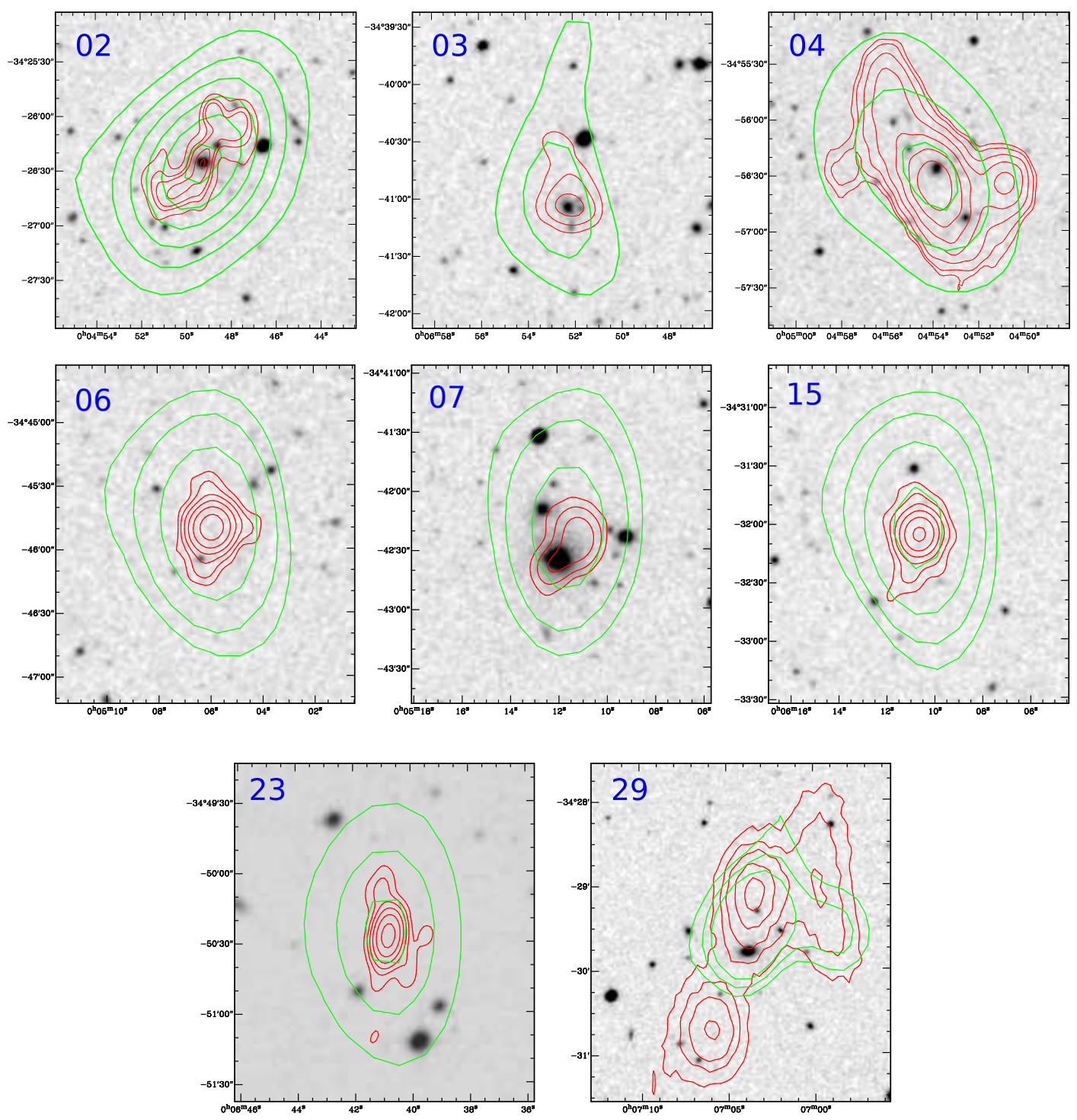

Figure 4.4: Postage stamps of extended radio sources in RXCJ0006.0-3443: red contours demonstrate the radio emission of detected extended sources in the $1.4 \mathrm{GHz}$ ATCA image (beam size of $\left.14^{\prime \prime} \times 8^{\prime \prime}\right)$. Green contours shows the SUMSS radio emission at $843 \mathrm{MHz}$ superimposed on the DSS blue image starting at $4 \mathrm{mJy}^{\text {beam }}{ }^{-1}$ and are spaced in intervals increasing by a factor of 2 . The red contours start at $5 \times \sigma_{r m s}$ and are spaced in intervals increasing by factor of 2 . Bottom right plot is the beam of the high resolution image. 


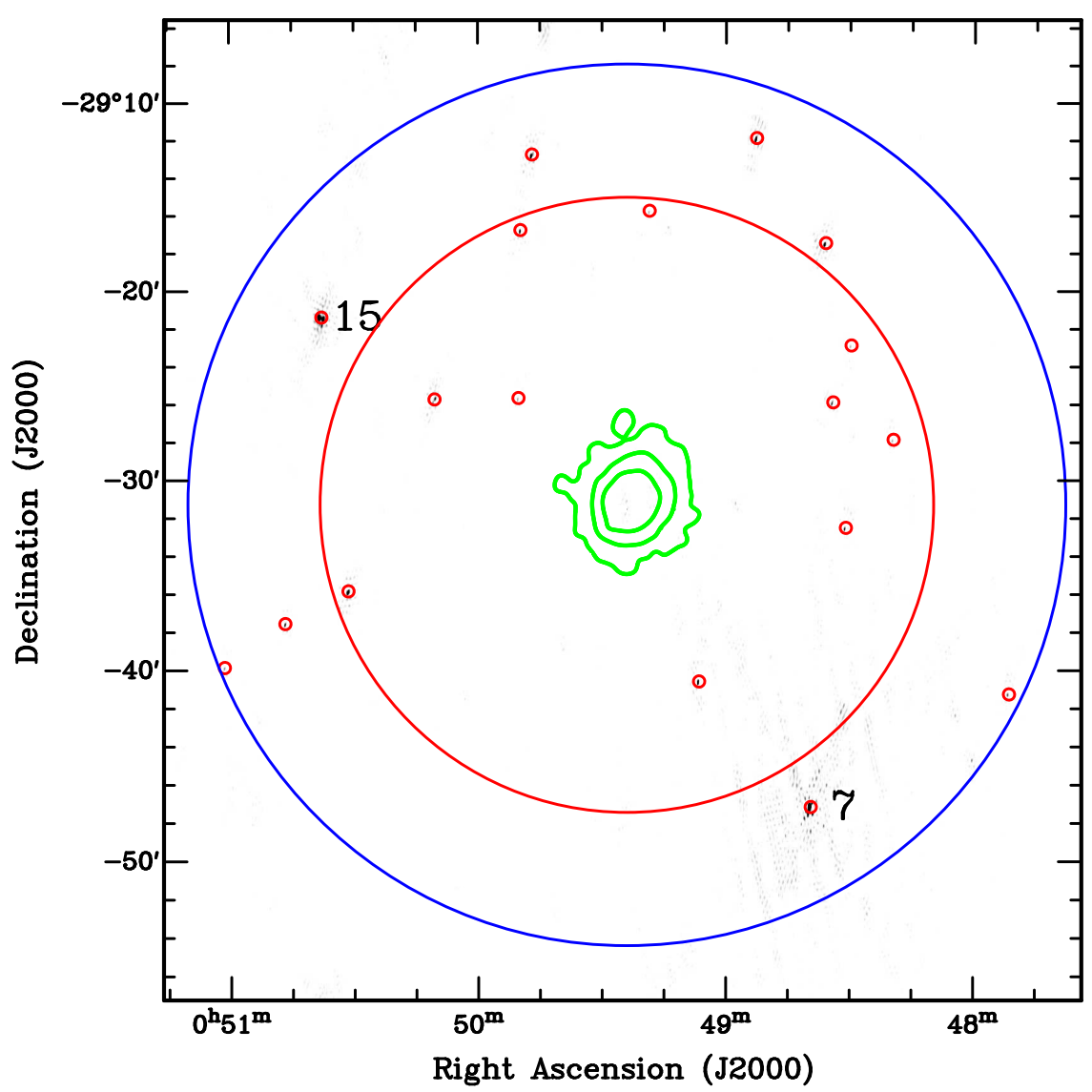

Figure 4.5: RXCJ0049.4-2931: Positions of all detected radio sources and radio IDs of extended sources are annotated on the radio image $(1.4 \mathrm{GHz})$ with small red open circles. The X-ray contours in green are also overlaid on the full resolution radio image. The blue circle represent the ATCA primary beam at $1.4 \mathrm{GHz}$ and the large red circle shows the $2 \mathrm{Mpc}$ radius around the cluster.
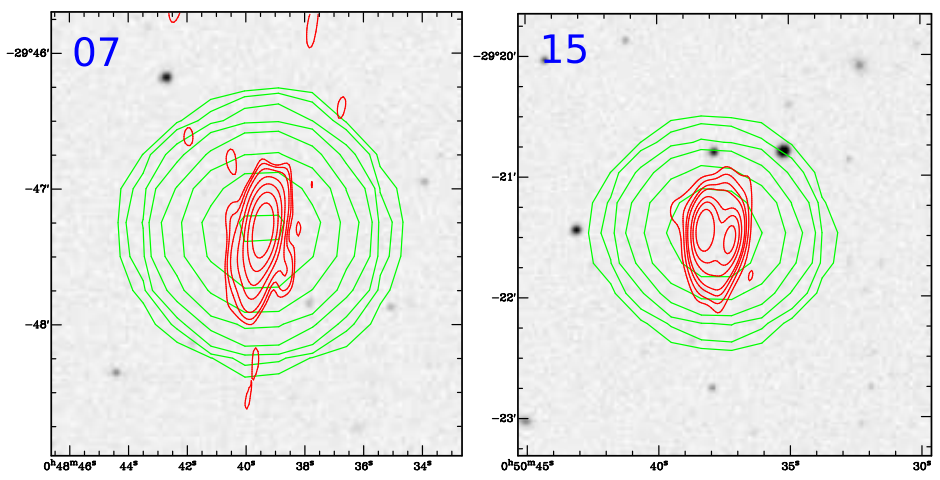

Figure 4.6: Postage stamps of extended radio sources in RXCJ0049.4-2931: red contours demonstrate the radio emission of detected extended sources in the $1.4 \mathrm{GHz}$ ATCA image (beam size of $\left.11^{\prime \prime} \times 4^{\prime \prime}\right)$. Green contours show the NVSS radio emission at $1.4 \mathrm{GHz}$ superimposed on the DSS blue image starting at $4 \mathrm{mJy}_{\text {beam }}{ }^{-1}$ and are spaced in intervals increasing by a factor of 2. The red contours start at $4 \times \sigma_{r m s}$ and are spaced in intervals increasing by factor of 2 . 


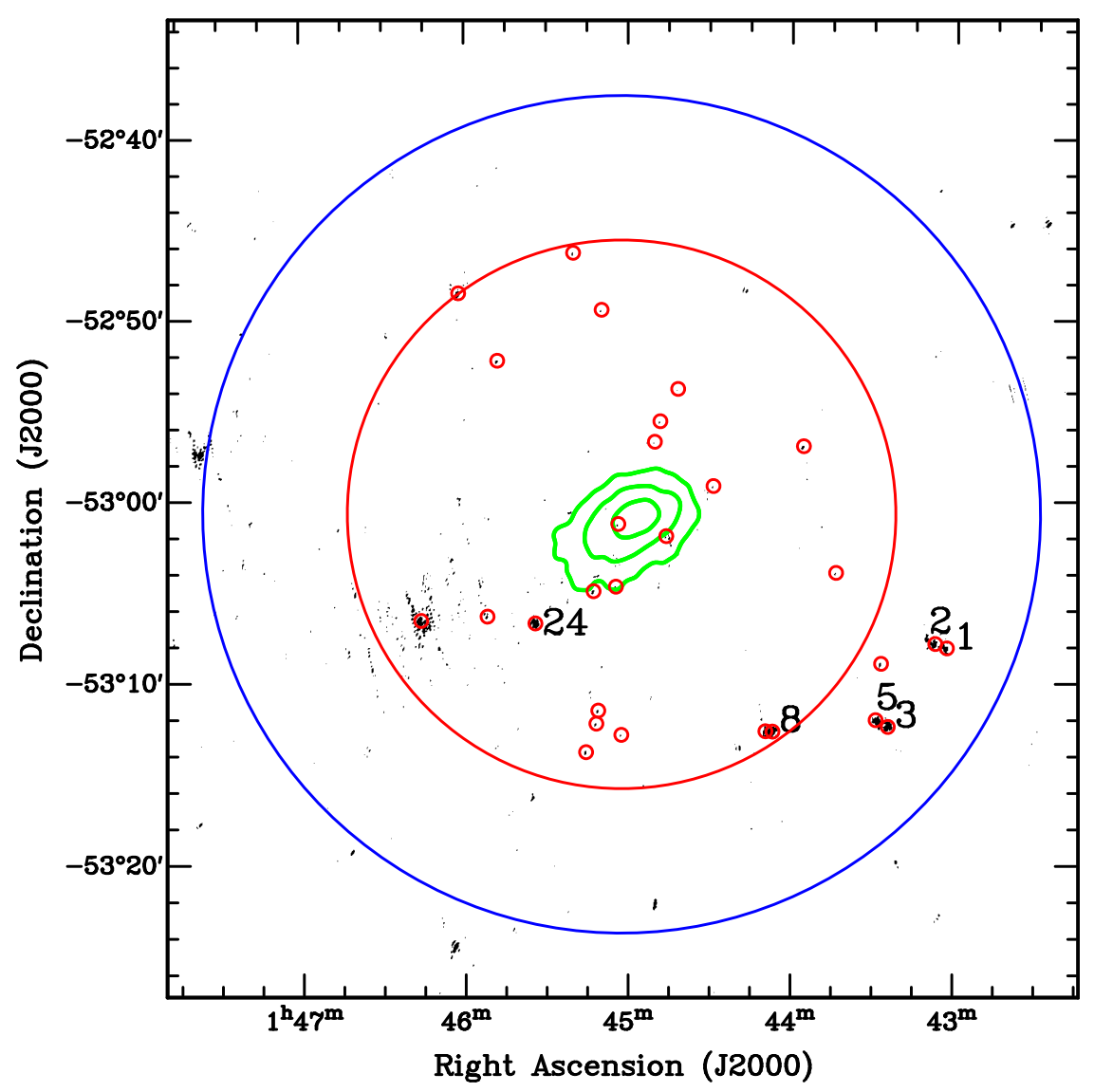

Figure 4.7: RXCJ0145.0-5300: Positions of all detected radio sources and radio IDs of extended sources are annotated on the radio image $(1.4 \mathrm{GHz})$ with small red open circles. The X-ray contours in green are also overlaid on the full resolution radio image. The blue circle represent the ATCA primary beam at $1.4 \mathrm{GHz}$ and the large red circle shows the $2 \mathrm{Mpc}$ radius around the cluster. 

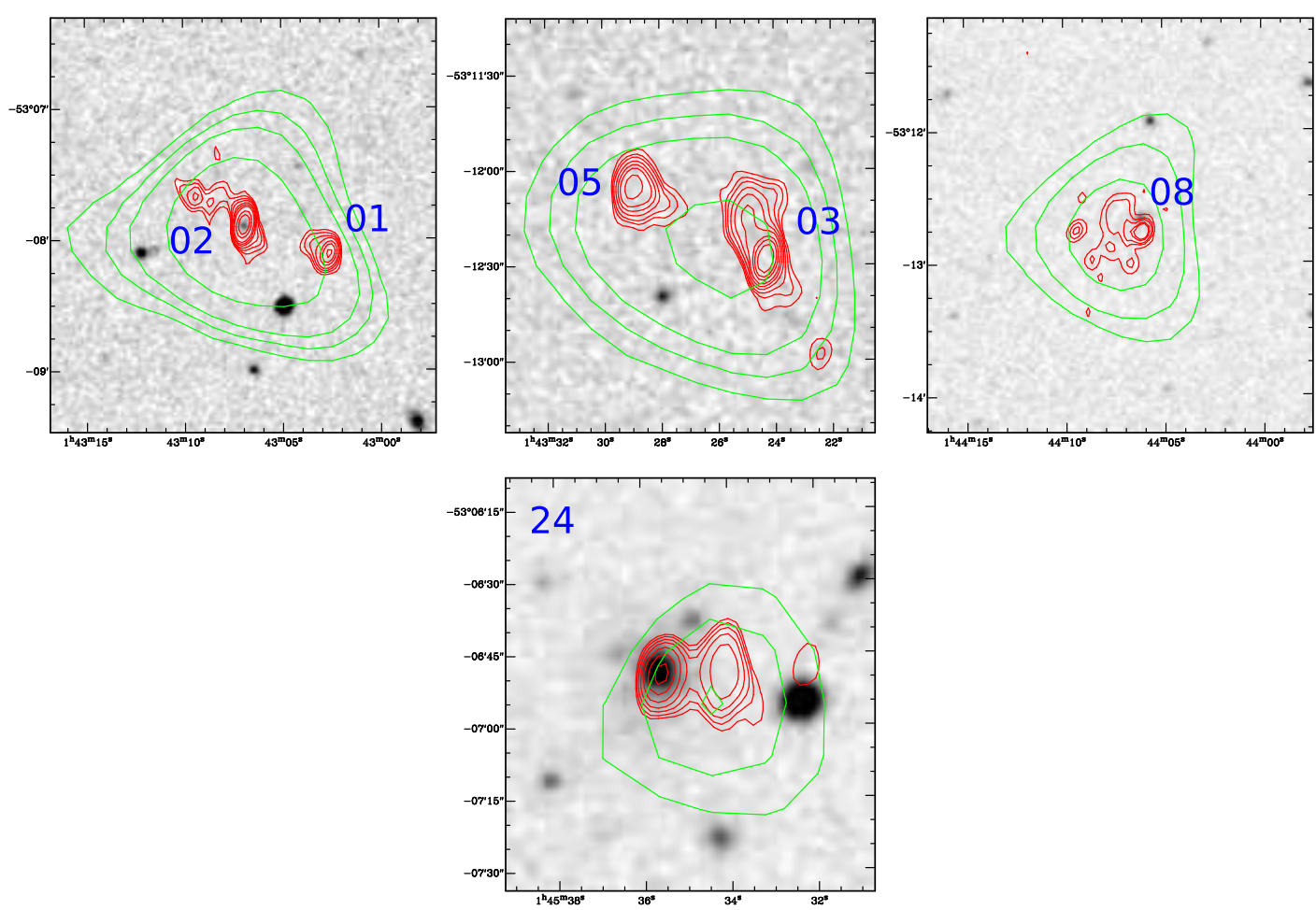

Figure 4.8: Postage stamps of extended radio sources in RXCJ0145.0-5300: red contours demonstrate the radio emission of detected extended sources in the $1.4 \mathrm{GHz}$ ATCA image (beam size of $\left.6^{\prime \prime} \times 4^{\prime \prime}\right)$. Green contours shows the SUMSS radio emission at $843 \mathrm{MHz}$. The background is the DSS blue image starting at $4 \mathrm{mJy}^{\text {beam }}{ }^{-1}$ and are spaced in intervals increasing by a factor of 2 . The red contours start at $5 \times \sigma_{r m s}$ and are spaced in intervals increasing by factor of 2 . 


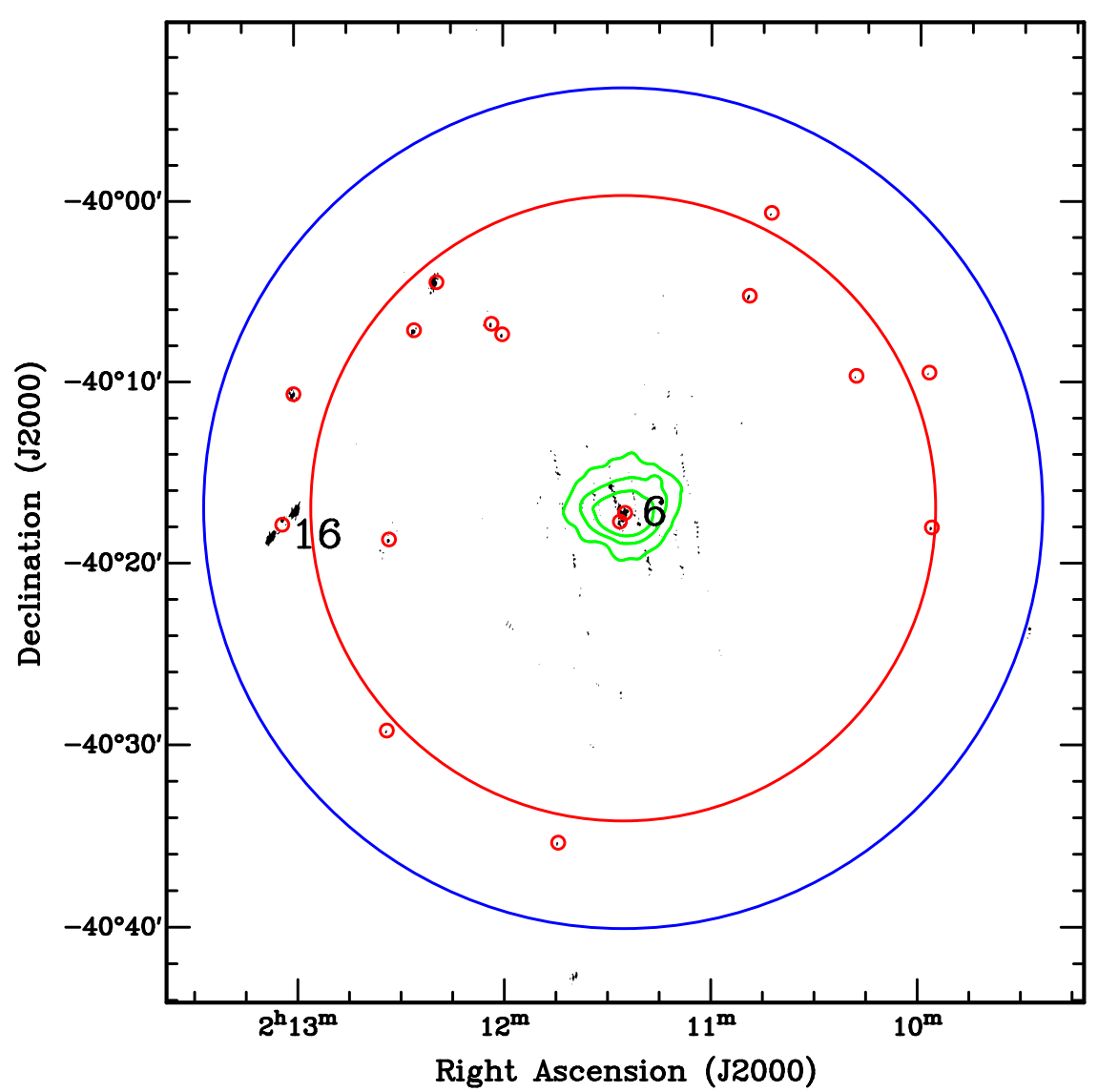

Figure 4.9: RXCJ0211.4-4017: Positions of all detected radio sources and radio IDs of extended sources are annotated on the radio image $(1.4 \mathrm{GHz})$ with small red open circles. The X-ray contours in green are also overlaid on the full resolution radio image. The blue circle represent the ATCA primary beam at $1.4 \mathrm{GHz}$ and the large red circle shows the $2 \mathrm{Mpc}$ radius around the cluster.
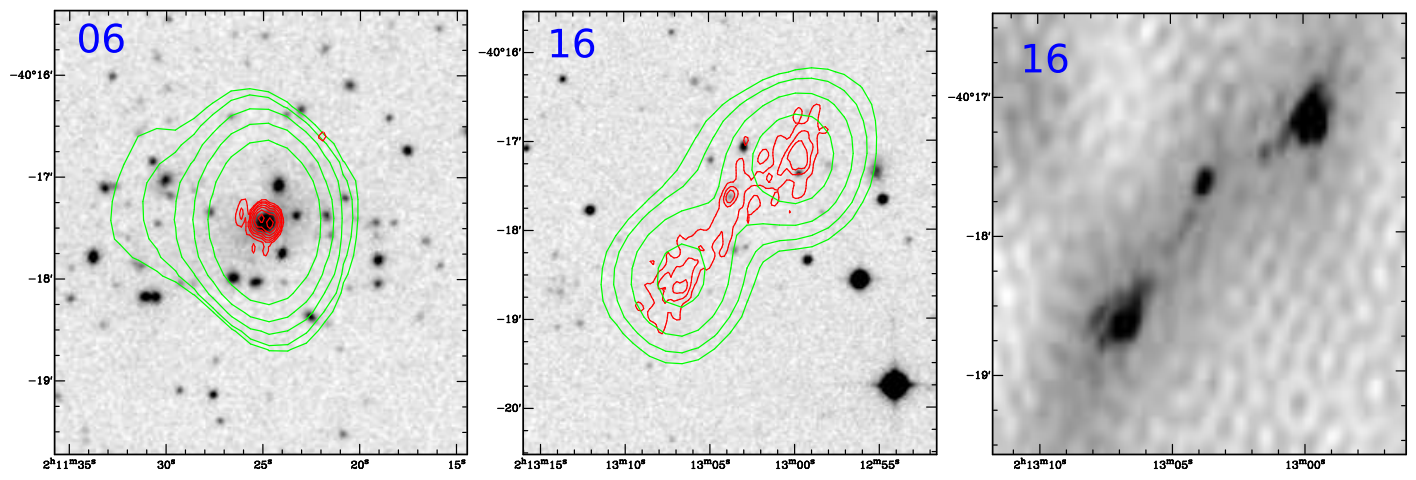

Figure 4.10: Postage stamps of extended radio sources in RXCJ0211.4-4017: red contours demonstrate the radio emission of detected extended sources in the $1.4 \mathrm{GHz}$ ATCA image (beam size of $8^{\prime \prime} \times 4^{\prime \prime}$ ). Green contours shows the SUMSS radio emission at $843 \mathrm{MHz}$. The background is the DSS blue image starting at $4 \mathrm{mJy}^{\text {beam }}{ }^{-1}$ and are spaced in intervals increasing by a factor of 2 . The red contours start at $4 \times \sigma_{r m s}$ and are spaced in intervals increasing by factor of 2 . Right plot shows the actual radio emission of the Source 16. 


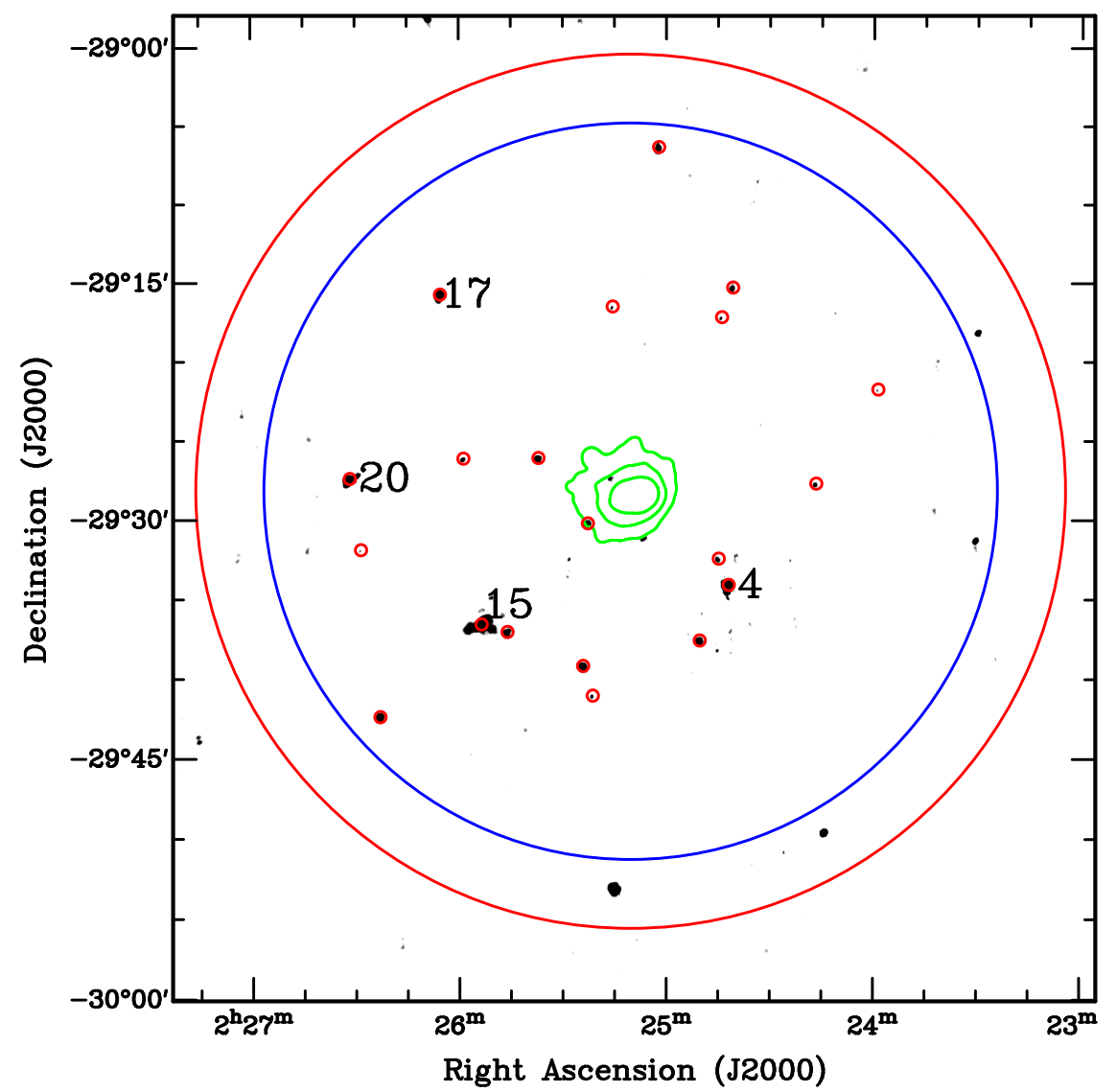

Figure 4.11: RXCJ0225.1-2928: Positions of all detected radio sources and radio IDs of extended sources are annotated on the radio image $(1.4 \mathrm{GHz})$ with small red open circles. The X-ray contours in green are also overlaid on the full resolution radio image. The blue circle represent the ATCA primary beam at $1.4 \mathrm{GHz}$ and the large red circle shows the $2 \mathrm{Mpc}$ radius around the cluster. 

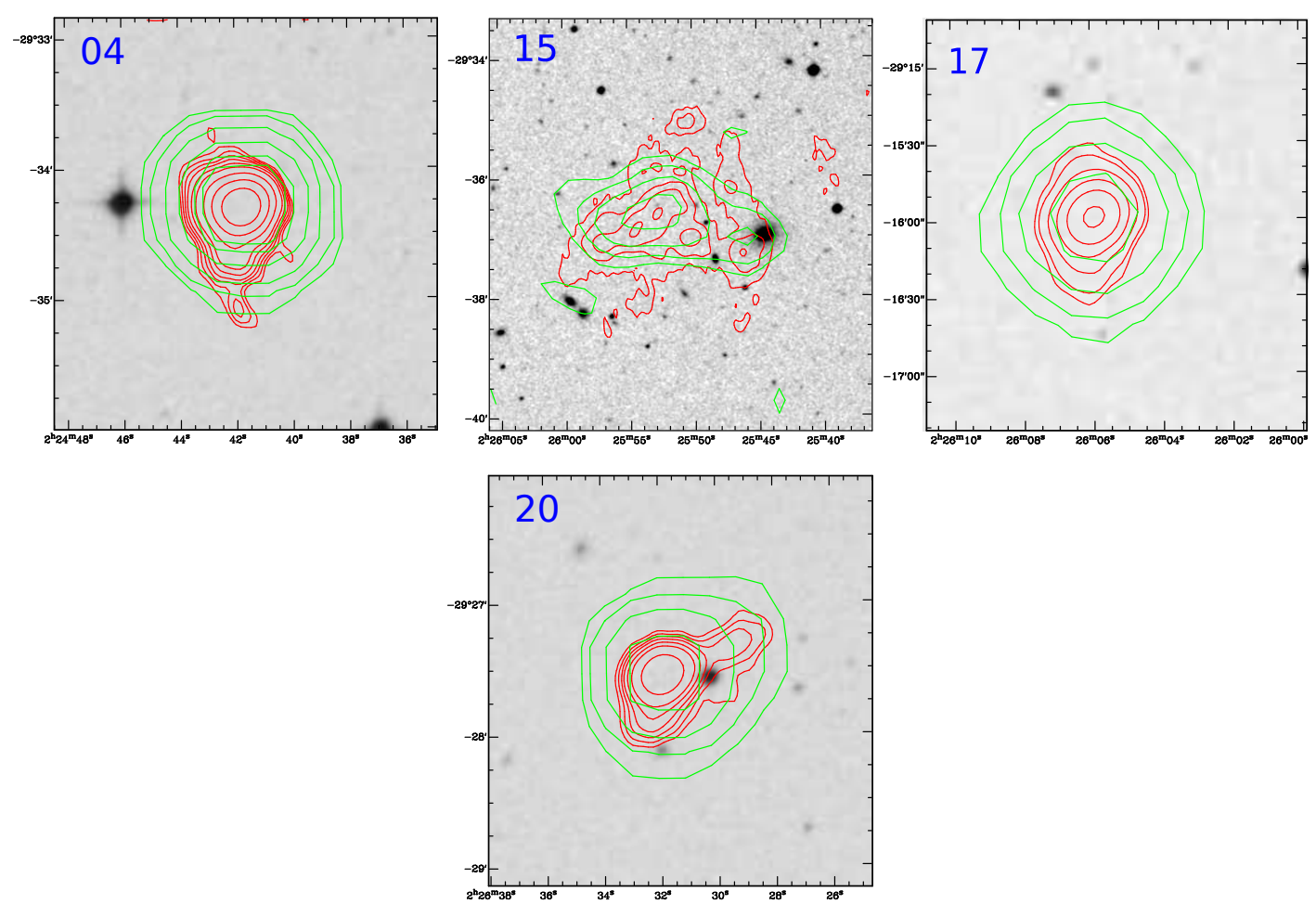

Figure 4.12: Postage stamps of extended radio sources in RXCJ0225.1-2928: red contours demonstrate the radio emission of detected extended sources in the ATCA primary beam with $10^{\prime \prime} \times 4^{\prime \prime}$ at $1.4 \mathrm{GHz}$. The green contours show the NVSS radio emission at $1.4 \mathrm{GHz}$. The background is the DSS blue image starting at $4 \mathrm{mJy}^{\text {beam }}{ }^{-1}$ and are spaced in intervals increasing by a factor of 2 . The red contours start at $4 \times \sigma_{r m s}$ and are spaced in intervals increasing by factor of 2 . 


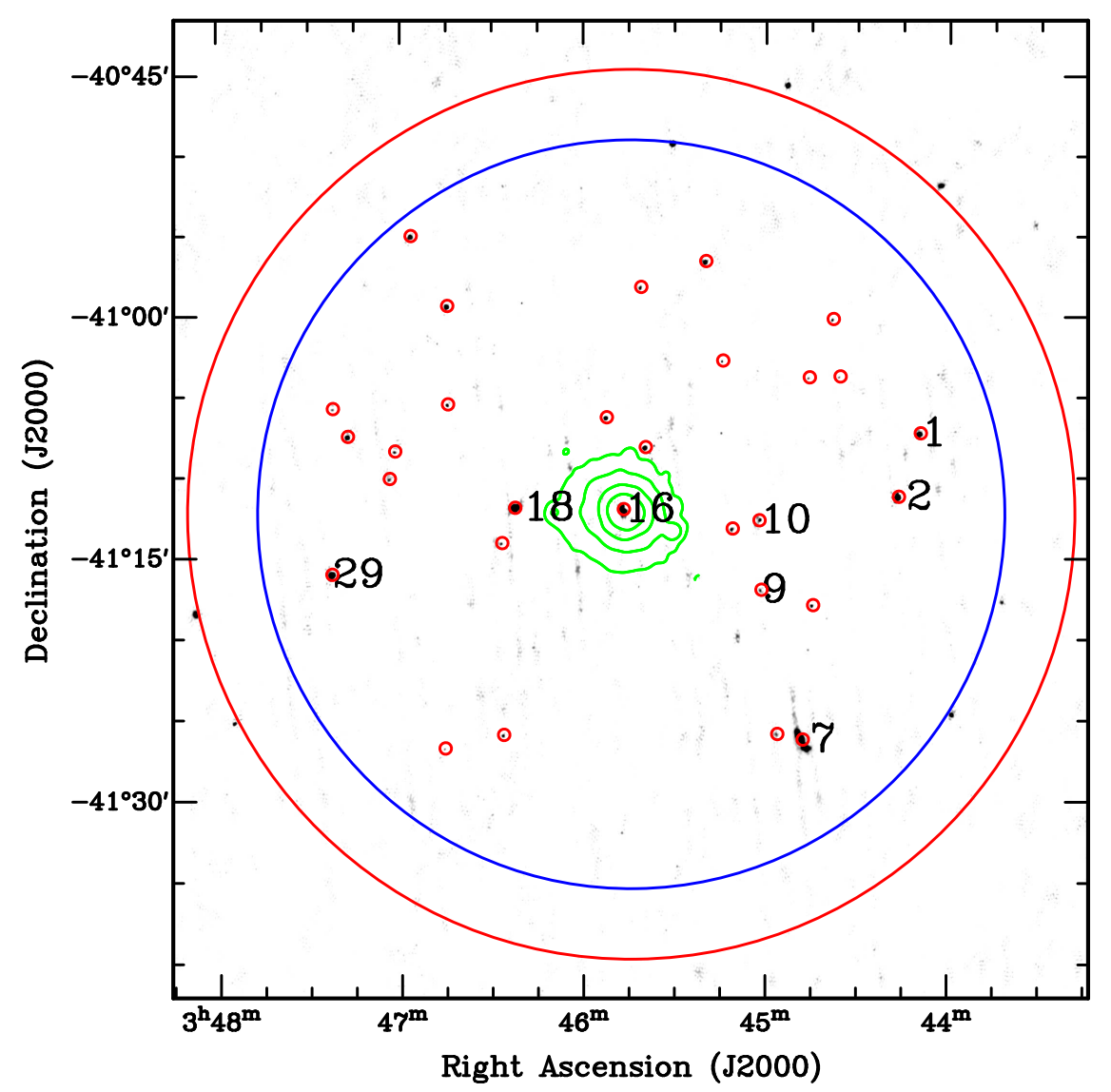

Figure 4.13: RXCJ0345.7-4112: Positions of all detected radio sources and radio IDs of extended sources are annotated on the radio image $(1.4 \mathrm{GHz})$ with small red open circles. The X-ray contours in green are also overlaid on the full resolution radio image. The blue circle represent the ATCA primary beam at $1.4 \mathrm{GHz}$ and the large red circle shows the $2 \mathrm{Mpc}$ radius around the cluster. 

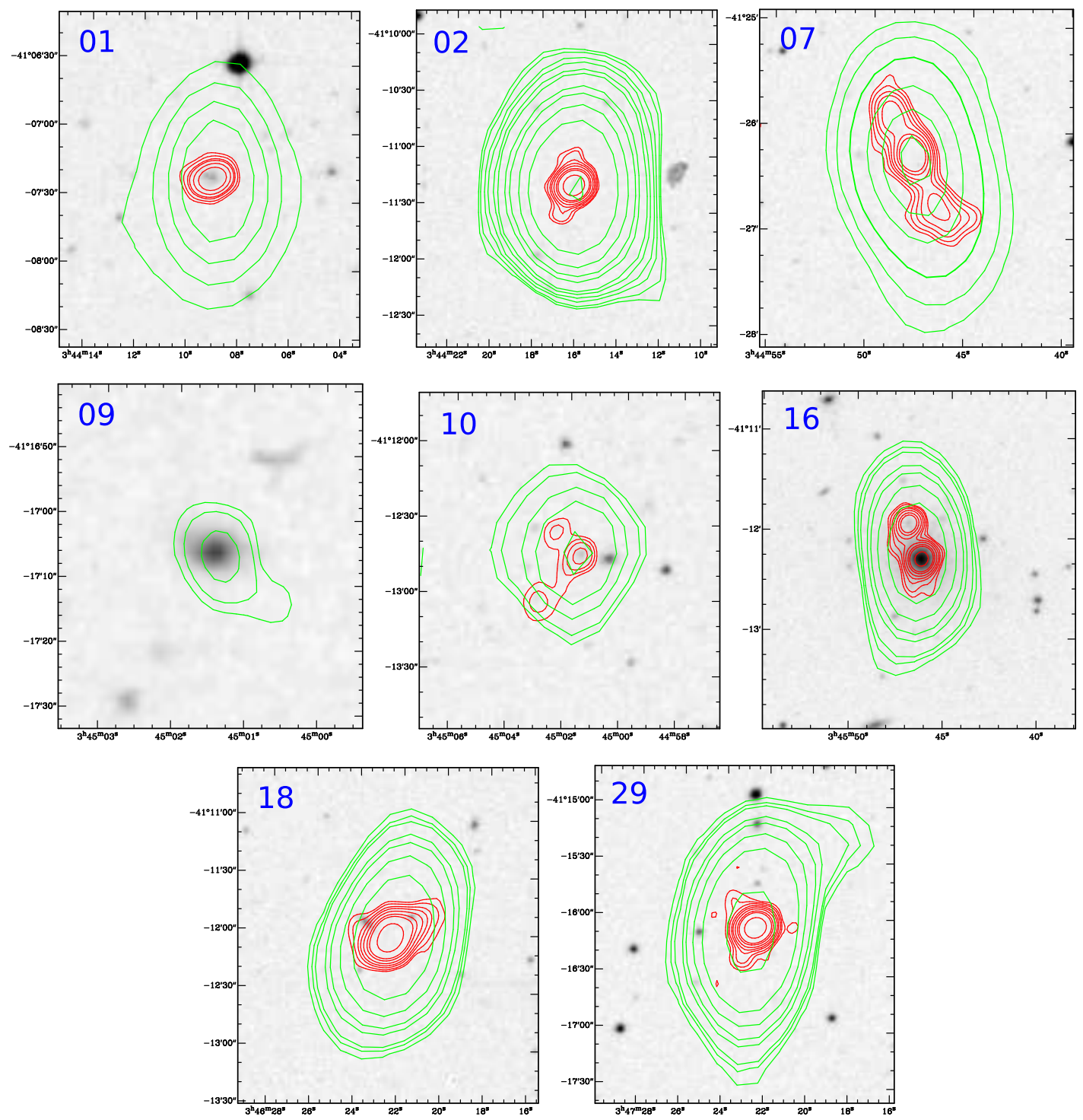

Figure 4.14: Postage stamps of extended radio sources in RXCJ0345.7-4112: red contours demonstrate the radio emission of detected extended sources in the ATCA primary beam with $10^{\prime \prime} \times 11^{\prime \prime}$ at $1.4 \mathrm{GHz}$. Green contours show the SUMSS radio emission at $843 \mathrm{MHz}$. The background is the DSS blue image starting at $4 \mathrm{mJy}_{\text {beam }}{ }^{-1}$ and are spaced in intervals increasing by a factor of 2. The red contours start at $4 \times \sigma_{r m s}$ and are spaced in intervals increasing by factor of 2 . 


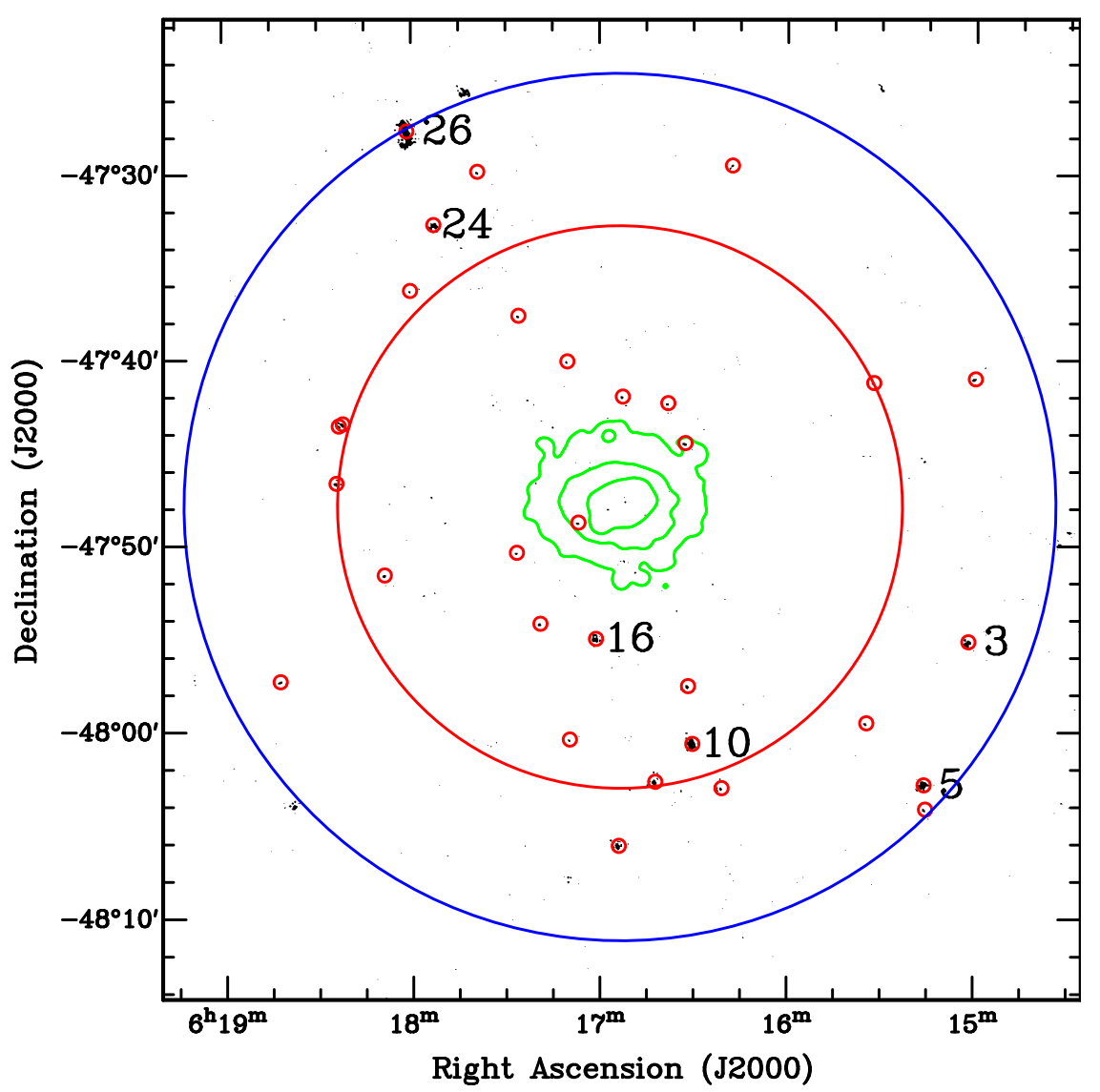

Figure 4.15: RXCJ0616.8-4748: Positions of all detected radio sources and radio IDs of extended sources are annotated on the radio image $(1.4 \mathrm{GHz})$ with small red open circles. The X-ray contours in green are also overlaid on the full resolution radio image. The blue circle represent the ATCA primary beam at $1.4 \mathrm{GHz}$ and the large red circle shows the $2 \mathrm{Mpc}$ radius around the cluster. 

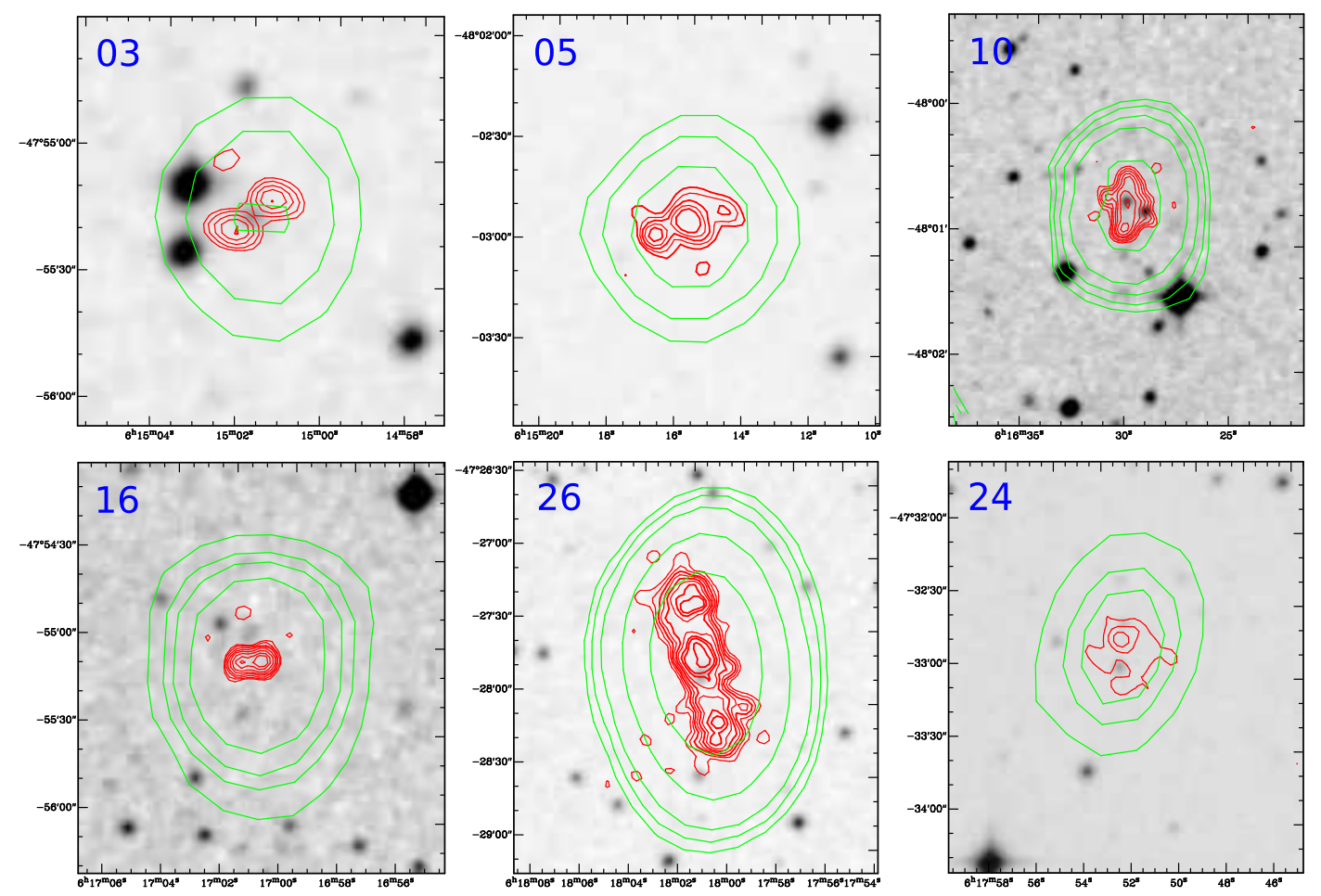

Figure 4.16: Postage stamps of extended radio sources in RXCJ0616.8-4748: red contours demonstrate the radio emission of detected extended sources in the $1.4 \mathrm{GHz}$ ATCA image (beam size of $\left.6^{\prime \prime} \times 5^{\prime \prime}\right)$. Green contours show the SUMSS radio emission at $843 \mathrm{MHz}$. The background is the DSS blue image starting at $4 \mathrm{mJy} \mathrm{beam}^{-1}$ and are spaced in intervals increasing by a factor of 2 . The red contours start at $4 \times \sigma_{r m s}$ and are spaced in intervals increasing by factor of 2 . 


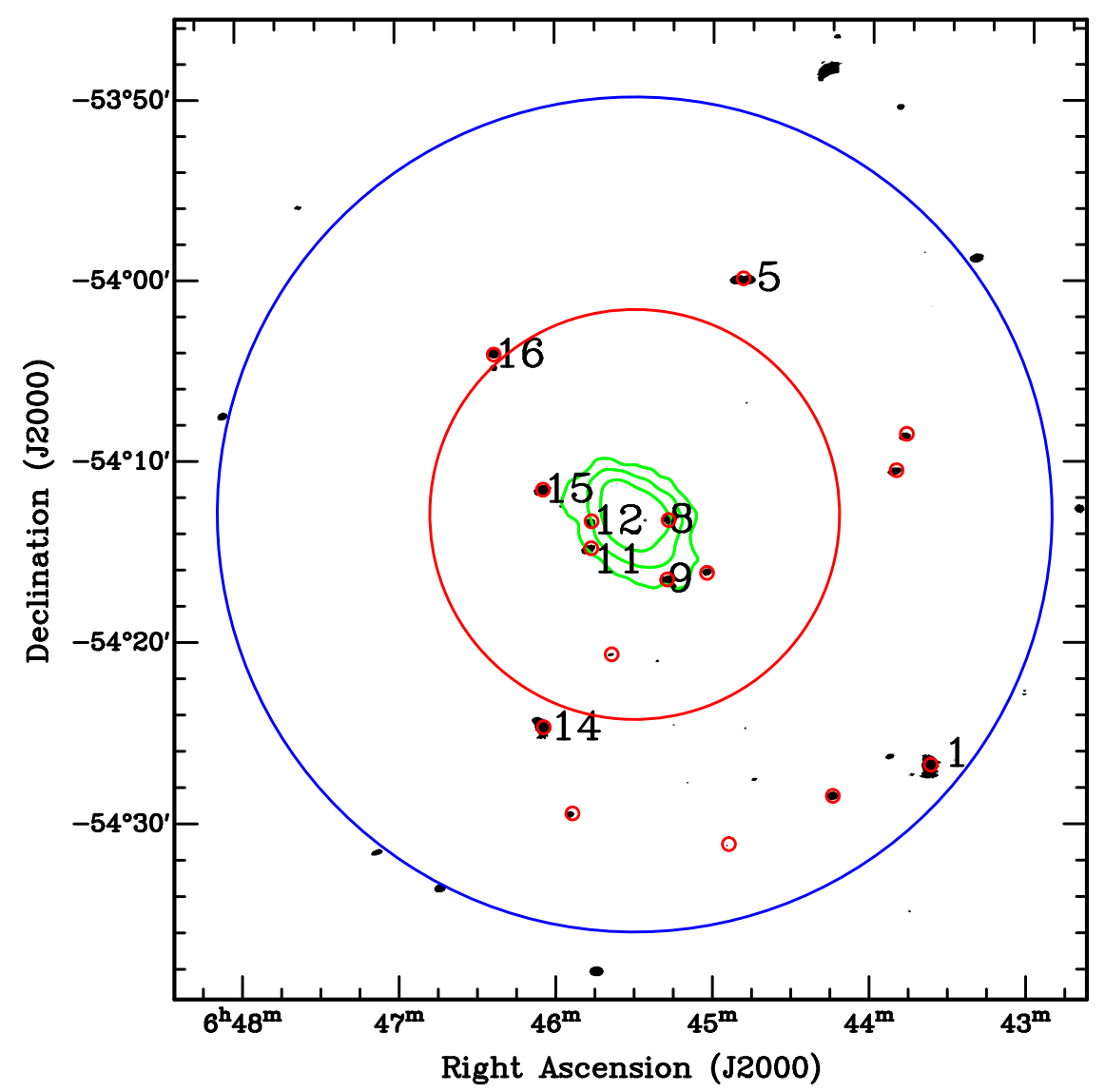

Figure 4.17: RXCJ0645.4-5413: Positions of all detected radio sources and radio IDs of extended sources are annotated on the radio image $(1.4 \mathrm{GHz})$ with small red open circles. The X-ray contours in green are also overlaid on the full resolution radio image. The blue circle represent the ATCA primary beam at $1.4 \mathrm{GHz}$ and the large red circle shows the $2 \mathrm{Mpc}$ radius around the cluster. 

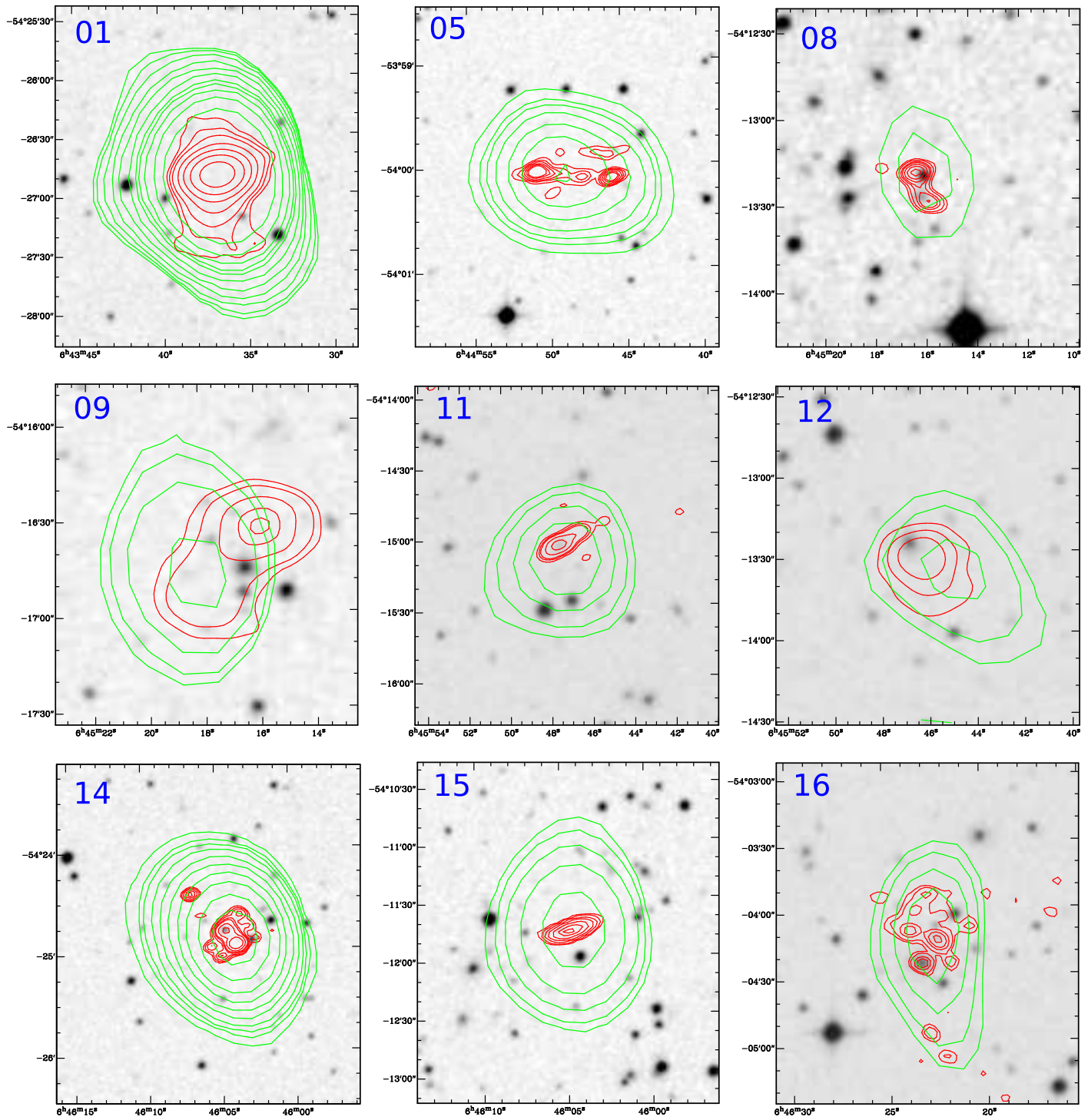

Figure 4.18: Postage stamps of extended radio sources in RXCJ0645.4-5413: red contours demonstrate the radio emission of detected extended sources in the $1.4 \mathrm{GHz}$ ATCA image (beam size of $\left.7^{\prime \prime} \times 4^{\prime \prime}\right)$. Green contours show the SUMSS radio emission at $843 \mathrm{MHz}$. The background is the DSS blue image starting at $4 \mathrm{mJy}^{\text {beam }}{ }^{-1}$ and are spaced in intervals increasing by a factor of 2 . Red contours start at $5 \times \sigma_{r m s}$ and are spaced in intervals increasing by factor of 2 . 


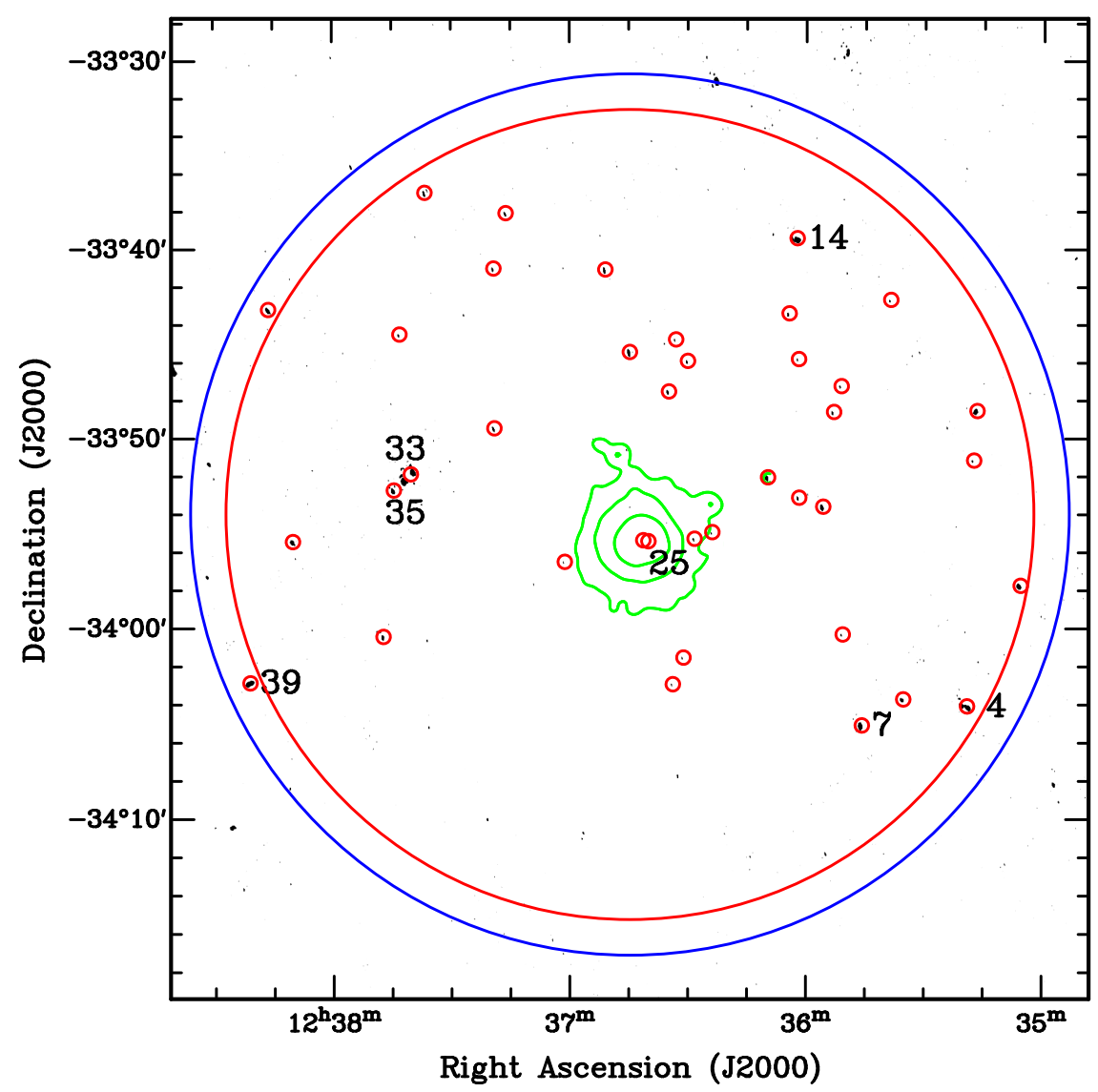

Figure 4.19: RXCJ1236.7-3354: Positions of the all detected radio sources and the radio IDs of extended sources are annotated on the radio image $(1.4 \mathrm{GHz})$ with red open circles. The X-ray contours in green are also overlaid on the full resolution radio image. The blue circle represent the ATCA primary beam at $1.4 \mathrm{GHz}$ and the large red circle shows the $2 \mathrm{Mpc}$ radius around the cluster. 

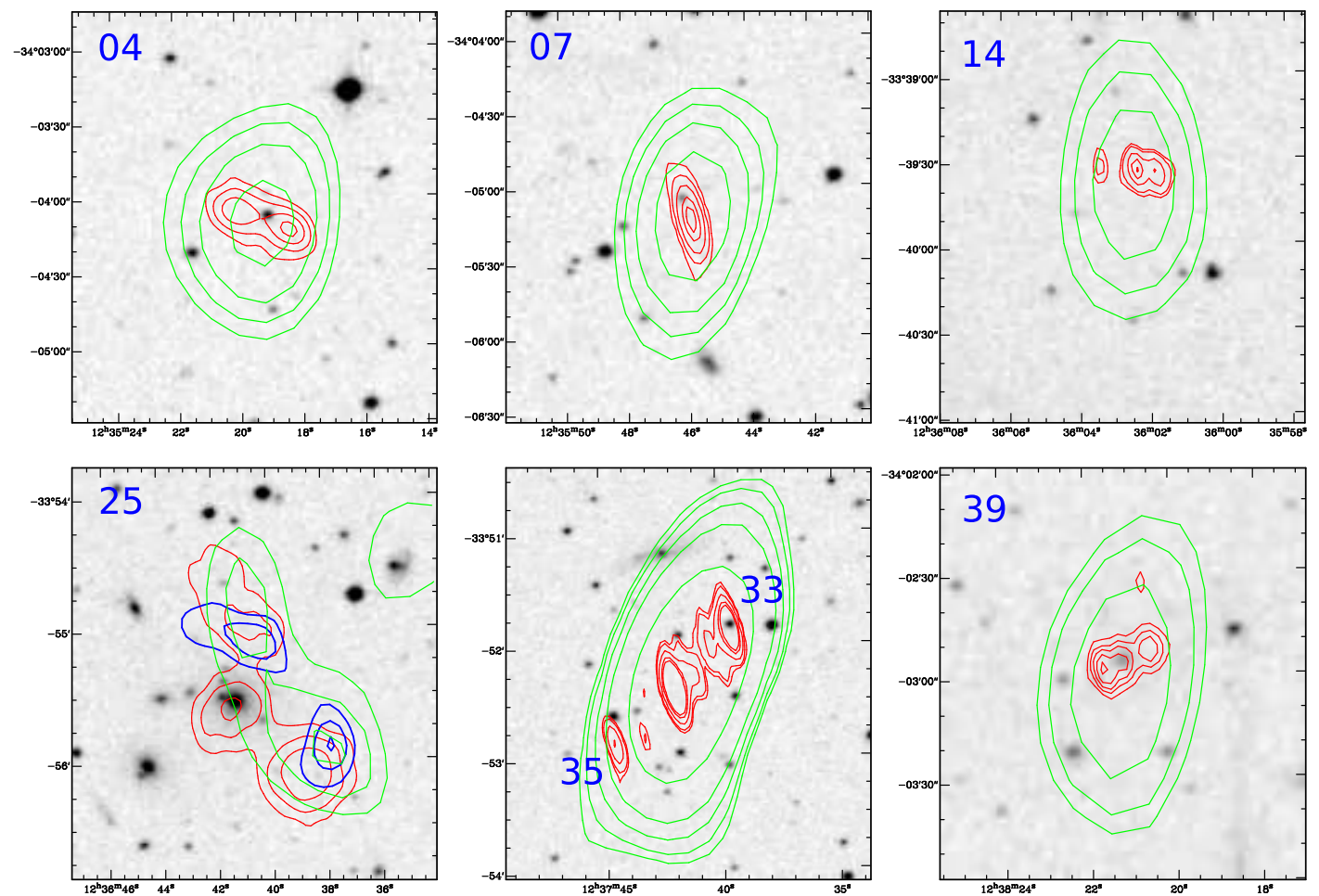

Figure 4.20: Postage stamps of extended radio sources in RXCJ1236.7-3354: red contours demonstrate the radio emission of detected extended sources in the $1.4 \mathrm{GHz}$ ATCA image (beam size of $10^{\prime \prime} \times 4^{\prime \prime}$ ). Green contours show the SUMSS radio emission at $843 \mathrm{MHz}$. The background image is the DSS blue image starting at $4 \mathrm{mJy} \mathrm{beam}^{-1}$ and are spaced in intervals increasing by a factor of 2. The blue contours represents the radio emission at $150 \mathrm{MHz}$ in the TGSS image. The red contours start at $5 \times \sigma_{r m s}$ and are spaced in intervals increasing by factor of 2 . 


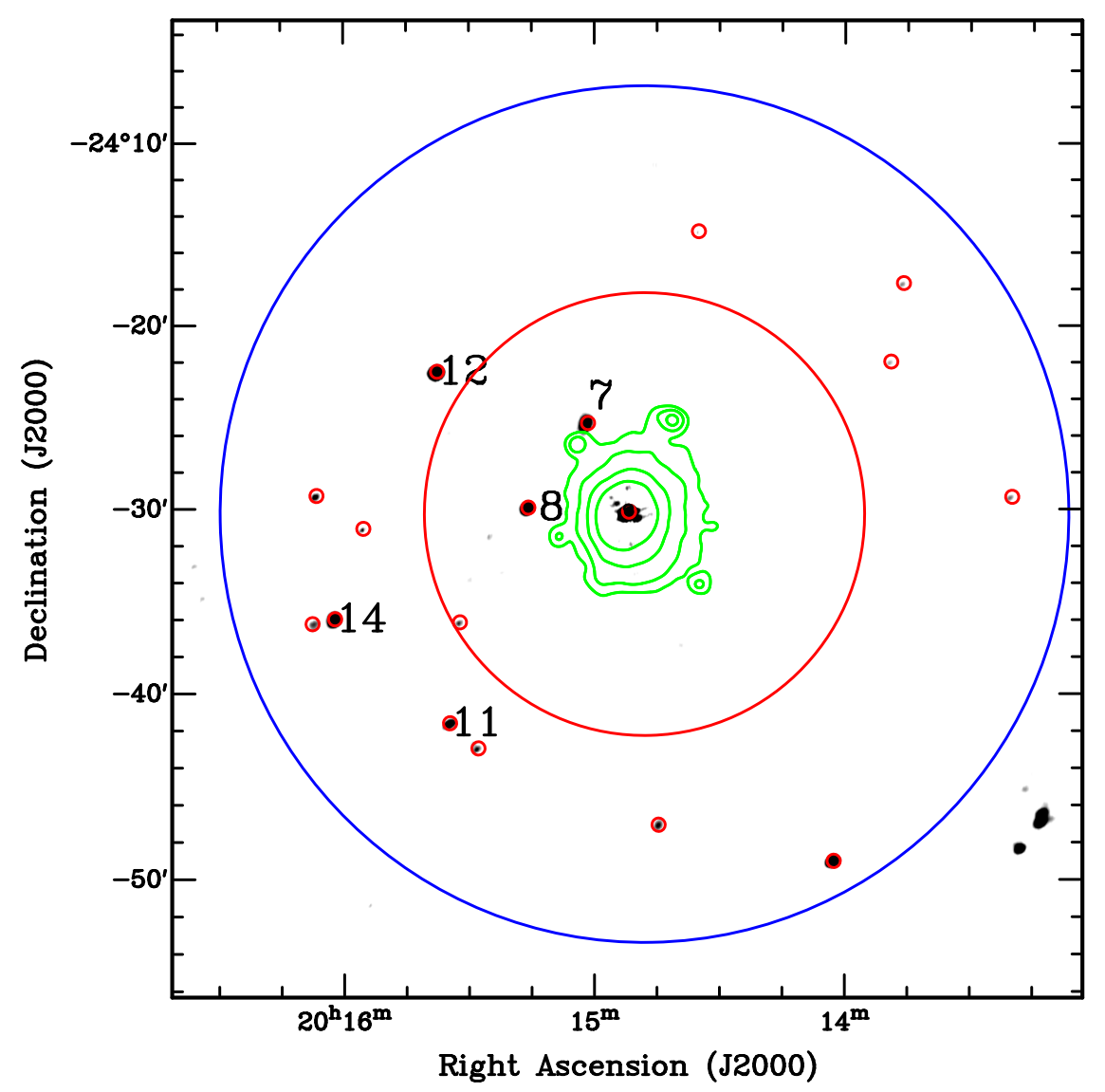

Figure 4.21: RXCJ2014.8-2430: Positions of the all detected radio sources and the radio IDs of extended sources are annotated on the radio image $(1.4 \mathrm{GHz})$ with small red open circles. The $\mathrm{X}$-ray contours in green are also overlaid on the full resolution radio image. The blue circle represent the ATCA primary beam at $1.4 \mathrm{GHz}$ and the large red circle shows the $2 \mathrm{Mpc}$ radius around the cluster. 

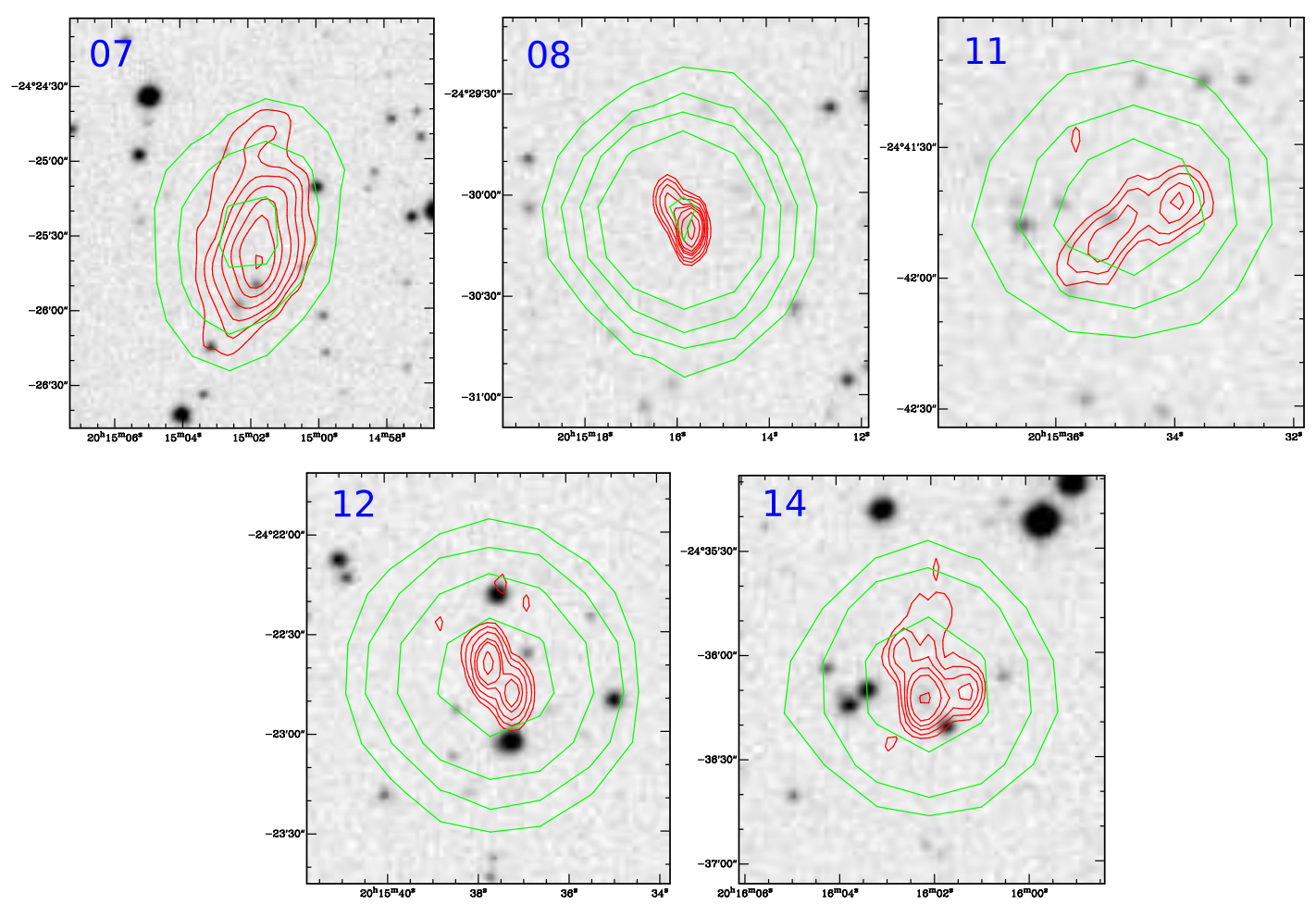

Figure 4.22: Postage stamps of extended radio sources in RXCJ2014.8-2430: red contours demonstrate the radio emission of detected extended sources in the $1.4 \mathrm{GHz}$ ATCA image (beam size of $\left.11^{\prime \prime} \times 4^{\prime \prime}\right)$. Green contours show the NVSS radio emission at $1.4 \mathrm{GHz}$. The background image is the DSS blue image starting at $4 \mathrm{mJy} \mathrm{beam}^{-1}$ and are spaced in intervals increasing by a factor of 2 . The red contours start at $4 \times \sigma_{r m s}$ and are spaced in intervals increasing by factor of 2 . 


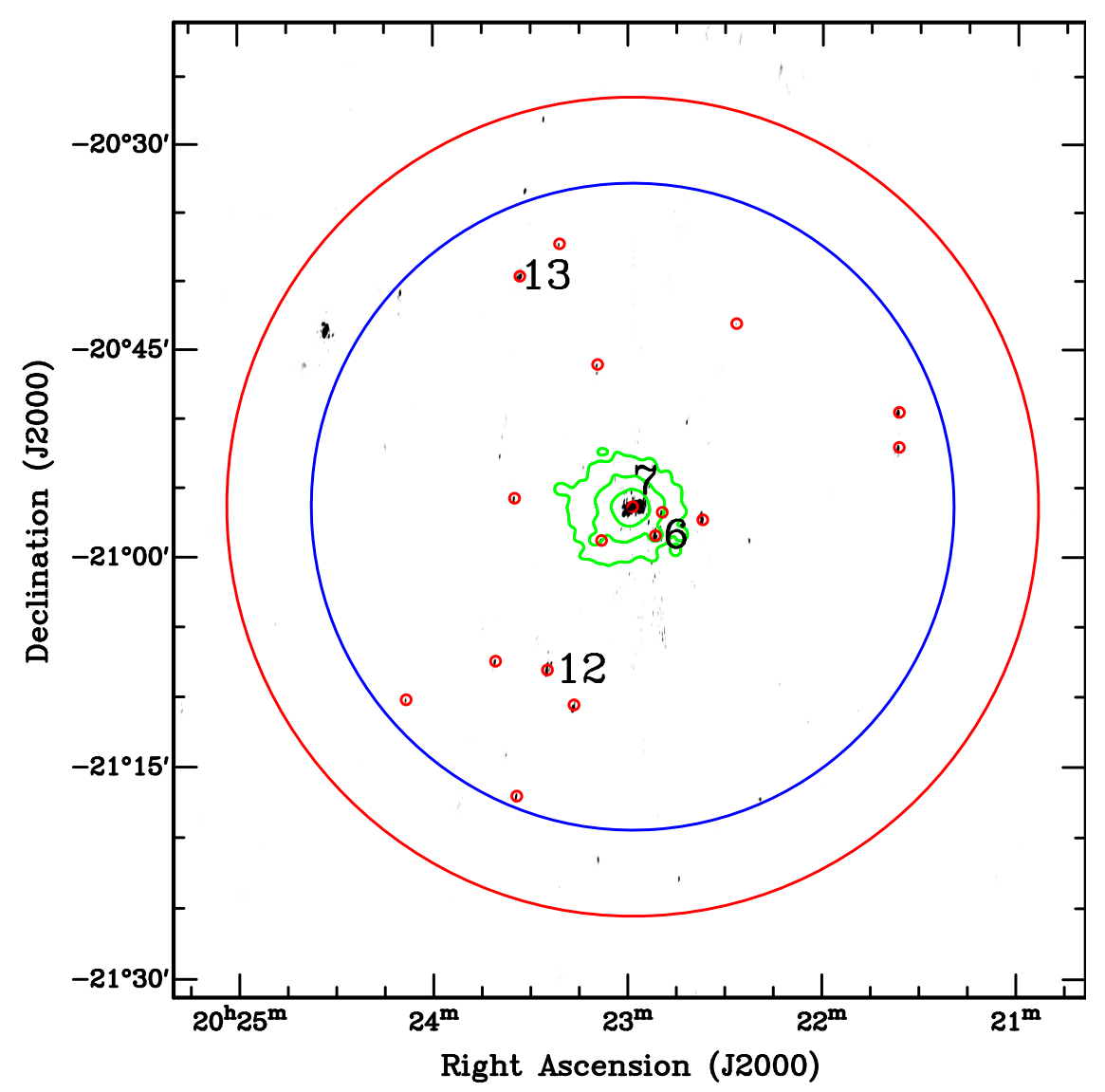

Figure 4.23: RXCJ2023.0-2056: Positions of the all detected radio sources and the radio IDs of extended sources are annotated on the radio image $(1.4 \mathrm{GHz})$ with small red open circles. The $\mathrm{X}$-ray contours in green are also overlaid on the full resolution radio image. The blue circle represent the ATCA primary beam at $1.4 \mathrm{GHz}$ and the large red circle shows the $2 \mathrm{Mpc}$ radius around the cluster. 

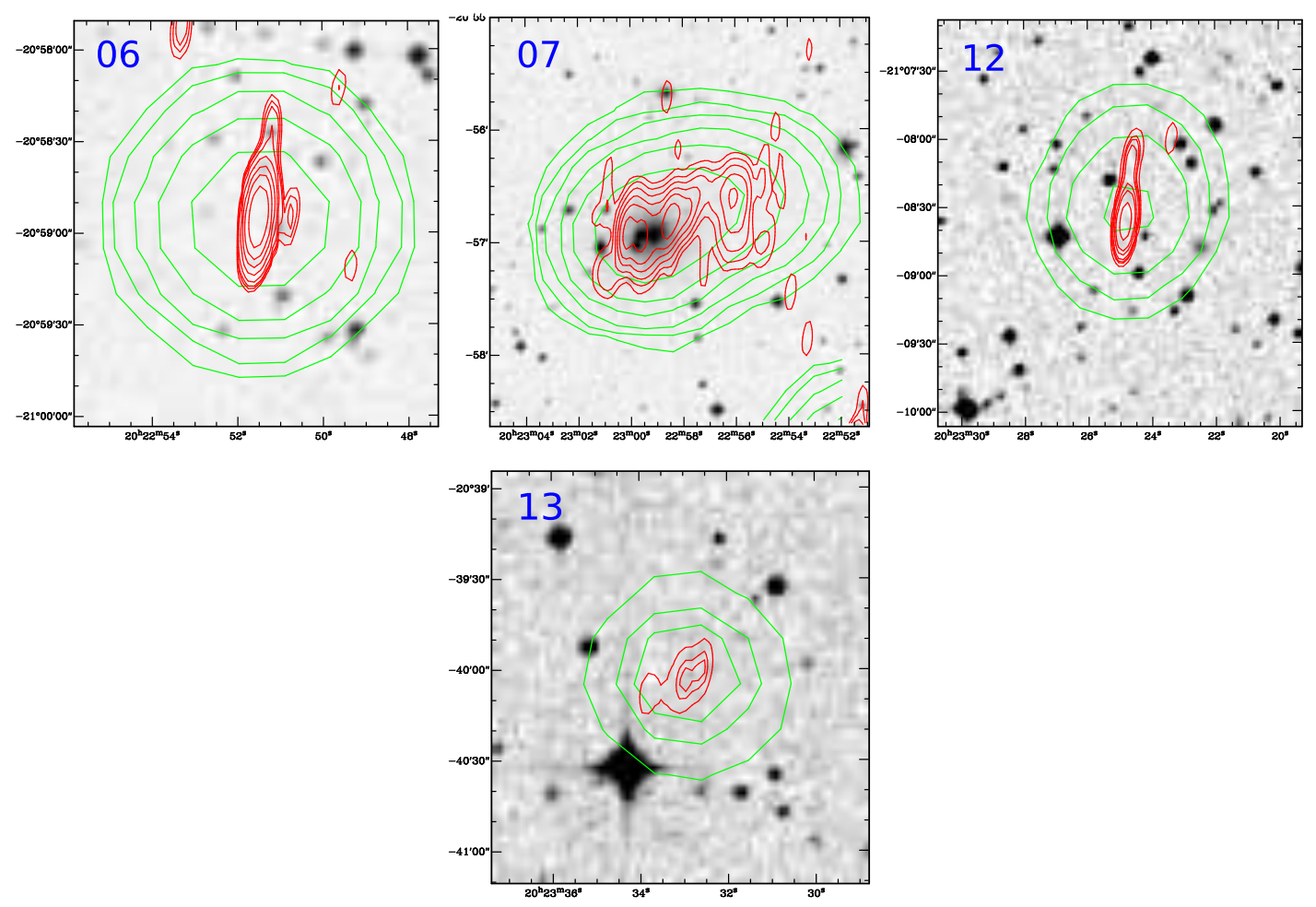

Figure 4.24: Postage stamps of extended radio sources in RXCJ2023.0-2056: red contours demonstrate the radio emission of detected extended sources in the $1.4 \mathrm{GHz}$ ATCA image (beam size of $19^{\prime \prime} \times 5^{\prime \prime}$ ). Green contours show the NVSS radio emission at $1.4 \mathrm{GHz}$. The background image is the DSS blue image starting at $4 \mathrm{mJy} \mathrm{beam}^{-1}$ and are spaced in intervals increasing by a factor of 2 . The red contours start at $5 \times \sigma_{r m s}$ and are spaced in intervals increasing by factor of 2 . 


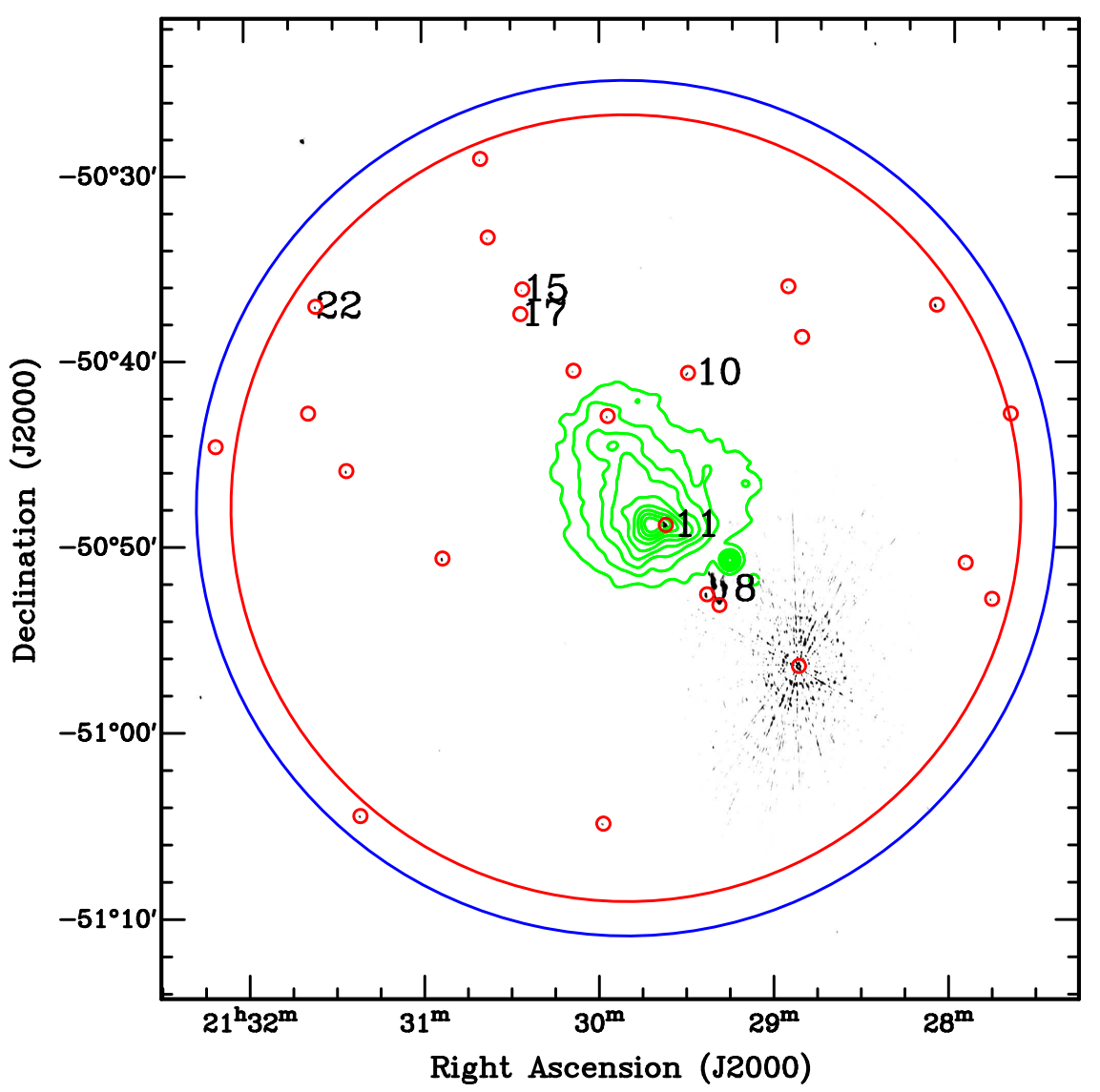

Figure 4.25: RXCJ2129.8-5048: Positions of the all detected radio sources and the radio IDs of extended sources are annotated on the radio image $(1.4 \mathrm{GHz})$ with small red open circles. The $\mathrm{X}$-ray contours in green are also overlaid on the full resolution radio image. The blue circle represent the ATCA primary beam at $1.4 \mathrm{GHz}$ and the large red circle shows the $2 \mathrm{Mpc}$ radius around the cluster. 

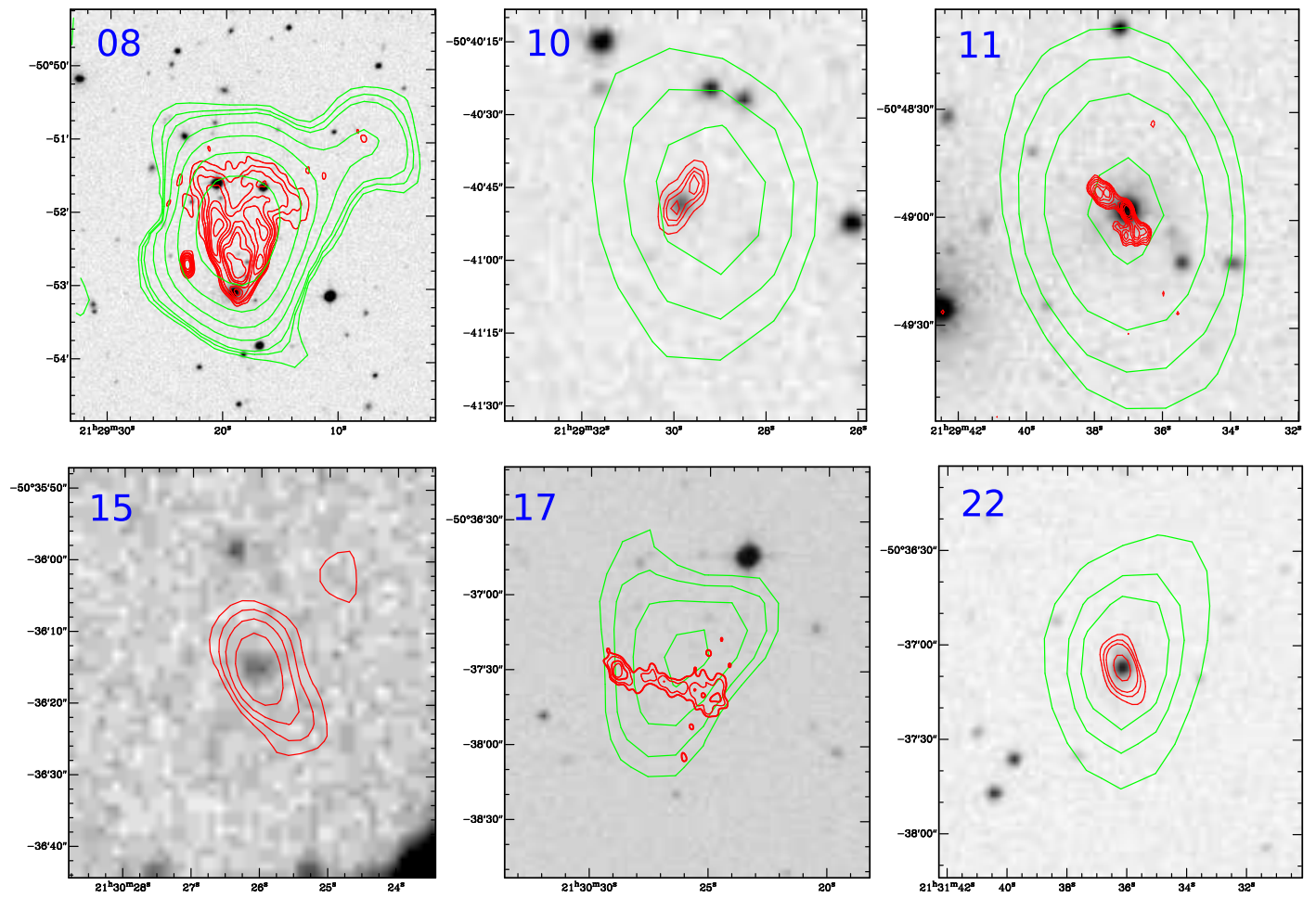

Figure 4.26: Postage stamps of extended radio sources in RXCJ2129.8-5048: red contours demonstrate the radio emission of detected extended sources in the $1.4 \mathrm{GHz}$ ATCA image (beam size of $8^{\prime \prime} \times 4^{\prime \prime}$ ). Green contours show the SUMSS radio emission at $843 \mathrm{MHz}$. The background image is the DSS blue image starting at $4 \mathrm{mJy} \mathrm{beam}^{-1}$ and are spaced in intervals increasing by a factor of 2. The red contours start at $5 \times \sigma_{r m s}$ and are spaced in intervals increasing by factor of 2 . 


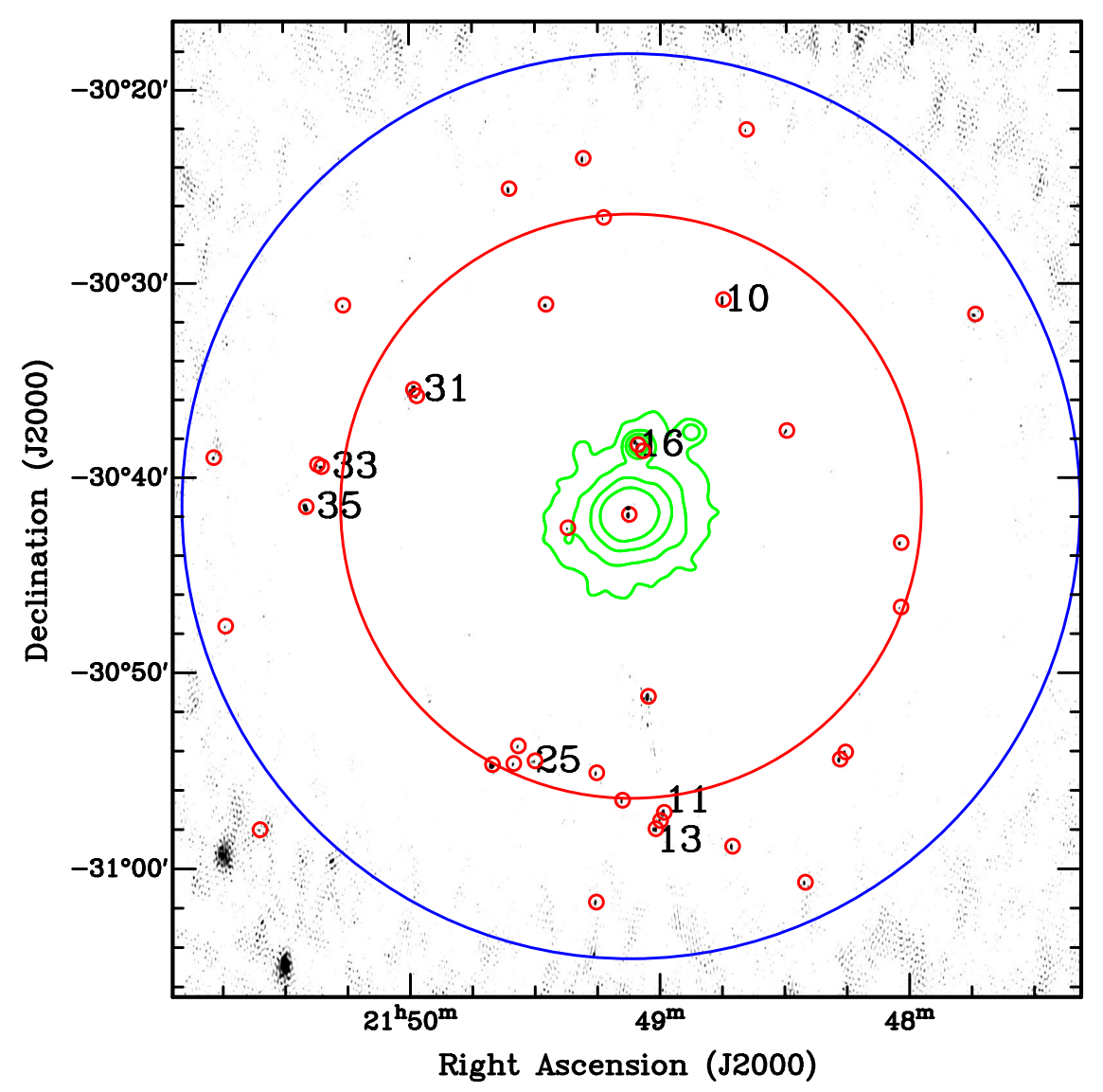

Figure 4.27: RXCJ2149.1-3041: Positions of the all detected radio sources and the radio ID of extended sources are annotated on the radio image $(1.4 \mathrm{GHz})$ with small red open circles. The $\mathrm{X}$-ray contours in green are also overlaid on the full resolution radio image. The blue circle represent the ATCA primary beam at $1.4 \mathrm{GHz}$ and the large red circle shows the $2 \mathrm{Mpc}$ radius around the cluster. 

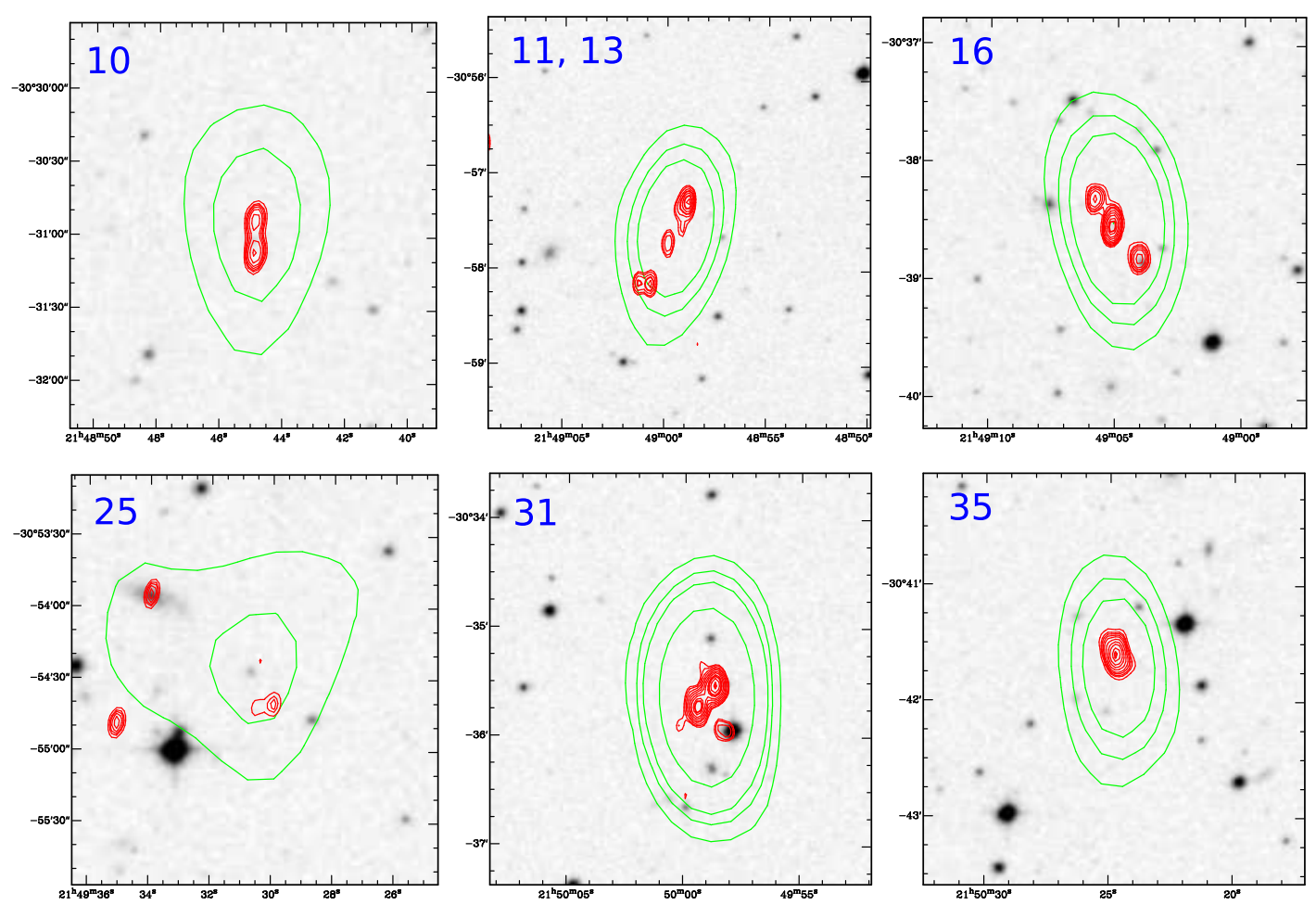

Figure 4.28: Postage stamps of extended sources in RXCJ2149.1-3041: Red contours demonstrate the radio emission of detected extended sources in the 1.4 GHz ATCA image (beam size of $\left.9^{\prime \prime} \times 4^{\prime \prime}\right)$. Green contours show the SUMSS radio emission at $843 \mathrm{MHz}$. The background image is the DSS blue image starting at $4 \mathrm{mJy} \mathrm{beam}^{-1}$ and are spaced in intervals increasing by a factor of 2. The red contours start at $4 \times \sigma_{r m s}$ and are spaced in intervals increasing by factor of 2 . 


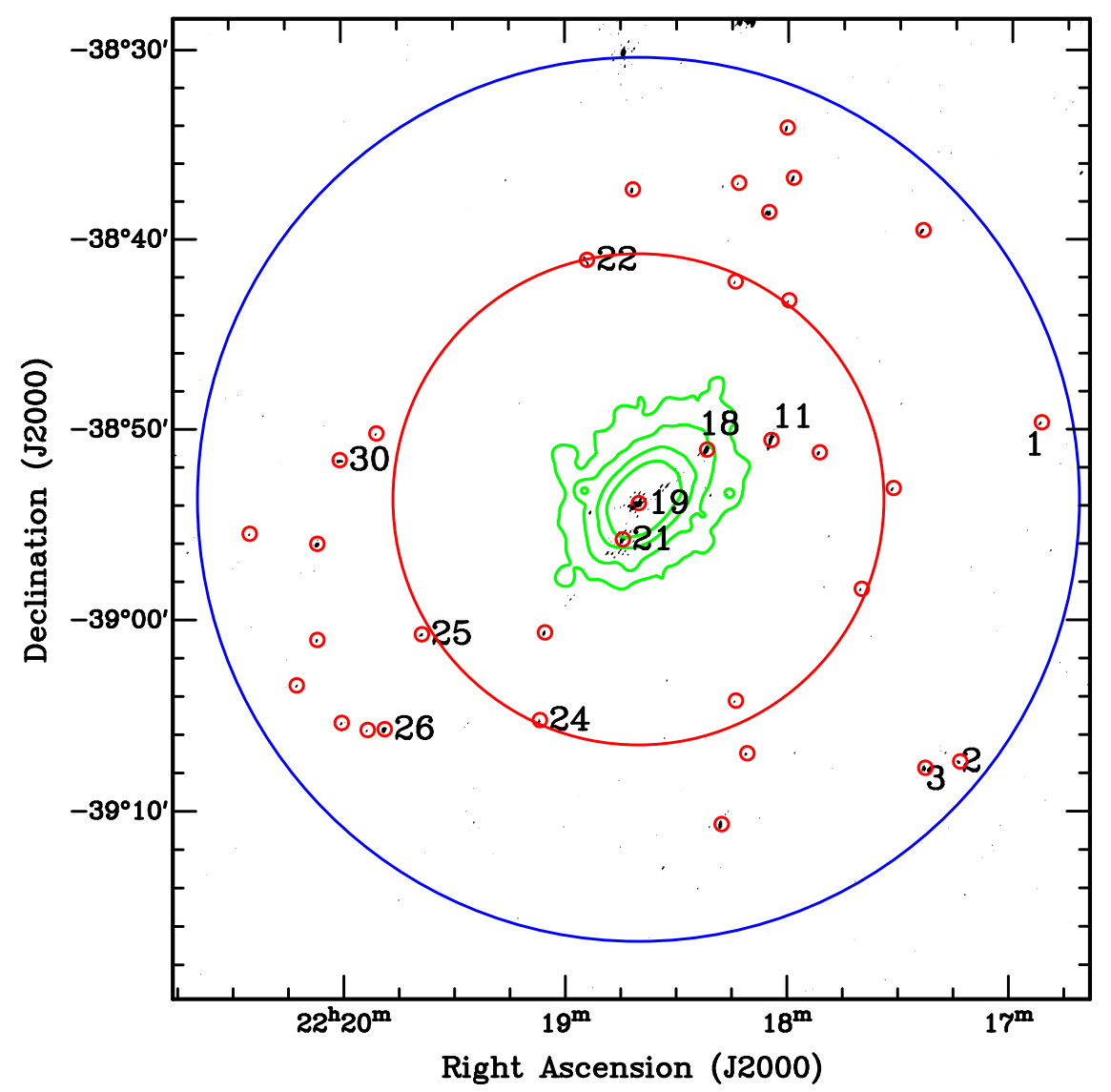

Figure 4.29: RXCJ2218.6-3853: Positions of the all detected radio sources and the radio ID of extended sources are annotated on the radio image $(1.4 \mathrm{GHz})$ with small red open circles. The $\mathrm{X}$-ray contours in green are also overlaid on the full resolution radio image. The blue circle represent the ATCA primary beam at $1.4 \mathrm{GHz}$ and the large red circle shows the $2 \mathrm{Mpc}$ radius around the cluster. 

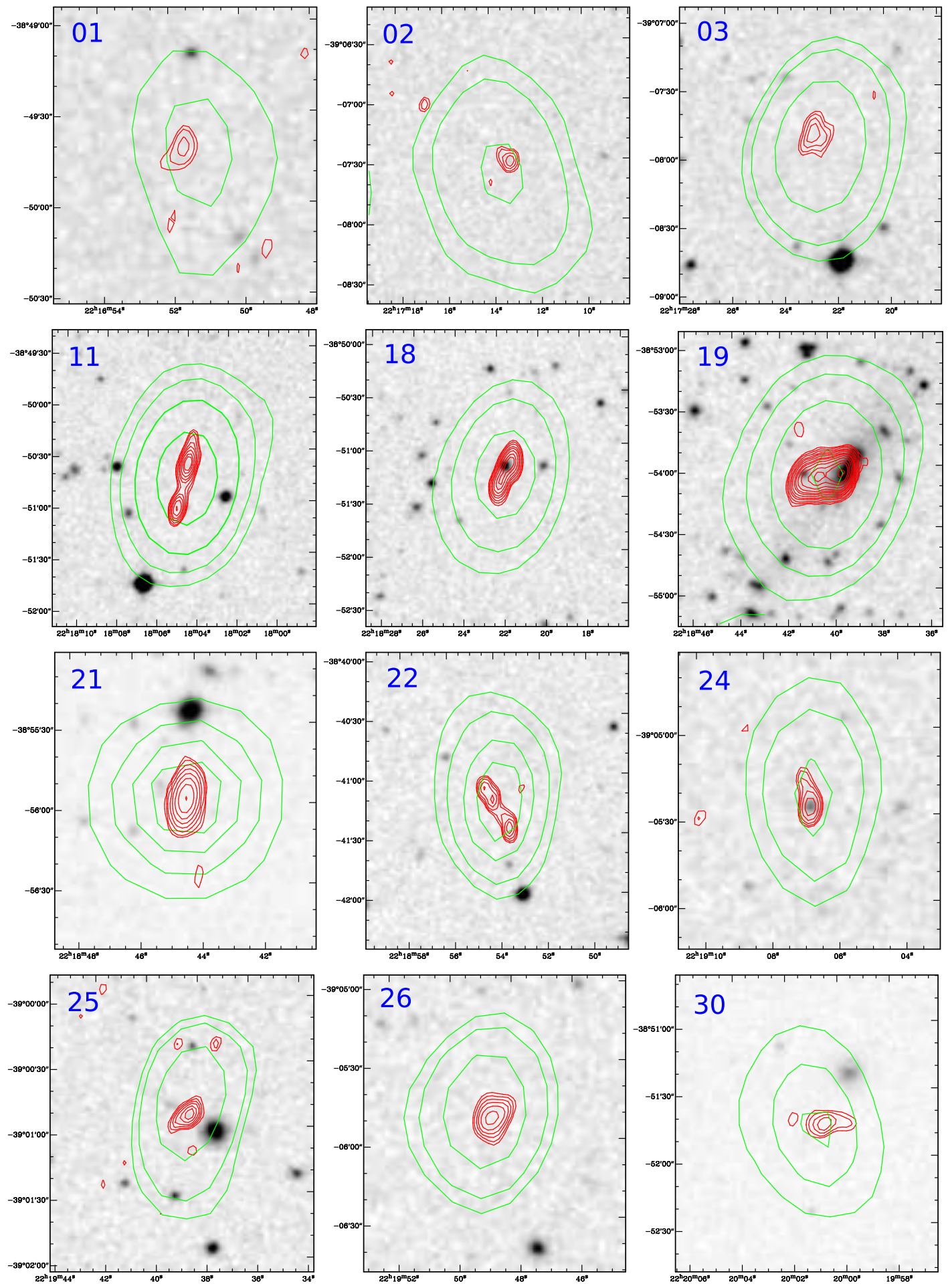

Figure 4.30: Postage stamps of extended sources in RXCJ2218.6-3853: red contours demonstrate the radio emission of detected extended sources in the $1.4 \mathrm{GHz}$ ATCA image (beam size of $8^{\prime \prime} \times 4^{\prime \prime}$ ) overlaid on the DSS blue image starting at $4 \mathrm{mJy} \mathrm{beam}^{-1}$ and are spaced in intervals increasing by a factor of 2 . Green contours show the SUMSS radio emission at $843 \mathrm{MHz}$. The red contours start at $4 \times \sigma_{\text {rms }}$ and are spaced in intervals increasing by factor of 2 . 


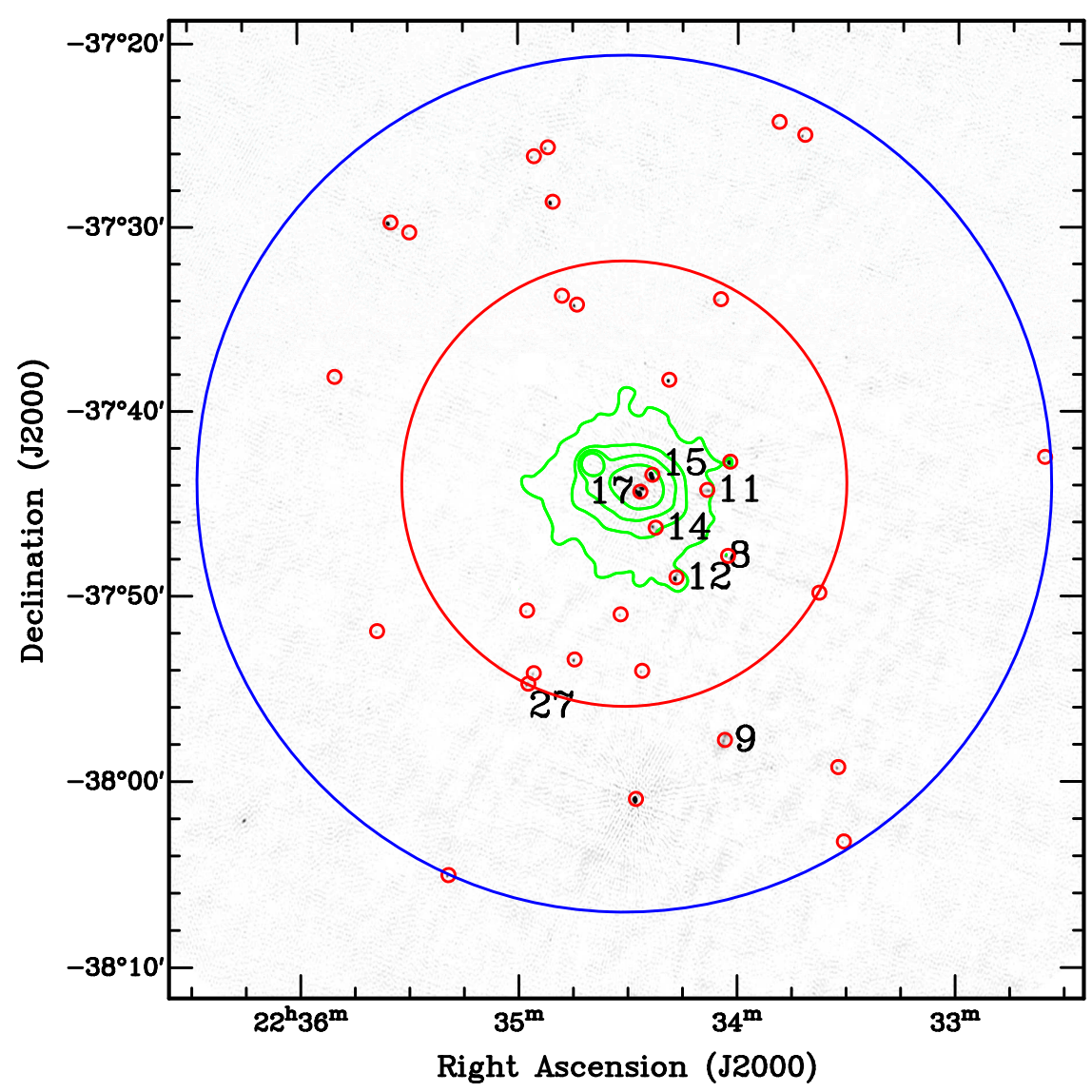

Figure 4.31: RXCJ2234.5-3744: Positions of the all detected radio sources and the radio IDs of extended sources are annotated on the radio image $(1.4 \mathrm{GHz})$ with small red open circles. The $\mathrm{X}$-ray contours in green are also overlaid on the full resolution radio image. The blue circle represent the ATCA primary beam at $1.4 \mathrm{GHz}$ and the large red circle shows the $2 \mathrm{Mpc}$ radius around the cluster. 

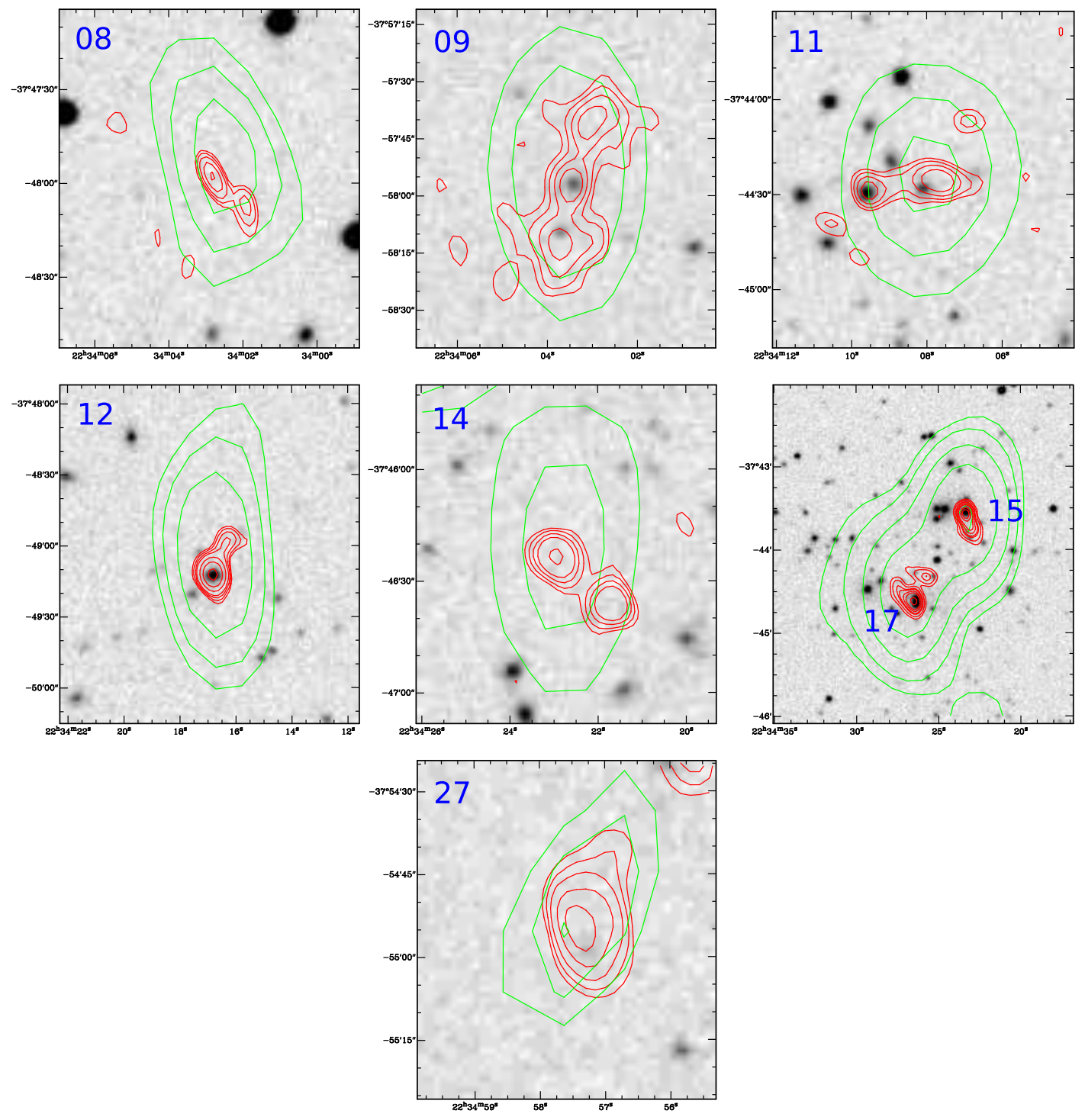

Figure 4.32: RXCJ2234.5-3744: red contours demonstrate the radio emission of detected extended sources in the $1.4 \mathrm{GHz}$ ATCA image (beam size of $8^{\prime \prime} \times 7^{\prime \prime}$ ). Green contours show the SUMSS radio emission at $843 \mathrm{MHz}$ overlaid on the DSS blue image starting at 4 mJy beam ${ }^{-1}$ and are spaced in intervals increasing by a factor of 2 . The red contours start at $4 \times \sigma_{r m s}$ and are spaced in intervals increasing by factor of 2 . 


\section{§5. Diffuse Radio Emissions in the REXCESS Clusters sub-sample}

In this chapter, details of the two detected diffuse radio emissions in clusters Abell 3888 and RXCJ0225.1-2928 are given. The integrated spectral index, cluster morphology and the thermal and non-thermal correlations of the detected radio halo in A3888 are presented. Possible explanations of the origin of the discovered peculiar diffuse emission in cluster RXCJ0225.1-2928 are also discussed. The remaining observed non-halo clusters are presented as a statistical sample in Chapter 7 . In this chapter we assume a $\Lambda \mathrm{CDM}$ cosmology with $\mathrm{H}_{0}=70 \mathrm{kms}^{-1} \mathrm{Mpc}^{-1}, \Omega_{m}=0.3$ and $\Omega_{\Lambda}=0.7$.

\subsection{The Radio Halo in Abell 3888}

In this section, we present the confirmation of the radio halo in Abell 3888 and discuss its observational properties. We also present different scaling relations between the clusters observables and the radio halo power which are proposed in the literature.

\section{§5.1.1. Radio Halo Properties in A3888}

We have created four low resolution, $300 \mathrm{MHz}$ sub-band images from the CABB dataset at central frequencies 1.4, 1.8, 2.1 and $2.4 \mathrm{GHz}$. Additionaly, we have made low resolution image using the combined pre-CABB and CABB (H214 and H168, $300 \mathrm{MHz}$ bandwidth) data at $1.4 \mathrm{GHz}$. Analysis of the low resolution image (combined pre-CABB and $\mathrm{CABB})$ at $1.4 \mathrm{GHz}\left(73^{\prime \prime} \times 58^{\prime \prime}\right.$, rms noise $\left.120 \mu \mathrm{Jy}\right)$ has confirmed a giant radio halo in the centre of A3888. In Figure 5.1, the halo emission in the low-resolution image made from the combined CABB $(\mathrm{H} 168+\mathrm{H} 214)$ and pre-CABB datasets (see Table 4.4 for details) is indicated with blue contours. Green contours represent the high resolu-

Table 5.1: Flux of the embedded radio sources in the halo region. The fluxes are in mJy.

\begin{tabular}{lcccc}
\hline \hline & $1.4 \mathrm{GHz}$ & $1.8 \mathrm{GHz}$ & $2.1 \mathrm{GHz}$ & $2.4 \mathrm{GHz}$ \\
\hline Total emission (low resolution) & 217 & 169.8 & 131 & 111 \\
HT1+HT2 (J223426-374432) & 83 & 70.6 & 53.34 & 43.7 \\
HT3 (J223423-374337) & 96.5 & 81.4 & 63.97 & 56.3 \\
Source 14 (J223422-374629) & 9.93 & - & - & - \\
Halo & 27.57 & 17.8 & 13.96 & 11 \\
\hline
\end{tabular}




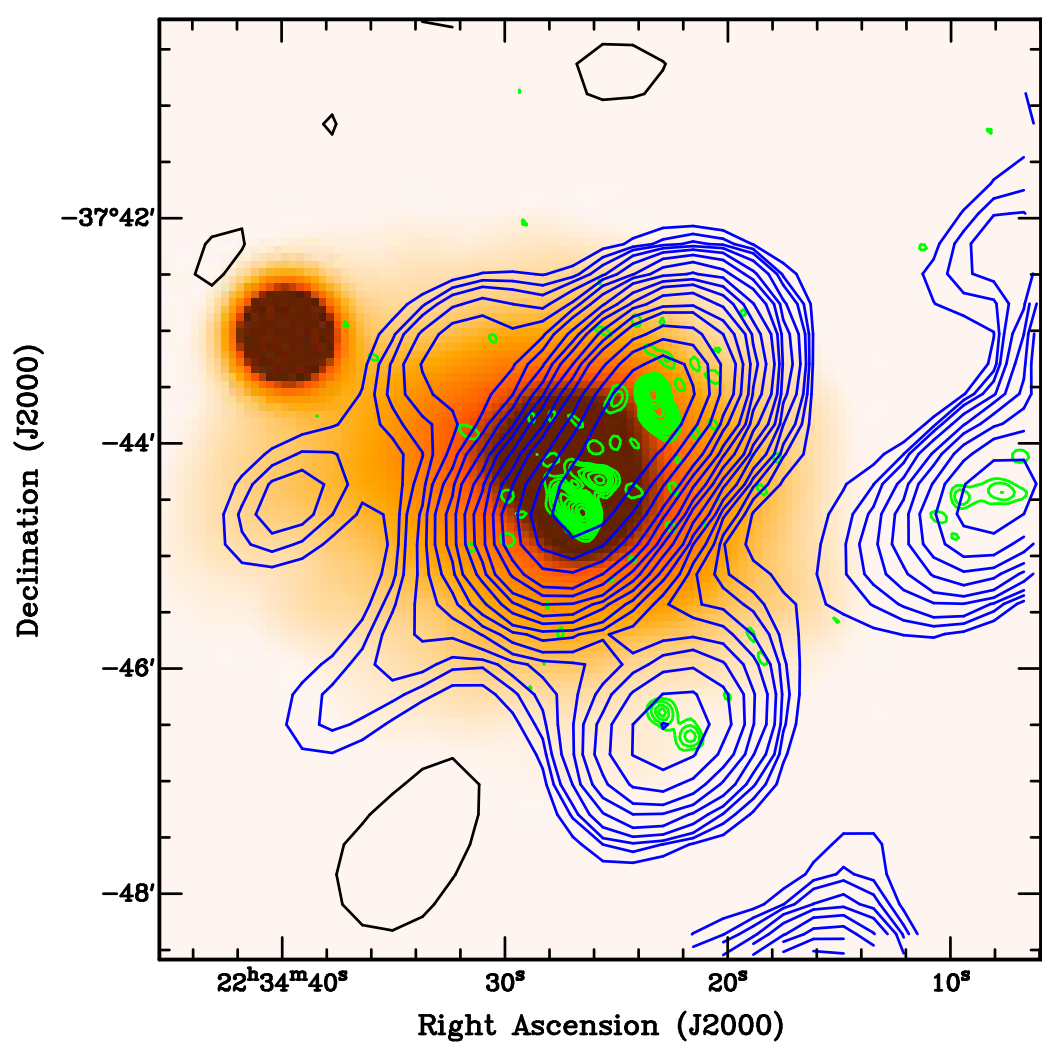

Figure 5.1: Radio halo in the centre of A3888. Blue contours represent the radio emission of the cluster in low resolution (see Table 4.4 for details) image. The contours start at $360 \mu \mathrm{Jy}_{\text {beam }}{ }^{-1}$ and are spaced by factor of $\sqrt{2}$ intervals in brightness. Black contour with value of $-360 \mu \mathrm{Jy}$ beam $^{-1}$ shows negative regions in the image. Green contours start at $300 \mu \mathrm{Jy}_{\text {beam }}{ }^{-1}$ and are spaced by factor of $\sqrt{2}$ intervals in brightness and represent the radio emission of the cluster in high resolution image. The X-ray image is the background image.

tion made from $\mathrm{CABB}$ data in the $6 \mathrm{~A}$ config. The contours are overlaid on the XMMNewton X-ray data of A3888. It can be easily realised that the radio halo has a NW-SE orientation. The presence of three HT galaxies inside the radio halo region is evident in the high resolution image made from the CABB (6A) data. We attempted to peel all the contaminating radio sources inside the halo region. We used different weightings and robust parameters, though, we encountered cases in which the extended emissions of the HT galaxies were not removed completely or we over subtracted the halo emission. To measure the halo flux, we have created a Stokes I image at low resolution (HT galaxies + halo) and a high resolution image (HT galaxies) from all the combined CABB data in each of the four CABB sub-bands. We have measured the flux of the contaminating radio sources (in the halo region) in the high resolution images (in which the halo is resolved out) and then we have subtracted the flux of the contaminating HT galaxies from the total flux measured in the low resolution images. Details of the embedded HT galaxies and other contaminating source are given in Table 5.1. Two of the HTs were un-resolved (J223426-374432, see Appendix Table A.15) at $1.4 \mathrm{GHz}$ but were well-resolved at 1.8, 2.1 and $2.4 \mathrm{GHz}$, thus we have reported the sum flux of the two 
aforementioned HTs in Table 5.1.

The flux error of the halo was estimated as $\Delta S_{H}=\sqrt{\left(S_{H} \times \Delta S_{\text {err,cal }}\right)^{2}+\left(\sigma_{\text {rms }} \times \sqrt{N_{\text {beam }}}\right)^{2}}$

where $\mathrm{S}_{H}$ is the radio halo flux and $S_{\text {err,cal }}$ is the typical calibration error for the ATCA which is about $10 \%$ of the total measured flux. $\mathrm{N}_{\text {beam }}$ is the number of beams which fills the radio halo area. The total flux of the radio halo was measured as $27.57 \pm 3.13 \mathrm{mJy}$ at 1.4 GHz. We have calculated the halo flux in the remaining sub-bands to be $17.8,13.69$ and $11 \mathrm{mJy}$ at $1.8,2.1$ and $2.4 \mathrm{GHz}$, respectively.

The largest angular scale (LAS) of the halo was measured as $6.23^{\prime}$. Given that the angular scale of the cluster is $6.26^{\prime} \mathrm{Mpc}^{-1}$ at the redshift of the cluster $(\mathrm{z}=0.153)$, the measured LAS of the halo corresponds to the largest linear scale (LLS) of $996 \mathrm{kpc}$. The smallest linear scale (SLS) was $547 \mathrm{kpc}$. The measurement of LLS was based on the measurements of the area enclosed in the contour level greater than the $3 \times \sigma_{r m s}$ beam $^{-1}$.

\section{$\S$ 5.1.2. Integrated Spectral Index}

As we mentioned earlier, the largest angular extent of the halo was $6.23^{\prime}$ at $1.4 \mathrm{GHz}$. Since the shortest baseline in our observations is $31 \mathrm{~m}$, measuring the total flux of the radio halo was possible across the entire $2 \mathrm{GHz}$ bandwidth. To measure the integrated spectral index of the halo, we have used all four sub-band images. Figure 5.2 shows the spectrum of the radio halo in A3888 which was obtained by fitting to the four data points across the CABB band. The black dots show the measured integrated flux at different frequencies and the solid red line shows the fit to the data points. The linear fit in log-scale yields a spectral index of $\alpha=1.48 \pm 0.14$. The flux errors were used as a weighting to the data points in the fitting procedure. The K-corrected power was $1.89 \times$ $10^{24} \mathrm{~W} \mathrm{~Hz}^{-1}$ at $1.4 \mathrm{GHz}$ using equation below. The convention of $\mathrm{s} \propto v^{-\alpha}$ is assumed.

$$
P_{1.4 G H z}=\frac{4 \pi D_{L}^{2}(z)}{(1+z)^{1-\alpha}} S_{1.4 G H z}
$$

where $D_{L}$ is the luminosity distance. All the properties of the radio halo in A3888 are given in Table 5.2. In the following, we describe currently proposed scaling relations for halos and compare the properties of the halo in A3888 with the predicted values from the published correlations.

\section{$\S$ 5.1.3. Power/Size Scaling Relation}

We first consider the relationship between the power at $1.4 \mathrm{GHz}$ and the largest linear extent for all known radio halos to date. In Figure 5.3 we have marked our radio halo with filled red dot alongside the 61 known radio halos from the literature. The location 


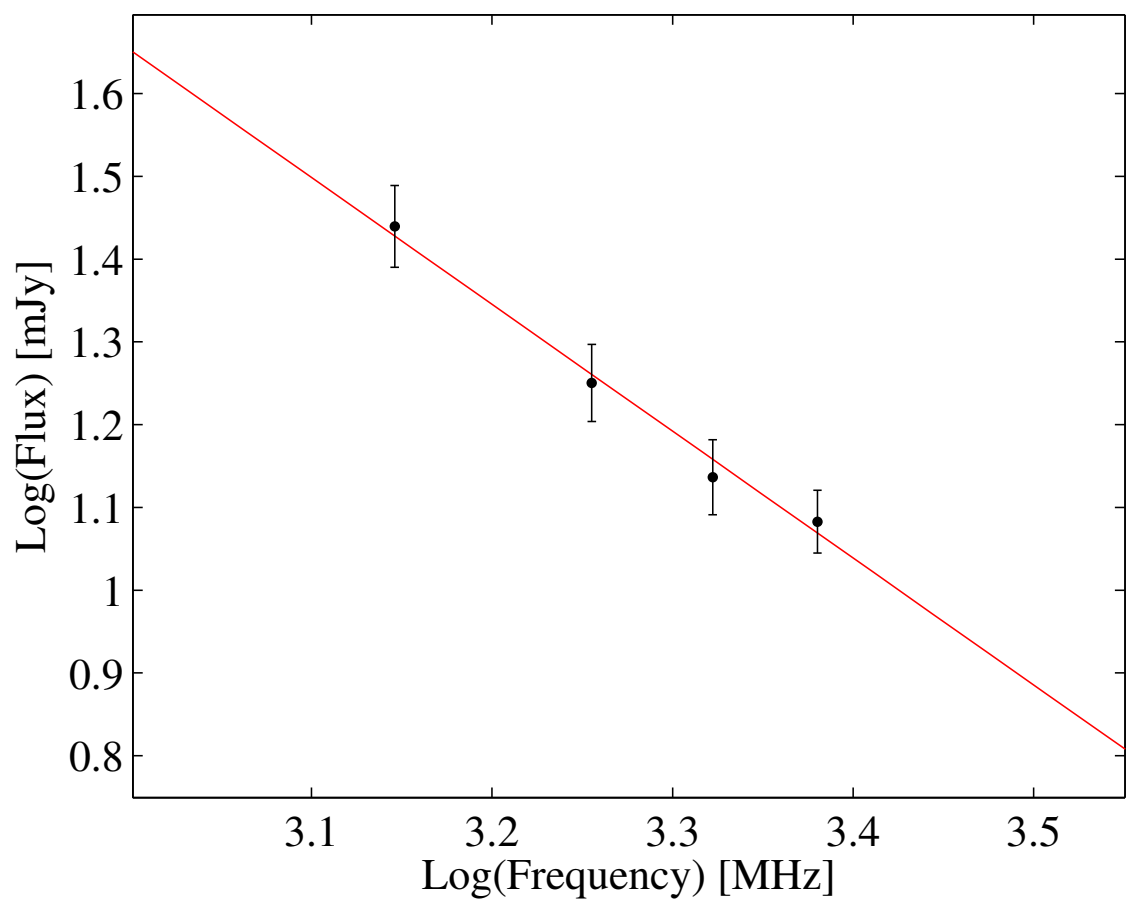

Figure 5.2: The spectrum of the radio halo in A3888 obtained acroos the CABB band. The black circles show the measured integrated flux at different frequencies across the CABB band and the solid red line shows the fit to the data points. The linear fit to all of the flux values in log-scale yields the spectral index $\alpha=1.48 \pm 0.14\left(s_{V} \propto v^{-\alpha}\right)$.

of A3888 is consistent with the majority of the halo observations. Cassano et al. (2007) introduced a new term, "effective radio halo radius $\mathrm{R}_{H}$ " where $\mathrm{R}_{H}$ is given by $\mathrm{R}_{H}=$ $\frac{1}{2} \sqrt{L_{m a j} \times L_{m i n}} . \mathrm{L}_{m a j}$ and $\mathrm{L}_{\text {min }}$ are the major and minor width of the radio halo, respectively. This parameter is calculated by measuring the major and minor length of the halo at $3 \sigma$ level. Cassano et al. (2007) claim that this correlation is an intrinsic property of radio halos rather than a false impression dictated by the detection limits and hence is unaffected by different noise levels in radio images. Indeed, radio halos have low surface brightness and it is possible that when they are less powerful, they are partially detected and the edges of the halo could be buried under the noise level of the radio image. However, Cassano et al. (2007) mentioned that they have noticed that less powerful halos were detected in the images with a higher level of sensitivity in their survey. They also have investigated the radio profiles of the detected radio halos in their cluster sample. They only considered the radio halos that were not affected by contaminated extended emissions and they reported that with the increasing halo-centric radius, the brightness profile reaches a plateau. Thus, they proposed that this feature could be used as a proxy for the halo size measurement in radio maps. It is known that the size of radio halos calculated by using the LLS or $\mathrm{R}_{H}$ are both affected by the sensitivity of the radio maps. In a comparative analysis of radio halos Murgia et al. (2009) suggested to 
Table 5.2: Properties of the radio halo in A3888.

\begin{tabular}{ll}
\hline \hline $\mathrm{S}_{1.4}$ & $27.57 \pm 3.13 \mathrm{mJy}$ \\
$\mathrm{P}_{1.4}$ & $1.89 \pm 0.22 \times 10^{24} \mathrm{~W} \mathrm{~Hz}^{-1}$ \\
Spectral index & $1.48 \pm 0.14$ \\
LAS $\times$ SAS & $6.23^{\prime} \pm 0.18^{\prime} \times 3.42^{\prime} \pm 0.11^{\prime}$ \\
$\mathrm{LLS} \times \mathrm{SLS}$ & $996 \pm 30 \mathrm{kpc} \times 547 \pm 17 \mathrm{kpc}$ \\
$\mathrm{L}_{X[0.1-2.4]}$ & $6.64 \pm 0.0025 \times 10^{44} \mathrm{erg} / \mathrm{s}$ \\
$\mathrm{Y}_{s z}$ & $8.7 \pm 2.32 \times 10^{-5} \mathrm{Mpc}^{2}$ \\
$\mathrm{M}_{500}$ & $7.19 \pm 0.26 \times 10^{14} \mathrm{M}_{\odot}$ \\
\hline
\end{tabular}

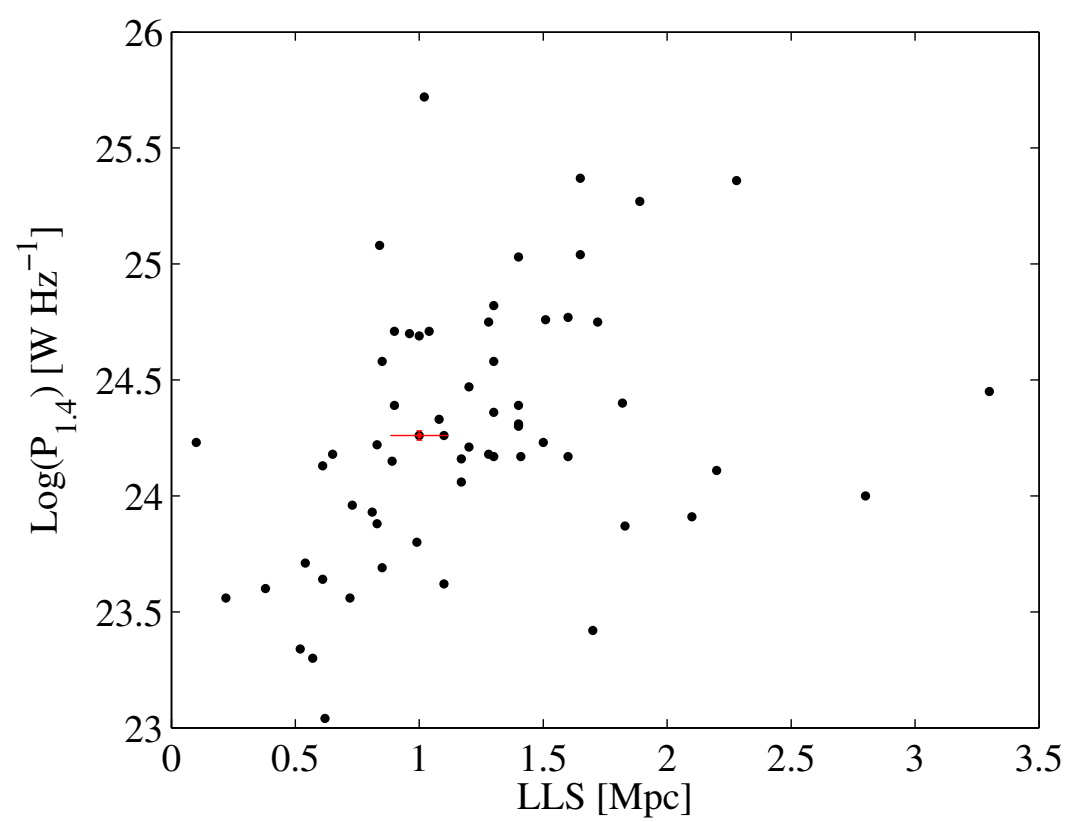

Figure 5.3: $\mathrm{P}_{1.4}$ vs LLS of radio halos from the literature. A3888 is shown with a red filled dot and the black dots are the 61 halos from the literature. We could not find the LLS errors for the majority of the halos in the literature and we did not include the error bars for the halos in the literature.

measured the size of the radio halos by fitting to their azimuthally-averaged brightness profile with an exponential law, obtaining the e-folding radius. They showed that the e-folding radius is independent of the sensitivity of the radio images and therefore can be used to define the linear size of the diffuse sources. The location of our radio halo is consistent with the observed correlation between the halo power and LLS of the radio halos from the literature. Since the noise level of our low resolution radio map is comparable with the $\sigma_{r m s}$ of the images have been used to extract the $\mathrm{R}_{H}$ in the literature, in Figure 5.4, we plot our radio halo alongside the other radio halos detected in the literature. Again, the location of our radio halo is consistent with the predicted location by the correlation. The power of the halo in A3888 is consistent with the $\mathrm{P}_{1.4} / \mathrm{L}_{X}$ correlation proposed by Cassano et al. (2006). This correlation will be discussed later in 


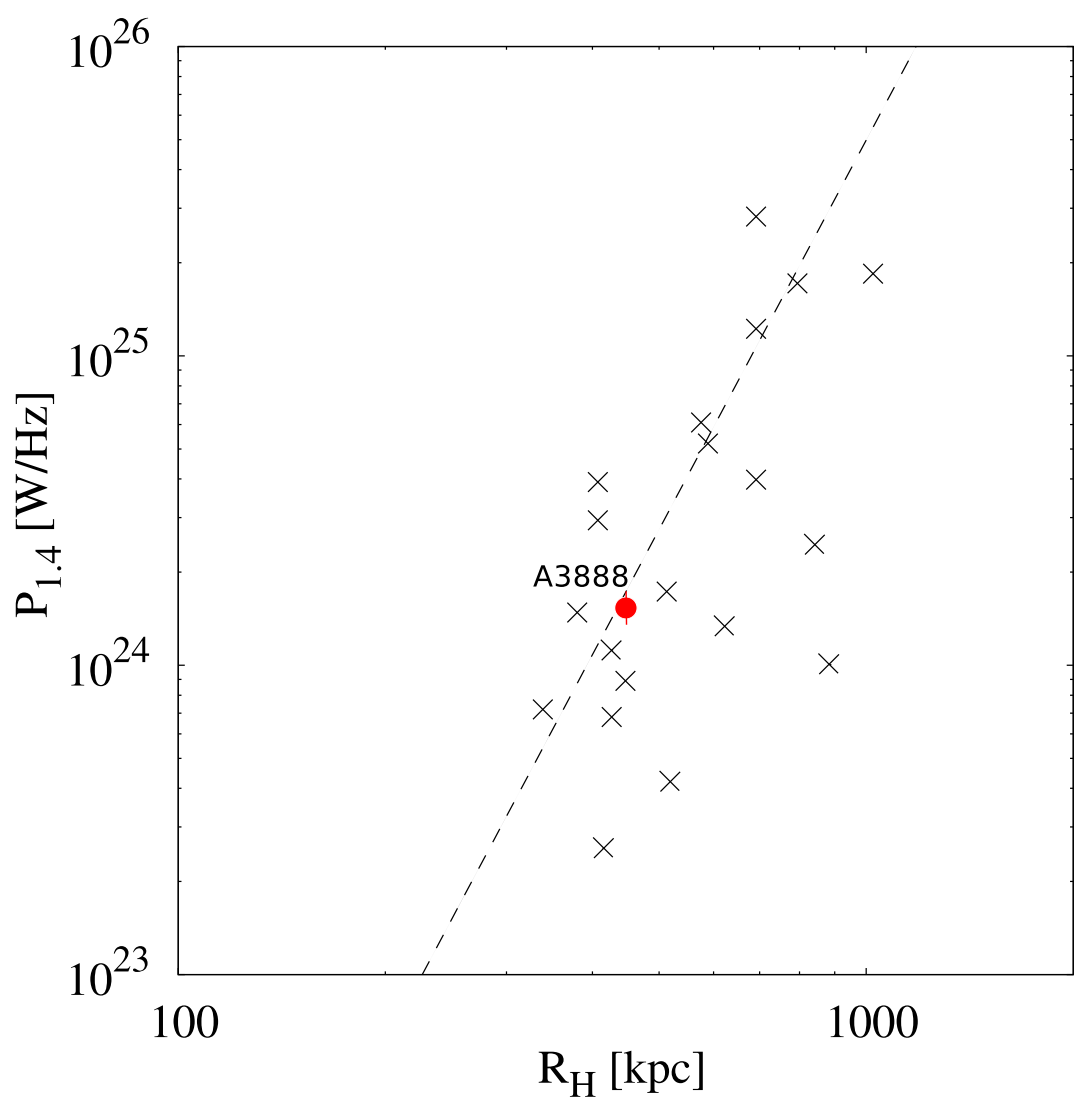

Figure 5.4: $\mathrm{P}_{1.4}$ vs $\mathrm{R}_{H}$. A3888 is shown with red filled circle and other radio halos from the literature are shown with Xs. The image is adapted from Farnsworth et al. (2013) and has been modified.

Chapter 7.

\section{§5.1.4. Morphology of A3888: Literature Review}

Despite the lack of optical analysis of A3888 in the literature, this cluster has been deeply observed with the XMM-Newton (Böhringer et al., 2007) and its X-ray properties have been reported in several publications. For instance, Pratt et al. (2009) measured the X-ray luminosity of the cluster and pointed out that A3888 is an X-ray luminous cluster with $\mathrm{L}_{X}=6.363 \times 10^{44} \mathrm{erg} \mathrm{s}^{-1}[0.1-2.4 \mathrm{keV}]$ which indicates this is a massive cluster $\left(\mathrm{M}_{500}=7.36 \times 10^{14} M_{\odot}\right)$. Meanwhile, an X-ray sub-structure analysis of A3888 has been carried out in multiple papers; Pratt et al. (2009) used the traditional threshold of $w=0.01$ as the morphology discriminator where clusters with centroid shift $w<0.01$ are considered relaxed while clusters with $w>0.01$ are classified as morphologically disturbed. Soon after, Böhringer et al. (2010) reported that the centroid shift parameter in the $R_{500}$ aperture is 0.0075 , however, the third order power ratio $\mathrm{p}_{3} / \mathrm{p}_{0}$ (see $\mathrm{C}$ for details) was reported as a negative value. However, according to the threshold used by Pratt et al. (2009), A3888 is a regular cluster. In some cases, X-ray observations with low photon counts are affected by noise which produce artificial structures (bias). After 
noise corrections and bias subtraction, the third order power ratio $\mathrm{p}_{3} / \mathrm{p}_{0}$ might become negative which is considered as zero (Böhringer et al., 2010).

Later on, Chon et al. (2012) revised the centroid shift and power ratio calculations of A3888 and used the mean centroid value of all apertures as the reference centre. The variance of $w$ and the power ratios depend on the choice of the reference centre. By using this new reference centre they obtained a smaller $w$ and a more reliable $\mathrm{p}_{3} / \mathrm{p}_{0}$ value for A3888. Chon et al. (2012) also used a new threshold for the morphology discriminators where disturbed clusters have centroid shift with $w>0.006$ or the third order power P3 $>2 \times 10^{7}$. Chon et al. (2012) reported the values for $w$ and P3 to be 0.00854 and $0.8 \times 10^{7}$, respectively for A3888 and classified A3888 as a disturbed cluster.

Soon after, Weißmann et al. (2013) used a new morphology estimator to identify the disturbed/relaxed clusters. They measured the third power ratio $\mathrm{p}_{3} / \mathrm{p}_{0}$ in $0.3-1 \mathrm{R}_{500}$ aperture. They pointed out that A3888 is an intermediate cluster in terms of substructure with some local asymmetries confined to the core but with the global properties consistent with a relaxed system (private communication with Weißmann et al., 2013).

The morphology analysis of this cluster was also corroborated with the available optical pieces of evidence in the literature. A3888 has no central cD galaxy (Haarsma et al., 2009), however, it is consists of three brightest cluster galaxies (BCGs) and the one in the middle of the BCG group is located $112 \mathrm{kpc}$ away from the X-ray peak position (Haarsma et al., 2010). The large BCG offset from the X-ray peak and the lack of $\mathrm{cD}$ galaxy are often indicative of dynamical interactions in a young cluster. To provide a more plausible interpretation for the available combined X-ray and optical evidence for A3888 mentioned in above, we have considered the work presented by Mann and Ebeling (2012). They scrutinised a sample of 108 most X-ray luminous clusters in the redshift range $0.15 \leq \mathrm{z} \leq 0.7$ with available Chandra X-ray observation. They found that clusters with the BCG/X-ray peak separation larger than $42 \mathrm{kpc}$ with distinct multiple or small substructures in the X-ray brightness distribution are undoubtedly in active merger. In the next chapter, we present our new optical observation of A3888 and our optical substructure test to provide a more clear insight into the morphology of the cluster.

Here we re-confirm the existence of a GRH in A3888 which is a massive and dynamically active cluster. The location of A3888 is in a agreement with the $\mathrm{P}_{1.4} / \mathrm{L}_{X}$, $\mathrm{P}_{1.4} / \mathrm{Y}_{S Z}, \mathrm{P}_{1.4} / \mathrm{M}_{500}$ and $\mathrm{P}_{1.4} /$ LLS correlations proposed by Cassano et al. (2006). These results are presented in Chapter 7. 


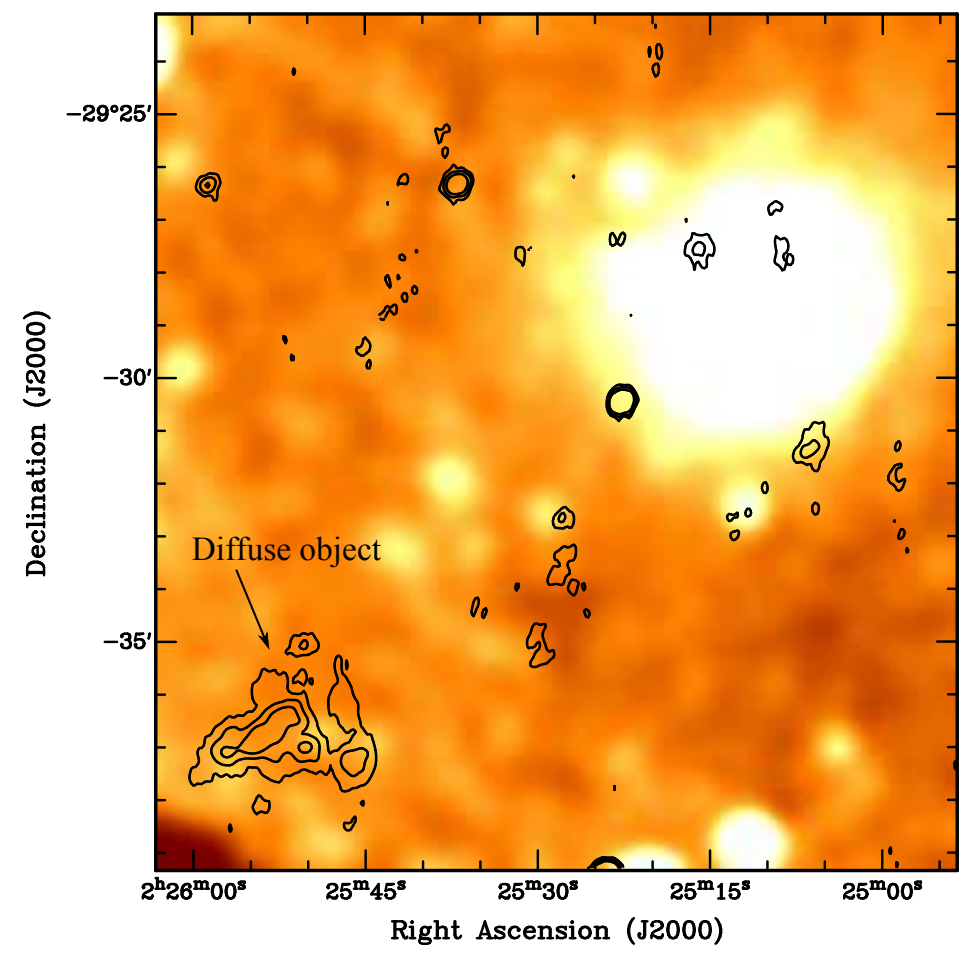

Figure 5.5: The high resolution image (pre-CABB data) of the diffuse radio emission in the cluster RXCJ0225.1-2928 is shown with the black contours. The contours start at $260 \mu \mathrm{Jy}$ beam $^{-1}$ and are spaced by 2 times intervals. The background is the X-ray image of the cluster.

\section{§5.2. The Peculiar Diffuse Source in the Cluster RXCJ0225.1- 2928}

Radio observations of the cluster RXCJ0225.1-2928 were carried out at 1.344 and 1.432 GHz with the ATCA in EW352 and 750B configurations in June and July 2007, respectively. Details of the ATCA observation of the cluster RXCJ0225.1-2928 are given in Table 4.1. The minimum baseline length of $31 \mathrm{~m}$ to maximum baseline length of $4.5 \mathrm{~km}$ provides the angular sensitivity to the scales between $12^{\prime \prime}$ to $24^{\prime}$ at $1.4 \mathrm{GHz}$. Figure 5.5 shows the discovered diffuse object (black contours) overlaid on the X-ray image of the cluster.

\section{§5.2.1. Stokes I and Polarisation Imaging}

Investigation of the full and low resolution radio images of the cluster RXCJ0225.12928 revealed that there is diffuse radio emission located $13^{\prime}(\sim 1 \mathrm{Mpc})$ away from the cluster centre. Details of the mentioned images are given in Table 5.3. Further analysis of the high resolution image which was created merely including baselines sensitive to spatial frequencies greater than $18.75 \mathrm{k} \lambda$ (baselines longer than $3750 \mathrm{~m}$, antenna 6 only) at $1.4 \mathrm{GHz}$ indicated that there is no core or hot spot in the region associated 
Table 5.3: Details of Stokes I and polarisation images created at $1.4 \mathrm{GHz}$ and are shown in Figures 5.7 and 5.5 or were used in the spectral index analysis.

\begin{tabular}{lccc}
\hline \hline $\begin{array}{l}\text { Freq } \\
\text { MHz }\end{array}$ & $\begin{array}{c}\text { FWHM, PA } \\
{ }^{\prime \prime} \times{ }^{\prime \prime},\end{array}$ & $\begin{array}{c}\sigma_{\text {rms }} \\
\text { mJy beam }^{-1}\end{array}$ & Notes \\
\hline 1400 & $66 \times 46,2$ & 0.124 & Figure 5.7 \\
1400 & $10 \times 4,-2$ & 0.083 & Figure 5.5 \\
1400 & $9 \times 6,-3$ & 0.070 & Stokes I, baselines $>3750 \mathrm{~m}$, antenna 6 only \\
1400 & $19 \times 16,-50$ & 0.045 & Figure 5.6 \\
178 & $259 \times 167,-6$ & 30 & MWA commissioning Stokes I \\
\hline
\end{tabular}

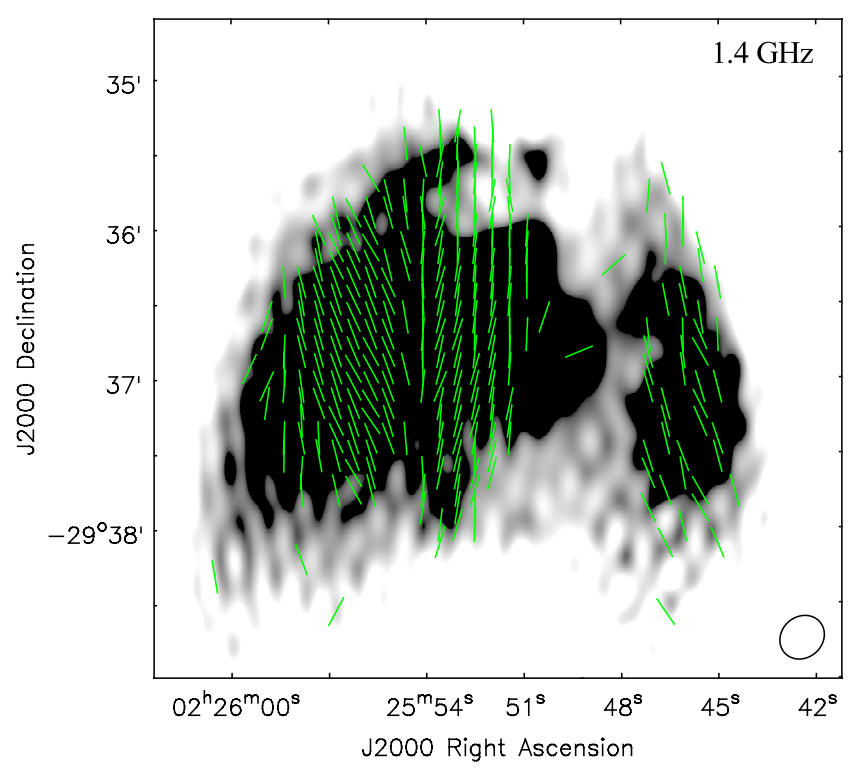

Figure 5.6: Magnetic field direction map of the diffuse radio source in RXCJ0225.1-2928. There are three well separated components in the polarisation map. The green sticks represent the orientation of the magnetic field across the detected diffuse source. The Stokes I image is the background.

with this radio object. The LAS of the source is $3.9^{\prime}$ (the physical units associated with this measurement are given later in $\S 5.2 .3$ ) and the measured flux at $1.4 \mathrm{GHz}$ is 37 mJy. We also have investigated the polarisation of the detected diffuse radio source. The polarisation and polarisation angle maps were created and the magnetic field direction of the radio source was determined via rotating the polarisation angle by $90^{\circ}$. The magnetic field direction is shown in Figure 5.6 (see Table 5.3 for details). The Polarisation angle pixels are masked in the case that the polarisation angle error was greater than $10^{\circ}$ or the Stokes I and polarisation intensity were less than $3 \times \sigma_{\text {rms }}$. Figure 5.6 shows that the polarisation of the source has three distinct components. It should be noted that the derived polarisation angle was not corrected for the rotation measure. 

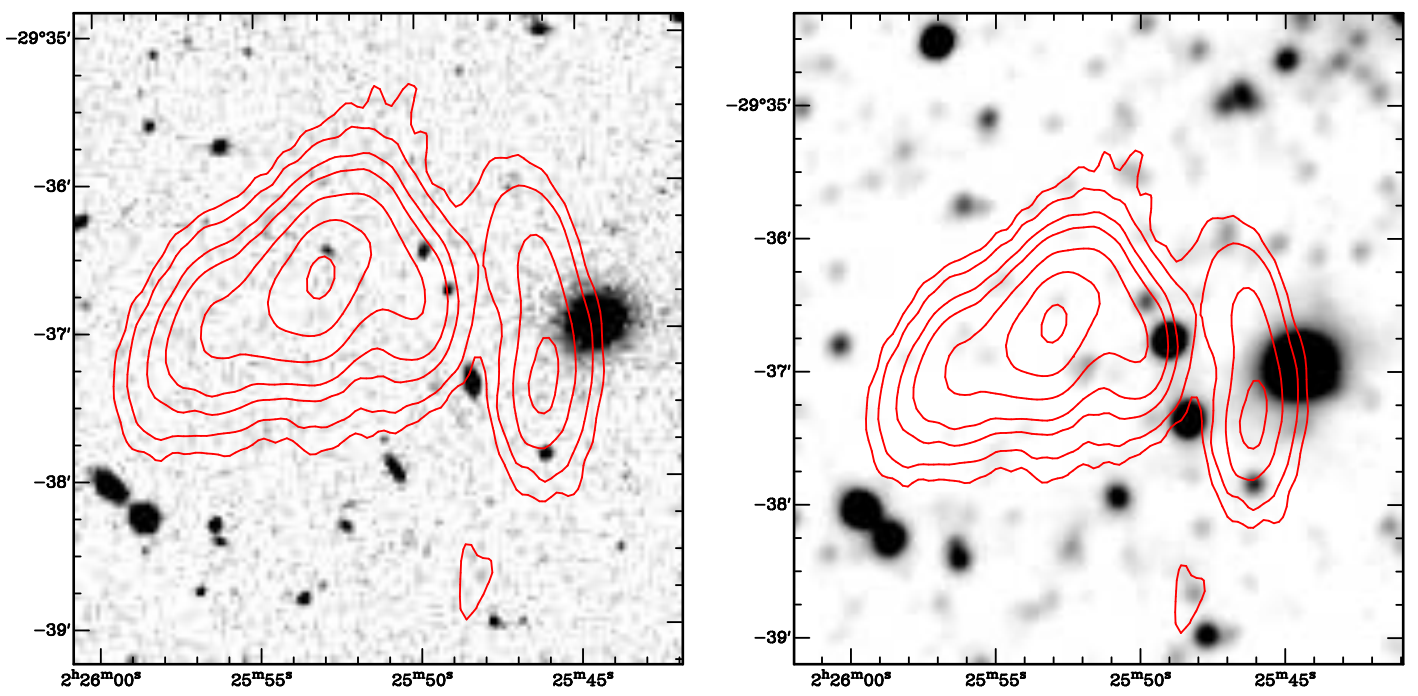

Figure 5.7: Radio emission from the low resolution images of the diffuse radio source in cluster RXCJ0225.1-2928 is indicated with the red contours. The contour levels are at $\sqrt{1,2,4,8, \ldots} \times$ $4 \times 124 \mu \mathrm{Jy}_{\text {beam }^{-1}}$. The background images are the r-band DSS2 (UK Schmidt Telescope Red (IIIaF) second epoch) and the WISE infra-red $(3.4 \mu \mathrm{m})$ from left to right, respectively.

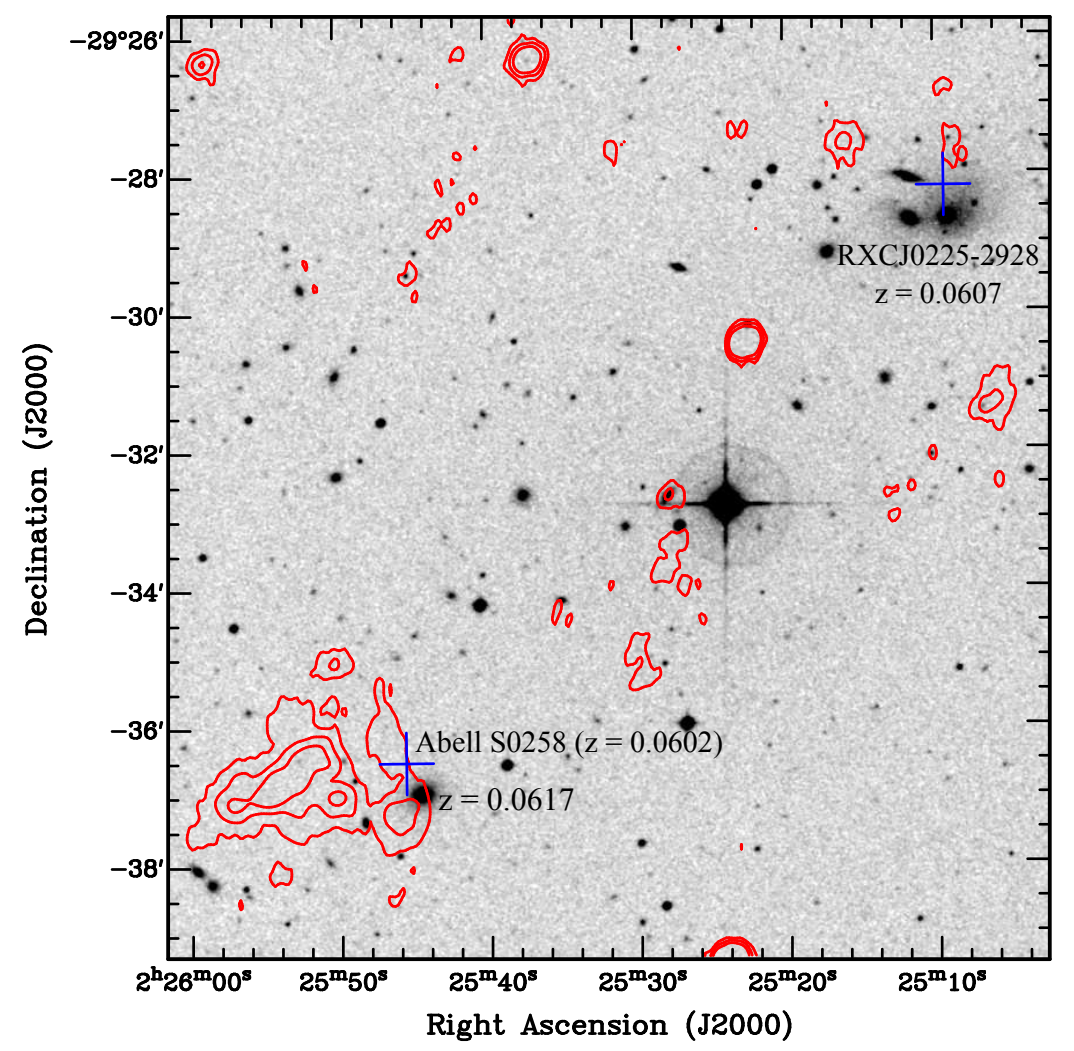

Figure 5.8: Positions of AS0258 (according to the NED) and RXCJ0225-2829 are shown with blue + . The red contour demonstrates the radio emission in the high resolution image. The background is the DSS2 red image. The lack of mass concentration around AS0258 is evident.

\subsubsection{Optical/IR Investigation of Cluster RXCJ0225.1-2928}

There is a prominent elliptical galaxy (Loveday, 1996) with redshift of the 0.0617 (Jones et al., 2009) in the west edge of the radio emission with optical ID 2MASX J02254441- 


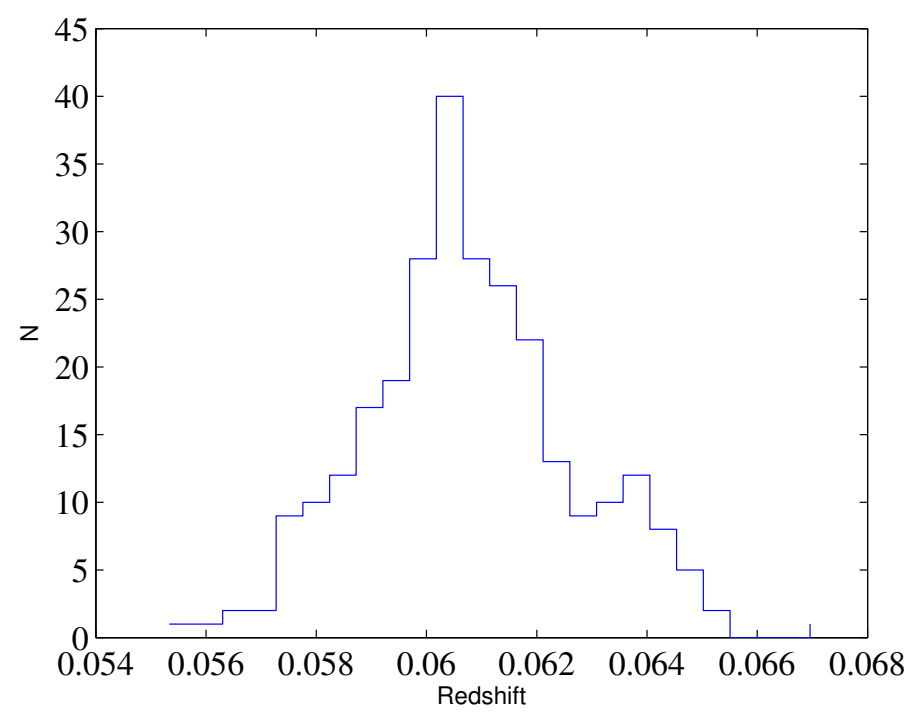

Figure 5.9: The redshift histogram of available redshifts in two degrees radius around the cluster RXCJ0225.1-2928 using the bin-width of $350 \mathrm{~km} \mathrm{~s}^{-1}$ in range of 0.055-0.068. A departure of Gaussianity in the histogram is evident at redshift of $\sim 0.064$.

2936567. However, there is no optical/IR counterpart directly associated with the detected diffuse radio emission. The lack of satellite galaxies around the elliptical galaxy in Figure 5.7 (both plots) is explicitly suggesting that there is no galaxy cluster in this region, and the elliptical galaxy is possibly a member of the cluster RXCJ0225.1-2928. The examination of the DSS2 r-band image and the NASA Wide-field Infra-red Survey Explorer (WISE) images in 3.4-22 $\mu \mathrm{m}$ revealed that there is no galaxy associated with the detected diffuse emission. In fact, all the Infra-red emitters in this region have optical IDs. In Figure 5.7 the DSS2 red and WISE Infra-red $3.4 \mu \mathrm{m}$ images are demonstrated from left to right, respectively. Abell et al. (1989) have reported that there is a galaxy cluster, Abell S0258 with redshift of 0.0602 which is located $11.36^{\prime}(918 \mathrm{kpc}$ ) far from the cluster RXCJ0225.1-2928 ( $\mathrm{z}=0.0607)$. Figure 5.8 shows the positions of the clusters RXCJ0225.1-2918 and A0S258 around the diffuse radio emission. We searched the NED within a two degrees radius around RXCJ0225.1-2928 to find the available spectroscopic redshifts from the literature. There are precisely 1488 spectroscopic redshifts in the range 0.0026-0.5274. We performed a simple cluster membership selection based on the redshift histogram with a bin-width of $350 \mathrm{~km} \mathrm{~s}^{-1}$ to determine the initial members of RXCJ0225.1-2928. Accordingly, we found that this cluster had 277 possible members in redshift range $0.054-0.068$. Figure 5.9 shows the redshift histogram of the members of the cluster RXCJ0225.1-2928. The departure from Gaussianity is evident at $\sim 0.064$. The distribution of galaxies associated with the second peak in the histogram (Figure 5.10, green stars) is quite sporadic and is not indicative of existence of any galaxy group or galaxy cluster. The contour plot associated with the possible member galaxies is shown in Figure 5.10 and suggest that there is a filamentary structure in a two 


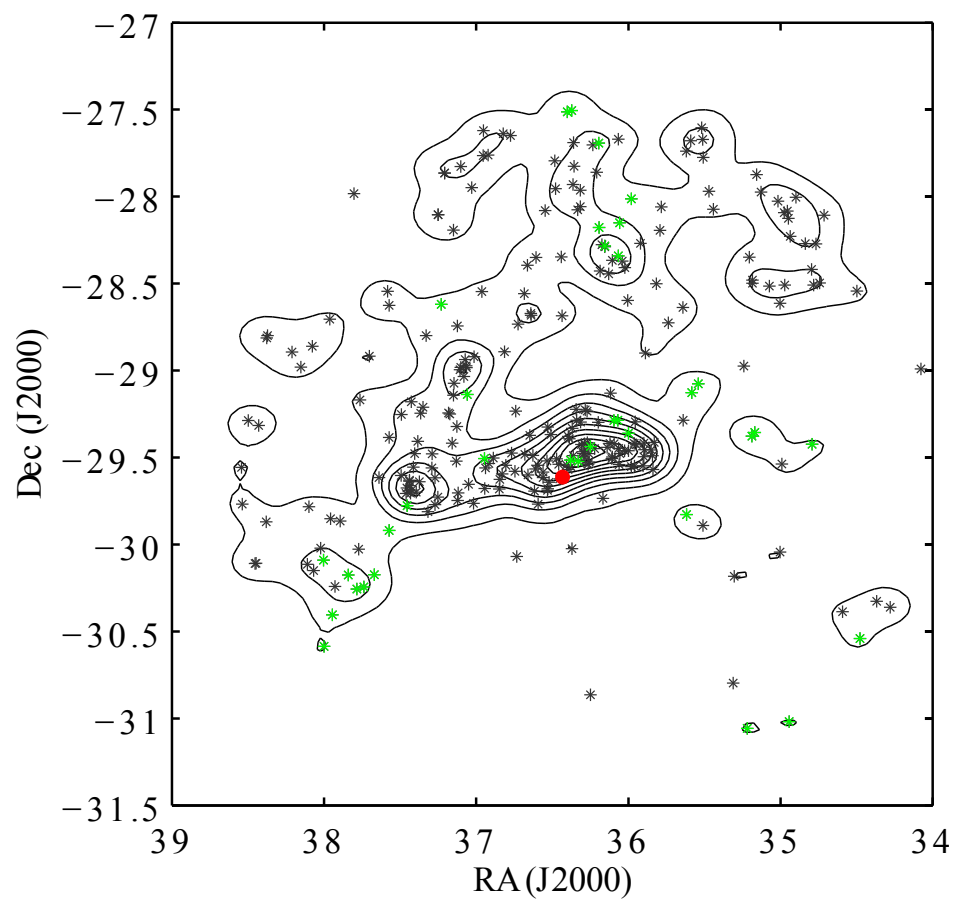

Figure 5.10: The distribution of galaxies with available redshifts in a two-degrees radius around RXCJ0225.1-2928. The grey stars show the galaxies with redshifts in range $0.055-0.063$ and green stars demonstrate the distribution of galaxies in range 0.063-0.068 which corresponds to the second peak in the histogram (Figure 5.9). The red dot shows the location of the cluster Abell S0258.

degrees radius around the cluster RXCJ0225.1-2928. The galaxy distribution around the cluster RXCJ0225.1-2928 shows a east-west elongation however, it is not possible to infer whether this elongation is only associated with the cluster RXCJ0225.1-2928 or the combination of the clusters RXCJ0225.1-2928 and A0S258.

\section{$\S$ 5.2.3. The Origin of Diffuse Emission in Cluster RXCJ0225.1-2928}

We have supplemented our radio analysis with the Murchison Widefield Array (MWA) commissioning image to determine the spectral index of the diffuse source with the aim of better classification of the radio structure. The radio source of interest has not been detected in the MWA image, thus, we have used the local $3 \times \sigma_{r m s}$ measurement as the upper limit flux. We matched the resolution of our ATCA low-resolution image to the resolution of the MWA commissioning image at $178 \mathrm{MHz}$ and we found that the undetected radio emission should have appeared as a point source in the MWA image. We have used a linear fit to the data in log-scale plot and the upper-limit of the integrated spectral index was estimated to be 0.48 for two data points (MWA and this work). In addition, since this source has remained undetected in the Very Large Array (VLA) Low-Frequency Sky Survey Redux (VLSSr). We have used the local $3 \times \sigma_{r m s}\left(\sigma_{r m s}=\right.$ $68 \mathrm{mJy}^{-1}$ beam $^{-1}$ ) measurement of the VLSSr image at $74 \mathrm{MHz}$ as the upper limit flux 
to derive the upper-limit of the $\alpha$ using three data points (VLSSr, MWA and this work). The derived upper-limit of the spectral index was measured as $0.59 \pm 1.43$. We note that the upper-limit spectral index does not preclude this as a real spectral index, it merely indicates that due to the shallow MWA and VLSSr images we cannot investigate the spectral index of the source properly.

The combination of our findings such as the location, lack of optical/IR counterpart, polarisation, morphology and the spectral index of the diffuse source suggested that this object has peculiar features and a robust classification of the source is not possible. In the following, we have discussed all the possibilities to address the origin of this diffuse radio source. The possible classifications are:

- a high redshift radio galaxy,

- a radio relic in RXCJ0225.1-2829,

- a dying radio galaxy, and

- a radio relic in a filament.

In the first case, the diffuse emission could be associated with a high redshift galaxy which has remained undetected in the DSS2 red image. Considering the DSS2 red image (UK Schmidt Telescope Red (IIIaF) second epoch) limiting magnitude of 21.5 (according to the SuperCOSMOS website) and using the r-band Hubble diagram for 3C galaxies ${ }^{1}$ (Snellen et al., 1996), the estimated minimum redshift of this object is $\approx 1.13$. According to the measured LAS of the diffuse radio source, the largest linear size at such high redshifts is $\geq 2 \mathrm{Mpc}$. The largest detected radio galaxy to date has the linear size of $4.69 \mathrm{Mpc}$ at redshift 0.3067 (Machalski et al., 2008). Thus, there is a possibility that this diffuse source is a radio galaxy at high redshift. However, the lack of a distinct core or hot spots in the high resolution image made from the visibilities with spatial frequencies greater than $18.75 \mathrm{k} \lambda$ (baselines longer than $3750 \mathrm{~m}$, antenna 6 only) is in disagreement with this scenario.

The detected diffuse source is located $\sim 1 \mathrm{Mpc}$ far from the centre of RXCJ0225.12928 (this distance is larger than reported radio relics cluster-centric distances in the literature) and has a measured LLS of $315 \mathrm{kpc}$ (at redshift of cluster). Since this cluster is an intermediate cluster according to X-ray data (Weißmann et al., 2013), we could assume that the diffuse radio source is a radio relic associated with the cluster RXCJ0225.1-2928. Diffuse radio sources with large cluster-centric distances have been previously reported in clusters Abell 2255 (Pizzo and de Bruyn, 2008) and Abell 2256 (van Weeren et al., 2009). The K-corrected power of the diffuse source was estimated

\footnotetext{
${ }^{1}$ The Third Cambridge Catalogue of Radio Sources (3C) is a radio source catalogue observed at 159 $\mathrm{MHz}$ and $178 \mathrm{MHz}$.
} 
to be $\mathrm{P}_{1.4}=3.33 \times 10^{23} \mathrm{~W} \mathrm{~Hz}^{-1}(\alpha=1.3$ is assumed) which lies at the lower end of the known radio relics to date. Given that this source is polarised and the distance to the cluster and its size are consistent with other radio relics in the literature, this object is likely to be a radio relic. According to Figure 16 presented by Feretti et al. (2012), the cluster-centric radius and the luminosity of this diffuse source seems in good agreement with other detected radio relics in the literature.

The other possibility is that this emission is associated with a relic of a dead radio galaxy. When the activity of the nuclei of a radio galaxy is halted, the radio source enters the last episode of its life-time known as "dying phase" and gradually disappears. It is believed that the fading period is an order of magnitude higher for the radio galaxies in the clusters with respect to the radio sources in the field (see Murgia et al., 2011 and references therein). Dying radio galaxies commonly do not have a radio core, jets and hot spots since the existence of such features is normally due to the continues activity of the nuclei. On the ground of observational evidence, the spectral index of the fading radio lobes is very steep $(\alpha \leq 1.5)$. Since our detected diffuse source in our ATCA observation has no core and hot spots, it can be a dying radio source candidate.

The final possibility is that this diffuse source is a radio relic in an inter-galactic filament. The optical analysis of the galaxies within a two degree radius around RXCJ0225.12929 indicated that there is a filament in the region of interest (see Figure 5.10). The accretion shocks in the filaments are known to be present due to accretion of the gas towards the potential well of the galaxy clusters. Since this source is polarised, this emission could be classified as a radio relic in a filament. A filamentary diffuse radio source has also been reported by Bagchi et al. (2002) and Giovannini et al. (2010). Brown and Rudnick (2009) also found a filamentary diffuse emission candidate, however, like our detection, they also have discussed the possibility of the source to be a dying radio galaxy.

In Table 5.4, a summary of the features of the detected diffuse emission and their validity with respect to the proposed possibilities of the emission origin are given. Due to current limited radio data available further speculation and conclusive classification could not be achieved.

These type of radio structures were previously presented in the literature; for instance, Slee et al. (2001) found four other diffuse radio sources in a galaxy cluster and the true classification of the sources remained elusive. Jamrozy et al. (2014) found a peculiar radio source in the cluster Abell 585 and they also considered various possibilities to classify the discovered radio structure, however, as with this case, a conclusion could not be achieved due to contradictory evidence. Likewise our detection in RXCJ0225.12928, Macario et al. (2014) detected peculiar diffuse radio objects around the cluster MACS J0520.7-1328 and due to insufficient evidence they could not accurately classify 
Table 5.4: Summary of the features of the detected diffuse emission and their validity with respect to the proposed possibilities of the emission origin.

\begin{tabular}{lcccc}
\hline \hline Emission feature & Cluster radio relic & Radio galaxy & Dying radio galaxy & Filamentary radio relic \\
\hline LLS & $\checkmark$ & $\checkmark$ & $\ldots$ & $\ldots$ \\
Polarisation & $\checkmark$ & $\checkmark$ & $\checkmark$ & $\checkmark$ \\
Lack of core & $\checkmark$ & $\times$ & $\checkmark$ & $\checkmark$ \\
Distant to the cluster & $\checkmark$ & $\checkmark$ & $\checkmark$ & $\ldots$ \\
Lack of optical ID & $\checkmark$ & $\times$ & $\times$ & $\checkmark$ \\
\hline
\end{tabular}

the radio sources.

We suggest that a follow-up radio observation with the CABB at $2.1 \mathrm{GHz}$ to provide an opportunity to accurately determine the spectral index of the source. The spectral index information is vital for better classification.

\section{$\S 5.3$. Summary and Conclusion}

In this chapter, we have presented the results of an investigation of low-resolution radio images of our observed REXCESS sub-sample. We have confirmed that there is a giant radio halo in the centre of the cluster A3888. The flux of the halo at $1.4 \mathrm{GHz}$ was measured as $27.57 \mathrm{mJy}$. The X-ray observation of A388 suggests that this cluster might be an intermediate cluster with some early stage of dynamical interactions in the centre. We performed the spectral index analysis of A3888 over the CABB band at $1.4,1.8,2.1$ and $2.4 \mathrm{GHz}$ and we measured the spectral index of the halo to be $\alpha=$ $1.48 \pm 0.14$. It is worthwhile to perform low frequency observations of this cluster with sufficient resolution (MWA II) to improve the accuracy of the integrated spectral index measurement.

We also have presented another diffuse object detected in cluster RXCJ0225.1-2928. Unfortunately, due to lack of sufficient resolution, internal structure of this radio emission is not clear. In addition, an accurate spectral index map and more precise integrated spectral index are vital to distinguish between different possibilities to address the origin of the emission. Thus, new radio observations in CABB mode particularly in any 6 $\mathrm{km}$ array configuration is strongly suggested.

It is highly likely that more of this kind of diffuse sources await discovery with more sensitive and higher-resolution radio observations. Future radio surveys such as Evolutionary Map of the Universe (EMU; Norris et al., 2011) with its unprecedent sensitivity is expected to detect many of such peculiar emissions. Additionally, all sky infra-red (IR) surveys with the James Webb Space Telescope (JWST; Gardner, 2015) in the future are expected to provide a very deep map of the Universe up to the intermediate redshifts. These observations could discover many undetected IR counterparts of radio 
galaxies. From an optical perspective, future optical telescopes such as the European Extremely Large Telescope (EELT; see Basden et al., 2014 and references therein) and Giant Magellan Telescope (GMT) are expected to cover more of Southern sky and push the limits of current optical observations. Hence, many of the undetected optical IDs of radio galaxies are expected to be discovered in future. 


\section{§6. Optical Analysis of Abell 3888}

\section{$\S 6.1$. Introduction}

In the last few decades, with the advent of multi-object spectrograph, astronomers have been capable of investigating the redshift information of galaxies over large areas of the sky. Such wide-field analysis of spectroscopic surveys are thus important tools to unravel the history and the underlying physics that plays a major role in the large scale structure formation in our Universe. Recent large redshift survey campaigns e.g. the Six-degree-Field Galaxy Survey (6dFGS, Jones et al., 2009), the Two-degree-Field Galaxy Redshift Survey (2dFGRS, Colless, 1999) and the Sloan Digital Sky Survey (SDSS, Abazajian et al., 2009) have revealed that the visible matter creates web-like filamentary structures of the putative "cosmic web" on scales larger than a few megaparsecs. There are also megaparsec scale voids which are regions that harbour only small numbers of galaxies and fill the space between the filaments.

In conjunction with the large redshift surveys mentioned above, large N-body simulations such as the Millennium Run Observatory (MRObs) project (Overzier et al., 2013) are used to understand the underlying physics of the structure formation in the Universe. The N-body simulations have confirmed the findings of the large redshift surveys; galaxies in the Universe form in large scales features known as filaments, sheets, superclusters and cluster of galaxies. It is known that the interconnecting nodes of filaments are the most common places to host galaxy clusters.

One of the remarkable results from the aforementioned redshift censuses is the confirmation of current "hierarchical model of cosmology" (Peebles, 1980) which is currently largely accepted as the best model to explain large structure formation in the Universe. In this model, smaller units e.g. galaxies and galaxy groups come together over a long period of time and merge to form larger units such as galaxy clusters.

According to this model of cosmology, merging of galaxy clusters is a very common phenomena and might happen multiple times during the life-time of galaxy clusters. It is believed that cluster merging is a key parameter in formation and evolution of galaxy clusters. Moreover, merging has a significant impact on cluster properties such as velocity dispersion, temperature and mass. As explained in $\S 2.1$, in addition, the hot, X-ray emitting gas which fills a vast region in the central part of the clusters (also known as Intra-Cluster Medium, ICM), is affected dramatically in the merging process via the powerful shocks and turbulence resulting from the cluster merger.

Merging of clusters often generates clumps of galaxies within the cluster volume 
and this change in galaxy volumetric density is known as "substructure". Detection of such structure is an important tool to infer the cluster's dynamics and morphology. The presence of substructure indicates that the cluster is dynamically young and might be a signpost of an ongoing or past merger event.

The dynamical status of a cluster is determined via the optical spectroscopy of member galaxies and X-ray observations of the ICM. The brightness distribution of the X-ray images in merging clusters are asymmetric and often exhibit substructures. However, it should be noted that the X-ray observations of a cluster are merely useful to detect merging events in or close to the plane of sky and are not sufficiently sensitive to the merging activities occur along our line-of-sight (LOS) (Johnston-Hollitt et al., 2008; Owers et al., 2009). On the other hand, optical observations of clusters carry information about the merging not only happening in our LOS but in most of the cases with various merging axes. Thus, the combination of the optical and X-ray substructure analysis of clusters is the most robust method to detect merging and its primary axis in clusters.

In this chapter, we present the results of substructure analysis of the galaxy cluster Abell 3888 (A3888). This cluster has been previously studied at different wavelengths in large surveys such as the Representative XMM-Newton Cluster Structure Survey (REXCESS, Böhringer et al., 2007), the Las Campanas AAT Rich Cluster Survey (LARCS, Pimbblet et al., 2006), the Local Cluster Substructure Survey (LoCuSS, Plagge et al., 2010) and is presented in the Planck early results VIII (Planck Collaboration et al., 2011).

In $\S 5.1$, we have presented the information and properties of the discovered giant radio halo in A3888. We have also explained all the previously known optical properties and the X-ray substructure analysis results of this cluster which have been presented in multiple publications.

With the aim of studying the dynamics of the cluster from an optical perspective, we searched the NASA Extragalactic Database (NED) in a one degree radius around A3888 for available redshifts. There were 788 reliable spectroscopic redshifts available in the literature primarily from the LARCS project (Pimbblet et al., 2006). The redshift of the cluster is reported to be $0.1528 \pm 0.0003$ based on 201 candidate member galaxies up to $16 \mathrm{Mpc}$ away from the cluster centre (Pimbblet et al., 2006). Despite the 201 possible members, there were only 72 galaxies with reliable redshifts in a $1 \mathrm{Mpc}$ radius around the cluster. For multiple reasons such as: 1) the importance of investigating the dynamical status of halo clusters to distinguish between the halo generation models, 2) contradictory information about the X-ray substructure analysis of $A 3888,3$ ) the lack of detailed optical substructure analysis in the literature and 4) the small number of available redshifts in the core of A3888, we opted for new optical observations of A3888 with the AAOmega spectrograph and performed an optical analysis with the 
aim of achieving clear insight into the morphology of the cluster. In the following we assume the $\Lambda \mathrm{CDM}$ cosmology with $\mathrm{H}_{0}=67 \mathrm{~km} \mathrm{~s}^{-1} \mathrm{Mpc}^{-1}, \Omega_{m}=0.32$ and $\Omega_{\Lambda}=0.68$ (Planck Collaboration et al., 2013).

\section{§6.2. AAOmega Observation of A3888}

We examined the Digitised Sky Survey (DSS) blue image and found that roughly two thirds of the galaxies up to the cluster's Abell radius $\left(\frac{1.72^{\prime}}{z_{c l}}=12^{\prime}\right)$ did not have measured redshifts and the aforementioned redshift sample was not isotropic in terms of coverage. Careful examination of the SuperCOSMOS catalogue, which has an astrometric accuracy of $0.3^{\prime \prime}$ and completeness of $90 \%$ up to $\mathrm{m}_{b}=21$ (Hambly et al., 2001b), suggested that the majority of the galaxies without measured redshift fall between blue magnitudes of 18 and 21.5.

AAOmega is a fibre-fed spectrograph which provides multi-object spectroscopy and is mounted at the Anglo Australian Telescope (AAT). The spectrograph has 400 fibres covering a two-degree field when projected on sky making it an ideal instrument for examining the optical substructure in nearby southern clusters. Each fibre has a $2^{\prime \prime}$ diameter when it is projected on the sky and all fibres are placed by a robot. The accuracy of locating the fibre on the target is about $0.3^{\prime \prime}$ on the projected sky which is well matched to the SuperCOSMOS astrometry. During observations, eight guide star fibres are used for accurate telescope positioning. AAOmega has a dual beam which allows the observations of a wide range of wavelengths.

Since the number of targets in the AAOmega spectrograph configuration input file should not exceed 800, we first selected all the galaxies with $17.5 \leq m_{b} \leq 21.5$ and then we sorted the galaxies based on their cluster-centric radius; as a result, there were 790 galaxies up to $30^{\prime}$ radius from the centre of the cluster. All of the 790 galaxies were selected as our science targets.

Although there are only 400 optical fibres available on the AAOmega spectrograph, for the sake of homogeneous observations it is recommended to include between 500 and 800 science targets in the configuration file. This is particularly important when the centre of the field is over-dense as the physical size of the fibre buttons prevents very close galaxies from simultaneously being observed. This implies one should have a larger number of targets and optimise accordingly using the field configuration software produced by the observatory. Figure 6.1 shows the configuration of all 400 fibres for our AAOmega observation.

Our new observations of the cluster A3888 were taken on the 9th and 10th of May 2013 with the AAT in service mode. The total integration time of three hours was observed in half an hour blocks so as to reduce cosmic ray contamination. The typical 


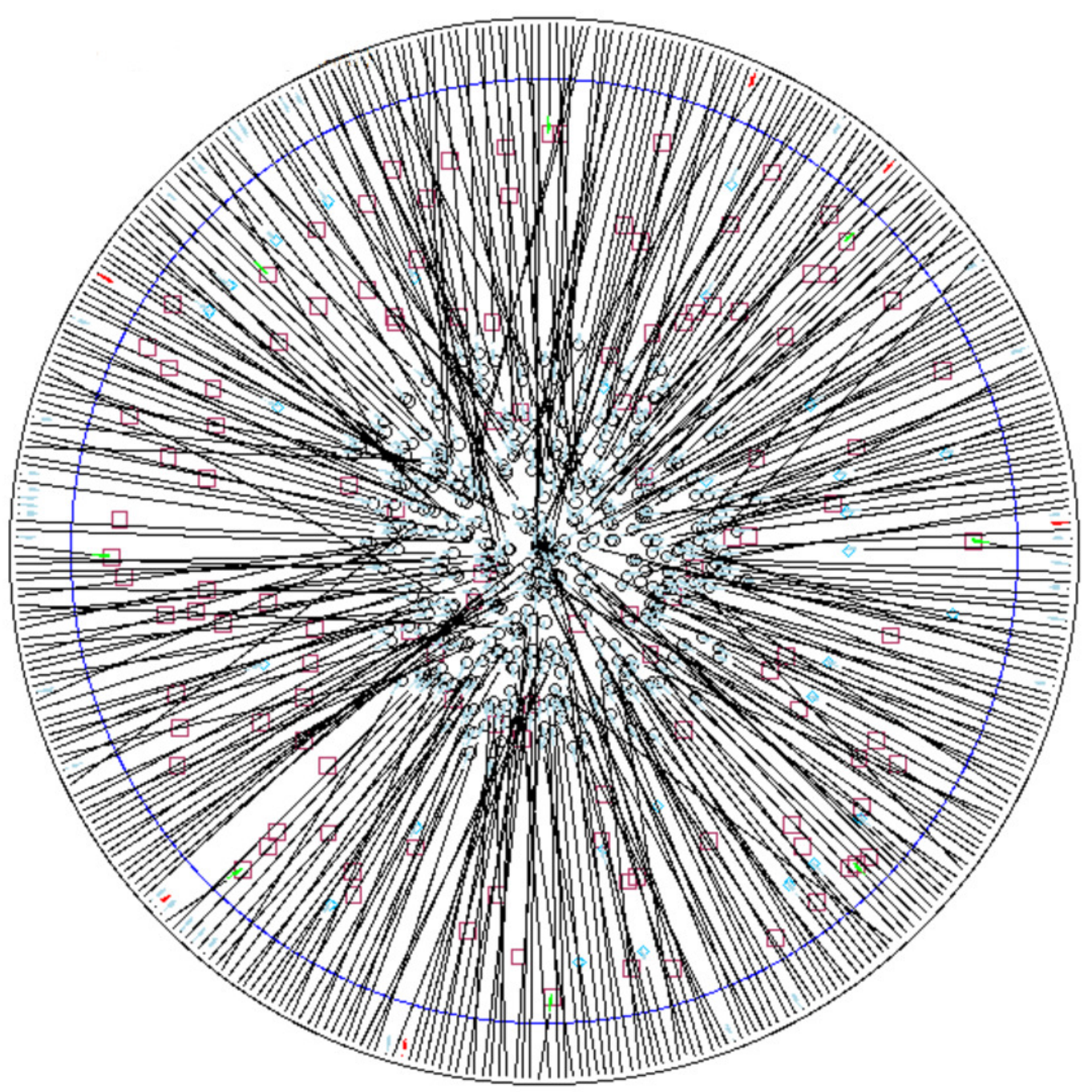

Figure 6.1: The optical fibre configuration over the two-degree field. A total of 400 fibres (solid black lines) are available to be allocated to sky positions (red squares), guide stars (blue diamonds) and science targets (black circles). The image shows the configuration used for the A3888 observations.

seeing was $1.5^{\prime \prime}$ to $2^{\prime \prime}$ for both nights. A total of 337 optical fibres were allocated to the science targets. There were 8 and 25 fibres allocated to guide stars and sky positions, respectively and 30 fibres remained un-allocated since some of them were broken or they had been detached during the fibre un-crossing procedure. All the sky positions were visually inspected using the DSS blue image to ensure that there were no optical sources within a $5^{\prime \prime}$ radius around each of the sky positions. We used a low to medium spectral resolution where the blue arm has a $580 \mathrm{~V}$ grating at the central wavelength of $4800 \AA$ and the red arm has a $385 \mathrm{R}$ grating with the central wavelength $7250 \AA$. This set of gratings provide a spectral resolutions of $3.5 \AA$ and $5.3 \AA$ at $4800 \AA$ and $7250 \AA$, respectively. The combined wavelength coverage resultant from both arms was 3700 $8800 \AA$. This wavelength coverage allows detection of emission lines such as $\mathrm{H} \alpha, \mathrm{H} \beta$, $\mathrm{H} \delta, \mathrm{H} \gamma, \mathrm{NII}, \mathrm{SII},[\mathrm{OI}],[\mathrm{OII}]$ and [OIII], and absorption lines such as $\mathrm{H}, \mathrm{K}, \mathrm{G}, \mathrm{Na}$ I and 
$\mathrm{Mg}$ up to redshift of $\sim 0.5$ and it is well matched to the redshifts of nearby clusters.

\section{§6.3. Data Reduction and Redshift Determination}

The observatory staff performed the observations collecting the required calibration files including bias, dark, arc and flat field frames. The data were automatically reduced using the AAO data reduction pipeline software "2dfdr" (Colless et al., 2001). We briefly outline the calibration procedure here. First, the bias image is generated during the reading process of the CCD whilst it is not exposed to light over a zero second interval to measure the inherent noise generated by the CCD amplifiers. Next, a dark frame is taken when the CCD is not exposed to light (shutter closed) but the exposure time is identical to the exposure time of the object frames (science image). Dark frames are usually used to measure the thermal noise across the CCD array. Flat field exposures are then taken and used to correct the photon capture variation of the pixels across the CCD. Flat fields are used to illuminate a CCD in a long exposure to generate a calibration image with a very high signal-to-noise ratio. Finally, $\mathrm{CuAr}$, helium and $\mathrm{FeAr}$ arc lamp images are usually used to generate spectral lines to calibrate the wavelength of the object frames.

In addition to above instrumental calibration issues, the sky itself also needs to be accounted for. Cosmic rays hit the CCDs during an exposure producing bright patches which are accounted for with a sigma-clipping algorithm within 2 dfdr. Additionally, water vapour, $\mathrm{O}_{3}$ and $\mathrm{O}_{2}$ in the Earth's atmosphere can cause significant imprints in the target's spectra by generating false absorption features which are usually referred to as Telluric absorption spectral lines. These absorption lines are dominant in near the Infra-red (IR) and visible regions of the spectra. There are also contaminating emission lines known as sky lines that are mainly caused by $\mathrm{NaI}$ and $\mathrm{OH}$ in the atmosphere. The 2dfdr software calibrates and subtracts night sky lines (atmospheric emission and absorption lines) automatically for each multi-object frame and for each arm separately. After reducing each frame, all of the frames from each spectrograph were combined. Afterwards, the coadded frames for the blue and red arms are spliced together.

This final reduced and spliced image can be used as an input file to any spectroscopic redshift fitting software. In this work, we used the "RUNZ" code to extract the redshifts from our spectra (see Appendix D.1 for the full dataset). RUNZ was originally developed by Dr Will Sutherland with the aim of using it in the two degree field Galaxy Redshift Survey (2dfGRS, Colless et al., 2001). Currently, RUNZ is available and maintained by A/Prof. Scott Croom who kindly provided the software to us.

RUNZ measures two redshifts simultaneously employing two different methods:

- Emission line redshift: An individual redshift is measured by fitting a Gaussian 
to each emission feature in the spectrum and the final redshift is assigned to an object by calculating the mean value of the variance weighted measured redshifts.

- Cross-correlation redshift: This redshift is assigned to each spectrum by employing the standard cross-correlation technique (Tonry and Davis, 1979). Here, each spectrum is cross-correlated to a set of stellar and galaxy spectral templates. The redshift with the highest peak of the correlation coefficient is considered as the final cross-correlated redshift. The technique is primarily concerned with absorption features, and the success of this method depends strongly on the quality of the input templates.

The Sloan Digital Sky Survey data release 7 (SDSS DR7) galactic and stellar spectroscopic templates were used in order to determine the cross-correlated redshifts. In addition to the estimated redshift, RUNZ also returns an automatically assigned redshift quality flag "q" ( ranging from 1-5) to describe the reliability of the estimated redshift. Redshifts with $\mathrm{q} \leq 2$ are considered unreliable, $\mathrm{q}=3$ is considered likely and redshifts with $\mathrm{q} \geq 4$ are very reliable. For the qualitative definitions of the automatic quality flags we refer the reader to (Colless et al., 2001). All of the spectra were visually inspected to confirm the accuracy of the redshift measurement and the software-determined redshift quality flag. The measured redshift (emission or absorption) with the higher quality flag was reported as the final redshift for each spectrum. There was no spectrum with the same quality flags for both the emission and the absorption measured redshifts.

In some cases for absorption redshifts, we assigned a different redshift quality from the one which was assigned by the RUNZ automatically. We manually assigned a quality of 4 to redshifts where both $\mathrm{H}$ and $\mathrm{K}$ absorption lines were present. In the case that $\mathrm{H}$ and $\mathrm{K}$ and at least one of the other absorption line $(\mathrm{Mg}, \mathrm{Na}, \mathrm{G}, \mathrm{H} \delta$ ) were present, a quality of 5 was assigned to the redshift. If merely $\mathrm{H}$ or $\mathrm{K}$ and at least two of the other absorption lines were present a quality of 4 was assigned. In the case that neither $\mathrm{H}$ or $\mathrm{K}$ were present if all the other absorption lines were present then the quality of 3 was given. In the case that only one or two absorption lines $(\mathrm{Mg}, \mathrm{Na}, \mathrm{G}, \mathrm{H} \delta$ ) were present then a quality of 2 was given to the redshift. In the case that no absorption features were detectable then a quality of 1 was assigned. In Figure 6.2, examples of emission and absorption spectra and associated fitted lines from RUNZ are shown.

\section{§6.4. Redshift Extraction Results}

As mentioned earlier, 337 of our science targets were observed with AAOmega. Following the redshift extraction using RUNZ, we found that 41 objects were stars which signifies the uncertainty level of the SuperCOSMOS star/galaxy classification. Hambly et al. (2001a) estimated the classification to be $\sim 90 \%$ accurate down to $\mathrm{m}_{b}=21$ which 


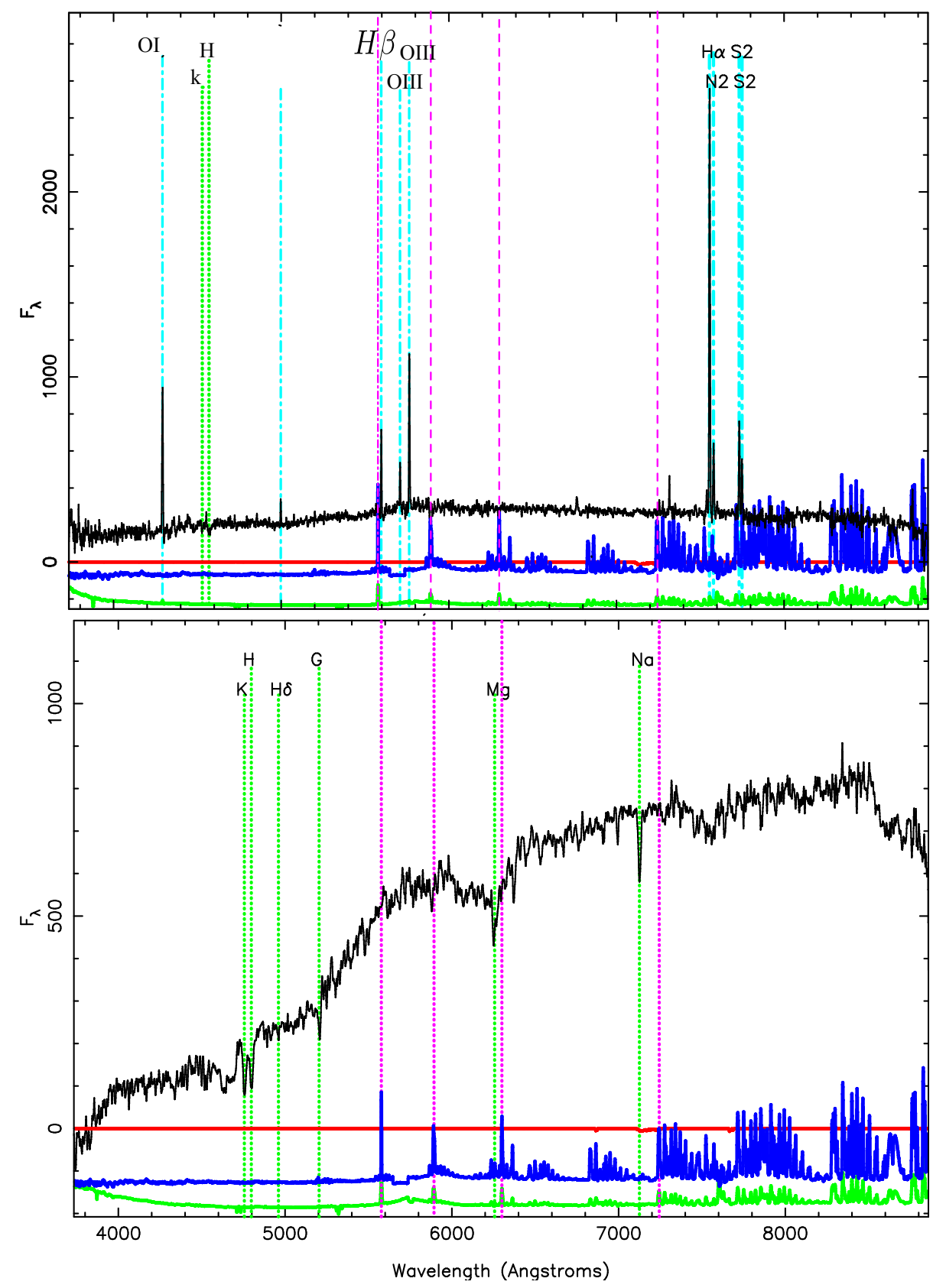

Figure 6.2: Two examples of reduced spectra are shown. Top panel: An absorption line galaxy in the field with redshift quality 5. Bottom panel: An emission line galaxy in the field with redshift quality of 5. In each plot, the galaxy spectrum (black line), residual spectrum after sky subtraction (green line), sky spectrum (yellow line) and the Telluric absorption spectrum (red line) are superimposed. The emission (dotted cyan lines) and absorption (dotted green lines) features at the best fitted redshift are also shown. The strongest night sky emission lines are demonstrated with dotted pink lines.

is consistent with our finding ( $88 \%$ ). A total of 42 measured redshifts from objects with fairly faint blue magnitudes $\left(18.7<m_{b}<20.5\right)$ were assigned $\mathrm{q} \leq 2$ thus they were not included in our analysis. Colless et al. (2001) pointed out that the successful redshift determination rate decreases significantly beyond $m_{b}=19$ and our detection success rate $(87.5 \%)$ is in agreement with their finding for the entire $2 \mathrm{dF}$ galaxy redshift survey. Af- 


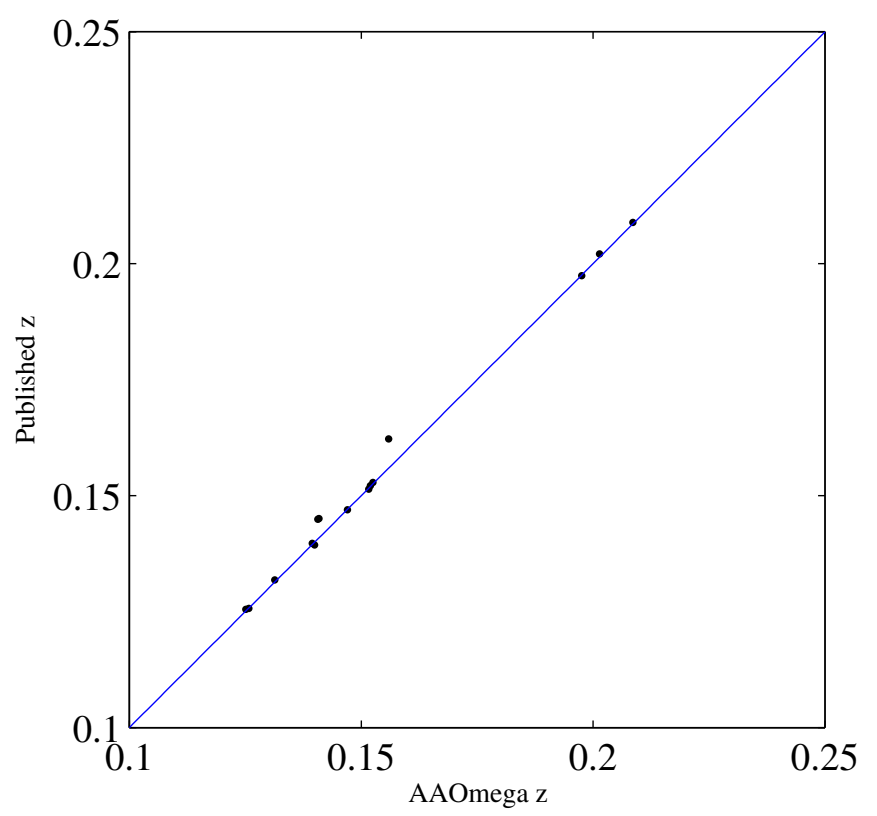

Figure 6.3: Common objects between the AAOmega observations and the literature, the error bars are not shown due to being smaller than the size of the dots on the plot. The solid blue line is the one-to-one relation.

ter the removal of the misclassified stars and non-detected galaxies the remaining 254 targets observed here were classified as galaxies with reliable redshifts.

We have supplemented our redshift sample with available redshifts in the literature. We searched the NED up to one degree radius from the core of A3888 for reliable spectroscopic redshifts. We then co-added our spectroscopic redshifts to the published redshifts to build a final redshift sample containing 1027 spectroscopic redshifts. Assuming a search radius of $3^{\prime \prime}$ we found a total of 15 galaxies in common between our AAOmega observation and the redshifts from the literature. For cases where an object had two redshift measurements, our redshift was chosen if $q \geq 4$ otherwise the redshift from the literature was used in our analysis. For all such galaxies, the DSS blue image was visually inspected to ensure that the cross-matching process did not conflate two separate objects and they were indeed true cross-matches. Figure 6.3 shows the consistency between our extracted redshifts and the redshifts from the literature (for our 15 common redshifts). The plot shows that the majority of our common redshifts are in good agreement with the published redshifts.

A colour-coded histogram of the combined redshifts from the final catalogue in a one degree radius around A3888 is shown in Figure 6.4. The redshift distribution clearly shows the existence of seven distinct populations around A3888. The structures span the redshift range $0.02-0.31$. The redshift range associated with each population was determined based on conspicuous gaps in the redshift histogram. In Table 6.1, the redshift range and the number of galaxies in each of identified velocity groups in the redshift 
Table 6.1: Details of the velocity groups and number of galaxies in each redshift slice in a one degree radius around A3888. The largest structure in velocity is clearly A3888 and its outskirts with 469 member galaxies and an extent of $\sim 10 \mathrm{Mpc}$ in radius.

\begin{tabular}{ll}
\hline \hline Redshift Range & $\mathrm{N}_{\text {gal }}$ \\
\hline $0.0284-0.0475$ & 80 \\
$0.0530-0.0875$ & 163 \\
$0.0880-0.1190$ & 67 \\
$0.1200-0.1850$ & 469 \\
$0.1850-0.2220$ & 156 \\
$0.2230-0.2650$ & 43 \\
$0.2670-0.3080$ & 28 \\
\hline
\end{tabular}

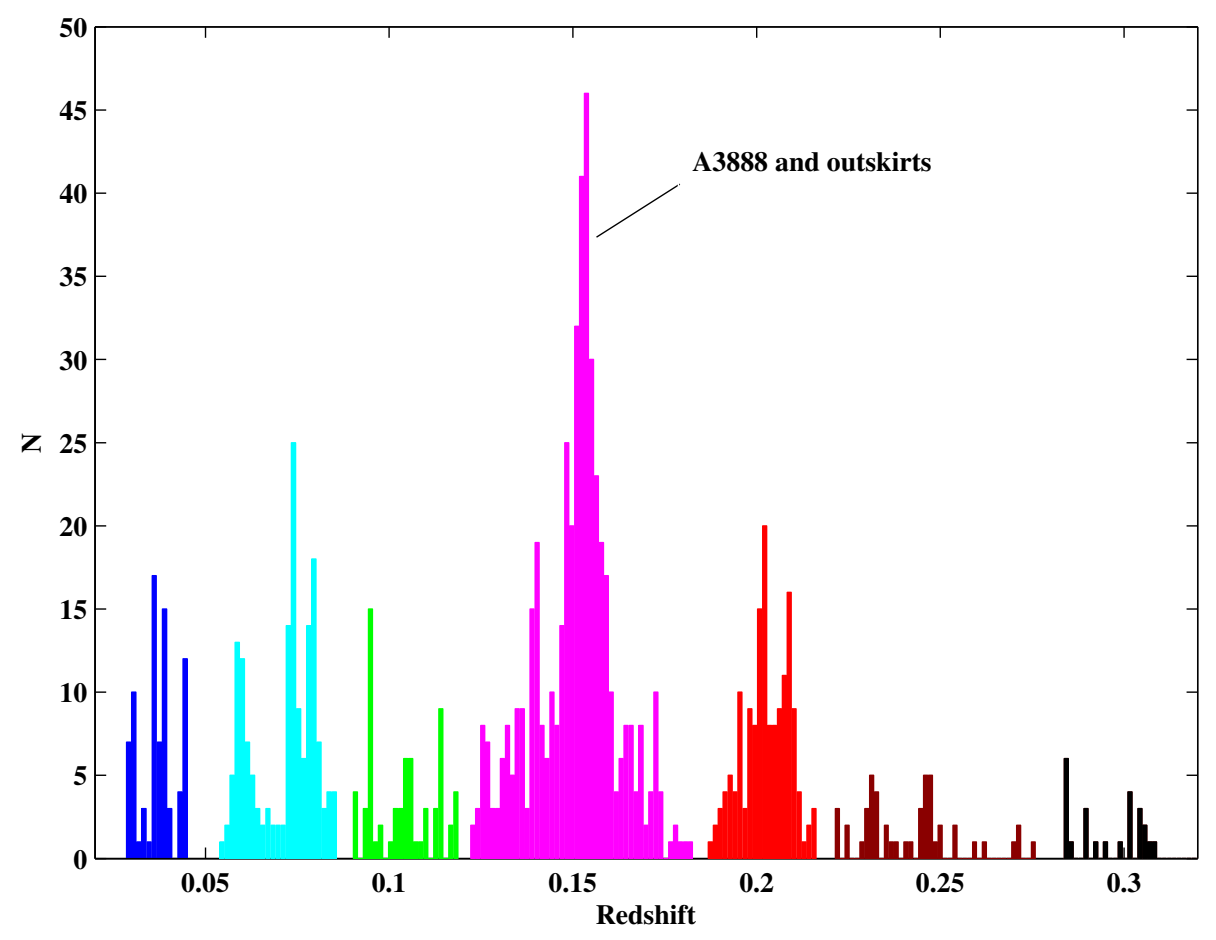

Figure 6.4: The colour-coded redshift histogram of the combined redshifts (AAOmega+literature) in one degree radius around the core of A3888. According to this plot, there are seven distinguishable structures up to the redshift of 0.35 . See Table 6.1 for details.

sample are shown. There were an extra eight redshifts scattered in the redshift slice from 0.0001 to 0.0108 and 13 in redshift slice from $0.310-0.458$ which are not included in Table 6.1 and in Figure 6.4. 

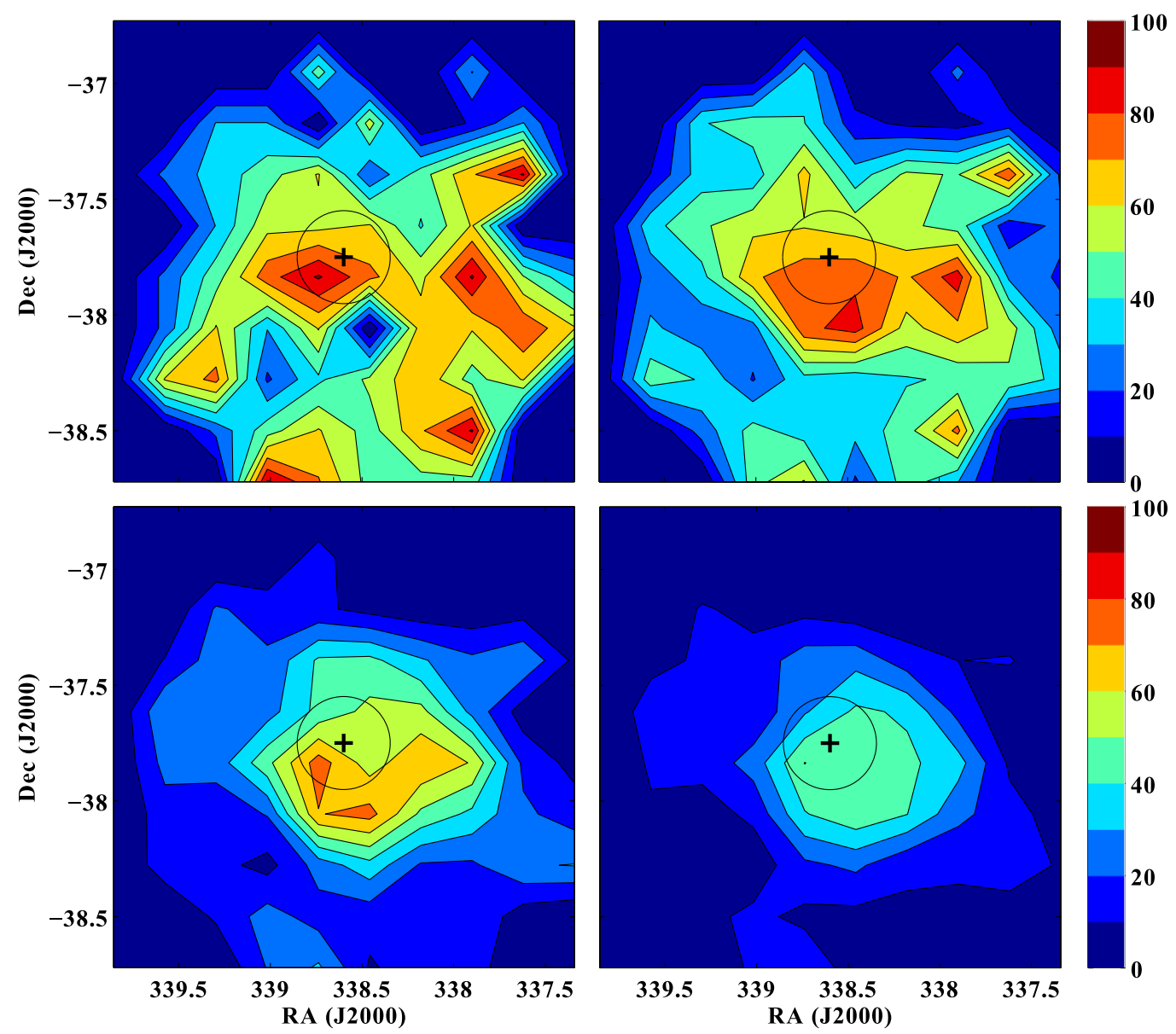

Figure 6.5: Spectroscopic completeness map of A3888. Top left panel: spectroscopic completeness in $m_{b} \leq 18$. Top right panel: completeness in $m_{b} \leq 19$. Bottom left panel: completeness in $m_{b} \leq 20$. Bottom right panel: completeness in $m_{b} \leq 21$. Black cross shows the position of A3888 and the black circle shows a region with radius of $2 \mathrm{Mpc}$ around A3888.

\section{§6.5. Spectroscopic Redshift Completeness}

Completeness of our redshift sample was determined by calculating the ratio of galaxy number density in the combined redshift catalogue (AAOmega+literature with the upper limit of $\mathrm{m}_{b}=21.32$ ) to the number density of galaxies with available blue magnitudes in the SuperCOSMOS catalogue which is $90 \%$ complete up to blue magnitude of 21 . There were 937 galaxies out of 1027 galaxies in our redshift sample which had available blue magnitude in the SuperCOSMOS catalogue.

The final spectroscopic completeness was scaled by multiplying the ratio of the number of redshifts with available blue magnitude to the total number of available redshifts. Figure 6.5 shows the cumulative spectroscopic completeness of our final redshift sample as a function of blue magnitude. As it is expected, the spectroscopic completeness decreases whilst the blue magnitude cut increases from top left to bottom right. In some regions the completeness increases, however this unexpected behaviour has been 

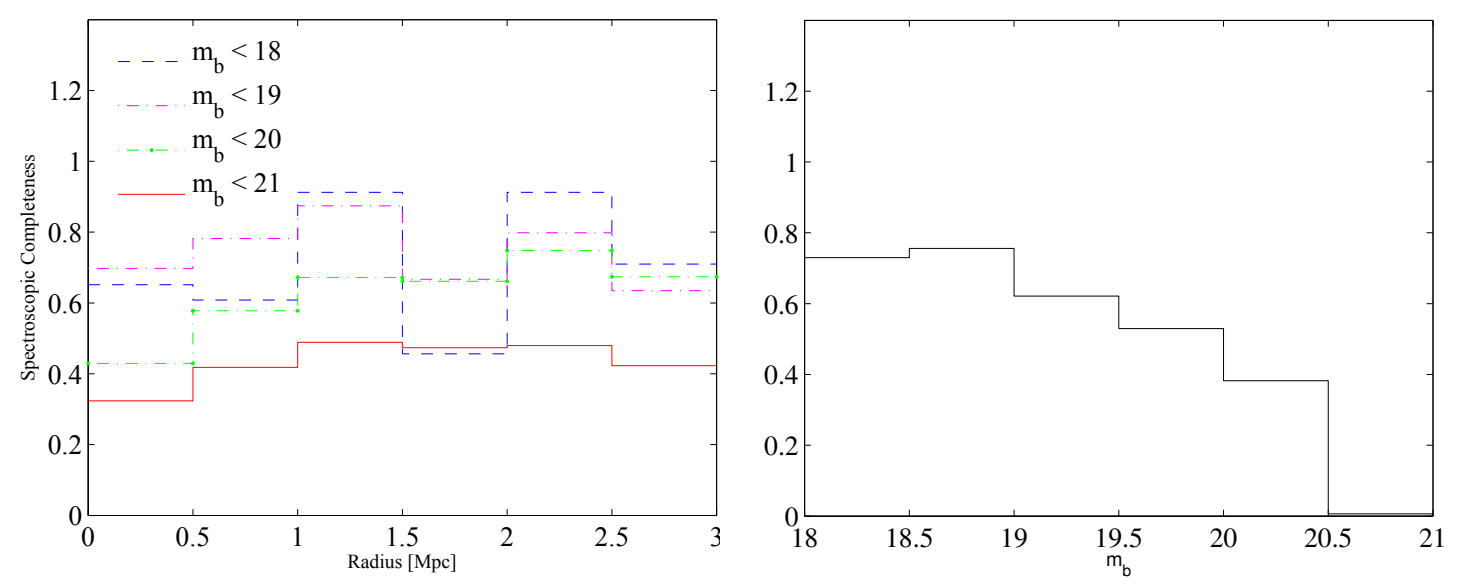

Figure 6.6: Left panel: cumulative spectroscopic redshift completeness in radial bins of $500 \mathrm{kpc}$. Right panel: spectroscopic redshift completeness as a function of magnitude within $3 \mathrm{Mpc}$ radius from the cluster core.

previously seen in other works. For instance, Owers et al. (2011) presented the completeness of the spectroscopic observation of Abell 2744 with the AAOmega and they have observed the same behaviour in some regions. This is suggestive of an anisotropic redshift coverage as a function of magnitude. Figure 6.5 also shows that the completeness decreases whilst moving radially outwards which is in agreement with giving a low priority to the outermost regions which are considered as the outskirts of A3888 in our AAOmega configuration procedure. In Figure 6.6 the left plot shows the cumulative spectroscopic completeness as a function of radius from the core of A3888. According to this plot the completeness is above $30 \%$ in all radial bins for all magnitude ranges. The low completeness value in the first radial bin of $500 \mathrm{kpc}$ from the cluster core indicates that the central region of the cluster was extremely over-dense that the spectroscopy with the AAOmega was unable to cover the very dense cluster core due to physical size of the fibre buttons and the least required fibre separation of $30^{\prime \prime}$. The right plot in Figure 6.6 shows the redshift completeness in different magnitude bins within 3 $\mathrm{Mpc}$ radius from A3888. This plot shows that the spectroscopic completeness is at least $38 \%$ within a $3 \mathrm{Mpc}$ radius from A3888 core in all magnitude bins whilst the magnitude is brighter than $\mathrm{m}_{b}=20.5$.

\section{$\S 6.6$. Cluster Membership}

Decades of optical observations of galaxy clusters have revealed that the majority of galaxy clusters host a single or multiple substructures allowing a broad classification as either dynamic or relaxed systems, respectively. As mentioned earlier, the presence of substructure in a cluster is likely indicative of ongoing dynamical interactions. Substruc- 
tures may be formed throughout the infall of individual galaxies or galaxy groups into a relaxed cluster or during the merging of two or more entire galaxy clusters. According to theoretical studies and simulations of galaxy clusters, member galaxies of virialised clusters are expected to exhibit a well-behaved Gaussian velocity distribution (Owers et al., 2009). Therefore, any sign of non-Gaussian behaviour in the velocity distribution can be a signpost of certain conditions such as existence of fore/background interloper galaxies and more importantly, substructure in the cluster (Pinkney et al., 1996; Owers et al., 2009). However, it should be noted that a well established single Gaussian in velocity distribution does not necessarily indicate that the cluster is in dynamical equilibrium (Owers et al., 2009). For instance, the cluster Abell 3667, which has been well studied in the past, is known to be a highly disturbed cluster, however Johnston-Hollitt et al. (2008) and Owers et al. (2009) pointed out that this cluster has a well described Gaussian velocity distribution as measured along the line of sight, transverse to the known plane of the sky merger axis.

Additionally, interlopers may cause non-Gaussian behaviour in the velocity distribution which can be mistaken as true substructure (Owers et al., 2009). Hence, it is imperative to minimise fore/background galaxy contamination in the cluster field. To achieve this, spatial and LOS peculiar velocity distributions of galaxies are used solely or in a combined manner to determine the cluster members before any further substructure analysis is under taken.

The interloper rejection and cluster member selection for A3888 was performed combining two methods: a Density-Based Spatial Clustering of Applications with Noise (DBSCAN) algorithm (Ester et al., 1996) and the caustic technique (Diaferio and Geller, 1997).

\section{$\S$ 6.6.1. DBSCAN Clustering Algorithm}

As described earlier in $\S 6.4$, the initial cut of the foreground and background galaxies was carried out by identifying the velocity gaps in the redshift histogram. Further outlier removal was performed by employing DBSCAN method. This technique has been recently used in different fields of Astrophysics to statistically aggregate data in complex, irregularly sampled datasets, for instance Tramacere and Vecchio (2013) and Carlson et al. (2013) adopted it in analysing the Fermi-LAT gamma ray datasets, and Dehghan and Johnston-Hollitt (2014) used this method to detect the galaxy groups and clusters in the Chandra Deep Field-South (CDFS). We used the DBSCAN function of the open source package fpc ("fixed point clusters") implemented in the Comprehensive R Archive Network (CRAN). The DBSCAN function in this package follows the procedure introduced by Ester et al. (1996).

The DBSCAN technique requires two user-defined input parameters: the minimum 
number of points (MinPts) which defines the minimum accepted number of the structure members, and the neighbourhood radius to search for the members associated with each structure (Ester et al., 1996). All of the data points have a classification as core, border or noise. This method is mainly based on calculating the density of individual data points in the sample. The number of all data points within the radius "Eps" is referred to as the density of that particular point. In the calculation of the density, the data point, itself, should be taken into account (Ester et al., 1996). In the case that the number density of a point is greater than MinPts, that point is considered as a core point. A border point is not a core point but it is still within the Eps radius vicinity of a core point and has lower number density value. The points which are not in the Eps radius proximity of the core points are classified as noise. All the steps in the DBSCAN procedure are described below:

1. An arbitrary data point is chosen as a starting point. If the density of that point exceeds the MinPts value, then that point is considered as a core point, otherwise another non-classified data point will be retrieved and the same procedure is repeated. Once all the core points are found, the process finds the border and noise points until all the data points are classified. Then, all the noise points will be removed from the data points and will not be further involved in processing.

2. An arbitrary core point is chosen and is allocated to the first structure. Then, any core point in the Eps-neighbourhood of that core point is also allocated to the first structure. This process recursively searches for other non-allocated core points within the Eps radius around each core point and allocates them all to the first structure. This procedure is repeated till all the border points in the Epsneighbourhood of allocated core points are also allocated to the first structure. The process is halted when there are no core or border point left to be assigned to the structure.

3. A new core point which is not a member of any pre-detected cluster is retrieved and step 2 is repeated and further clusters are discovered.

4. All the steps are repeated until all the data points are assigned to a cluster.

The DBSCAN method has some advantages over the widely used Gaussian mixture model clustering algorithm in which velocity groupings are identified by determining a combination of various fitted Gaussians to the dataset (Ashman et al., 1994). The Gaussian mixture clustering approach requires an initial guess of the total number of groupings and the mean of each fitted Gaussian distribution. However, DBSCAN does not need a prior guess of the number of structures. In addition, DBSCAN is not restricted to detect any particular symmetric shape (circle, oval, ...) for the groupings. 


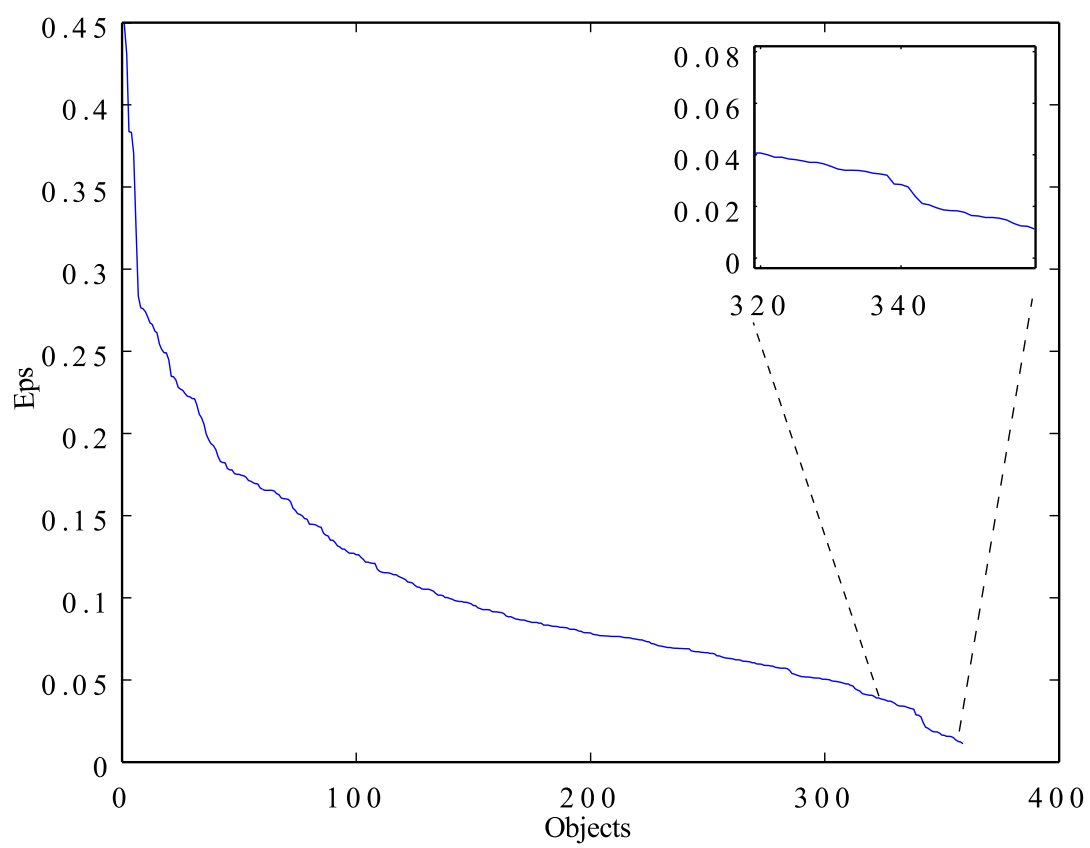

Figure 6.7: The sorted k-dist plot with $\mathrm{k}=6$. The zoomed inset shows the change in the slope of this k-dist graph. The values which are corresponding to strong changes in the slope are used as the Eps values for the DBSCAN algorithm. The vertical axis has units of degrees and the horizontal axis has no physical unit. The 6-dist sorted plot shows an abrupt change around Eps $=0.027$ ( $\sim 270 \mathrm{kpc}$ at redshift of cluster) and accordingly, the detected structures had proper scales.

However, the initial estimate of input parameters (MinPts, Eps) can broadly affect the detected structures by DBSCAN. To avoid blind guessing of the input parameters, Ester et al. (1996) suggested to use a so called k-dist plot to choose the Eps value.

The k-dist plot can be constructed following two steps: 1) the distance to the $\mathrm{k}^{\text {th }}$ ( $\mathrm{k}=$ MinPts-1) nearest neighbour for each data point is calculated, 2) the distance values should be sorted in descending manner and plotted. Since the k value is MinPts-1, choosing a $\mathrm{k} \leq 6$ leads to detection of many fake groups with a small number of members. The best Eps value is the one where the k-dist plot shows a abrupt change in the slope. The physical meaning is such that all the objects on the right side of the chosen Eps value are considered as core points. Thus, choosing a very small Eps value leads to less detected structures whereas large Eps values tend to merge separate objects. We have examined all the possible Eps values with fixed MinPts=7 in the DBSCAN application and found that Eps=0.027 ( 270 kpc at redshift of A3888) is the value of interest and corresponds to structures with physical scales e.g.galaxy groups. Figure 6.7 shows the sorted 6-dist plot. 


\section{§6.6.2. Caustic Technique}

In order to use the information of peculiar velocity of the galaxies in our cluster member selection procedure, the caustic technique was used alongside the DBSCAN application. For this purpose, we used the CAUSTIC APP, an open source application initially developed by Ana Laura Serra (Serra, 2014).

Serra and Diaferio (2013) pointed out that "the caustic technique (Diaferio and Geller, 1997; Diaferio, 1999, 2009; Serra et al., 2011) identifies the escape velocity profile of galaxy clusters from their centre to radii as large as $3 R_{200} " 1$. This radius is considered as the point in the cluster outskirts right before the warm-hot intergalactic medium (WHIM) dominates the region (Reiprich et al., 2013). It is believed that the WHIM is the remaining hot gas from galaxy formation activities in the past (Reiprich et al., 2013).

The caustic method has been extensively employed to estimate the mass profile of galaxy clusters at distances much larger than the virial radius $\left(\approx R_{200}\right)$ (Regos and Geller, 1989; Diaferio, 1999; Rines and Diaferio, 2006; Serra et al., 2011). However, this method is also a valuable technique to trace the imprints of the gravitational potential well of the galaxy cluster at radii up to few megaparsecs.

As mentioned earlier, clusters lie at the knots of filaments of galaxies in the cosmic web. Since gravity is a long-range acting force, galaxies in much larger distances of the order of $10 \mathrm{Mpc}$ will still be affected by the gravity of the cluster (Diaferio and Geller, 1997). During mass accretion in the outskirts of the clusters, galaxies are accelerated by the cluster gravitational potential and consequently fall into the cluster. The aforementioned galaxies ultimately become a gravitationally bound member of the parent cluster. This process creates a characteristic trumpet-shaped escape velocity profile/putative caustic profile for all the galaxies which are interacting with the gravitational potential well of the cluster (Kaiser, 1987; Regos and Geller, 1989; van Haarlem and van de Weygaert, 1993).

The principal assumption in the caustic method is that clusters are spherical and symmetric systems, however, the cluster's morphology does not affect the caustic profile. The amplitude of the caustics, $A(r)$, is a combination of escape velocity profile and the velocity anisotropy parameter, $\beta(r)$, which is given by:

$$
\beta(r)=1-\left(\left\langle v_{\theta}^{2}\right\rangle+\left\langle v_{\phi}^{2}\right\rangle\right) / 2\left\langle v_{r}^{2}\right\rangle
$$

where $v_{\theta}, v_{\phi}$ and $v_{r}$ are longitudinal, azimuthal and radial component of the galaxy velocity in the volume $d r^{3}$ at position $r$ (Diaferio and Geller, 1997). The average squared

\footnotetext{
${ }^{1} R_{200}$ is defined as the radius of a sphere where the average density is 200 times the current critical density of the Universe.
} 
velocity of galaxies in a sphere with radius $r$ is:

$$
\left\langle v^{2}\right\rangle=\left\langle v_{L O S}^{2}\right\rangle g(\beta)
$$

where $v_{L O S}$ is the line of sight component of the galaxy velocity and

$$
g(\beta)=\frac{3-2 \beta(r)}{1-\beta(r)} .
$$

The escape velocity at the radius $r$ is $\left\langle v_{\text {esc }}^{2}(r)\right\rangle=-2 \phi(r)$ where $\phi(r)$ is the gravitational potential. If we assume that the caustic amplitude, $A(r)$, is the LOS component of the escape velocity $A^{2}(r)=\left\langle v_{e s c, L O S}^{2}\right\rangle$ then we have:

$$
-2 \phi(r)=A^{2}(r) g(\beta) \equiv \phi_{B}(r) g(\beta)
$$

This equation shows that the caustic amplitude, $A(r)$, is related to the potential well of the cluster .

To estimate $A(r)$, three major steps should be followed (Serra et al., 2011):

1. Construction of a hierarchical tree based on the calculation of projected binding energy for each pair of galaxies, presumed to have identical mass.

2. Determining a threshold to terminate the growth of the hierarchical tree and find the candidate cluster members.

3. Calculating the cluster centre, galaxy number density, caustic profile and determining optimal cluster members.

At first, the projected pair-wise binding energy of all of the galaxies pairs are used to create a hierarchical tree. In the second step, a threshold should be determined to cut the tree. Diaferio (1999) explained that in the hierarchical tree, there is a main branch that starts from the root and links "nodes" being places where galaxies are hanging. Each group of galaxies hang from nodes that have a velocity dispersion $\sigma_{n}$. The distribution of velocity dispersions of the nodes shows a characteristic feature which is used to determine the cutting threshold. The velocity dispersion $\sigma_{n}$ begins to drop significantly due to less interloper contamination and then the velocity dispersion begins to flatten until it reaches a node associated with internal structures after which it shows a significant drop. Diaferio (1999) mentioned that most of the galaxies hang from node where the " $\sigma$ plateau" begins, are defined as candidate cluster members. Figure 6.8 shows the velocity dispersion of the nodes along the main branch of the hierarchical tree. The left square indicates the node where the potential cluster members hang. In the last step, the cluster centre and the galaxy density is calculated to estimate the LOS caustics. 
Sigma Plateau

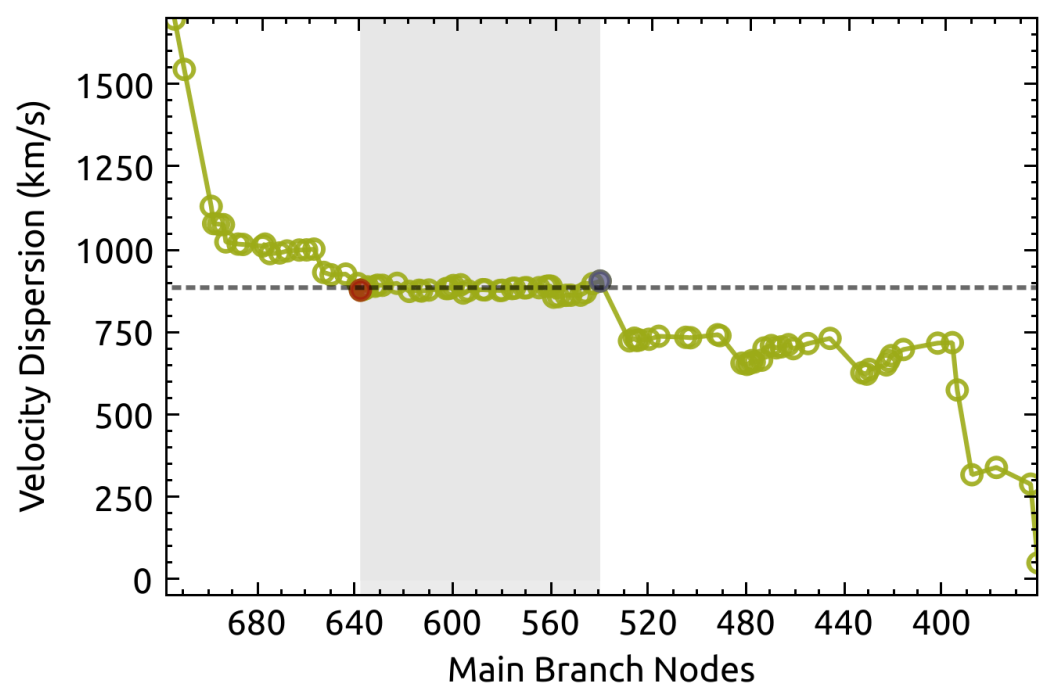

Figure 6.8: Velocity dispersion of the nodes along the main branch of the hierarchical tree for A3888. The first red square shows the node where the candidate cluster members hang. The shaded area represents the sigma-plateau in the velocity distribution.

Serra et al. (2011) stressed that the caustics are the values which satisfy the equation

$$
f_{q}(r, v)=\kappa
$$

where $f_{q}(r, v)$ is the calculated galaxy number density in $(\mathrm{r}, \mathrm{v})$ plane. The projected distance $r$ and LOS velocity $\mathrm{v}$ are given by

$$
r=\frac{c D_{A}\left(z_{c}\right)}{H_{0}} \sin \psi
$$

and

$$
v=c \frac{z-z_{c}}{1+z_{c}} .
$$

$\psi$ is the angular separation measured from the cluster centre to each galaxy, $D_{A}\left(z_{c}\right)$ is the angular distance of the cluster, and $\kappa$ is the root of the equation:

$$
\left\langle v_{\mathrm{esc}}^{2}\right\rangle_{\kappa, R}-4\left\langle v^{2}\right\rangle=0
$$

where $\left\langle v_{\mathrm{esc}}^{2}\right\rangle_{\kappa, R}=\int_{0}^{R} \mathscr{A}_{\kappa}^{2}(r) \varphi(r) \mathrm{d} r / \int_{0}^{R} \varphi(r) \mathrm{d} r$ is the mean caustic amplitude within the main group size $R, \varphi(r)=\int f_{q}(r, v) \mathrm{d} v$, and $\left\langle v^{2}\right\rangle^{1 / 2}$ is the velocity dispersion of the candidate cluster members, $\mathrm{R}$ is their mean projected distance to the centre, and $\mathrm{q}$ is the smoothing parameter used in the construction of the galaxy number density map. The actual clusters members are the galaxies which are located within the caustics. The caustic profile of A3888 is shown in Figure 6.9. Red dots represent the determined 


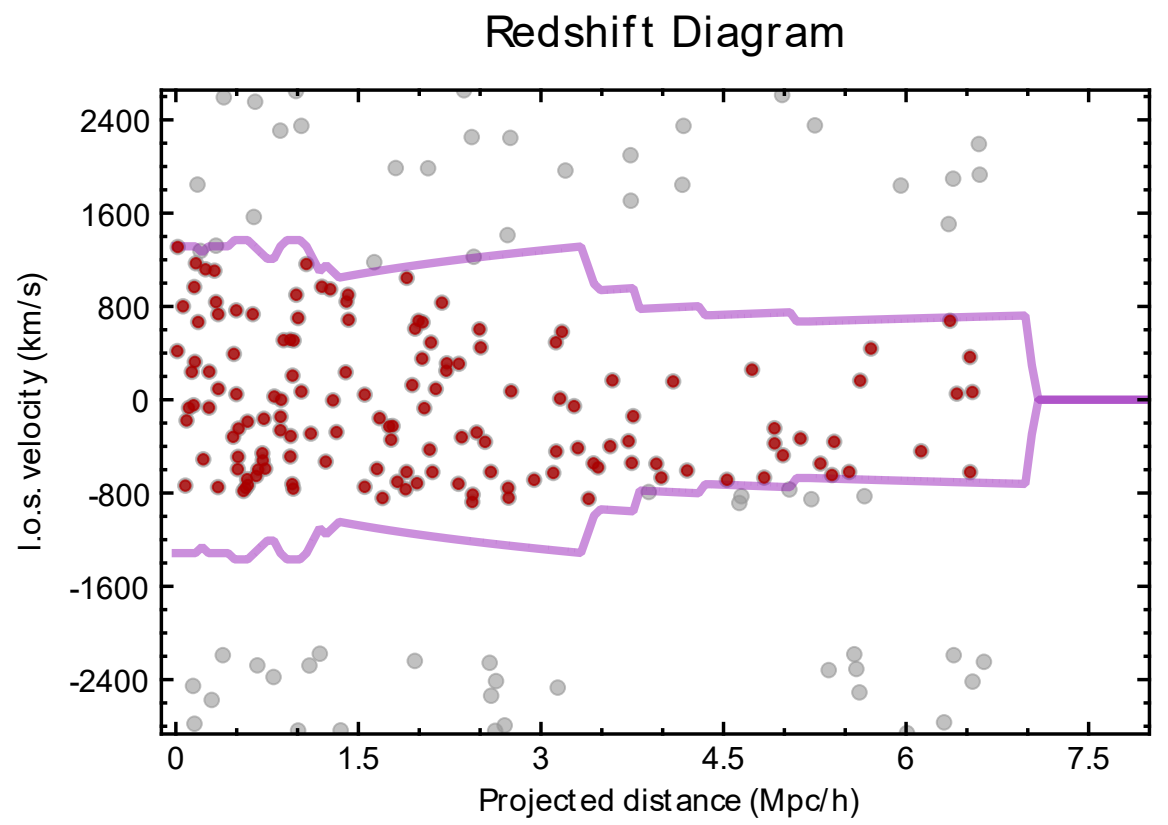

Figure 6.9: The caustic profile for A3888 (magenta solid lines). The red dots show the cluster members defined by the caustic technique. The gray dots are the interloper galaxies which are not gravitationally bound to A3888.

cluster members which reside within the caustics. The other galaxies in grey are not gravitationally bound to A3888.

As discussed earlier, the caustic technique measures the escape velocity profile which can be used as a cluster member allocation tool in the dynamical analysis of the clusters (e.g. Owers et al., 2013, Rines et al., 2013 and Owers et al., 2014 ). The caustic profile of clusters carry invaluable information about each of the galaxies in the field of cluster. The position of individual galaxies in the caustic profile reflects the time when infall inwards the cluster started. Galaxies which are passing the cluster core for the first time have higher caustic amplitudes due to acceleration whereas galaxies which have accreted at the same time that core of the cluster was forming, tend to be closer to the cluster centre and have lower caustic amplitudes (Haines et al., 2012). The results of the DBSCAN and caustic technique applications are presented in the following section.

\section{$\S 6.7$. Structures in the Main Population of A3888}

The colour-coded redshift histogram of the galaxies in a one degree radius around A3888, revealed that members of A3888 fall in the range of $0.12<\mathrm{z}<0.0185$. In $\S 6.6$, we explained how the DBSCAN and caustic techniques were used to constrain the interlopers in the galaxy member selection process. These methods allowed us to utilise both redshift and position information to determine the most probable member candidates of A3888.

Firstly, the DBSCAN method was applied to the data in the redshift range 0.12 - 
0.0185 and consequently, 77 candidate galaxy members were identified. Further refinement with the caustic method shrunk the number of cluster members to 67 galaxies. In order to verify that the final results were not affected by the order of application, the reverse order (caustic-DBSCAN) was also examined. In the reverse order, the caustic technique was first applied to the data and 146 galaxies were identified as member candidates of the cluster A3888. Then, the DBSCAN method was applied to the caustic method results and 65 galaxies were identified as the final candidate members of A3888 (see Figure 6.9). There were 61 galaxies in common in both orders of applications, four extra galaxies were detected in the caustic-DBSCAN order and six further galaxies in DBSCAN-caustic order. Because the caustic-DBSCAN order is more conservative than the reverse order (DBSCAN-caustic) we combined both results and consequently the total number of candidate galaxy members increased to 71 .

In addition, the DBSCAN method detected four other galaxy groupings in the redshift range of A3888. The isoline plot of the cluster A3888 and the four detected galaxy grouping are shown in Figure 6.10. In the following discussion, details of A3888 and other detected structures in redshift slice $0.12-0.0185$ by the DBSCAN are given and the five structures are also shown on Figure 6.10 as numbered groups of coloured points. We have used a method presented by Beers et al. (1990) to estimate the redshift and velocity dispersion of the detected structures. All the reported confidence intervals are calculated based on $\alpha=0.05$ (95\% confidence level). In Table 6.2 details of the detected groups such as the rest frame velocity dispersion, and observed redshift are given.

- Structure 1

This is the main cluster, A3888 with 71 members. The members are the combined resultant galaxies of caustic-DBSCAN and DBSCAN-caustic applications. The presence of an elongation towards the East of the cluster might be indicative of sub-clustering in A3888. The estimated average redshift and rest frame velocity dispersion of the cluster were calculated as 0.1535 and $1181 \mathrm{~km} \mathrm{~s}^{-1}$, respectively. Details of further substructure tests are given in $\S 6.8$.

- Structure 2

This is a galaxy group located in the far North of A3888 and has 7 candidate members. This group has a rest frame velocity dispersion of $338 \mathrm{~km} \mathrm{~s}^{-1}$ at a mean redshift 0.1406 . The caustic method showed that the galaxy members of this group are not located between the caustics of A3888. Thus, this group is not gravitationally bound to the cluster.

- Structure 3

This is a galaxy group in the North of cluster A3888 with 5 candidate members. The estimated mean redshift and rest frame velocity dispersion are 0.1411 and 


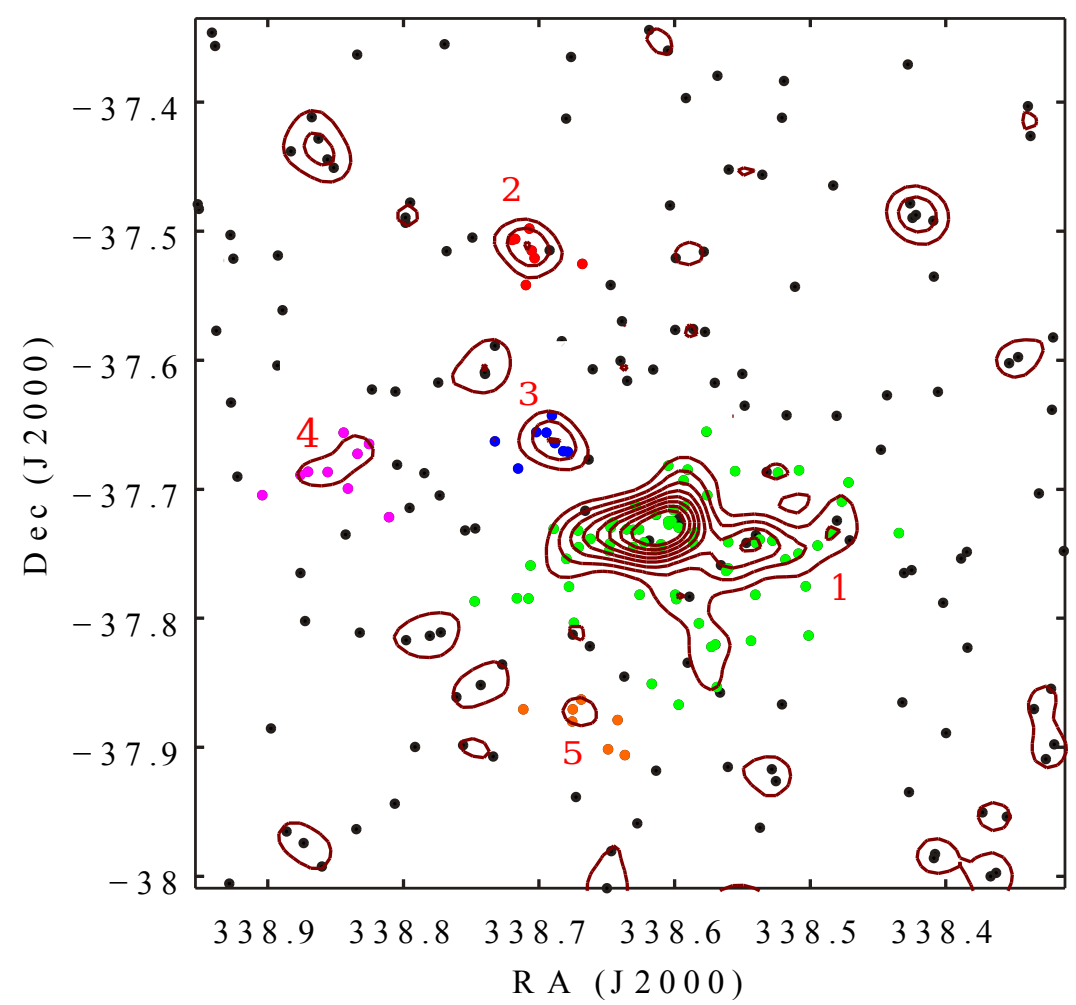

Figure 6.10: The isoline plot of the groups and cluster A3888 detected by DBSCAN method. The contours start at $10 \%$ and continues in $10 \%$ interval to $90 \%$. Green dots represent the main cluster A3888 and dots in red, blue, magenta and orange demonstrate the galaxy groups 2-5 respectively which found by DBSCAN method. See Table 6.2 for more details.

$164 \mathrm{~km} \mathrm{~s}^{-1}$, respectively. Using the caustic method revealed that the galaxies in this group are not gravitationally bound to the main cluster A3888.

- Structure 4

This structure with 9 members has two distinct velocity distributions and consequently, the rest frame velocity dispersion at mean redshift of 0.1529 was estimated as $1025 \mathrm{~km} \mathrm{~s}^{-1}$ which is considerably higher than the expected velocity dispersion of galaxy groups (400-500 $\mathrm{km} \mathrm{s}^{-1}$ ). As a result we believe this is not a real structure, but rather a fake grouping.

\section{- Structure 5}

This is a fake structure featuring a sporadic velocity distribution. There is no evidence of Gaussianity in the velocity distribution. This suggests that this detection is entirely contaminated by projection effects of galaxies which are distributed in a large redshift slice. The estimated mean redshift and rest frame velocity dispersion were 0.1603 and $3232 \mathrm{~km} \mathrm{~s}^{-1}$, respectively.

In Figure 6.11 the spatial distribution of the member galaxies of A3888 and the detected galaxy groups (structures 2 and 3 ) are shown. The background image is the DSS blue image. 
Table 6.2: Details of the detected galaxy groups and cluster A3888 in the main population. All of the detected structures are in the redshift range of $0.12-0.185$. The upper and lower limits of the redshift and rest frame velocity dispersion represent the estimated $\alpha=0.05,95 \%$ confidence intervals.

\begin{tabular}{ccccl}
\hline \hline Structure ID & $\mathrm{N}_{\text {gal }}$ & $\mathrm{z}$ & $\sigma_{v, \text { rest }}\left[\mathrm{km} \mathrm{s}^{-1}\right]$ & Comment \\
\hline 1 & 71 & $0.1535_{-0}^{+0.00009}$ & $1181_{-197}^{+197}$ & Cluster A3888 \\
2 & 7 & $0.1406_{-0.0011}^{+0.0011}$ & $338_{-126}^{+386}$ & Galaxy group \\
3 & 5 & $0.1411_{-0.0008}^{+0.0008}$ & $164_{-66}^{+305}$ & Galaxy group \\
4 & 9 & $0.1529_{-0}^{+0.0037}$ & $1025_{-402}^{+402}$ & Fake galaxy group \\
5 & 7 & $0.1603_{-0.0111}^{+0.0108}$ & $3232_{-1978}^{+301}$ & Fake galaxy group \\
\hline
\end{tabular}

Table 6.3: Details of the detected substructures in A3888.

\begin{tabular}{cccc}
\hline \hline Groups & $\mathrm{N}_{g a l}$ & $\mathrm{z}$ & $\sigma_{v, \text { rest }}\left[\mathrm{km} \mathrm{s}^{-1}\right]$ \\
\hline $\mathrm{G} 1$ & 41 & $0.1505 \pm 0.0010$ & $853 \pm 203$ \\
$\mathrm{G} 2$ & 26 & $0.1566 \pm 0.0013$ & $784 \pm 207$
\end{tabular}

\section{§6.8. Substructure Test of A3888}

In Figure 6.12 the redshift histogram of the 71 final cluster member candidates is plotted. A departure from Gaussianity is evident around redshift 0.157 . In order to verify that this peak in the histogram is statistically significant, we performed a Lee-Fitchett (Lee, 1979; Fitchett, 1988) 3D substructure test using the CALYPSO package (Dehghan \& Johnston-Hollitt in prep). The substructure test was carried out by Dr Siamak Dehghan who kindly provided the results to us. A clipping procedure in the software has automatically rejected four of our 71 candidate member galaxies. According to the substructure test result, A3888 is bimodal. The substructure significance was $99.8 \pm 0.2 \%$ (99.00\% confidence level) which is equivalent to a 3.16-sigma detection. However, due to the relatively low redshift sampling of the region, this result is likely subject to change as more redshifts become available in the future.

Figure 6.13 shows the results of the Lee-Fitchett 3D test for A3888. The detected subgroups named G1 and G2 have average redshifts of 0.1505 and 0.1566 , respectively. The rest frame velocity dispersion of G1 and G2 were measured as $853 \pm 203$ and $784 \pm 207 \mathrm{~km} \mathrm{~s}^{-1}$ at $95 \%$ confidence level. Notably the cluster is split into the two subgroups along the same east-west axis as the overall density elongation. Table 6.3 gives the properties of the two subgroups detected here. We stress that the cumulative redshift coverage is only up to $30 \%$ within $1.5 \mathrm{Mpc}$ radius around the cluster where the 71 candidate members reside. Thus, this substructure test is subject to change with improved spectroscopic redshift sampling of the region. 


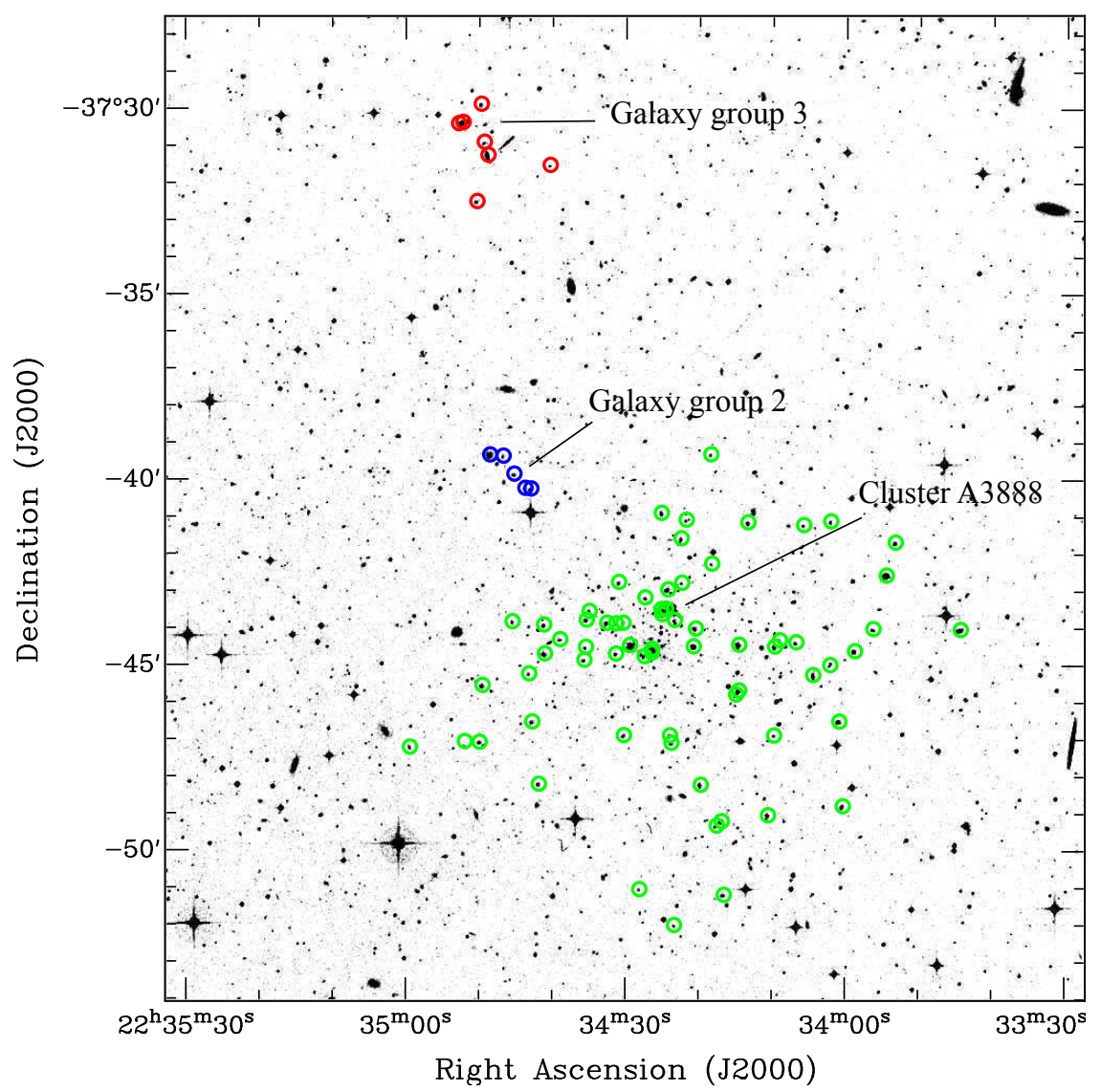

Figure 6.11: Distribution of member galaxies of cluster A3888 and the two detected groups (Structures 2 \&3). A3888 and galaxy groups 2 and 3 are demonstrated with green, blue and red open circles, respectively, on the DSS blue image.

\section{§6.9. Foreground and Background Structures}

As mentioned earlier, according to the colour-coded histogram (see Figure 6.4), there are other fore/background populations of galaxies in a one degree radius around A3888. The DBSCAN method was applied to all of the separate populations distributed in the redshift histogram. According to the DBSCAN results, galaxy populations in the lightblue $(0.053 \leq \mathrm{z} \leq 0.0875)$ and red $(0.185 \leq \mathrm{z} \leq 0.222)$ regions in the colour-coded histogram (Figure 6.4) host galaxy clusters and galaxy groups.

Examination of the DSS blue image revealed existence of three $\mathrm{cD}$ galaxies in the field with conspicuous mass concentrations around them. Interestingly, the application of the DBSCAN to galaxies in the redshift range $0.053 \leq \mathrm{z} \leq 0.0875$ (the light blue grouping on Figure 6.4) confirmed the existence of three galaxy clusters around the aforementioned $\mathrm{cD}$ galaxies. Thus, the DBSCAN results were consistent with the visual inspection of the DSS blue image and hereafter these three clusters are denoted as Structures 6,7 and 8. Examination of the redshifts between 0.185 and 0.222 (red population on Figure 6.4) showed the presence of an additional galaxy group, denoted here as Structure 9. Table 6.4 gives the summary of the DBSCAN results on these over 


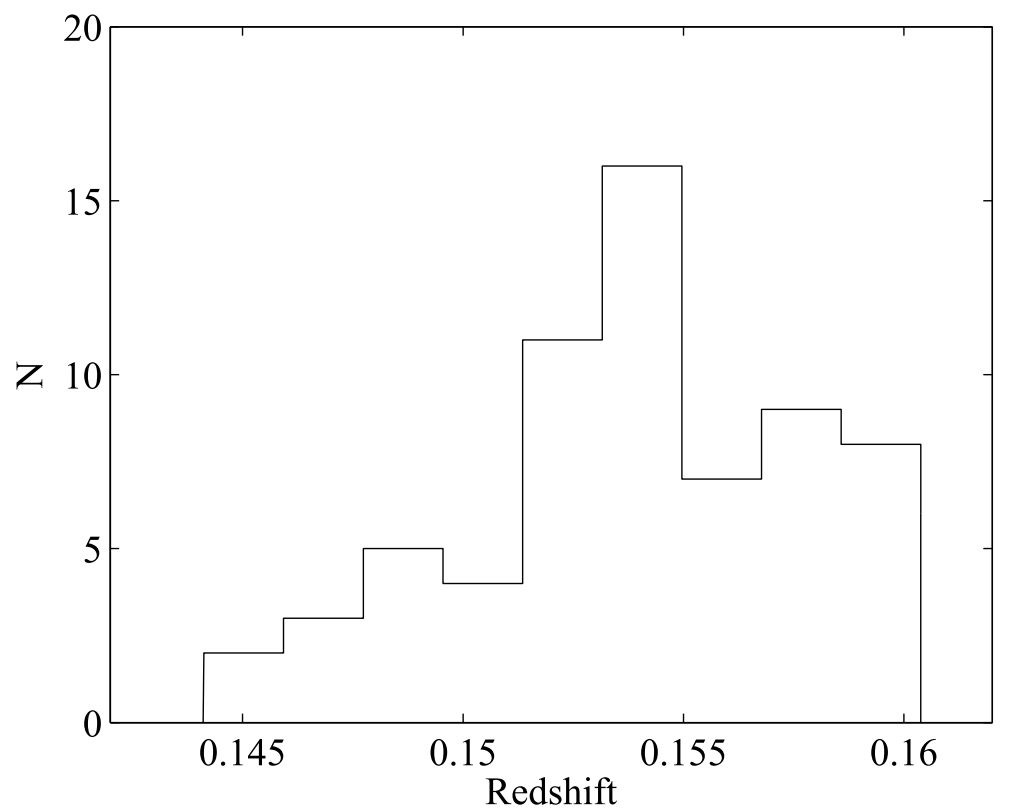

Figure 6.12: Redshift histogram of the 71 possible members of A3888 shown with a bin width of $540 \mathrm{~km} \mathrm{~s}^{-1}$. A departure from Gaussianity is evident around $\mathrm{z} \sim 0.157$.

Table 6.4: Details of fore/back ground structures detected by the DBSCAN method in the field of A3888. The upper and lower limits of the redshift and velocity dispersion represent the estimated $\alpha=0.05,95 \%$ confidence intervals.

\begin{tabular}{ccccccc}
\hline \hline Structure ID & $\mathrm{z}_{c D}$ & $\mathrm{~N}_{g a l}$ & $\mathrm{z}$ & $\sigma_{v, \text { rest }}\left[\mathrm{km} \mathrm{s}^{-1}\right]$ & $\mathrm{cD}$ coord \\
\hline 6 & 0.0795 & 17 & $0.0792_{-0.0013}^{+0.0013}$ & $730_{-363}^{+363}$ & $22: 32: 35$ & $-37: 42: 28.93$ \\
7 & 0.0741 & 24 & $0.0765_{-0.0022}^{+0.0022}$ & $727_{-592}^{+592}$ & $22: 32: 49$ & $-37: 59: 24.91$ \\
8 & 0.0733 & 7 & $0.0732_{-0.0005}^{+0.0005}$ & $128_{-19}^{+209}$ & $22: 31: 07$ & $-38: 01: 35.95$ \\
9 & $\ldots$ & 6 & $0.2006_{-0.0020}^{+0.0020}$ & $420_{-175}^{+541}$ & $22: 34: 16$ & $-37: 19: 25.01^{\mathrm{a}}$ \\
\hline
\end{tabular}

${ }^{a}$ The coordinate of the brightest galaxy.

densities and further narrows the ranges over which the structures occur. As mentioned earlier, because of poor redshift coverage, determining all of the members of the detected clusters and galaxy groups is not feasible and all the analysis is based on the available limited measured redshifts. As before, we used the method of Beers et al. (1990) for the calculations of velocity dispersions and redshifts of the detected structures. All of the confidence intervals were estimated based on $95 \%$ confidence level $(\alpha=0.05)$.

- Structure 6

This structure is a galaxy cluster which has 17 candidate members. The cD galaxy has a redshift of 0.0795 and is denoted as cD galaxy 1 on Figure 6.14. All of the candidate members of this cluster are annotated with blue open circles on the DSS 

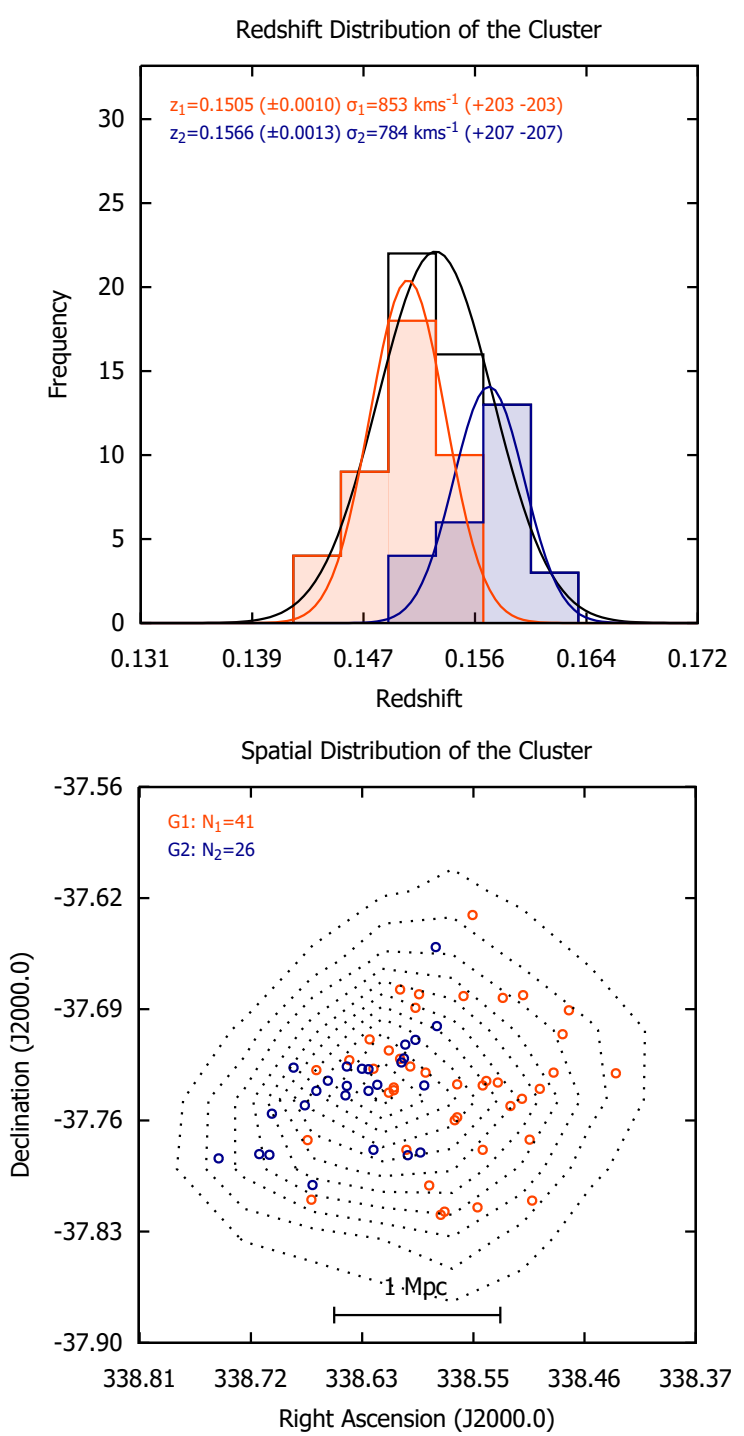

Figure 6.13: Results of the Lee-Fitchett 3D substructure test of A3888. The cluster is bimodal and the two subgroups are denoted as G1 (in red) and G2 (in blue). Left plot: histogram of all the members, G1, and G2 are shown with black, red, and blue lines, respectively. The estimated redshift and rest frame velocity dispersion for G1 and G2 are given in red and blue, respectively. Right plot: Spatial distribution of the detected groups G1 and G2. The number of candidate members of G1 and G2 are shown in red and blue, respectively.

blue image in Figure 6.14. The estimated average redshift and rest frame velocity dispersion of the cluster are 0.0792 and $730 \mathrm{~km} \mathrm{~s}^{-1}$, respectively. The contour plot of this structure is shown in Figure 6.15 (blue dots with label 6). In the left plot in Figure 6.16 the redshift histogram of this cluster is shown. We stress that the velocity dispersion might have been underestimated because of poor sampling in this region.

- Structure 7

This structure is a galaxy cluster which has 24 candidate members with a cD galaxy with redshift of 0.0741 which is denoted as cD galaxy 2 on Figure 6.14. 


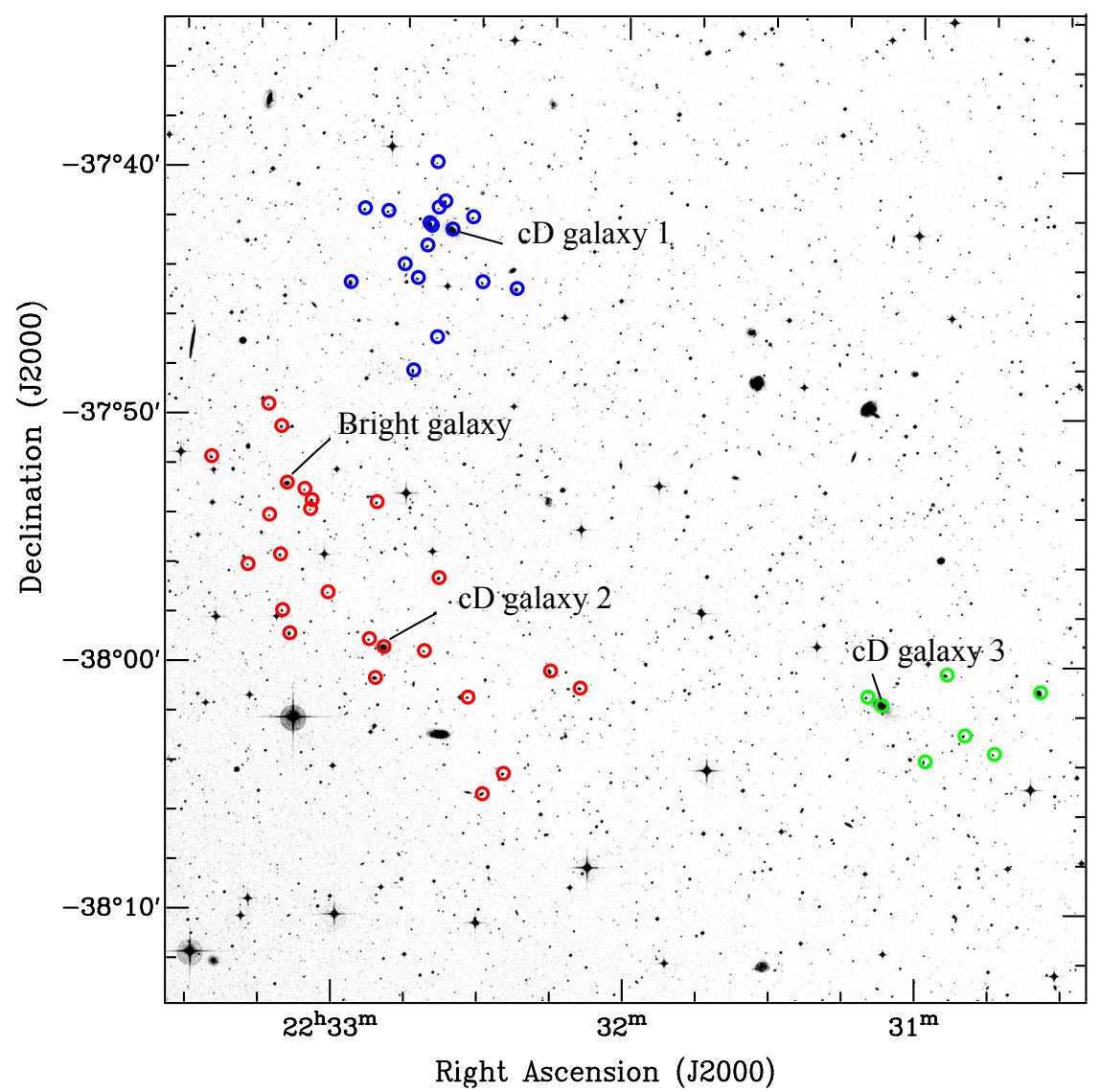

Figure 6.14: The spatial distribution of galaxies in the detected structures. The cD galaxies of detected galaxy clusters are annotated on the plot. Structure 6, 7 and 8 are colour-coded in blue, red and green, respectively. The DSS blue is the background image.

The candidate members with red open circles are shown in Figure 6.14. The average redshift and the rest frame velocity dispersion of this cluster is 0.0765 and 727 $\mathrm{km} \mathrm{s}^{-1}$, respectively. The contour plot of this structure is shown in Figure 6.15 ( red dots with label 7 ). The middle plot in Figure 6.16 shows the redshift histogram of this cluster with two well-separated redshift distribution which might explain the large estimated intervals of the velocity dispersion. The left peak in the histogram is consistent with the redshift of the $\mathrm{cD}$ galaxy. Additionally, the right peak in the histogram is in line with the redshift of a bright galaxy $(\mathrm{z}=0.0786)$ which is probably the Brightest Cluster Galaxy (BCG) of an interacting cluster and is also denoted as "Bright galaxy" in Figure 6.14. The projected distance (estimated at the mean redshift of both galaxies) between these two galaxies is $695 \mathrm{kpc}$. Figure 6.17 shows the two populations of galaxies with different velocity distributions according to the histogram shown in middle plot in Figure 6.16. The redshift distribution might be a signpost of an dynamical interaction between a galaxy cluster and a group or another cluster of galaxies. Further analysis requires greater redshift sampling of this region. 


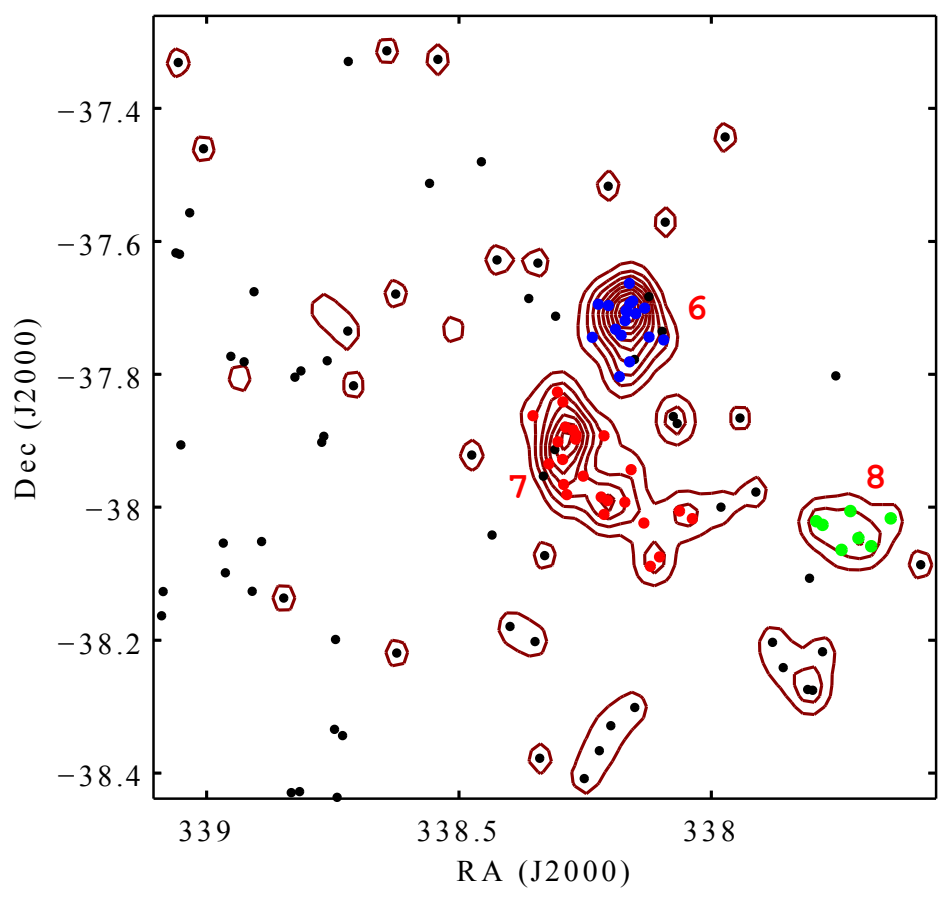

Figure 6.15: The isoline plot of the galaxies in redshift slice of $0.0717-0.0856$. The contour starts from $10 \%$ and continues with $10 \%$ interval to $90 \%$.

\section{- Structure 8}

This is a galaxy cluster with 7 members which has an average redshift of 0.0732 and hosts a cD galaxy with $\mathrm{z}=0.0733$ which is denoted as $\mathrm{cD}$ galaxy 3 in Figure 6.14. The candidate members are annotated with green open circles in Figure 6.14. The contour plot of this structure is shown in Figure 6.15(green dots with label 8). The right plot in Figure 6.16 shows the redshift histogram of this cluster. The redshift of the $\mathrm{cD}$ galaxy is in line with the peak of the histogram (Figure 6.16, right plot). On account of insufficient redshift coverage the rest frame velocity dispersion $\left(128 \mathrm{~km} \mathrm{~s}^{-1}\right)$ will be highly underestimated, but is sufficient for a group.

\section{- Structure 9}

There is another galaxy group found in the redshift slice of $0.1867-0.2162$. This is a very small group of merely six galaxies. The average redshift and rest frame velocity dispersion of this group are 0.2006 and $420 \mathrm{~km} \mathrm{~s}^{-1}$, respectively. The estimated velocity dispersion has a large confidence interval which is more likely to be a result of poor sampling. Figure 6.18, shows the distribution of galaxies in the detected galaxy group (Structure 9). The candidate member galaxies are indicated with the red open circles on the DSS blue image. Figure 6.19 shows the isoline plot of Structure 9 (blue dots with label 9) which is superimposed on the 


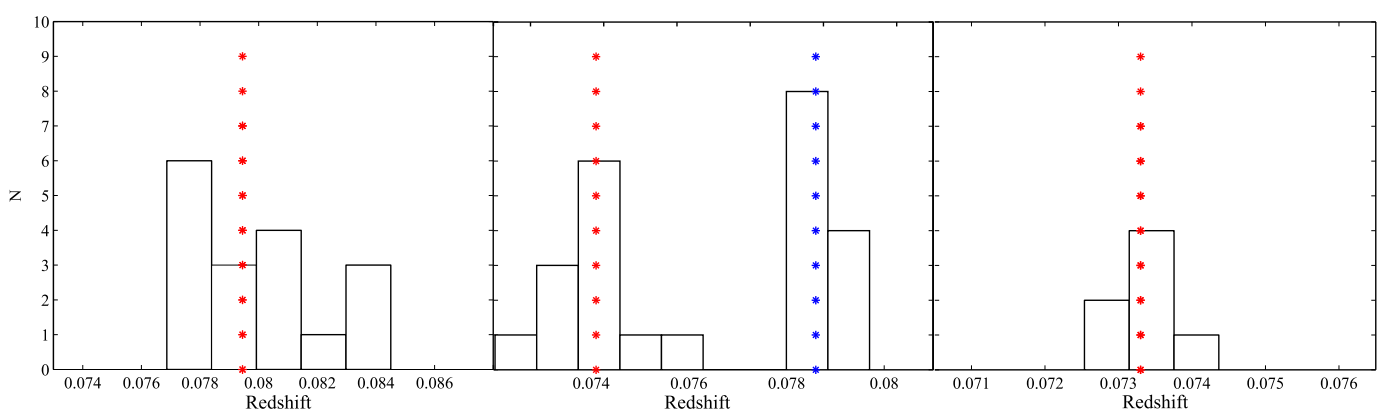

Figure 6.16: Redshift histogram of the clusters found in the redshift range $0.0717-0.0856$. The red vertical dotted line represents the redshift of the $\mathrm{cD}$ galaxy in each cluster. The blue dotted line represents the redshift of the BCG. The redshift histograms of Structures 6, 7 and 8 are shown from left to right, respectively.

scatter plot.

\section{$\S 6.10$. Morphological Content of A3888}

We have used the spectral atlas of galaxies presented by Kinney et al. (1996) to determine the morphology of the galaxies in our AAOmega sample throughout the redshift determination by RUNZ. All sets of ASCII templates cover the ultraviolet to near infrared spectral range with the wavelength range of $1235-9935 \AA$. These spectral templates are ideal to identify various galaxy morphologies from elliptical to late type spirals. The full details of the templates for different galaxy morphology are found in (Kinney et al., 1996). All of the spectra were visually inspected to confirm the galaxy morphologies determined by RUNZ. We have also used the spectrophotometric atlas of galaxies presented by Kennicutt (1992) to compare our spectra with the reference spectra provided in this atlas. We found that the majority of our observed galaxies across the field of AAOmega were late-type spirals $\left(S_{b}\right.$ and $\left.S_{c}\right)$. However, these spiral galaxies were mainly field galaxies scattered in various redshift slices. As it is expected, most of the early type galaxies were found in the over-dense regions particularly within a $1 \mathrm{Mpc}$ radius around the core of $\mathrm{A} 3888$.

Understanding the evolution of early/late type galaxies in clusters has been one of the major interests in optical astronomy. Recent large spectroscopic surveys (see $\S 6.1$ ), have provided an ideal opportunity to explore the morphological content of galaxy clusters and probe their evolution. For instance, Goto et al. (2003) studied 514 galaxy clusters in the redshift range $0.02<\mathrm{z}<0.3$ from the SDSS. According to their findings, the fraction of blue galaxies at the redshift of A3888 ( $\mathrm{z}=0.1535)$ should be around $15 \%$. Later on, Poggianti et al. (2009) investigated the correlation between morphological content of clusters with the cluster's global properties such as X-ray luminosity and velocity dispersion. They studied the Wide-Field Nearby Galaxy-cluster Survey clusters 


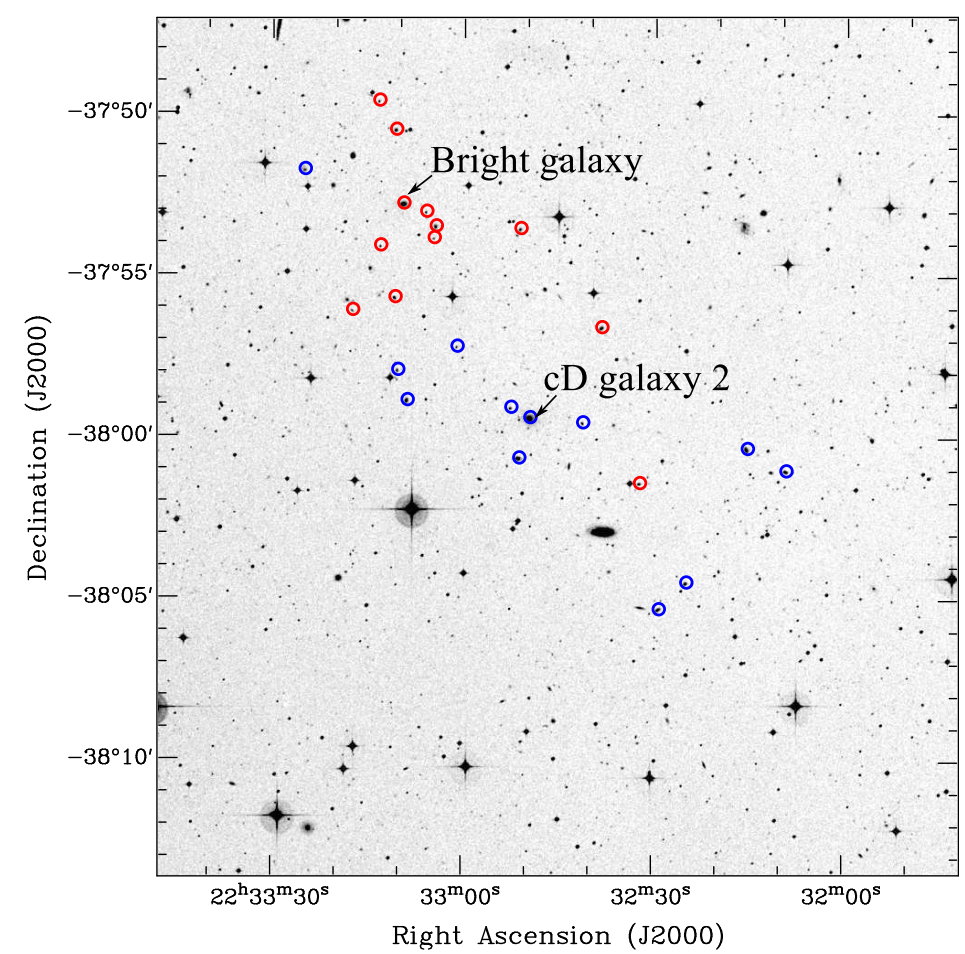

Figure 6.17: Redshift distribution of Structure 7 based on the velocity gap in the redshift histogram (see the middle plot in Figure 6.16). Blue circles represent the galaxy population of the left side of the histogram in redshift range of 0.073-0.077 and red circles demonstrate the galaxies in the right side of the histogram in redshift range of $0.077-0.080$. The DSS blue is the background image.

(WINGS) extensively to examine the effect of cluster properties and environment on the evolution of different galaxy morphology in clusters. WINGS is a multi wavelength photometric and spectroscopic survey of 77 galaxy clusters at $0.04<\mathrm{z}<0.07$ (Fasano et al., 2006). They additionally used 15 high-redshift clusters in former publications to accurately quantify any observed correlation. Poggianti et al. (2009) found that in general, at lower redshifts the morphological content of galaxy clusters are 23:44:33 corresponding to spiral, S0 and elliptical galaxies respectively. Moreover, they found that the spiral fraction of galaxy clusters, inversely correlates with the X-ray luminosity of the parent cluster.

To expand our optical analysis of A3888, we have investigated the morphological content of A3888 and also verified the location of A3888 with respect to current proposed correlations between the morphological segregation in clusters and the global properties of the parent cluster. Since the majority of redshifts of candidate members of A3888 come from the spectroscopic observations in the LARCS project (Pimbblet et al., 2006), we used the B-R colour reported in the LARCS spectroscopic catalogue to derive the spectral typing of the galaxies in our final redshift sample. This treatment has allowed us to supplement our AAOmega spectral typing information to determine the morphological fraction of the cluster A3888. Pimbblet et al. (2006) reported that 


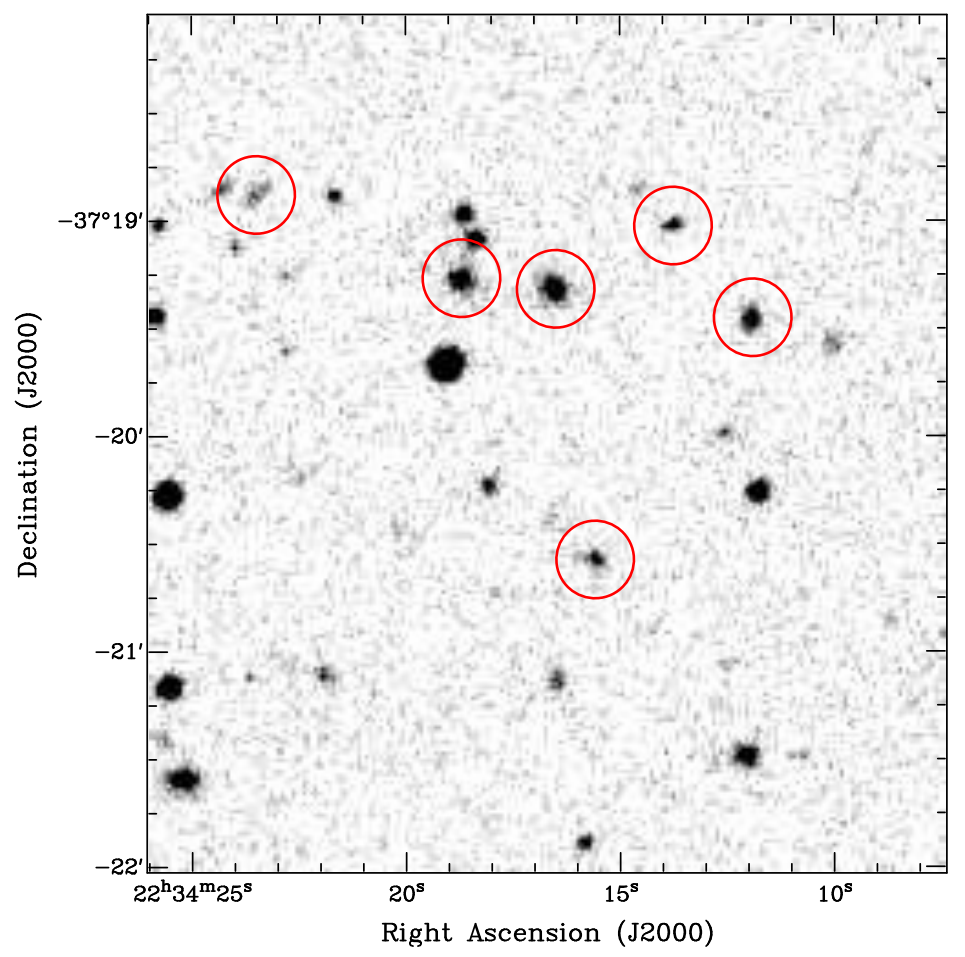

Figure 6.18: Distribution of galaxies in structure 9 found by the DBSCAN method.

the colour of the emission and absorption line galaxies are $(\mathrm{B}-\mathrm{R})<1.6$ and $(\mathrm{B}-\mathrm{R})>2$ respectively in their sample, thus galaxies with B-R colour between 1.6 and 2 could be either an absorption or an emission line.

In total, the 71 candidate members of the cluster were comprised of 9 emission line galaxies, 43 absorption line galaxies, 7 galaxies with $1.6<\mathrm{B}-\mathrm{R}<2$ in the LARCS catalogue and the remaining 12 members had no morphological or spectral typing data available in the literature. Thus of the 52 galaxies with confirmed morphological types, $\sim 17 \%(9 / 52)$ are emission line galaxies and $\sim 83 \%$ (43/52) are absorption line objects. Additionally, for the 19 remaining galaxies (12 without morphological information and 7 with $1.6<\mathrm{B}-\mathrm{R}<2$ ) one could assume these could be $100 \%$ emission or absorption line to derive the upper and lower limits of morphological content of the cluster. According to these assumptions the estimated emission and absorption fractions are $0.17_{0.13}^{0.40}$ and $0.83_{0.60}^{0.88}$, respectively. In Figure 6.20, the two top plots show the correlation between the $\mathrm{X}$-ray luminosity of the parent cluster and the morphological content of the cluster as the fraction of galaxy morphological type in (Poggianti et al., 2009). The location of A3888 is marked with a red filled dot with errors from the limits of the morphological fraction described above. The bottom plots indicate the correlation between the rest frame velocity dispersion and the morphological fraction of the clusters. As can be seen from the plot, the morphological content of A3888 is in agreement with the results derived by Poggianti et al. (2009). Although our redshift coverage is not similar to aforementioned galaxy cluster surveys, our results suggest that the morphological fraction of galaxies in 


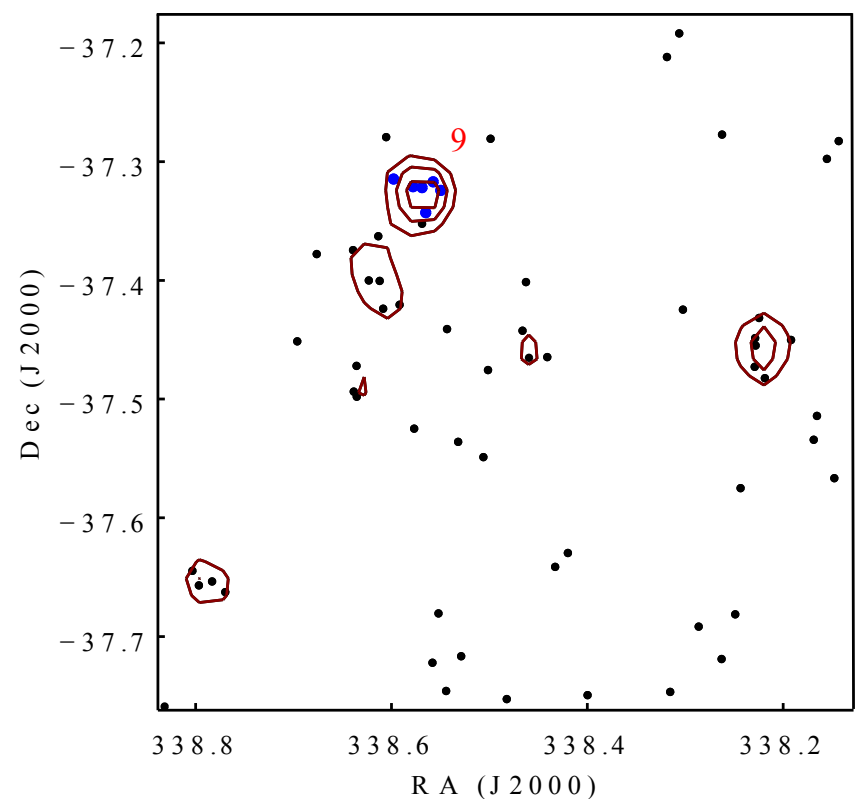

Figure 6.19: Contour plot of Structure 9. The contour levels are 50\%, 70\% and 90\%.

A3888 are in line with the predicted values in large surveys.

\section{§6.10.1. Radio/Optical Connection Investigation}

As we mentioned earlier in $\S 5.1$, we confirmed a GRH in A3888 and the major reason for the optical observation of this cluster was to provide a more clear insight into the dynamical activity of the cluster from optical perspective. In Figure 6.21, we compare the radio and optical results. In this plot, positions of the possible member galaxies determined in the optical analysis of A3888 are marked with blue circles. Black contours indicate the low resolution radio emission of the radio halo at $1.4 \mathrm{GHz}$. Red crosses present the position of the radio sources in the high-resolution radio image. As it is expected, the radio halo follows the distribution of the galaxies embedded in the ICM.

\section{$\S 6.11$. Summary and Conclusion}

In this chapter the results of optical observations of the cluster A3888 with the AAOmega spectrograph were presented. Cluster membership was carried out in three stages using the velocity and the position of the galaxies. Firstly, the velocity distribution of all galaxies with available redshift were examined and initial members were allocated based on the gap in the histogram around the mean redshift of the cluster (0.1535).

Further interloper rejection was performed using two different methods. The DBSCAN algorithm was used for the substructure analysis and galaxy member selection. The main cluster with 71 candidate member galaxies and two neighbouring groups were 

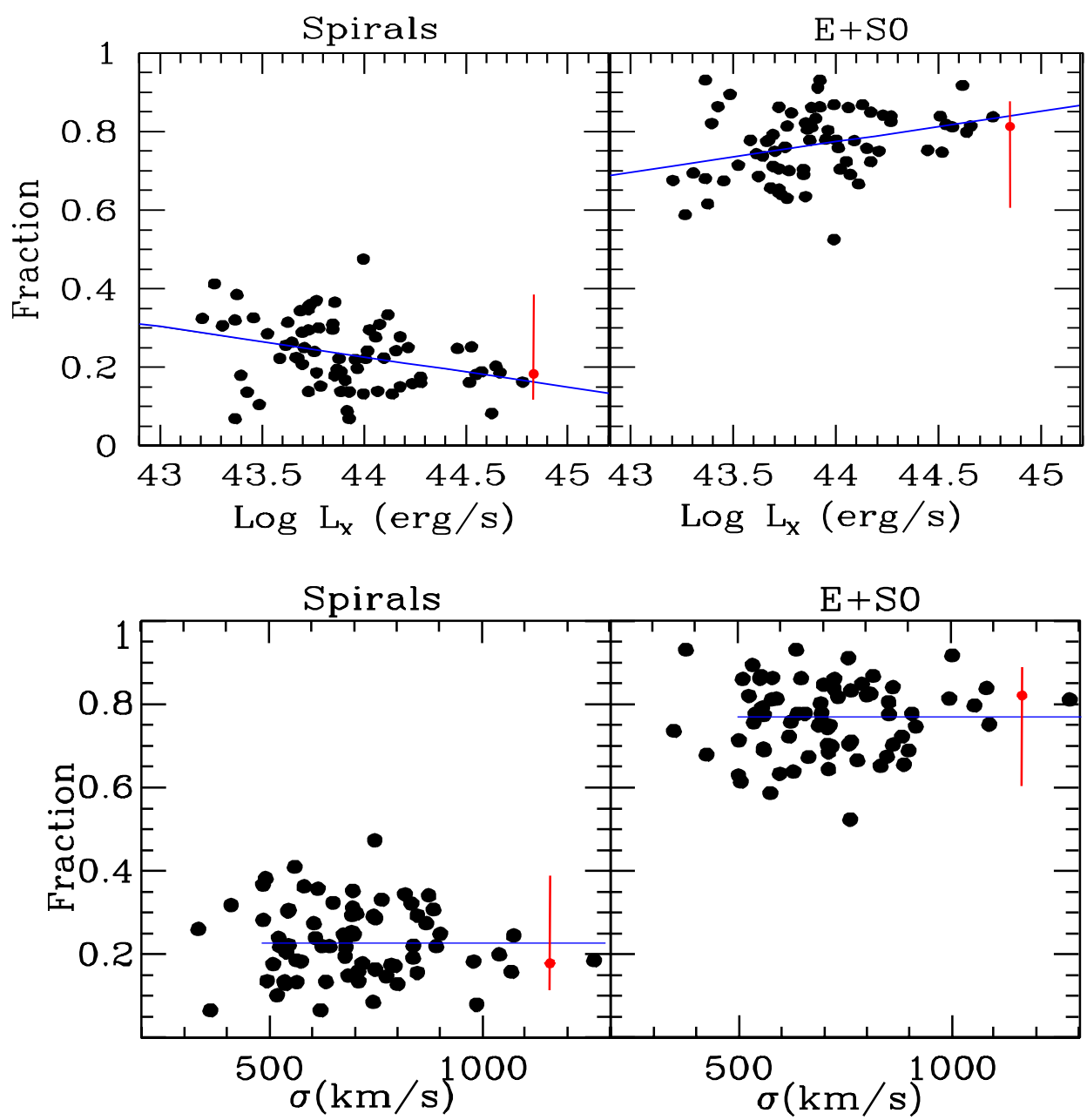

Figure 6.20: Top panels: the morphological fraction correlation with the X-ray luminosity of the parent cluster is shown with blue line (Poggianti et al., 2009). Bottom panels: the morphological fraction correlation with the cluster's rest frame velocity dispersion is shown with blue line (Poggianti et al., 2009). In all the plots, the location of A3888 is annotated with the filled red dot. The images are adapted from Poggianti et al., 2009 and the permission has received by private correspondence.

detected in the DBSCAN results. The caustic technique was also used for further membership refinement. We used the results of the CALYPSO package developed by Dr Siamak Dehghan which provided evidence of bi-modality in A3888. However, we note that due to poor redshift coverage our substructure test might not be robust and the result is prone to significant change once the redshift coverage enhanced in the future.

The contradictory evidence about the dynamics of A3888 from X-ray data explained in $\S 5.1 .4$ underlines that the dynamical staus of this puzzling cluster is not clear. However, the combination of pieces of evidence from the optical analysis such as the presence of multiple BCGs, the elongated optical galaxy distribution, and our substructure test which showed that A3888 has optical substructures suggests that this cluster has dynamical interactions. In addition, the latest X-ray morphology reported by Weißmann et al. (2013) confirmed that A3888 has local X-ray substructures and the large 


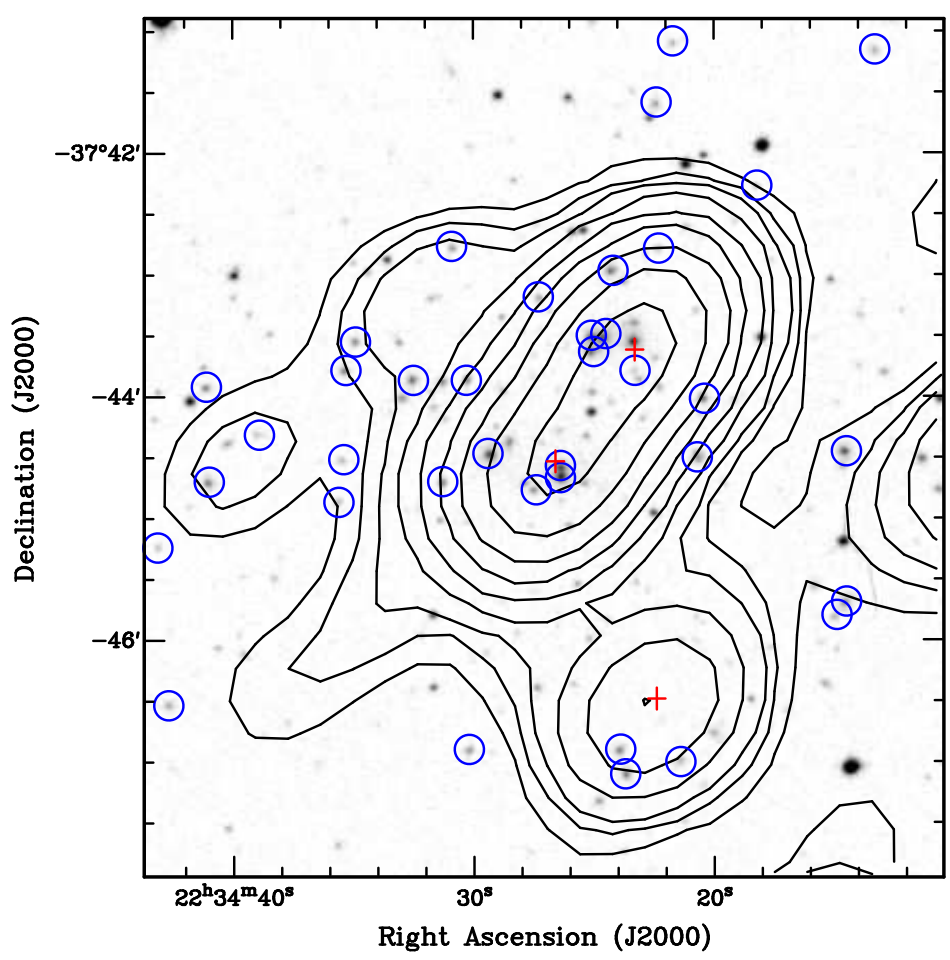

Figure 6.21: Distribution of possible member galaxies of A3888 are marked and the radio and Xray contours are overlaid on the background DSS blue image. Open blue dots represent possible member galaxies of A3888. black contour is the radio emission in the low resolution image and red crosses represent the radio objects in the high-resolution radio image. The background image is DSS blue. The contours start at $360 \mu \mathrm{Jy}_{\text {beam }}{ }^{-1}$ and are spaced in intervals increasing by a factor of 2 .

BCG/X-ray peak separation could confirm that A3888 is highly likely a young cluster in an active merging state.

According to the DBSCAN results, three galaxy clusters and a galaxy group were also detected in the foreground and background redshift slices. The morphological content of the cluster was studied and the results revealed that A3888 is a typical cluster where the majority of members are absorption line galaxies.

On account of the very small angular separation of the galaxies in the core of the cluster, single slit spectroscopy is required to increase the spectroscopic coverage. In particular, the $2.3 \mathrm{~m}$ telescope of the Australian National University (ANU) at Siding Spring Observatory with rotating slit could be used in the future to cover two or more galaxies in a single exposure (Fleenor et al., 2006). This would allow a more detailed probe of the cluster core and better statistics on the merging populations. 


\section{§7. Radio Halo Statistics: Scaling Relations and Bi-modality}

\section{$\S 7.1$. Introduction}

As discussed earlier in Chapter 1 and $\S 4.1$, giant radio halos (GRHs) are referred to as Mpc-scale synchrotron emission regions and are thought to be generated by reaccelerated electrons in a cluster's magnetic field. In the last decade, the number of detected halos has increased and the connection between the halo occurrence and the cluster's dynamical and environmental properties have been investigated. Despite significant development in theoretical explanations of the nature of radio halos, there has been little progress in proving halo generation scenarios (see $\S 2.7$ ). This is mainly due to the small number of halos, detected so far. In conjunction with the number of individual halo detections in the last decade, Venturi et al. $(2007,2008)$ conducted one of the first major radio halo surveys, in which they observed a sub-sample of the REFLEX and extended ROSAT Brightest Cluster Sample (eBCS) catalogue (Ebeling et al., 1998). They built a complete $X$-ray flux limited (up to redshift 0.3) sample of 47 galaxy clusters with high X-ray luminosity $\left(\mathrm{L}_{X}[0.1-2.4 \mathrm{keV}]>5 \times 10^{44} \mathrm{erg} \mathrm{s}^{-1}\right)$ within the redshift range of $0.2-0.4$, and carried out radio observations at $610 \mathrm{MHz}$ with the GMRT. They detected four GRHs and two halo candidates, which were all present in disturbed clusters.

Brunetti et al. (2007) used a novel approach to investigate the connection between radio halo occurrence and dynamical activity of all the clusters in the GRHS. They injected fake radio halos in the non-halo clusters using a well-studied integrated brightness profile of radio halos (Cassano et al., 2007) and investigated the location of nondetections on the $\mathrm{P}_{1.4}-\mathrm{L}_{X}$ plane. Intriguingly, they observed a "bi-modality" in the distribution of the clusters with/without halos. They claimed that the population of clusters with halos was well separated from the non-detection clusters in the $\mathrm{P}_{1.4}-\mathrm{L}_{X}$ plane. More recently, Kale et al. (2013) performed the Extended GMRT Radio Halo Survey (EGRHS) in which, an extra 17 galaxy clusters with the same selection criteria as GRHS were observed with the GMRT at $610 \mathrm{MHz}$, bringing the total to 64 systems. They stressed that the bi-modality was evident in their work.

The origin of bi-modality has been discussed in several papers. For instance, Brunetti et al. (2007) suggest that this bi-modal behaviour can be a signpost of the origin of radio halos and can be suggestive of the importance of merging in the occurrence of the 
Table 7.1: REXCESS sub-sample properties and dynamical information based on the soft X-ray band analysis in the $[0.1-2.4] \mathrm{keV}$ band.

\begin{tabular}{ccccccc}
\hline \hline Cluster & Abell Name & $z$ & $L_{X}[0.1-2.4]$ & $M_{500}$ & Morphology & GRH \\
\hline$R X C J 0006.0-3443$ & $\mathrm{~A} 2721$ & 0.1147 & 1.875 & $3.95_{-0.12}^{+0.12}$ & $\mathrm{I}$ & $\times$ \\
$R X C J 0049.4-2931$ & $\mathrm{~S} 0084$ & 0.1084 & 1.503 & $1.62_{-0.04}^{+0.04}$ & $\mathrm{R}$ & $\times$ \\
$R X C J 0145.0-5300$ & $\mathrm{~A} 2941$ & 0.1168 & 2.005 & $4.37_{-0.08}^{+0.08}$ & $\mathrm{I}$ & $\times$ \\
$R X C J 0211.4-4017$ & $\mathrm{~A} 2948$ & 0.1008 & 0.798 & $1.00_{-0.02}^{+0.02}$ & $\mathrm{R}$ & $\times$ \\
$R X C J 0225.1-2928$ & $\ldots$ & 0.0604 & 0.408 & $0.96_{-0.04}^{+0.04}$ & $\mathrm{I}$ & $\times$ \\
$R X C J 0345.7-4112$ & $\mathrm{~S} 0384$ & 0.0603 & 0.495 & $0.97_{-0.02}^{+0.02}$ & $\mathrm{R}$ & $\times$ \\
$R X C J 0616.8-4748$ & $\ldots$ & 0.1164 & 1.597 & $2.70_{-0.05}^{+0.06}$ & $\mathrm{I}$ & $\times$ \\
$R X C J 0645.4-5413$ & $\mathrm{~A} 3404$ & 0.1644 & 7.139 & $7.38_{-0.14}^{+0.14}$ & $\mathrm{I}$ & $\times$ \\
$R X C J 1236.7-3354$ & $\mathrm{~A} 0700$ & 0.0796 & 0.749 & $1.33_{-0.02}^{+0.02}$ & $\mathrm{I}$ & $\times$ \\
$R X C J 2014.8-2430$ & $\ldots$ & 0.1538 & 9.157 & $5.38_{-0.07}^{+0.07}$ & $\mathrm{R}$ & $\times$ \\
$R X C J 2023.0-2056$ & $\mathrm{~S} 0868$ & 0.0564 & 0.411 & $1.21_{-0.03}^{+0.03}$ & $\mathrm{I}$ & $\times$ \\
$R X C J 2129.8-5048$ & $\mathrm{~A} 3771$ & 0.0796 & 0.767 & $2.26_{-0.06}^{+0.06}$ & $\mathrm{D}$ & $\times$ \\
$R X C J 2149.1-3041$ & $\mathrm{~A} 3814$ & 0.1184 & 2.117 & $2.25_{-0.03}^{+0.03}$ & $\mathrm{R}$ & $\times$ \\
$R X C J 2218.6-3853$ & $\mathrm{~A} 3856$ & 0.1411 & 3.516 & $4.92_{-0.11}^{+0.11}$ & $\mathrm{R}$ & $\times$ \\
$R X C J 2234.5-3744$ & $\mathrm{~A} 3888$ & 0.151 & 6.363 & $7.36_{-0.09}^{+0.09}$ & $\mathrm{I}$ & $\checkmark$ \\
\hline
\end{tabular}

Columns: (1) Cluster name; (2) cluster Abell name ; (3) (z): cluster redshift; (4) $L_{X}[0.1-2.4]$ (keV band) (Böhringer et al., 2010) X-ray luminosity in $R<R_{500}$ region in units of $10^{44} \mathrm{erg} \mathrm{s}^{-1}$ (Böhringer et al., 2010); (5) $M_{500}$, cluster mass; (6) dynamical status of the cluster based on the paper presented by Weißmann et al. (2013), R: relaxed, I: intermediate, D: disturbed cluster with double sub-structures; (6) shows if the cluster hosts a GRH.

radio halos in clusters. Soon after, Brunetti et al. (2009) and Cassano et al. (2011) mentioned that the RHs follow a correlation in the $\mathrm{P}_{1.4}-\mathrm{L}_{X}$ plane for a definite time-scale and in later stages of the merging, radio halos enter a fading phase and the location of their parent clusters gradually move towards the radio quiet region of the plane. They pointed out that their speculation is in line with the observational predictions in the primary electron model $(\S 2.7)$. In the re-acceleration model, merging has an effective influence in re-accelerating the particles of the ICM and the life-time of radio halo is heavily dependent on the strength of the merger-driven turbulent.

Deep radio and X-ray observations of the REXCESS (see $\S 4.2$ ) clusters with declinations below $-20^{\circ}$ have provided an ideal laboratory to probe the properties of clusters with and without halos. The X-ray luminosity and some global properties of our observed REXCESS sub-sample are given in Table 7.1. Following the bi-modality investigations in past RH surveys, we have made use of an updated integrated radial brightness profile derived by Pratley (2012) to generate a number of fake radio halos to estimate the upper-limit flux in our non-detection clusters. As mentioned in $\S 4.1$, we have observed a morphologically unbiased sample of galaxy clusters with a homogeneous X-ray luminosity selection with the aim of discovering more radio halos and investigating the 
proposed correlations and bi-modality $(\S 4.1)$ with data that suffers minimum selection effects.

In this chapter, we present the results of updated radio/X-ray, radio/SZ and radio/mass scaling relations and discuss the selection effects in those correlations. We also present the result of derived upper-limits for clusters with non-detected halos and explain the procedure of injecting fake radio halos into the uv-data and compare the results with other radio halo surveys. We show our non-halo clusters alongside the other non-detections from the literature on the $\mathrm{P}_{1.4}-\mathrm{L}_{X}, \mathrm{P}_{1.4}-\mathrm{Y}_{500}$ and $\mathrm{P}_{1.4}-M_{500}$ planes to study the matter of bi-modality in our observed clusters. We consider the two most disturbed clusters with no sign of cluster scale diffuse radio emission in our observed sub-sample and discuss the possibilities to address the issue of non-detection in these two clusters.

In this chapter we assume a $\Lambda$ CDM cosmology with $\mathrm{H}_{0}=70 \mathrm{kms}^{-1} \mathrm{Mpc}^{-1}, \Omega_{m}=0.3$ and $\Omega_{\Lambda}=0.7$.

\section{§7.2. Fake Radio Halo Injection}

It is thought that the radio halo brightness profile carries invaluable information about the distribution of the ICM and magnetic field in clusters. Thus, Cassano et al. (2007) carefully scrutinised five GRHs with high quality available data from the literature to determine the integrated radial brightness profile of radio halos for the first time. They determined the integrated radio brightness profile of GRHs in clusters A2319 (Feretti et al., 1997), A2255 (Govoni et al., 2005), A2163 (Feretti et al., 2001), A2744 (Govoni et al., 2001) and A545 (Bacchi et al., 2003) and then derived the combined normalised integrated brightness profile of the aforementioned radio halos. They found that the integrated brightness profile of different radio halos are in quite good agreement with each other whilst the halo-centric radius is normalised to the size of the radio halo. Soon after, Brunetti et al. (2007) and Venturi et al. (2008) used this radial brightness profile to inject some Gaussian halos with a fixed radius of $500 \mathrm{kpc}$ and various fluxes into the uv-data of the observed clusters in the GRHS with no sign of diffuse radio emission and then they imaged the modified uv-data. They reported that in all cases, the total flux and largest linear size (LLS) were partially recovered. They pointed out that the central part of the halos was well detected, however, most of the emission loss occur at the outermost regions of the halos as the brightness decreases. They reported that a maximum of 50\% of the flux and $30 \%$ of the initial LLS were lost in the radio images with the fake-halos. They found that fake halos with a flux range of 5-12 mJy made reasonable extended diffuse emission at 4-5 times the rms noise of the images. However, there is a caveat in that they have not explicitly defined a firm constraint on the LLS of the detection. In 
other words, they have not defined a radio halo detection threshold with respect to the recovered total flux or measured LLS. Years later, Kale et al. $(2013,2015)$ performed the same procedure to constraint the upper limits of the halo non-detections in the EGRHS sample. They injected synthesised $1 \mathrm{Mpc}$ diameter circular fake radio halos in the nonhalo clusters. They reported that the flux of the detection limit differed from 2 to $7 \mathrm{mJy}$ at $610 \mathrm{MHz}$. Kale et al. (2013) also have included another well-known correlation in their radio halo injection procedure. In particular, they considered the scaling relation between the size of radio halos and the X-ray luminosity of the clusters. It is known that the more extended radio halos have been observed in the clusters with the highest X-ray luminosities (Cassano et al., 2007). Kale et al. (2013) pointed out that merely three clusters had a different halo extent than predicted by this correlation. Further more, once they injected a halo with the predicted LLS (according to the X-ray luminosity of the cluster), they did not observe a noticeable change in the upper limit halo flux.

Pratley (2012) employed an identical approach and studied the same five well-known radio halo profiles to derive the normalised integrated brightness profile of radio halos. Additionally, in an extension to the previous work, he also applied a polynomial fit to the profile to be able to provide a general model of the brightness profile to inject a fake elliptical halo into the radio data-set of the cluster A3667. Adapting an elliptical shape allowed more flexible radio halos which could better follow the morphology of the X-ray emission of the ICM. Pratley (2012) reported that the best polynomial fit to the normalised integrated brightness profile was $\mathrm{I}(r)=-0.719 r^{2}+1.876 r-0.141$.

We have injected a series of elliptical radio halos with the LLS of $1 \mathrm{Mpc}$ using the polynomial fit proposed by Pratley (2012) to obtain the brightness for each pixel of the injected halo in our radio maps. Based on the interferometry principles, the total flux is:

$$
S=\int B(r) d A
$$

where $\mathrm{B}(\mathrm{r})$ is the radial brightness and $\mathrm{dA}$ is the area element. If we consider the general equation form of an ellipse :

$$
\frac{\left[\left(x-x_{0}\right) \cos \beta+\left(y-y_{0}\right) \sin \beta\right]^{2}}{A^{2}}+\frac{\left[\left(y-y_{0}\right) \cos \beta-\left(x-x_{0}\right) \sin \beta\right]^{2}}{B^{2}}=r^{2} .
$$

then the region enclosed within an ellipse is defined when $r \leq 1$. Parameters $x_{0}$ and $y_{0}$ were the centre of the ellipse and $\beta$ was the rotation angle. Parameters A and B are semi major and minor axes of the ellipse.

Pratley (2012) mentioned that the $\mathrm{x}$ and $\mathrm{y}$ in this equation can simply be interpreted as the pixels of the image and $r$ is the radial distance from each pixel to the centre of the ellipse. Since in this new coordinate system, $\mathrm{r}$ is always less than 1 , then it could be treated as a normalised radial distance and can be used to derive the normalised 

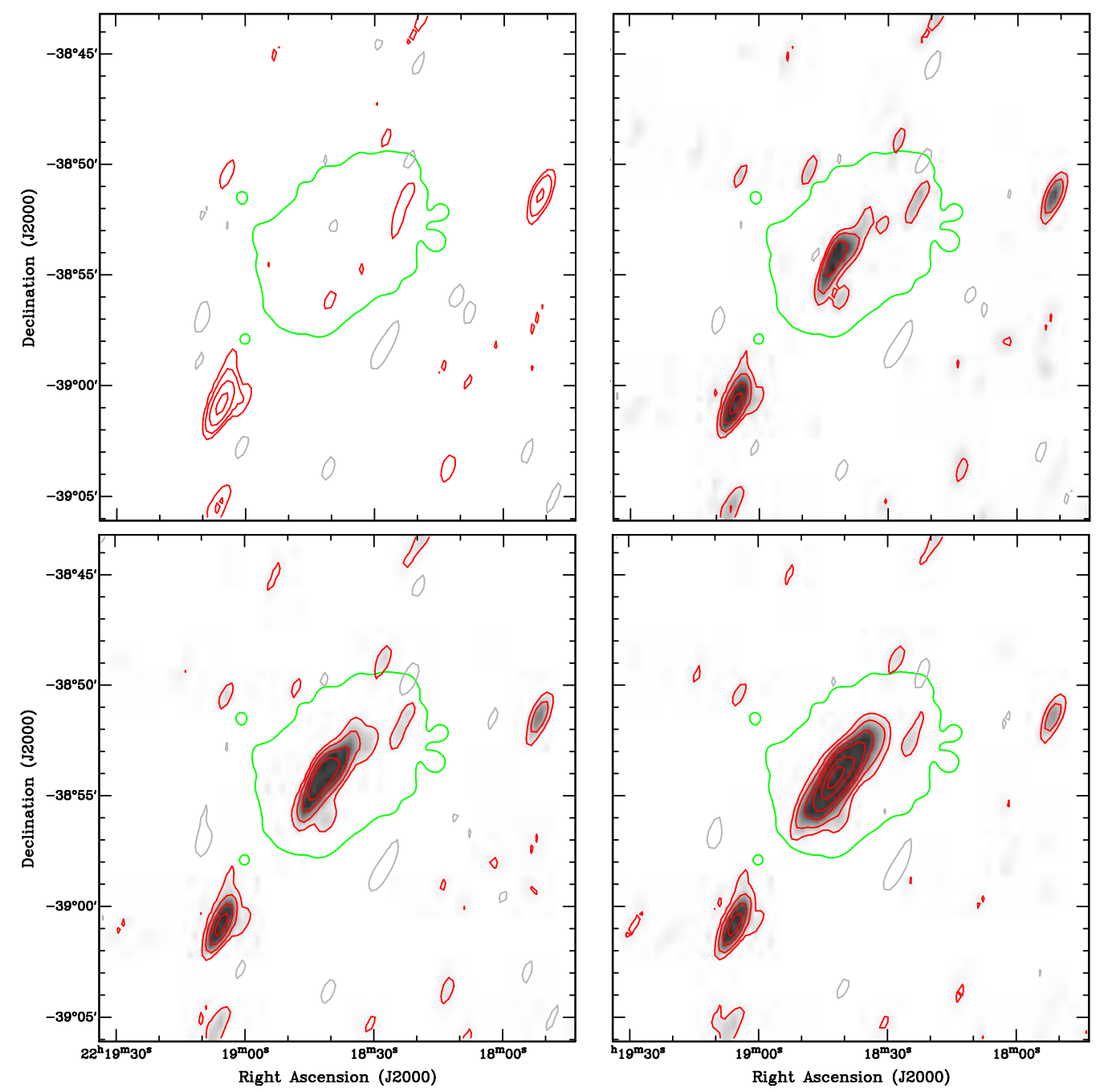

Figure 7.1: Example of a injected halo in the cluster RXCJ2218.6-385: The images of injected fake RH with total flux of $0,12,25,50 \mathrm{mJy}$, respectively are shown, from top left to right bottom. Red contours represent the radio data at $1.4 \mathrm{GHz}$ and green contour represents the outer contour of the XMM-Newton X-ray data. The red contours start at $0.7 \mathrm{mJy}_{\text {beam }}^{-1}$ and increase by factor of 2 . The grey contour shows the negative value of $-0.7 \mathrm{mJy}$ beam $^{-1}$.

integrated brightness profile. The integrated brightness can be written as:

$$
I(r)=2 \pi \times A B \int_{0}^{1} B(r) r d r .
$$

In the above equation, $A=1$ and $B<1$. If we re-write the above equation in our new coordinate system, the brightness of each pixel can be calculated as:

$$
S_{t} \times \frac{d I}{d r} \times \frac{B^{\prime}}{2 \pi A^{\prime}} \times \frac{1}{r}
$$

Where $\mathrm{A}^{\prime}$ and $\mathrm{B}^{\prime}$ are now in pixels and $S_{t}$ is the total integrated flux.

We used the task "uvmodel" in the MIRIAD software (Sault et al., 1995) to inject the modelled radio halo into the uv-components of our dataset and then the standard 
imaging and CLEANing were performed. Here we mention some comments about the modified uv-data once the synthesised uv-components were injected into the data set. As Pratley (2012) mentioned, it is virtually impossible to completely recover the total flux of the injected fake halo, due to the incomplete sampling of the uv-data by an interferometer. Thus, Fourier components are lost during the imaging process. This means that the total flux of the injected halo is always an upper limit of the total measured halo flux in the image. On the other hand, it should be noted that once the fake visibilities are injected to the data, the uv-coverage is not identical to the non-modified uv-data. This is also true when the visibilities of the point sources are subtracted from the uv-data.

Pratley (2012) reported that an elliptical radio halo with LLS of $1 \mathrm{Mpc}$ was injected to the uv-data of the cluster A3667 and he used a detection threshold where the detection was made when at least $25 \%$ of the initial surface was recovered at $3 \sigma$ detection. $\mathrm{He}$ reported that the upper limit of the detection was $75 \mathrm{mJy}$ whilst the local rms noise level of the radio map was $1.3 \mathrm{mJy}^{\text {beam }^{-1}}$ and $25.1 \%$ of the surface was detected.

In our work, the centre of the synthesised elliptical halos were placed on the $\mathrm{X}$ ray peak position. In all the non-halo clusters, we have peeled all the radio sources in the central region of the X-ray emitting region to be able to measure the precise halo extent in each cluster. The only cluster with no radio source subtraction was the cluster RXCJ0049.4-2931 in which there was no radio source in the X-ray region of the cluster. We noticed that there were neither a strong residual nor the subtraction artefacts in the peeled radio images and the local rms noise remained roughly constant. We used the same detection threshold as employed by Pratley (2012). One example of an injected fake halo into the cluster RXCJ2218.6-385 with different fluxes is shown in Figure 7.1.

Here we mention some points about our halo injection procedure.

- The largest spatial scale detectable in our uv-data is $24^{\prime}$. However, a radio halo with $1 \mathrm{Mpc}$ diameter would subtend $15.3^{\prime}$ in the cluster with the lowest redshift 0.0564 at $1.4 \mathrm{GHz}$. This has assured us that a low-surface brightness radio halo with $1 \mathrm{Mpc}$ extend is detectable in all of our clusters.

- Cluster RXCJ0049.4-2931 had the highest upper limit of 24 mJy when about 25\% of the surface area had pixel values over $3 \sigma$. This might be due to the higher rms noise and consequently, lower sensitivity level of the tapered image. However, we note that this cluster had the highest noise level in the original tapered images.

- We note that according to radio halo statistics, clusters with higher X-ray luminosity host more extended halos. This is, in particular, helpful to detect halos in clusters with lower X-ray luminosity. Thus, we should have injected smaller halos in clusters with lower X-ray luminosity and accordingly, our upper limits 
Table 7.2: Upper limits of the flux and power derived in the halo injection procedure in the non-halo clusters.

\begin{tabular}{lcl}
\hline \hline $\begin{array}{c}\text { Cluster } \\
\text { Name }\end{array}$ & $\begin{array}{c}\mathrm{S}_{1.4} \\
\mathrm{mJy}\end{array}$ & $\begin{array}{l}\mathrm{P}_{1.4} \times 10^{23} \\
\mathrm{~W} \mathrm{~Hz}^{-1}\end{array}$ \\
\hline RXCJ0006.0-3443 & $<7$ & $<2.46$ \\
RXCJ0049.4-2931 & $<24$ & $<7.45$ \\
RXCJ0145.0-5300 & $<12.5$ & $<4.57$ \\
RXCJ0211.4-4017 & $<6$ & $<1.60$ \\
$R X C J 0225.1-2928$ & $<11$ & $<9.80$ \\
$R X C J 0345.7-4112$ & $<13$ & $<1.33$ \\
$R X C J 0616.8-4748$ & $<5.5$ & $<1.99$ \\
$R X C J 0645.4-5413$ & $<6.5$ & $<5.06$ \\
$R X C J 1236.7-3354$ & $<11$ & $<1.76$ \\
$R X C J 2014.8-2430$ & $<5.5$ & $<4.10$ \\
$R X C J 2023.0-2056$ & $<20$ & $<1.54$ \\
$R X C J 2129.8-5048$ & $<16$ & $<2.55$ \\
$R X C J 2149.1-3041$ & $<7.5$ & $<2.82$ \\
$R X C J 2218.6-3853$ & $<7$ & $<3.87$ \\
& & \\
\hline
\end{tabular}

could have been deeper. However, for the sake of comparison with the GRHS and EGRHS, we chose to inject radio halos with an identical extent regardless of the X-ray luminosity of clusters.

The details of the upper limit flux and the power at 1.4 GHz are given in Table 7.2. All the power limits were calculated based on a halo spectral index 1.3.

In the following sections, the radio halo fraction in our sample and the observed bi-modality in all the radio halo scaling relations are discussed.

\section{§7.3. Radio Halo Fraction}

Determining the fraction of radio halo occurrence in galaxy clusters is one of the major goals in radio halo studies. Cassano et al. (2008) reported that the halo fraction was 29 $\pm 9 \%$ for the entire sample in the GRHS. Moreover, they investigated the halo fraction as a function of X-ray luminosity and used an $\mathrm{L}_{X}$ threshold of $8 \times 10^{44} \mathrm{erg} \mathrm{s}^{-1}$ and found $\mathrm{f}_{R H}$ to be $40.6 \%$ and $7.5 \%$ for clusters with $\mathrm{L}_{X} \geq 8 \times 10^{44} \mathrm{erg} \mathrm{s}^{-1}$ and $5 \times 10^{44} \leq \mathrm{L}_{X} \leq$ $8 \times 10^{44}$ erg s$^{-1}$, respectively.

As mentioned earlier, 64 galaxy clusters were observed in the GRHS+EGRHS to search for radio halos, however, only 58 clusters had reliable radio data. Kale et al. (2015) reported that in the full GRHS+EGRHS sample, 13 clusters had radio halos and pointed out that $\mathrm{f}_{R H}$ is (13/58) 22\% in the combined sample. Kale et al. (2013) 
Table 7.3: Radio halo fraction in different X-ray luminosity bins, in disturbed clusters and in full cluster sample are given.

\begin{tabular}{llllll}
\hline \hline & $\mathrm{L}_{X}{ }^{\mathrm{a}} \leq 5$ & $5 \leq \mathrm{L}_{X}^{\mathrm{a}} \leq 8$ & $8 \leq \mathrm{L}_{X}^{\mathrm{a}}$ & $\begin{array}{l}\text { Disturbed } \\
\text { Clusters }\end{array}$ & $\begin{array}{l}\text { Total } \\
\text { Sample }\end{array}$ \\
\hline GRHS & N/A & $7 \%$ & $41 \%$ & $73 \%$ & $29 \%$ \\
EGRH+GRHS & 0 & $14 \%$ & $28 \%$ & $43 \%$ & $22 \%$ \\
GBT & $30 \%$ & $100 \%$ & $100 \%$ & $41 \%$ & $41 \%$ \\
This work & 0 & $50 \%$ & 0 & $11 \%$ & $7 \%$ \\
\hline
\end{tabular}

${ }^{\mathrm{a}} \mathrm{X}$-ray luminosities in units $10^{44} \mathrm{erg} / \mathrm{s}$.

found that $\mathrm{f}_{R H}=31 \%$ and $11 \%$ in X-ray luminosity bins of $\mathrm{L}_{X} \geq 8 \times 10^{44} \mathrm{erg} \mathrm{s}^{-1}$ and $5 \times 10^{44} \leq \mathrm{L}_{X} \leq 8 \times 10^{44} \mathrm{erg} \mathrm{s}^{-1}$, respectively. They mentioned that their results are consistent with the reported fractions in the GRHS. However, in a recent work (Kale et al., 2015) they found no statistical difference in halo fraction in the lower/higher Xray luminosity bins. We also have estimated the halo fraction in the work presented by Farnsworth et al. (2013) to be $\mathrm{f}_{R H}=33 \%$ including all the clusters in their sample. The halo fraction was $9 \%$ in clusters with $\mathrm{L}_{x}>8 \times 10^{44} \mathrm{erg} \mathrm{s}^{-1}, 9 \%$ in clusters with $5 \times$ $10^{44}<L_{x}<8 \times 10^{44} \mathrm{erg} \mathrm{s}^{-1}$ and $\mathrm{f}_{R H}=17 \%$ for clusters with $\mathrm{L}_{x}<5 \times 10^{44} \mathrm{erg} \mathrm{s}^{-1}$.

We note that except for the cluster RXCJ0049.4-2931 with a maximum rms noise of $313 \mu \mathrm{Jy}_{\text {beam }}^{-1}$, the rms noise of the remaining low resolution images in our subsample varies from $116-214 \mu \mathrm{Jy}$ beam $^{-1}$ with the average rms noise of $157 \mu \mathrm{Jy}$ beam $^{-1}$. The noise level of the tapered images in the GRHS project varies between 35 to $100 \mu \mathrm{Jy} \mathrm{beam}^{-1}$ and $\sigma_{r m s}$ was $40-85 \mu \mathrm{Jy}_{\text {beam }}{ }^{-1}$ in the EGRHS project. Hence, the average rms noise of our images are about 2-3 times greater than the average noise level of the tapered images in the GRHS and EGRHS. Additionally, since halos have steep spectrum, it is more likely to detect a halo at $610 \mathrm{MHz}$ than to $1.4 \mathrm{GHz}$ as halos have more flux and might be even more extended at lower frequencies.

The halo fraction in our sample was $\mathrm{f}_{R H}=(1 / 15)=7 \%$. The detection of only one radio halo in our 15 observed galaxy clusters signifies that radio halos are very rare and hard to find. The halo fraction was $50 \%$ and 0 in X-ray luminosity bins $5 \times 10^{44} \leq \mathrm{L}_{X} \leq$ $8 \times 10^{44} \mathrm{erg} \mathrm{s}^{-1}$ and $\mathrm{L}_{X} \geq 8 \times 10^{44} \mathrm{erg} \mathrm{s}^{-1}$, respectively. Details of the halo fraction in different radio halo surveys is given in Table 7.3.

Cassano et al. (2010) have also investigated the halo fraction as function of parent cluster's morphology. They pointed out that $73 \%$ of the merging clusters in the redshift range 0.2 to 0.32 in the GRHS had radio halos. According to the reported dynamical status of the clusters in our sub-sample, $60 \%$ of the clusters are disturbed clusters and the remaining clusters are relaxed. Given that the detected radio halo in our sub-sample is present in an intermediately disturbed cluster, the halo fraction in our disturbed clus- 
ters is $11 \%$. We note that the majority of reported relaxed clusters only have X-ray data available without any optical analysis available in the literature. Hence, the dynamical information of such relaxed clusters should be re-examined at the time the optical substructure analysis become available in the future which opens the possibility that the reported halo fraction as function of morphology could change if significant substructure along the LOS is found.

\section{§7.4. Radio / X-ray Luminosity: Scaling Relation and Bi- modality}

In multiple radio halo studies the scaling relations between the thermal and non-thermal components of halo clusters have been investigated to infer possible connections. The first connection was observed by Burns et al. (1995) and Colless and Dunn (1996) where they noticed a link between the dynamical state of the parent cluster and the occurrence of radio halos. Later on, Liang et al. (2000) observed a tight correlation between the $\mathrm{X}$-ray luminosity measured in $0.1-2.4 \mathrm{keV}$ range and the power of the halo measured at $1.4 \mathrm{GHz}$. This finding was later confirmed by Cassano et al. (2006); Brunetti et al. (2009) and Cassano et al. (2011) when more quality radio data became available.

In conjunction with the designed radio halo surveys, Brown et al. (2011) used the available SUMSS survey images to conduct a radio halo survey using image stacking technique to overcome the known sensitivity issue in the SUMSS survey in finding lowsurface brightness objects. They performed a comprehensive statistical study of stacked images of 105 massive galaxy clusters in the SUMSS survey. They found that there is a population of "off-state" clusters with giant radio emissions where their radio power is roughly an order of magnitude less than what is expected from the current X-ray/radio power scaling relation ("on-state" halos). They reported that the observed "off-state" emission increases in the X-ray luminous and dynamically disturbed clusters. They also proposed that this type of giant radio halos can be generated via the secondary electrons in the clusters. They claimed that the radio emission from the secondary electrons in "off-state" and non-turbulent systems are roughly 10 times less powerful than the radio halos generated by turbulence. ${ }^{1}$ According to Figure 7.2, the location of our halo in A3888 is well above the "off-state" limit.

In a recent work, Cassano et al. (2013) gathered the information on 25 well-studied radio halos with available measured errors both in the X-ray luminosity and halo power at $1.4 \mathrm{GHz}$. They applied the Bivariate Correlated Errors and intrinsic Scatter (BCES)

\footnotetext{
${ }^{1}$ There is only one radio halo directly found in the "off-state" region by Bonafede et al. (2015) in the cluster CIZA J1938.3+5409 with the GMRT. However, more follow-up observations in higher frequency such as $1.4 \mathrm{GHz}$ might be useful for further confirmation of this diffuse source.
} 
linear regression method (Akritas and Bershady, 1996) to the data and determined the most precise correlation between the X-ray luminosity of the parent cluster and the radio halo power at $1.4 \mathrm{GHz}$. Unfortunately, an extra 36 halos from the literature were excluded in the Cassano et al. (2013) work due to not having available X-ray luminosity errors in the catalogues. Thus, many of the halos, in particular halo clusters with low X-ray luminosity were excluded from the fit presented by Cassano et al. (2013).

To extend this investigation by including more recent discoveries, we have gathered information from extra 37 radio halos ( 36 from the literature and one from this work) to verify whether the previous established correlation is still valid if lower-mass clusters with $\mathrm{L}_{X} \leq 10^{44.5} \mathrm{erg} / \mathrm{s}\left(\approx 3 \times 10^{44}\right)$ are included in the fitting procedure. It should be noted that our fit to all 62 radio halos (see Appendix B.1) was a simple polynomial fit and due to the lack of X-ray luminosity errors in the publications, we were not able to perform a BCES fit to all data. Figure 7.2 shows the correlations derived by Cassano et al. (2013) (black dashed line, fit to blue dots enclosed by black circles) and the correlation derived in this work (red dashed line, fit to all 62 blue dots). The red point is the halo presented in this work. The stacked results from Brown et al. (2011) are marked with green pluses and the green dashed line represents the upper limit of "off-state" (Brown et al., 2011) in this plot. According to Figure 7.2, it is very clear that the black fit by Cassano et al. (2013) lacks information of the X-ray under-luminous halo clusters. However, according to Figure 7.2 , there are merely $10(\approx 16 \%$ of all data points) radio halos that have an X-ray luminosity less than $10^{44.5} \mathrm{erg} / \mathrm{s}$ and exhibit more scatter with respect to rest of the radio halos. The location of $\mathrm{A} 3888$ on the $\mathrm{P}_{1.4}-\mathrm{L}_{X}$ plane is very close to the black fit and accordingly has a power that is consistent with the Cassano et al. (2013) fit. Interestingly, our halo in A3888 is also in agreement with the fit (red dashed line) derived in this work.

As mentioned in $\S 7.2$, we have injected a series of elliptical fake halos into the non-halo clusters in our REXCESS sub-sample to obtain firm upper limits of the detections. Beside the upper limits presented in the GRHS and EGRHS (see black arrows in Figure 7.2), other upper limits have been reported in more recent papers, for instance, Russell et al. (2011) carried out a radio observation of Abell 2146 (A2146) with the GMRT at $325 \mathrm{MHz}$ and found no cluster-scale emission in this merging cluster. They injected a $1 \mathrm{Mpc}$ diameter radio halo into the radio data and derived the upper limit of the flux of the non-detected halo in this cluster. We have marked the derived upper-limit power of A2146 with a blue arrow in Figure 7.2. Later on, Farnsworth et al. (2013) studied 12 merging clusters with redshift less than 0.2 and in a wide X-ray luminosity range between $10^{43}-10^{45} \mathrm{erg} \mathrm{s}^{-1}$ with the Green Bank Telescope (GBT) at $1.4 \mathrm{GHz}$. They found two new radio halos (A2065 and A2069) and more excess diffuse emission from previously known halos in A2061 (van Weeren et al., 2011a) and A2319 (Harris 


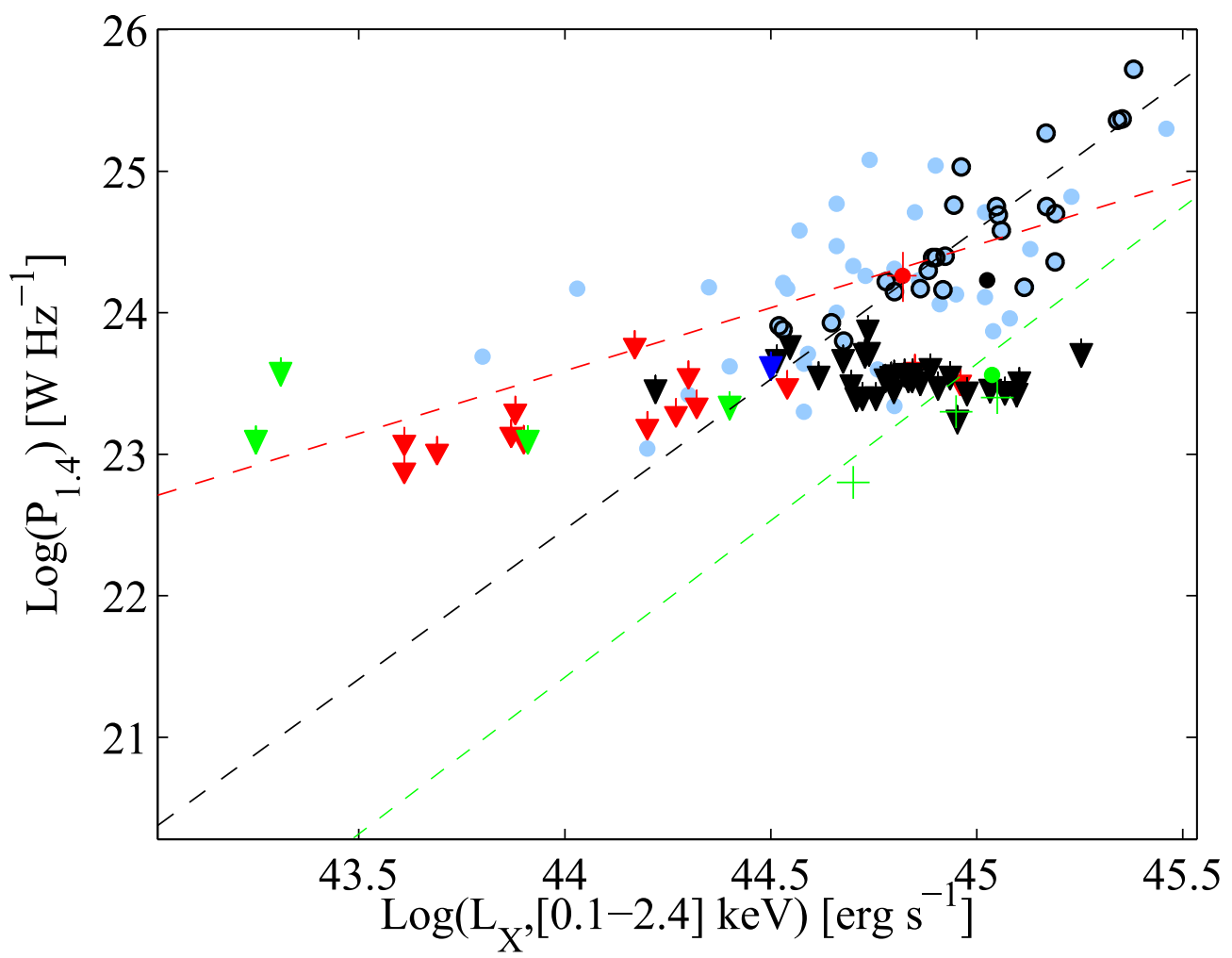

Figure 7.2: Distribution of the halo and non-halo clusters on the $\mathrm{P}_{1.4}-\mathrm{L}_{X}$ plane. Blue dots represent all the radio halos available in the literature and blue dots with black circles around them indicate the halos included in the Cassano et al. (2013) fit. The red dot indicates the radio halo in the A3888. The upper limits of our non-halo clusters are shown with red arrows. Black arrows show the upper limits of the GRHS+ EGRHS and the green arrows represent the upper limits of the GBT project (see Farnsworth et al., 2013 and references therein). The blue arrow shows the upper limit of A2146 (Russell et al., 2011). The black dashed line is the fit derived by Cassano et al. (2013) and red dashed line is the simple linear fit to all 62 radio halos derived in this work. The green pluses represent the halos found in the stacked radio images in (Brown et al., 2011) and dashed green line indicates the approximate "off-state" upper limit. The green dot shows the first direct halo detection in the "off-state" region (Bonafede et al., 2015). For the sake of clarity we only have shown the error bars of the halo in A3888. The total sample of detected and upper limit halos used here are 62 and 49, respectively. the errors of the power and $\mathrm{L}_{X}$ of all the radio halos are reported in Appendix B.1.

and Miley, 1978). We have marked the upper limits derived by Farnsworth et al. (2013) with green arrows in Figure 7.2. The upper limits derived in this work are indicated with red arrows in this plot.

According to the locations of our upper limits on the $\mathrm{P}_{1.4}-\mathrm{L}_{X}$ plot only three clusters with $\mathrm{L}_{X}>10^{44.5}$ have measured power limits less than the halo power predicted by Cassano et al. (2013). Thus, the majority of our upper limits are very close or higher than the predicted halo power particularly for the clusters with the X-ray luminosity less than $10^{44.2} \mathrm{erg} \mathrm{s}^{-1}$. However, if we consider the fit derived in this work which has taken all the radio halos into account (red dashed line) all of our derived upper limits (except 
the two which are very close to our fit) settle well under the predicted halo power level from our fit.

According to the Cassano et al. (2013) fit, the majority of radio observations in this work were not sufficiently sensitive to detect radio halos with the predicted halo power. However, if we consider the correlation derived from all known halos, they are sufficiently sensitive to detect halos. According to the Cassano et al. (2013) fit, more sensitive radio observations are required to either detect radio halos with such low power predicted by the correlation or to obtain deeper upper limits. For the clusters with upper limits close to the predicted halo power, low-frequency observation with the MWA-II will be useful to verify the presence of any USS halos which may be yet undetected. Additionally, if we consider the correlation derived from all detected halos, the bi-modality is also not robust as some of the upper limits and halos are mixed in the $\mathrm{P}_{1.4}-\mathrm{L}_{X}$ plane.

As mentioned before, the assumption that halos in less massive clusters are still at Mpc scales is at odds with the halo LLS/ $\mathrm{L}_{X}$ correlation. Thus, we also suggest that the derived upper limits in this work could be improved by injecting fake halos with a radius which is determined based on the current LLS/ $\mathrm{L}_{X}$ correlation (see Figure 7.5). This treatment might be useful to obtain deeper upper limits in our X-ray under-luminous clusters and accordingly, might suggest that our radio observations were sufficiently sensitive to detect possible halos. Additionally, this approach might provide an opportunity to better investigate the bi-modality, but would require older upper limits for halos in the literature to be re-calculated accordingly.

In the next section the bi-modality in the $\mathrm{P}_{1.4}-\mathrm{Y}_{S Z}$ plane is discussed.

\section{§7.5. Radio / SZ and Radio / Mass Relations and Bi-modality}

Cosmic Microwave Background (CMB) photons experience the Inverse Compton (IC) effect when encountering ultra-relativistic electrons in the ICM. In this process, lowenergy CMB photons gain energy via interaction with higher energy electrons are blueshifted and the spectrum of the CMB photons is distorted. This phenomenon is known as the Sunyaev-Zeldovich (SZ) effect. Although only a very small fraction (1\%) of the CMB photons experience IC scattering during the passage of the ICM, it is sufficient to distort the CMB spectrum. This can be traced by identifying a fall and a rise occurring respectively in the $\mathrm{CMB}$ intensity at frequencies close to $218 \mathrm{GHz}$. This imprint can be used as a powerful tool to find galaxy clusters. Sunyaev and Zeldovich $(1970,1972)$ defined the intensity of the SZ signal (also known as Compton parameter) to be $y=$ $\int n_{e} T_{e} d l$ in an arbitrary direction, where $\mathrm{n}_{e}$ is the electron number density and $\mathrm{T}_{e}$ is the electron temperature and the integral is calculated along the LOS. When the y-parameter 
is integrated over the subtended solid angle $(\Omega)$ by the cluster, the integrated Compton parameter is derived and is called the SZ flux.

$$
\text { SZ flux } \propto Y=\int y d \Omega \propto \frac{1}{D_{A}^{2}} \int_{0}^{\infty} d l \int_{A} n_{e} T_{e} d A
$$

where $\mathrm{D}_{A}$ is the angular diameter distance and $\mathrm{A}$ is approximately the cluster area when it is projected on the plane of sky. As it is shown in the above equation, the SZ intensity is independent of redshift.

Recent SZ observations of the clusters with the Planck satellite, the South Pole Telescope (SPT) and the Atacama Cosmology Telescope (ACT) have discovered many unknown galaxy clusters at high redshifts and also have provided invaluable information about the ICM of the clusters. The Planck measured integrated SZ signal for A3888 in an $5 \mathrm{R}_{500}{ }^{2}$ aperture was $0.00566 \pm 0.0007198\left[\operatorname{arcmin}^{2}\right]$. The definition of the SZ flux in the literature might be different in terms of using different aperture and different units. One can convert the SZ flux unit from $\left[\operatorname{arcmin}^{2}\right]$ to $\left[\mathrm{Mpc}^{2}\right]$ using the conversion given by:

$$
Y_{S Z}\left[\mathrm{Mpc}^{2}\right]=8.46 \times 10^{-8} Y_{S Z}\left[\operatorname{arcmin}^{2}\right]\left(\frac{D_{A}}{1 \mathrm{Mpc}}\right)^{2} .
$$

If we use the above conversion, we obtain the $\mathrm{Y}_{S Z}$ to be $1.56 \times 10^{-4}\left[\mathrm{Mpc}^{2}\right]$ for $\mathrm{A} 3888$ in an $5 \mathrm{R}_{500}$ aperture. In some publications the $\mathrm{SZ}$ flux is defined as $\mathrm{Y}_{500}$, however, $\mathrm{Y}_{5 R_{500}}$ is often reported as the measured integrated Compton parameter. It should be noted that the equivalent SZ flux measured in an $5 \mathrm{R}_{500}$ aperture is $1.79 \times \mathrm{Y}_{500}$ (Arnaud et al., 2010). If we use the mentioned conversion, the SZ flux for A3888 is $8.7 \times 10^{-5}$ $\mathrm{Mpc}^{2}$ in an $\mathrm{R}_{500}$ aperture which is close to the measured $\mathrm{Y}_{500}$ value of $8.82 \times 10^{-5}$ $\mathrm{Mpc}^{2}$ by Arnaud et al. (2010).

The first radio/SZ correlation for the halo clusters was proposed by Basu (2012a). They used the Planck Early Sunyaev-Zel'dovich (ESZ) catalogue (Planck Collaboration et al., 2011) to scrutinise the radio/SZ correlation for the known radio halos in the literature. They observed a correlation between the power of the halos and the observed SZ signal of the halo clusters in the literature. Soon after, Cassano et al. (2013) made use of the Planck Sunyaez-Zeldovich Sources Release 1 (PSZ1) $)^{3}$ catalogue (Planck Collaboration et al., 2013) to find $Y_{S Z}$ measurements for 25 radio halos that were included in their $\mathrm{X}$-ray/radio $\left(\mathrm{P}_{1.4}-\mathrm{L}_{X}\right.$ plane) correlation calculation. They then applied the BCES linear regression to find the slope of the correlation. With the aim of further investigation and including more SZ measurements for radio halos, we have collected Planck $\mathrm{Y}_{S Z}$ measurements for an extra 28 radio halos from the literature in the Planck catalogue of

\footnotetext{
${ }^{2} R_{500}$ is defined as the radius of a sphere where the average density is 500 times the current critical density of the Universe.

${ }^{3} \mathrm{PSZ} 1$ catalogue is catalogue of galaxy clusters detected in the Planck all-sky survey during the first 15.5 months of mission.
} 
Table 7.4: Slope of the best-fit of scaling relations derived by Cassano et al. (2013) and this work.

\begin{tabular}{lccc}
\hline \hline Scaling relation & $\mathrm{P}_{1.4}-\mathrm{L}_{500}$ & $\mathrm{P}_{1.4}-\mathrm{y}_{S Z}$ & $\mathrm{P}_{1.4}-\mathrm{M}_{500}$ \\
\hline $\begin{array}{c}\text { Cassano et al. (2013) } \\
\text { This work }\end{array}$ & 2.11 & 2.03 & 3.70 \\
& $0.89^{\mathrm{a}}$ & 2.29 & 3.76 \\
\hline $\begin{array}{l}\text { a Simple linear polynomial fit. The BCES fit was not possible due to the } \\
\text { lack of reported } \mathrm{L}_{X} \text { errors. }\end{array}$
\end{tabular}

Sunyaez-Zeldovich Sources Release 2 (PSZ2) ${ }^{4}$ catalogue (Planck Collaboration et al., 2015 ) to derive a more precise correlation between the halo power and the SZ signal of halo clusters.

We found that the slope of our BCES Bisector linear regression fit to these 53 data points to be 2.29 while Cassano et al. (2013) reported the slope of their fit was 2.03. Since the Planck has only found massive clusters, the remaining nine clusters in the literature were not included in our fitting procedure. The missing clusters are the ones with $\mathrm{L}_{X}<10^{44.5} \mathrm{erg} / \mathrm{s}\left(\approx 3 \times 10^{44}\right)$. Given that the low mass systems are not included in our fitting our correlation merely represents large mass systems and is prone to a significant change once the $\mathrm{Y}_{S Z}$ measurements of the remaining radio halos (with no current SZ measurements) become available and are taken into account. Thus, it is not surprising that the reported slope for the fit derived by Cassano et al. (2013) is not dramatically different from our fit. We plot our radio halo along the other radio halos from the literature in the $\mathrm{P}_{1.4}-\mathrm{Y}_{S Z}$ plane in Figure 7.3. The location of A3888 is in line with both the correlations (black and red dashed lines).

Cassano et al. (2013) also have investigated the connection between the radio halo power and the mass of the parent cluster. They found a linear correlation in the $\mathrm{P}_{1.4}$ $M_{500}$ plane for the same 25 radio halos used previously in the other correlations. We searched the PSZ2 catalogue and found an extra 28 (including A3888) $\mathrm{M}_{500}$ measurements to derive a more precise fit to the data. We then applied the BCES Bisector fitting to all the 53 radio halos and found the slope to be 3.76. Table 7.4 gives the information about our fits and the fits derived by Cassano et al. (2013). Similar to the $\mathrm{P}_{1.4}-Y_{S Z}$ plane, there was no information available for the remaining low-mass systems in the literature. Thus, the correlation is only valid for massive halo clusters and once the mass of the lower mass systems becomes available, a more unbiased correlation can be obtained. In bottom panel of Figure 7.3 we have marked our halo alongside the halos in the literature on the $\mathrm{P}_{1.4}-M_{500}$ plane with a red filled dot. The location of our halo is in agreement with the predicted value from the fits derived in this work and by Cassano et al. (2013).

\footnotetext{
${ }^{4}$ PSZ2 catalogue is catalogue of galaxy clusters detected in the Planck all-sky survey during the fullmission (29 months).
} 
As mentioned earlier Basu (2012a) observed a correlation between the power of radio halos and the integrated SZ signal of the parent clusters. However, they claimed that they found no evidence of a distinct bimodal behaviour as observed in the radio/X-ray distribution. Soon after, Cassano et al. (2013) cross-correlated the non-halo clusters in both the EGRHs + GRHS with the PSZ1 catalogue and found the SZ measurements for 19 out of 29 non-halo clusters. In order to use the remaining 10 non-halo clusters, they studied the correlation of the $\mathrm{Y}_{500}$ (see 7.5) and the X-ray luminosity in the [0.15$1] R_{500}$ region to determine the estimated $Y_{500}$ values. For the sake of this experiment, they scrutinised 63 clusters with the best quality X-ray and SZ dataset available in the XMM-Newton and Planck archives, respectively. They also verified the accuracy of the predicted $\mathrm{Y}_{500}$ from the X-ray observations by comparing the actual PSZ1 SZ measurements with the predicted $Y_{500}$ values and found that both the estimated and measured $\mathrm{Y}_{500}$ were in a very good agreement. Thus, they used the predicted $\mathrm{Y}_{500}$ values for the non-halo clusters with no $\mathrm{Y}_{S Z}$ reported in the PSZ1 catalogue on the $\mathrm{P}_{1.4}-\mathrm{Y}_{500}$ plot. Cassano et al. (2013) supplemented their claim about the occurrence of the bi-modality by investigating the correlation between $\mathrm{M}_{500}$ and $\mathrm{P}_{1.4}$ and reported a similar bimodal behaviour for the halo and non-halo clusters in the $\mathrm{P}_{1.4}-\mathrm{M}_{500}$ plane. They used the $\mathrm{Y}_{500}-\mathrm{M}_{500}$ correlation (see Arnaud et al., 2010) to estimate the $\mathrm{M}_{500}$ values for the non-halo clusters undetected with Planck to create the $\mathrm{P}_{1.4}-\mathrm{M}_{500}$ plot.

Cassano et al. (2013) found a clear bi-modality in the $\mathrm{P}_{1.4}-Y_{S Z}$ plane and pointed out that the power of the non-halo clusters were 5-7 times less than the predicted value from the derived correlation in their work. They also mentioned that, according to the fit, their GMRT observations for some of the clusters with lower $Y_{S Z}$ were not sensitive enough to detect radio halos with the predicted power from their previous correlation. Although Basu (2012a) reported that they have not observed a strong bi-modality in the $\mathrm{P}_{1.4}-\mathrm{Y}_{S Z}$ plane, Cassano et al. (2013) argued that the sample of non-detections that had been investigated by Basu (2012a) was statistically incomparable. They stressed this was because only four out of 20 non-halo clusters in the GRHS had available SZ measurements in the Planck ESZ catalogue.

To investigate the bi-modality, we have cross-correlated our observed sample with the PSZ2 catalogue but only three out of our 15 observed clusters had a measured integrated Compton parameter ${ }^{5}$. This can be easily understood since the majority of our observed clusters are low-mass systems and hence remained undetected in the Planck observations. We employed the same approach as Cassano et al. (2013) and used the estimated $\mathrm{Y}_{500}$ and $\mathrm{M}_{500}$ values presented by Arnaud et al. (2010) for the remaining 12 non-halo clusters in our sample. We show new plots of the $\mathrm{P}_{1.4}-\mathrm{Y}_{S Z}$ and $\mathrm{P}_{1.4}-\mathrm{M}_{500}$ correlations including all detected halos and our derived upper limits in Figure 7.3. Ac-

\footnotetext{
${ }^{5}$ We also cross matched the observed clusters by Farnsworth et al. (2013) with the PSZ2 catalogue but none of them had SZ measurements.
} 

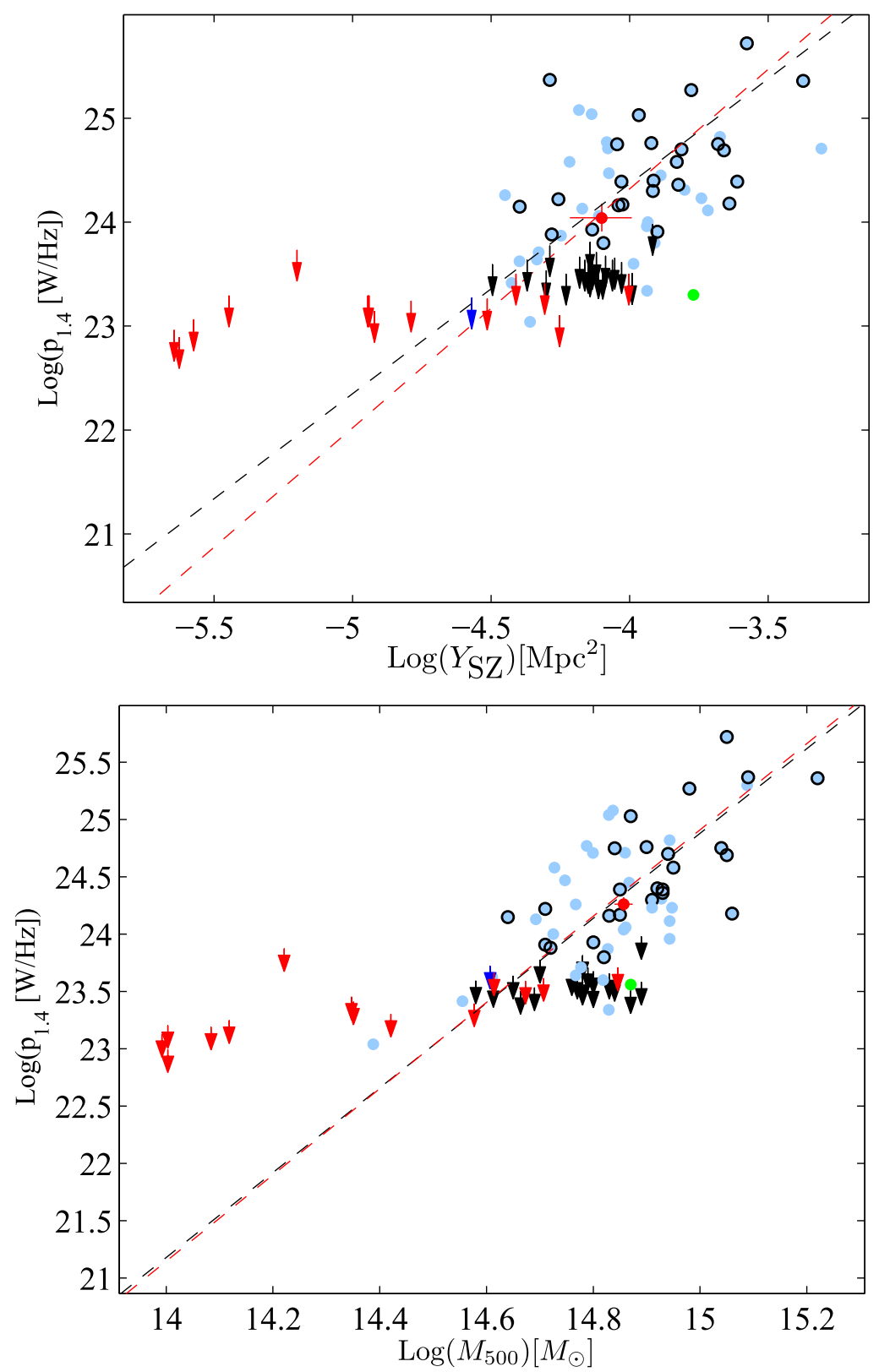

Figure 7.3: The upper limits are overlaid on the $\mathrm{P}_{1.4}-\mathrm{Y}_{S Z}$ and $\mathrm{P}_{1.4}-\mathrm{M}_{500}$ planes from top to bottom, respectively. The upper limit of non-halo clusters in this work are shown with red arrows. Black arrows indicate the upper limit values in the GRHS + EGRHS and blue arrow represents the upper limit derived by Russell et al. (2011). The black dashed line is the fit determined by Cassano et al. (2013) and red dashed line is the fit derived in this work. The blue dots are all the halos in the literature and blue dots enclosed with black circles are the halos used to derive black dashed line fit. The green dot shows the first direct halo detection in the "off-state" region (Bonafede et al., 2015). For the sake of clarity we only have shown the error bars of the halo in A3888, however, the errors of the power, $\mathrm{Y}_{S Z}$ and $\mathrm{M}_{500}$ of all the radio halos are reported in Appendix B.1.

cording to this plot, there were merely three clusters (RXCJ0645.4-5413, RXCJ2014.82430 and RXCJ2218.6-3853) with upper limits less than the predicted power and the remaining upper limits reside well above the predicted power values in the $\mathrm{P}_{1.4}-Y_{S Z}$ plane. This trend was equally valid considering each of the fits (derived from this work 

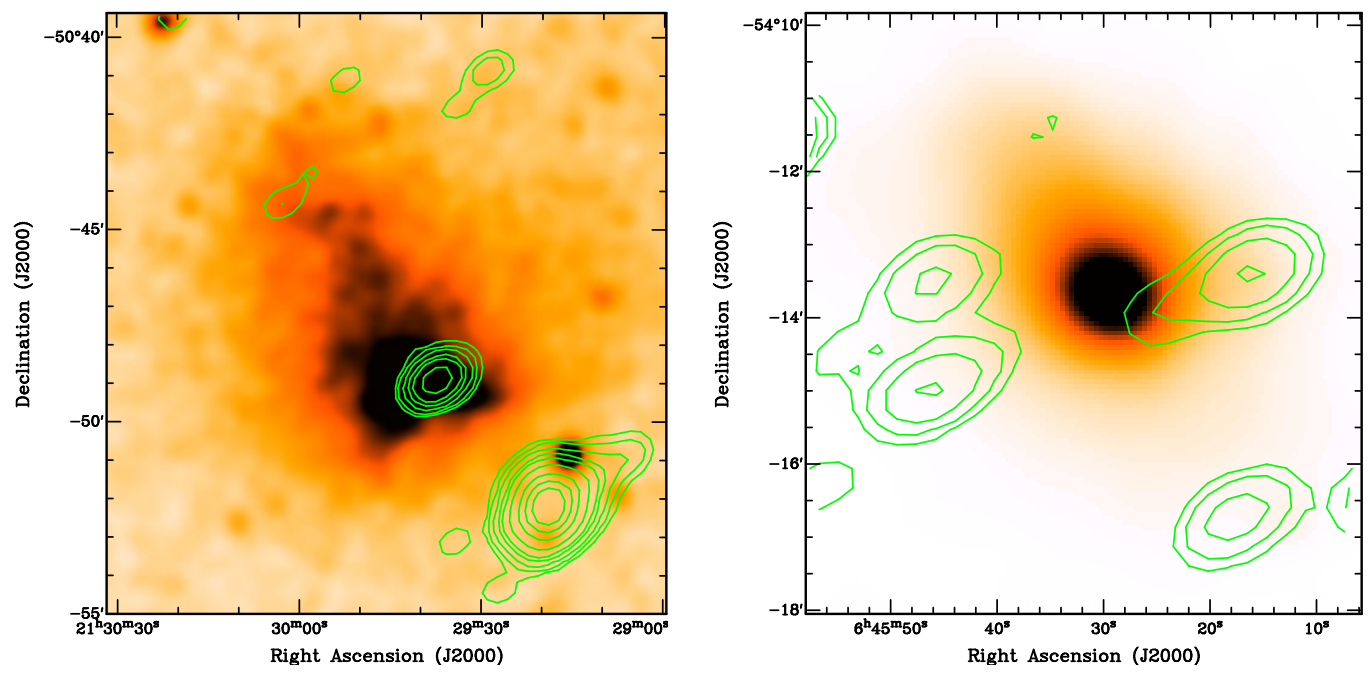

Figure 7.4: Radio emission observed in the low resolution images at $1.4 \mathrm{GHz}$ are superimposed on the X-ray observations of clusters RXCJ2129.8-5048 (A3771) and RXCJ0645.4-5413 (A3404), respectively, from left to right. Contours are shown at levels of $[1,2,4,8, \ldots] \times 4 \sigma_{r m s}$.

or the fit presented by Cassano et al., 2013) and this discrepancy between the upper limits and predicted power values was more pronounced for our X-ray under-luminous clusters. This finding might signify that our ATCA observations have not provided sufficient sensitivity to be able to detect radio halos with the predicted power less that $10^{23}$ W Hz${ }^{-1}$ at $1.4 \mathrm{GHz}$.

At odds with Cassano et al. (2013), we cannot claim that there is a visible segregation in the $\mathrm{P}_{1.4}-\mathrm{Y}_{S Z}$ plane. On the other side, due to the lack of measured SZ signal for low mass halo clusters, both fits in the $\mathrm{P}_{1.4}-\mathrm{Y}_{S Z}$ plane solely represent massive clusters and therefore the observed correlation is subject to a significant change once the SZ measurements of the low mass clusters become available. Figure 7.3 bottom plot shows the $\mathrm{P}_{1.4}-\mathrm{M}_{500}$ plane. According to this plot the majority of our upper limits reside above or close to both of the fits, again suggesting that our radio observations were insensitive to radio halos with such low powers. However, as mentioned earlier regarding the radio/SZ bi-modality, the $\mathrm{P}_{1.4}-\mathrm{M}_{500}$ plot is highly representative of massive clusters. Thus, our bi-modality investigation has remained inconclusive.

\section{§7.6. Lack of a Halo in A3771 and A3404}

Clusters RXCJ2129.8-5048 (A3771) and RXCJ0645.4-5413 (A3404) were the most disturbed clusters in our REXCESS sub-sample. As mentioned earlier, our radio observations with the ATCA have not revealed any radio halos in these merging clusters. In Figure 7.4, low-resolution radio contours are overlaid on the X-ray images of clusters RXCJ2129.8-5048 and RXCJ0645.4-5413, respectively from left to right. The lack of GRHs in disturbed clusters have been previously reported in the literature; for instance, 
Farnsworth et al. (2013) found no halo emission in the merging cluster Abell 2056 and Russell et al. (2011) also reported that the absence of a radio halo in the merging cluster A2146. Russell et al. (2011) claimed that A2146 was an ideal candidate to host a GRH due to its high X-ray luminosity and disturbed morphology, and suggested that the lack of radio halo is probably caused by the lower mass of this cluster.

We note that A3771 has an extremely low X-ray luminosity of only $0.76 \times 10^{44} \mathrm{erg}$ $\mathrm{s}^{-1}$. On account of the lack of radio halo statistics with minimal selection effects, it is not clear to what power levels, the halo detection in such low X-ray luminosity clusters is anticipated.

According to our work and the work presented by Farnsworth et al. (2013) in which all their observed clusters were merging and the majority of them had low X-ray luminosities, the lack of a radio halo at $1.4 \mathrm{GHz}$ might be suggestive that the generation of the radio halos is more affected by the mass of the clusters than previously thought.

As opposed to A3771, Abell 3404 is one of the most plausible candidates to harbour a radio halo in our observed sample. This cluster has high $\mathrm{X}$-ray luminosity of $7 \times 10^{44}$ $\mathrm{erg} \mathrm{s}^{-1}$ and is disturbed. This cluster is even more massive than A3888 which hosts a GRH and its tapered low-resolution image has a very close rms noise compared to the radio map of A3888. For these reasons, the absence of radio halo in A3404 is quite intriguing.

The presence of USS halos (Dallacasa et al., 2009) and the re-acceleration model of halo generation suggest that there are some non-detected radio halos in $\mathrm{GHz}$ observations at the sensitivity level of our current telescopes and follow-up low frequency observations with the MWA-II might be helpful to detect a possibly very faint or USS halo in A3771 and A3404.

\section{$\S$ 7.7. Summary and Discussion}

In this chapter, we followed the procedure employed by Brunetti et al. (2007) and Pratley (2012) to inject $1 \mathrm{Mpc}$ diameter elliptical fake halos into the original uv-data to derive the upper limits in our non-halo clusters. We then gathered the information of 62 halos (61 published halos to date and this work) to update the radio/X-ray correlation presented by Cassano et al. (2013). Our fit to all data is significantly different from the fit derived by Cassano et al. (2013). However, this was not surprising since the sample used by Cassano et al. (2013) only contained clusters with high X-ray luminosity. We also derived new fits for the radio/SZ and radio/mass correlations but our fit was consistent with the fits presented by Cassano et al. (2013). This was mainly because of the lack of SZ and mass measurements for lower mass clusters in the Planck cluster catalogue (PSZ2). 


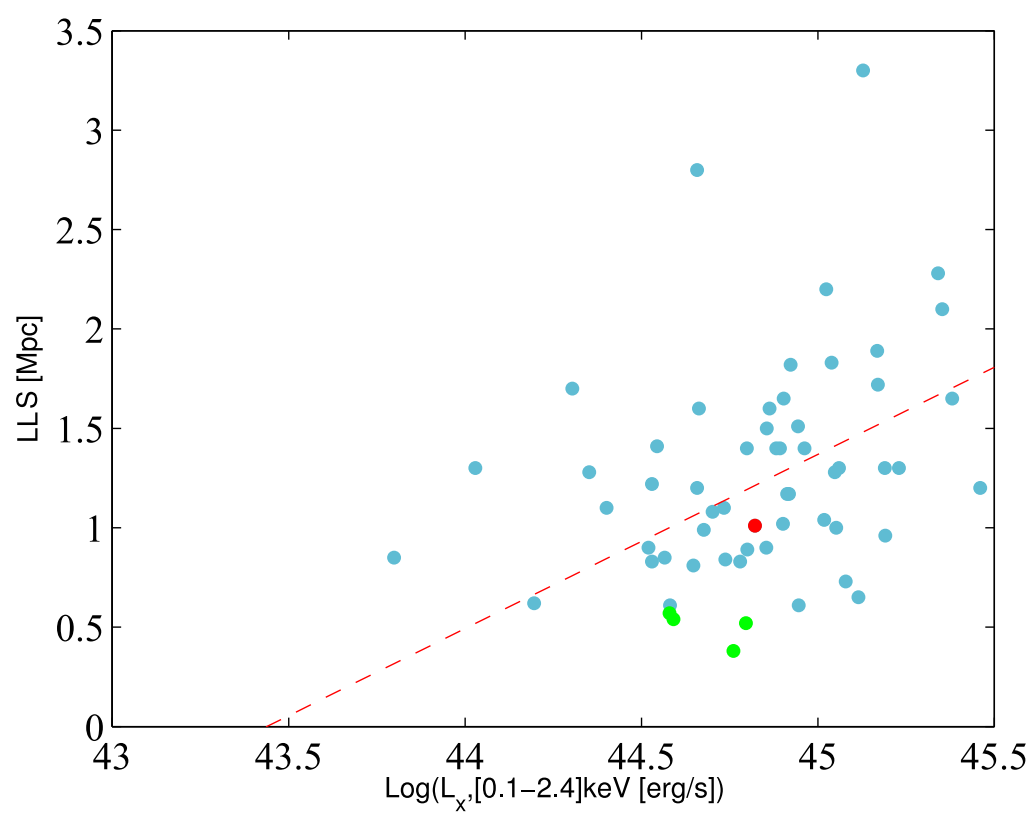

Figure 7.5: All dots represent the radio halos from the literature. The red dot is the halo in A3888. The green dots represent the outliers which reside in the "radio-quiet" region of the $\mathrm{P}_{1.4}-\mathrm{L}_{X}$ plane. The red dashed line indicates our fit to the data with $\mathrm{L}_{X}>10^{44.5} \mathrm{erg} \mathrm{s}^{-1}$.

We also have indicated our upper limits alongside the upper limits derived in the EGRHS+ GRHS and the GBT project on the $\mathrm{P}_{1.4}-\mathrm{L}_{X}, \mathrm{P}_{1.4}-\mathrm{M}_{500}$ and $\mathrm{P}_{1.4}-\mathrm{Y}_{500}$ planes. This allowed us to investigate the correlations between the thermal and non-thermal components of the clusters with or without halos and to investigate the matter of bimodality in the distribution of the clusters. The location of our halo in A3888 is in agreement with the fit derived in this work and the correlation presented by Cassano et al. (2013) in all the scaling relations plots.

One of implications of the re-acceleration halo generation scenario, which is currently the most preferable model of GRH generation, is the bimodal behaviour of clusters in terms of their radio luminosity. According to this model, galaxy clusters with no radio halos populate the "radio quite" region of the $\mathrm{P}_{1.4}-\mathrm{L}_{X}, \mathrm{P}_{1.4}-\mathrm{M}_{500}$ and $\mathrm{P}_{1.4}-\mathrm{Y}_{500}$ planes. The occurrence of merging can trigger halo generation and as a result, clusters move towards the predicted locations ("radio loud" region) on the correlation and then gradually move downwards when the parent cluster becomes more relaxed. Accordingly, the size of the halo increases whilst it is moving upwards and then halo gradually dissipates whilst the location moves downwards (Brunetti et al., 2009).

The nature of the lack or presence of bimodal behaviour of the clusters with $\mathrm{L}_{X}<$ $10^{44.5} \mathrm{erg} \mathrm{s}^{-1}$ in the aforementioned planes has not very well investigated. The majority of clusters in our sample have $\mathrm{L}_{X}<10^{44.5} \mathrm{erg} \mathrm{s}^{-1}$ but due to lack of sensitivity in our radio maps, we cannot determine whether the bi-modality exists or not.

In all three correlations presented in the $\mathrm{P}_{1.4}-\mathrm{L}_{X}, \mathrm{P}_{1.4}-\mathrm{Y}_{500}$ and $\mathrm{P}_{1.4}-\mathrm{M}_{500}$ planes, 
there are a few outliers which settle in the radio quite region. We found that the majority of outliers in the 62 halos discovered to date reside in the X-ray luminosity range $10^{44.5}<\mathrm{L}_{X}<10^{50} \mathrm{erg} \mathrm{s}^{-1}$. The outliers are shown with green dots in Figure 7.5. The LLS size of all the outliers is less than $570 \mathrm{kpc}$ whilst the remaining radio halos in this X-ray luminosity bin have the measured LLS greater than $750 \mathrm{kpc}$. According to the scaling relation between the LLS and $\mathrm{L}_{X}$ of halo clusters (see Figure 7.5), these outliers are expected to have a much greater linear size. This might suggest that the outlier halo clusters are at the early/later stage of their life-time. However, there are other radio halos with a measured LLS much greater than the predicted value by the Cassano et al. (2013) correlation and this scatter is more pronounced in the region in which the halo clusters are X-ray under-luminous. Thus, investigation of this transition in the life-time of the clusters requires a greater sample of halos in different X-ray luminosity bins.

Lack of a radio halo in our most disturbed clusters Abell 3771 And Abell 3404 was particularly intriguing. A3771 is disturbed and X-ray under-luminous and the anticipated halo power for this cluster is much less than its derived upper limit. As a result it is not clear whether the radio halo does not exist or it is buried under the noise level of our image. However, in A3404, the derived upper limit is noticeably less than the predicted halo power value. Thus, we can infer that if the current correlations are representative, no radio halo exists at $1.4 \mathrm{GHz}$. We suggest that low frequency radio observations with MWA-II might detect possible USS halos in these clusters.

Upcoming radio telescopes with unprecedented sensitivity such as SKA are expected to dramatically increase our ability to detect low surface brightness halos in clusters with low X-ray luminosity. We find that although merging is essential to trigger halo generation, it is not the only needed parameter to generate a radio halo in all merging clusters. Cluster mass appears to be the other key component in the radio halo generation. However, due to the limits of our current radio telescopes and extreme selection effects in current halo surveys, neither of the arguments above can be definitely made. 


\section{§. Examining the Magnetic Field in the Jets of the Head-tail Galaxy SJ212919-505226}

\section{$\S 8.1$. Introduction}

The generation of the observed well-collimated jets of plasma in AGNs can only be explained by the magnetic field surrounding the accretion disc and the central black hole. Thus, the presence of magnetic fields on kilo-parsec scales is vital to explain the jet formation and evolution. In addition, the magnetic field is believed to play a crucial role in maintaining the collimation and direction of the jets. Radio observations of the jets have revealed that there are two types of magnetic fields in the AGN jets; 1) regular magnetic fields; and 2) helical magnetic fields. The regular magnetic fields have longitudinal and toroidal (perpendicular) components which drop proportionally to $\mathrm{r}^{-2}$ and $\mathrm{r}^{-1}$, respectively, thus, the toroidal component becomes more effective at larger distances from the core of AGN. However, the toroidal component can be amplified and ordered at smaller distances from the core in the case of pre-existing seed fields. In contrast to longitudinal magnetic fields, which are commonly seen in AGN jets, toroidal magnetic fields have been rarely observed in the AGN jets. Based on simulations (Pudritz et al., 2012; McKinney et al., 2014) it is believed that the rotation of the central black hole and the accretion disc result in more complex magnetic field structures known as "helical magnetic field" (see Hovatta et al., 2012a and references therein), however, due to a small number of helical field detections our knowledge about these helical fields and their profile along the AGN jets is quite limited.

The magnetic field direction in the radio jets are inferred by rotating the observed projected electric vector position angle (EVPA) by $90^{\circ}$. In interferometry, we observe the vector sum of the two E-fields projections (associated with the LOS and projected components of the B-field) on to the plane of sky. Rotation Measure (RM) analysis is the only way to distinguish between these projected components. RM-analysis of the polarised radio sources can be done by observing the source at multiple frequencies which are close in frequency space. Unfortunately, the $n \pi$ ambiguity and depolarisation can heavily limit the RM investigations.

In recent years, broadband polarimetry and particularly RM studies of AGNs have received more attention in the community (O'Sullivan et al., 2012; Gaensler et al., 2015). On account of wide-band frequency observations, more reliable RM maps have become available and as a result, investigating the magnetic field of AGNs has become 
more promising and the number of toroidal and helical magnetic fields has increased (O'Sullivan et al., 2012; Hovatta et al., 2012b; Gabuzda et al., 2015) with around 25 possible detections found in pc-scale jets.

The recent upgrade of the ATCA to CABB system has provided an opportunity to explore the polarisation properties of AGN over a wide-band of frequencies. However, to date only a small number of projects (e.g. O'Sullivan et al., 2012; Shimwell et al., 2014) have exploited the virtue of the broadband polarimetry offered by the ATCA. Here, we aim to use this superb capability of the ATCA to investigate the broadband polarimetic data of the HT galaxy SJ212919-505226 in Abell 3771 (A3771).

Detection of an HT galaxy with well-resolved jets in high-resolution data with a $2 \mathrm{GHz}$ bandwidth make this HT galaxy as an ideal candidate to carry out a detailed analysis of the magnetic field of the source by means of broadband polarimetry, RM synthesis and multi-frequency spectral index analysis.

In this chapter, we discuss the radio observations and imaging of the detected HT galaxy. The spectral index and spectral index error maps are presented. The Faraday RM of the HT galaxy is derived Faraday rotation measure synthesis. The magnetic field direction along the jets of the HT galaxy are also investigated. Possible explanations regarding the observed peculiar magnetic field structure in the HT galaxy are then discussed.

Throughout this chapter we use a $\Lambda$ CDM cosmology with $\mathrm{H}_{0}=67 \mathrm{kms}^{-1} \mathrm{Mpc}^{-1}$, $\Omega_{m}=0.32$ and $\Omega_{\Lambda}=0.68$ (Planck Collaboration et al., 2013). According to above assumptions, the angular scale is $10.63^{\prime} / \mathrm{Mpc}$ at the redshift of this cluster $(\mathrm{z}=0.0796)$.

\subsection{Radio Observations and Stokes I Imaging}

As mentioned earlier in Chapter 4, low resolution imaging of the $1.4 \mathrm{GHz}$ pre-CABB data for A3771 alongside the SUMSS archival image at $843 \mathrm{MHz}$ revealed an extended radio source at the edge of the cluster's X-ray emission (see $\S 4.3$ ). The combination of pieces of evidence such as the location of the detected extended source and the lack of the optical counterpart at the location of peak brightness of the source have suggested that the radio object could be a radio relic candidate (see Figure 4.2).

Follow-up radio observations were carried out in the 6A, H214 and H168 array configurations using CABB mode at a central frequency of $2.1 \mathrm{GHz}$ with a $2 \mathrm{GHz}$ bandwidth. Details of the observations of A3771 are given in Table 4.3. The high resolution image $\left(\sim 6^{\prime \prime}\right)$ revealed that this radio relic candidate is, in fact, a head tail galaxy with two well-separated jets. Figure 8.1 shows the high resolution mosaic image using all available $\mathrm{CABB}$ data at $2.1 \mathrm{GHz}$. Using a more compact array configuration allowed us to detect excess diffuse emission of the tailed radio galaxy across the lobes. 

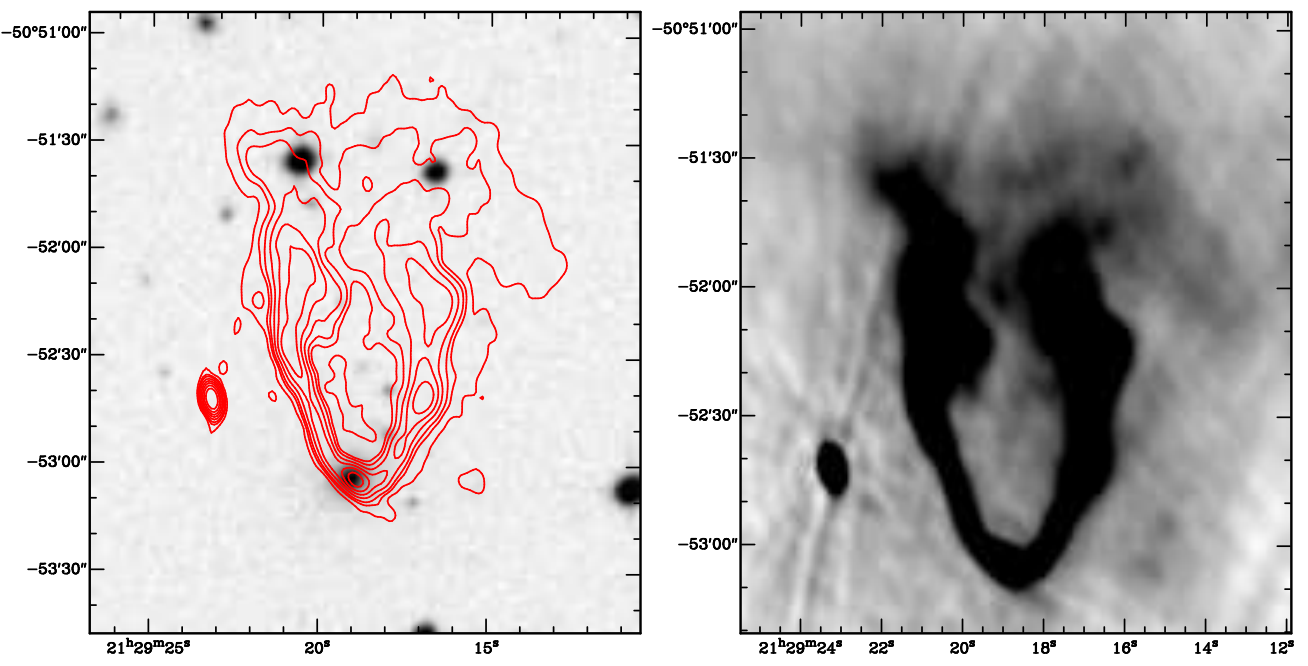

Figure 8.1: Right panel: high resolution Stokes I image $\left(6^{\prime \prime} \times 4^{\prime \prime}\right)$ of the HT galaxy in A3771. Red contours represent the mosaiced CABB data at $2.1 \mathrm{GHz}$. All the contours are superimposed on the DSS blue image. The contours levels are at $1,2,4, \ldots \times 4 \times 45 \mu \mathrm{Jy}_{\text {beam }}{ }^{-1}$. The optical counterpart is clearly visible. Left panel: radio emission of the HT galaxy. The mosaic CABB radio image is on the bachground.

Details of the Stokes I images are given in Table 4.2 and Table 4.5. For the purpose of the data reduction and imaging, we have used the procedures explained in Chapter 3. The minimum and maximum baseline lengths were $31 \mathrm{~m}$ (pre-CABB data) and $6 \mathrm{~km}$ (CABB data), respectively, which corresponds to angular scales between $\sim 30^{\prime}$ at 1.1 $\mathrm{GHz}$ and $\sim 4^{\prime \prime}$ at $3.1 \mathrm{GHz}$. The combined array configuration offered a wide-range of spatial frequencies and allowed us to probe different spatial scales of this tailed radio galaxy.

The total observing time was 24.8 hours including both the $\mathrm{CABB}$ and pre-CABB observations. The largest angular size of the detected HT galaxy was $85^{\prime \prime}$ measured at $3 \sigma_{r m s}$ at $1.4 \mathrm{GHz}$ in the coadded Stokes I image. This angular size corresponds to the largest linear size of $134 \mathrm{kpc}$. The total flux and total polarised flux were measured as $304.75 \mathrm{mJy}$ and $23.58 \mathrm{mJy}$, respectively at $1.4 \mathrm{GHz}$ with a $300 \mathrm{MHz}$ bandwidth in the region enclosed by the $3 \sigma_{\text {rms }}$ contour.

The optical counterpart associated with this tailed radio galaxy is an elliptical galaxy with redshift 0.0799 (Corwin and Emerson, 1982). The detected HT galaxy is a member of A3771 ( $\mathrm{z}=0.0796)$ and is located $7^{\prime}(658 \mathrm{kpc})$ away from the centre of the cluster. We searched the NED for available redshifts in two degrees radius around A3771 and found only 103 measured redshifts available from the literature, from which, merely nine galaxies had redshifts in the range of the cluster (0.07-0.09). On account of a limited number of measured redshifts, the optical analysis and substructure test of the parent cluster were not possible. 
Table 8.1: Top: details of Stokes I images made from pre-CABB and CABB data at 1.4 and 2.1 $\mathrm{GHz}$, respectively. We made two sets of Stokes I images from CABB data since the pointing of one the CABB observations was different (see Table 4.3). The CABB data with identical pointings were used in the spectral index analysis. Bottom: details of the polarisation $300 \mathrm{MHz}$ sub-band images using only CABB data created at 1.4 and $2.4 \mathrm{GHz}$.

\begin{tabular}{ccccl}
\hline $\begin{array}{c}\text { Frequency } \\
\text { MHz }\end{array}$ & $\begin{array}{c}\text { Beam FWHM, PA } \\
" \times{ }^{\prime \prime},{ }^{\circ}\end{array}$ & $\begin{array}{c}\sigma_{I} \\
\mu \mathrm{Jy} \mathrm{beam}^{-1}\end{array}$ & $\begin{array}{c}\text { Band-width } \\
\mathrm{MHz}\end{array}$ & Notes \\
\hline 2100 & $7 \times 4,8$ & 56 & 2000 & Dec, Mar CABB, Figure 8.3 \\
2100 & $6 \times 4,11$ & 45 & 2000 & Jul, Dec, Mar mosiac-CABB, Figure 8.1 \\
\hline $\begin{array}{c}\text { Frequency } \\
\text { MHz }\end{array}$ & $\begin{array}{c}\text { Beam FWHM, PA } \\
" \times{ }^{\prime \prime},{ }^{\circ}\end{array}$ & $\begin{array}{c}\sigma_{Q, U} \\
\mu \mathrm{Jy}^{-1}\end{array}$ & $\begin{array}{c}\text { Band-width } \\
\mathrm{MHz}\end{array}$ & Notes \\
\hline 1400 & $9 \times 5,7$ & 50 & 300 & Dec, Mar CABB, Figure 8.2 and 8.8 \\
2400 & $5 \times 3,10$ & 34 & 300 & Dec, Mar CABB, Figures 8.2 and 8.8 \\
\hline
\end{tabular}

\section{$\S$ 8.3. Polarimetry}

In the course of observations with the ATCA, all the linear feed measurements (XX, YY, $\mathrm{XY}, \mathrm{YX}$ ) were correlated to achieve full polarimetric sensitivity. The off-axis polarisation leakages of the ATCA observations at $16 \mathrm{~cm}(2.1 \mathrm{GHz})$ at the full width half-power (FWHP) point are reported to vary from $\sim 1 \%$ at $1.1 \mathrm{GHz}$ to $6 \%$ at $3.1 \mathrm{GHz}$. Given that the primary beam FWHP at $2.1 \mathrm{GHz}$ is $22.3^{\prime}$ and our HT galaxy is located $7^{\prime}$ away from the pointing centre, we do not expect significant instrumental off-axis leakages. Thus, we have not corrected for the off-axis leakages in our polarisation analysis.

In the polarimetry, we used the combined CABB data observed in December and March which was temporally close and had the same pointing centres. The linear Stokes parameters $\mathrm{Q}$ and $\mathrm{U}$ were used to derive two $300 \mathrm{MHz}$ sub-band polarisation, polarisation angle (PA) and the polarisation angle error images at 1.4 and $2.4 \mathrm{GHz}$. The details of the polarisation images of the HT galaxy at 1.4 and $2.4 \mathrm{GHz}$ are given in Table 8.1. The PA images were masked where the PA error was greater than $10^{\circ}$ or the polarisation and Stokes I images had brightness values below the $3 \times \sigma_{r m s}$. We made use of the PA images to infer the direction of the magnetic field of the HT galaxy. In addition to PA images, the fractional polarisation images of the HT galaxy at $1.4 \mathrm{GHz}$ and 2.4 $\mathrm{GHz}$ were also created. Figure 8.2 shows the fractional polarisation of the HT galaxy at $1.4 \mathrm{GHz}$ (top plot) and $2.4 \mathrm{GHz}$ (bottom plot). The masked pixels are identical to the masked pixels in the PA images. Interestingly, an increase of the fractional polarisation towards the outer edge is evident at both frequencies. 

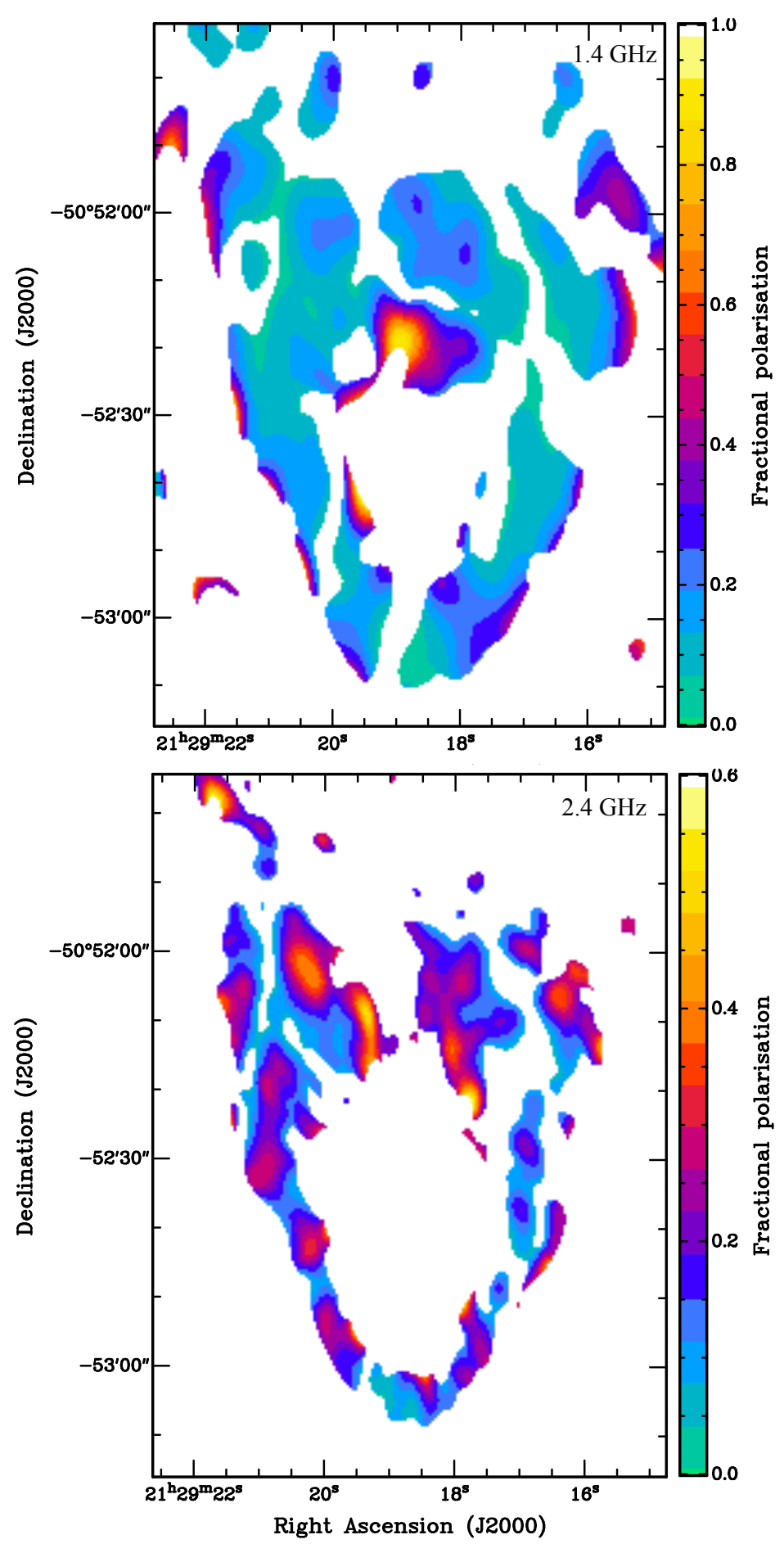

Figure 8.2: Fractional polarisation image of the HT galaxy at $1.4 \mathrm{GHz}$ and $2.4 \mathrm{GHz}$ are shown in top and bottom plots, respectively. The masked pixels are identical to the masked pixels in the PA image. 


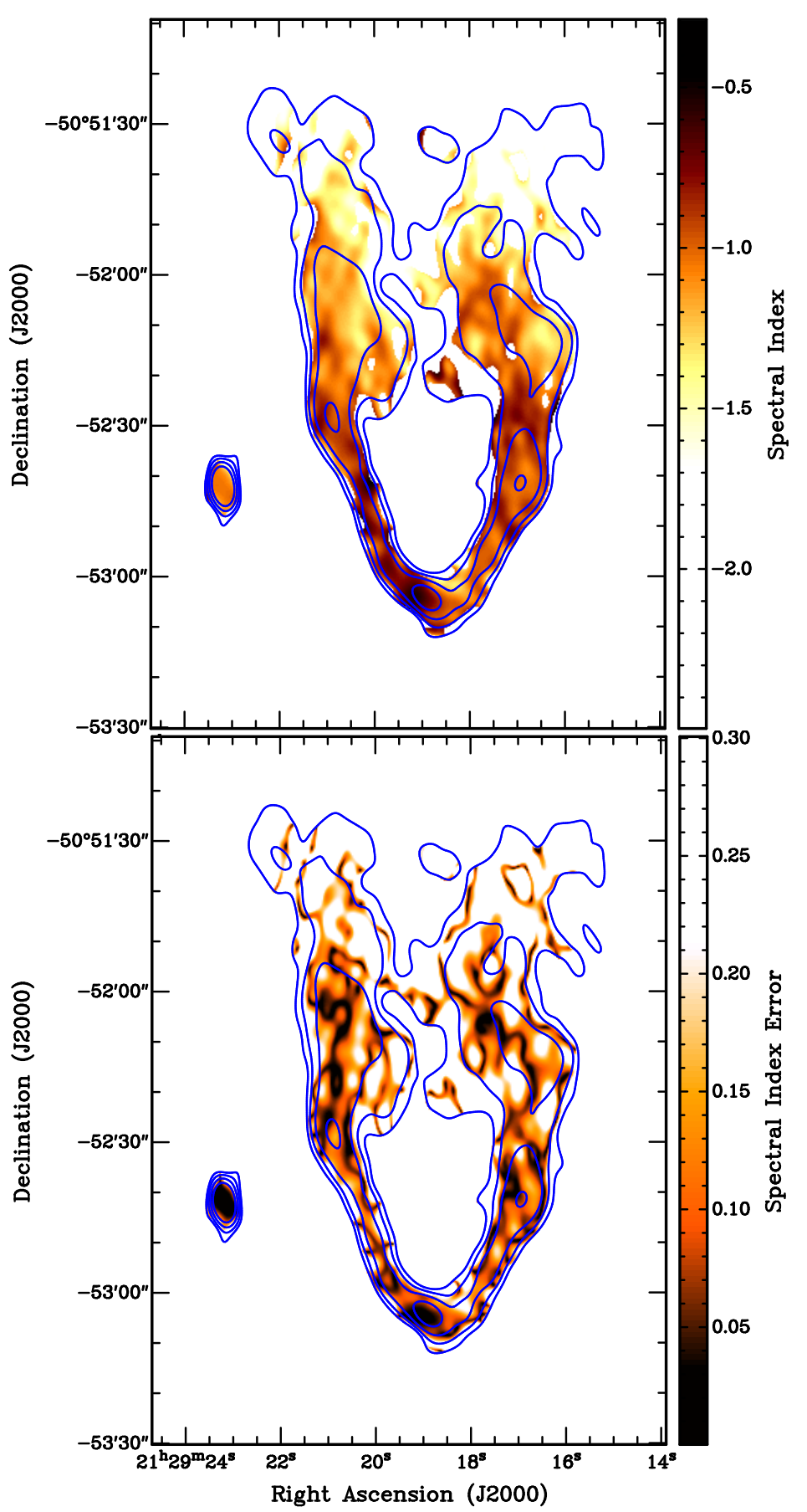

Figure 8.3: Top Panel: The spectral index map of HT galaxy. Bottom panel: The spectral index error map of the HT galaxy. The maps are created using the wide-band multi-scaling CLEANing method in CASA. The pixels of the both maps are blanked where the error value was greater than 0.3 . The blue contours represent the total brightness image and the contour levels are at $1,2,4, \ldots \times 4 \times 45 \mu \mathrm{Jy}_{\text {beam }}{ }^{-1}$. 


\section{§.4. Spectral Index Map of SJ212919-505226}

Radio galaxy spectra are mostly described with a simple power-law form given as $\mathrm{s}$ $\propto v^{-\alpha}$ where $\mathrm{s}$ is the total flux at frequency $v$ and spectral index $\alpha$.

A flatter spectral index is commonly observed in hot spots, knots and the cores of AGNs while steeper spectral indices are expected in the extended diffuse components such as in the lobes and along the jets where the electron population age in the plasma. The synchrotron emitting plasma has lower synchrotron and IC losses, particularly at lower redshifts and lower frequencies, thus, it is more likely that the spectral indices are representative of the intrinsic energy distribution of the highly energetic and relativistic electrons in the plasma.

We used the wide-band multi-frequency and multi-scaling imaging method in the data reduction software, CASA, to derive the spectral index map of the HT galaxy. We preferred to use two Taylor polynomial terms (nterms=2) to describe the sky brightness as a function of frequency. Using two Taylor terms, the spectral brightness can be expressed as follows:

$$
I_{v}=I_{0}+I_{1}\left(\frac{v-v_{0}}{v_{0}}\right) .
$$

where $I_{0}$ and $I_{1}$ are the images which represent the first two coefficients in the Taylor expansion and $v_{0}$ is the reference frequency in the polynomial fit. If we take spectral index into account, the sky brightness is described as:

$$
I_{v}=I_{v_{0}}\left(\frac{v}{v_{0}}\right)^{\alpha}
$$

then by comparing the two equations above, we obtain $I_{v_{0}}=I_{0}$ and $\alpha=\frac{I_{1}}{I_{0}}$. The spectral index map, spectral index error and $\mathrm{I}_{0}$ are the resultant images in the multi-frequency imaging process. The wide-band multi-frequency imaging has some advantages over the traditional method in which several images with smaller bandwidth are used to create the spectral index map. This method can offer more accurate and reliable spectral map as data with larger frequency-coverage is used. A detailed description of the multifrequency multi-scale CLEANing method can be found in (Rau and Cornwell, 2011).

The measured errors of the spectral index are proportional to the bandwidth, $\mathrm{u}-\mathrm{v}$ coverage and the signal-to-noise ratio. We have avoided using higher Taylor terms since it requires a higher level of signal-to-noise ratio in the total brightness map. Shimwell et al. (2014) adopted the same approach to determine the spectral index map of the radio halo detected in Bullet cluster. They confirmed that this method was in line with the traditional narrow band method which was used in previous publications.

It is essential to investigate whether the largest detected object is smaller than the largest detectable structure by the telescope over the full band. The spatial frequencies 
corresponding to the scale of our HT at $1.1 \mathrm{GHz}$ should be larger than the minimum measured spatial frequency at the higher-end of the band $(3.1 \mathrm{GHz})$, otherwise, the higher frequency part of the bandwidth should be excluded from the spectral index analysis. This treatment is crucial because the measured spectral indices suffer from incomplete total flux measurement at the higher frequency end of the band and thus the $\alpha$ values are not reliable.

We used the December 2011 and March 2012 CABB datasets to create the spectral index map. The dataset observed in July 2011 was excluded from our spectral index analysis since the data had a different pointing and currently CASA is unable to perform the multi-frequency multi-scale CLEANing in mosaic mode. We verified whether the total flux measurement of the HT galaxy was possible at the higher-frequency end of the $\mathrm{CABB}$ band $(3.1 \mathrm{GHz})$ and fortunately, there was no issue with using the full band $(1.1-3.1 \mathrm{GHz})$ in our spectral index analysis.

We made use of another superior capability of CASA in the CLEANing procedure. The multi-scale CLEANing was also applied to avoid the errors which are induced by the traditional point source CLEANing during the de-convolution process for extended radio sources. The image was CLEANed at the scales of zero (point source), three and six times the beam FWHM to cover different observed structures in the HT galaxy. We then used task "WIDEBANDPBCOR" in CASA to preform the primary beam correction on the resultant maps. In this task, the spectral variation of the primary beam is determined by fitting a polynomial (nterms=2) to correct the flux measurements and then the spectral index and spectral index error maps are re-created. In Figure 8.3 top plot, the spectral index map of the HT galaxy is shown. The spectral index values are more flat around the galaxy core but become steeper along the jets, lobes and in the diffuse region between the jets. The spectral indices have higher corresponding errors due to the lower $\mathrm{S} / \mathrm{N}$ ratio at the edges. The spectral index error map of the HT galaxy is also shown in Figure 8.3, bottom plot. The spectral index and spectral index error maps are masked if the error was greater than 0.3.

\section{§8.5. Rotation Measure of SJ212919-505226}

For the purpose of RM synthesis, we have used the software developed by Brentjens and de Bruyn, 2005 and which is publicly available ${ }^{1}$.

We first created the $\mathrm{Q}$ and $\mathrm{U}$ polarisation cubes where each had a $10 \mathrm{MHz}$ bandwidth (10 channels with channel-width of $1 \mathrm{MHz}$ ) spread over the full ATCA band with a robust weighting of zero. In the next step, the standard imaging procedure and primary beam correction were applied to each cube. Given that individual cubes had different

\footnotetext{
${ }^{1}$ website: http://www.astron.nl/ $\sim$ brentjens/index.html
} 


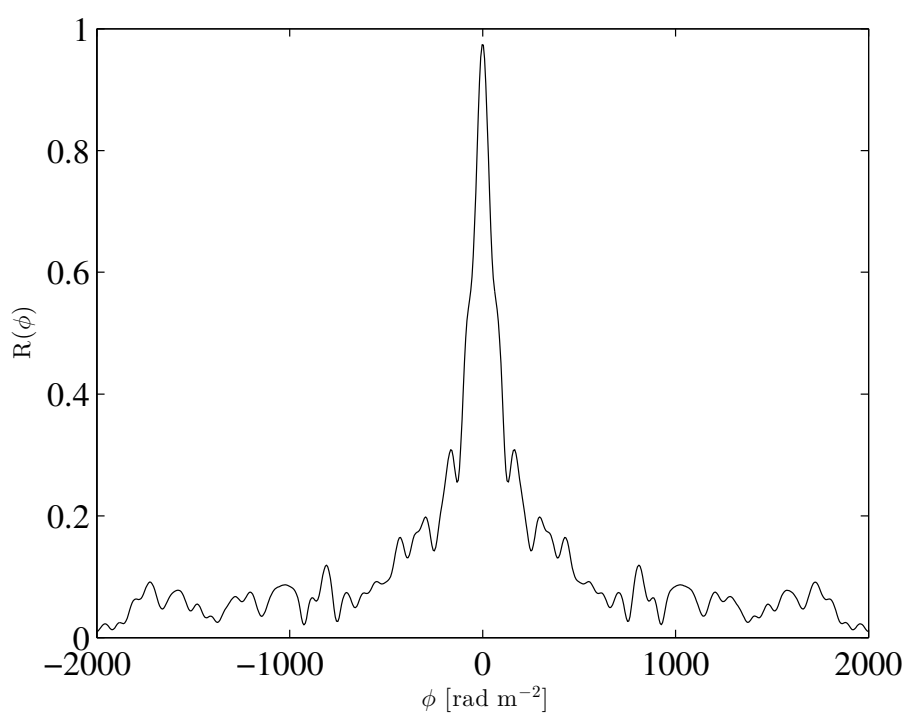

Figure 8.4: Rotation measure transfer function in our RM synthesis. The FWHM of the RMTF is $65.7 \mathrm{rad} / \mathrm{m}^{2}$.

resolution due to beam size variation across the $2 \mathrm{GHz}$ band, all the cubes were convolved to the resolution at the lowest frequency to match their final resolution. We have used task "regrid" with the option "noscale" to assure that the cell size of each plane in each cube does not scale with frequency, otherwise the RA and Dec of an identical pixel would be different along the cube. Finally, all the individual maps were combined using the task "imcat".

Unfortunately, some of the channels amongst this frequency range were removed since $100 \%$ of the channel was flagged. After flagging, the final Q and U cubes had 1506 planes and the minimum and maximum frequency were 1256 and $3123 \mathrm{MHz}$, respectively.

We then have adopted the RM synthesis method that was initially suggested by Burn (1966) and then extended by Brentjens and de Bruyn (2005). The RM synthesis is a unique tool to exploit the benefits of wide-band polarimetry and derive the Faraday dispersion function $\mathrm{F}(\phi)$ from $\mathrm{P}\left(\lambda^{2}\right)$ by using Fourier transform. This technique has some advantages over the traditional RM analysis. Since the polarisation signal is extracted over a wide range of frequency, the effect of bandwidth depolarisation is minimised. The other significant advancement in this method is that the RM analysis is much less affected by "n $\pi$ ambiguity".

The complex polarisation intensity as a function of Faraday depth is given by:

$$
F(\phi)=\sum_{j=1}^{N} w_{j} P_{j} e^{-2 i \phi\left(\lambda_{j}^{2}-\lambda_{0}^{2}\right)} / \sum_{j=1}^{N} w_{j} .
$$

$N$ is total number of channels in the dataset, $P_{j}$ is the complex polarisation, $F(\phi)$ is 
Table 8.2: RM Synthesis properties and detection capability over the CABB band at central frequency of $2.1 \mathrm{GHz}$. The table is adapted from (O'Sullivan et al., 2012).

\begin{tabular}{ccccc}
\hline \hline $\begin{array}{c}(1) \\
\begin{array}{c}\text { Frequency } \\
{[\mathrm{MHz}]}\end{array}\end{array}$ & $\begin{array}{c}(2) \\
\text { Resolution } \\
{[\mathrm{MHz}]}\end{array}$ & $\begin{array}{c}(3) \\
\delta \phi \\
{\left[\mathrm{rad} \mathrm{m}^{-2}\right]}\end{array}$ & $\begin{array}{c}(4) \\
\mathrm{max}^{-s c a l e} \\
{\left[\mathrm{rad} \mathrm{m}^{-2}\right]}\end{array}$ & $\begin{array}{c}(5) \\
\left|\phi_{\max }\right| \\
{\left[\mathrm{rad} \mathrm{m}^{-2}\right]}\end{array}$ \\
\hline $1256-3123$ & 1.0 & 65.7 & 340 & 13000 \\
\hline
\end{tabular}

Columns: (1) The frequency range in the input Q and U cubes; (2) Spectral resolution; (3) Resolution in Faraday depth space; (4) Maximum detectable Faraday thickness; (5) Maximum detectable Faraday depth.

the Faraday dispersion function, $j$ and $w_{j}$ are the channel number and weights (inverse square of the rms noise), respectively. To account for the incomplete Fourier sampling at $\lambda^{2}>0$ and lack of observing at $\lambda^{2}<0$, the measured complex polarisations are modified with a sampling function also known as weight function. More coverage in $\lambda^{2}$ space leads to a more sensitive constructed polarisation. The re-constructed Faraday dispersion function is given by

$$
F(\phi)^{\sim}=F(\phi) * R(\phi) .
$$

where the $R(\phi)$ is the rotation measure transfer function (RMTF). The enhanced $\lambda^{2}$ coverage with smaller central hole, significantly reduces the side lobes of the RMTF. Figure 8.4 shows the rotation measure transfer function (RMTF) with FWHM of 65.7 $\mathrm{rad} / \mathrm{m}^{2}$ in our RM cube.

Our final polarised cube (RM cube) was created over a wide range of Faraday depths staring from -2000 to $+2000 \mathrm{rad} / \mathrm{m}^{2}$ with the Faraday depth interval of $3.5 \mathrm{rad} / \mathrm{m}^{2}$. The Full width at half maximum (FWHM) of the RMTF in our constructed polarisation cube is $65.7 \mathrm{rad} / \mathrm{m}^{2}$.

Brentjens and de Bruyn (2005) claimed that the Faraday thick sources can only be resolved if:

$$
\lambda_{\min }^{2}<\Delta \lambda^{2}
$$

Owing to the broad frequency band offered in the CABB mode, our $\lambda^{2}$ coverage satisfies the above equation. The largest detectable scale is $340 \mathrm{rad} / \mathrm{m}^{2}$ and the maximum Faraday depth is $13000 \mathrm{rad} / \mathrm{m}^{2}$. The capabilities of our RM synthesis analysis in detecting Faraday thick sources are given in Table 8.2. In the RM synthesis process, the spectral index of the source was not taken into account (see Section 3 in Brentjens and de Bruyn, 2005). This treatment is vital if the polarisation is damping at higher frequencies due to the steep spectral index. We examined the Stokes Q and U intensities across the band for various pixels and sine waves were observed. Hence, the treatment mentioned in above was not required. 

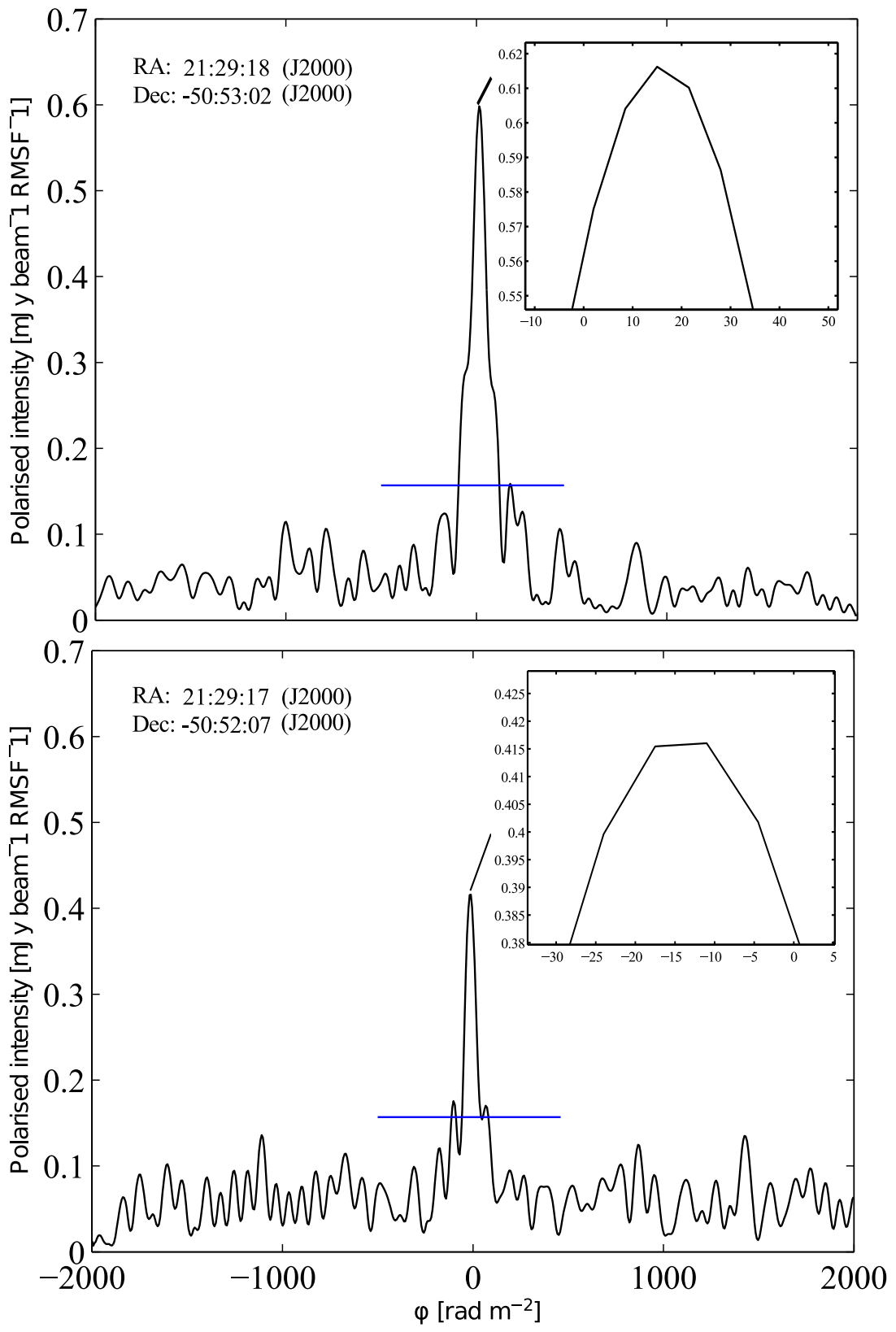

Figure 8.5: $\mathrm{F}(\phi)$ spectrum in two pixels of the HT galaxy. Top: The polarised intensity spectrum of a random pixel in the left jet. Bottom: The polarised intensity spectrum of a random pixel in the right lobe. The blue line represents the detection threshold in our RM cube. 


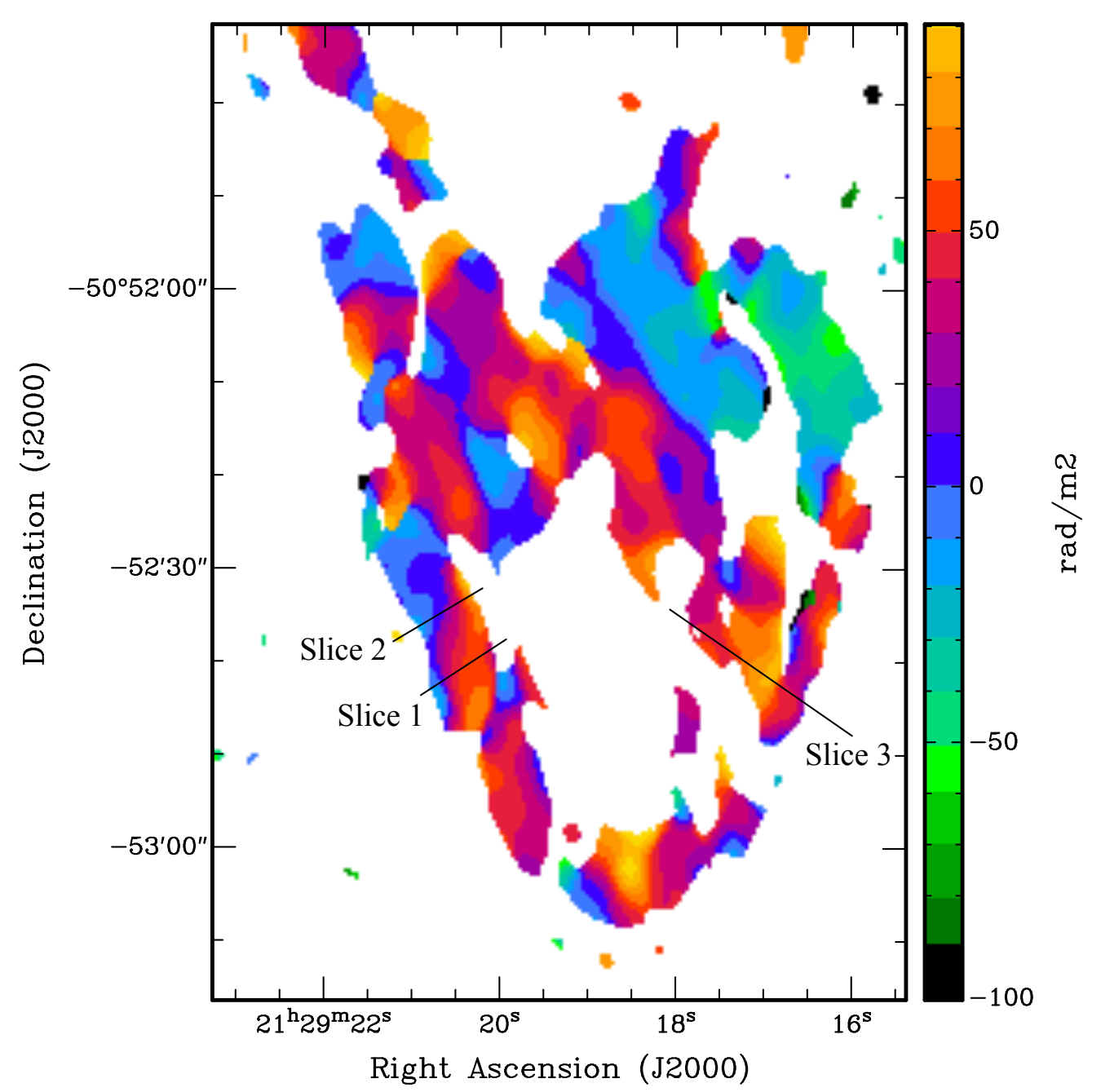

Figure 8.6: RM map of the HT galaxy in A3771. A dominant region with negative RM values in the right lobe with a sharp boundary is evident. The masked pixels are identical to the masked pixels in the PA image. The resolution of the RM map is $9^{\prime \prime} \times 6^{\prime \prime}$.

The HT galaxy under study has a Galactic latitude of -45.5, thus it is located far from the Galactic plane. As a result, the contribution of the Galactic magnetic field in the measured RMs is expected to be small. The Galactic rotation measure along the LOS of the HT galaxy was estimated as $-4.18 \mathrm{rad} / \mathrm{m}^{2}$ from the work presented by JohnstonHollitt et al. (2004b). The rest-frame RM values were calculated using the relation $R M_{o b s}=R M_{0} /(1+\mathrm{z})^{2}$. The rest-frame RM values slightly vary from the observed $\mathrm{RM}$ values in AGNs at low redshifts, therefore, this transformation is not vital however for the AGNs at higher redshifts this correction is highly suggested. This treatment allows us to compare the RM values of AGNs at different redshifts. Since our detected HT galaxy has a low redshift, the observed values are very close to the rest-frame values.

The initial RM map was created by locating the peak of the polarisation intensity in the re-constructed polarisation profile for each pixel. Then the Galactic contribution is subtracted from the derived RM values. We have investigated the constructed polarised 
intensity versus the Faraday depth in different pixels and it was easily realised that the Faraday depth has a single component at the detection limit. As a result, the Faraday depth can be thought as the Faraday rotation measure of the source. Figure 8.5, shows the $\mathrm{F}(\phi)$ spectrum in two random pixels located in the left jet and in the right lobe in the top and bottom plots, respectively. We have defined the detection limit of $5 \times \sigma_{p}$ where $\sigma_{p}=31 \mu \mathrm{Jybeam}^{-1} \mathrm{RMSF}^{-1}$ in $-500<\phi<500 \mathrm{rad} / \mathrm{m}^{2}$. Thus, the detection limit corresponds to the polarised intensity $157 \mu \mathrm{Jy} \mathrm{beam}^{-1} \mathrm{RMSF}^{-1}$ in the RM cube. The combination of the several pieces of evidence such as the shape $F(\phi)$ spectrum, shape of the RMTF and the detection limit in the RM cube lead to a conclusion that it is highly likely that the Faraday depth is a single component. Next we consider the distribution of peak RMs in all pixels in the source. In Figures 8.6 and 8.7 we show the map of the peak RMs and the histogram of their values, respectively.

Figure 8.6 shows a distinct and sharp boundary between the regions with positive and negative RM values in the right lobe. However, Figure 8.7 exhibits two peaks where one of the peaks occurs at negative RM values. This peak apparently corresponds to the $\mathrm{RM}$ values in the right lobe. We note that according to Figure8.1, the jet collimation is entirely lost in the right lobe.

Such features have been previously seen in the RM maps of radio galaxies in the literature. For instance, Guidetti et al. (2012) observed a radio source (B2 0755+37) with the Very Large Array (VLA) at three frequencies and studied the RM of the source in great details. They found that the derived RM map of E lobe had a complex RM structure. They reported a bimodal behaviour in the RM histogram of the observed radio galaxy, however, both of the peaks have been occurred at positive RM values. They find a peculiar feature in few ten kpc scales in East lobe in the radio galaxy. They report that the observed RM strip had the RM values close to zero in E lobe (see Figures 7 and 8 in Guidetti et al., 2012). Observations of such anomalies in our work and the work presented by Guidetti et al. (2012) are a mystery.

\section{§8.6. Magnetic Field Direction in SJ212919-505226}

As mentioned earlier in this chapter, the HT galaxy SJ212919-505226 was a great laboratory to study the magnetic field direction and structure in the radio galaxies. We have presented the initial magnetic field direction of the HT galaxy in Figure8.8 at 1.4 and $2.4 \mathrm{GHz}$ in the top and bottom plots, respectively. Intriguingly, we find that the direction of the magnetic field is predominantly perpendicular to the jet axes. This evidence is more pronounced in the left jet and this finding is approximately consistent in both frequencies. In the following section, we discuss the plausible explanation for the observed traverse magnetic field in HT galaxy, SJ212919-505226. 


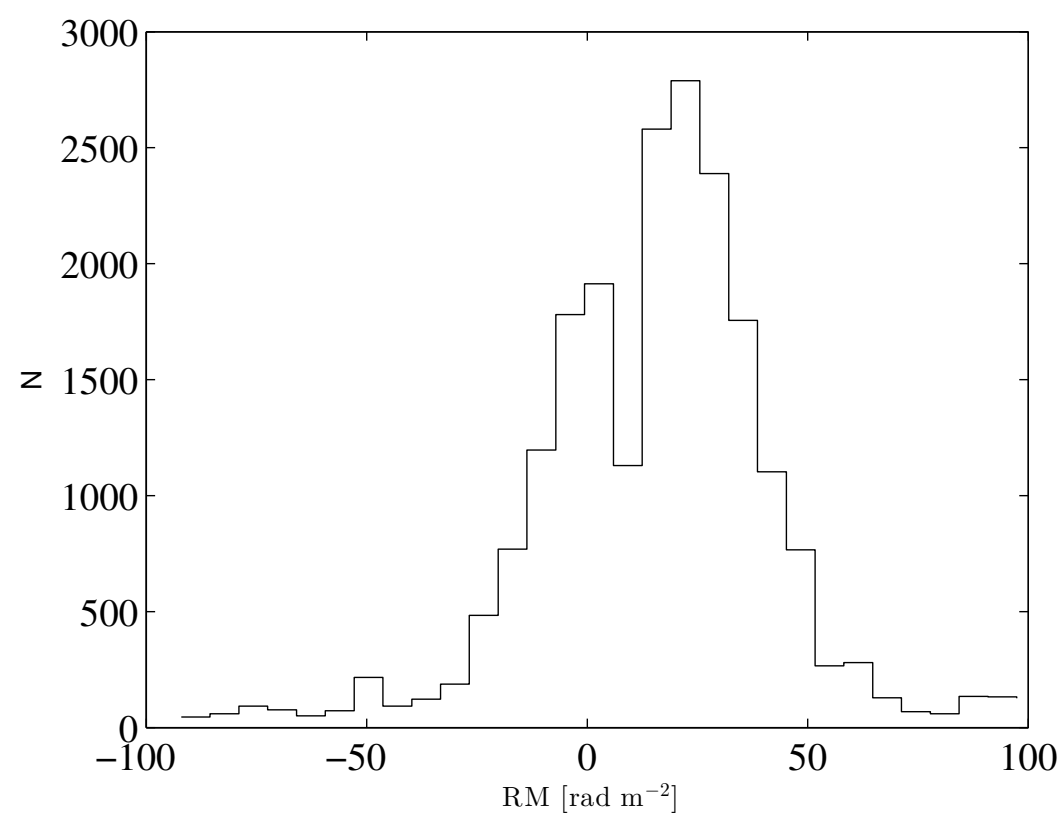

Figure 8.7: Histogram of the rest frame Galactic corrected RM values of the HT galaxy. The histogram shows two peaks which one of them occurs at negative RM values.

\subsection{Discussion}

Due to the small number of helical magnetic field detections, their origin and observational implications are not clear. However, one of the major observational properties of helical fields was proposed decades ago when Blandford (1993) pointed out that in the case that AGN has a helical magnetic field along the jets, the RM map should have a special signature. The toroidal component of the magnetic field induces a transverse gradient in the observed RM map across the jets. To be certain of a theoretical field, this trend should occur in multiple locations along the jets as locally induced RM gradient can also happen across the jets as a result of either the change of the density of the plasma or the magnetic field direction in small scales. Later on, Gabuzda et al. (2004) pointed out that the observed helical magnetic field structure can be heavily modified as a function of viewing angle. They argued that if the toroidal component is predominantly along the jets and the longitudinal component of the B field is barely observed along the edges, this can be interpreted such that the jets are "side-on" in the jet's frame. They stressed that in the case of a "side-on" view of a right-handed (RH) helical B-field, the polarisation intensity should be identical in both jets and a transverse RM gradient with sign change with values close to zero are expected to be observed across the jets. It should be noted that the radiation emitted at $90^{\circ}$ to the jet axis is observed at the angle $1 / \gamma$ at the observer's frame where $\gamma$ is the Lorentz factor of the jet flow. If the jets are observed at a different angle (not $90^{\circ}$ to the jet axis), a combination of the toroidal and longitudinal components can be observed where the toroidal component is dominant 

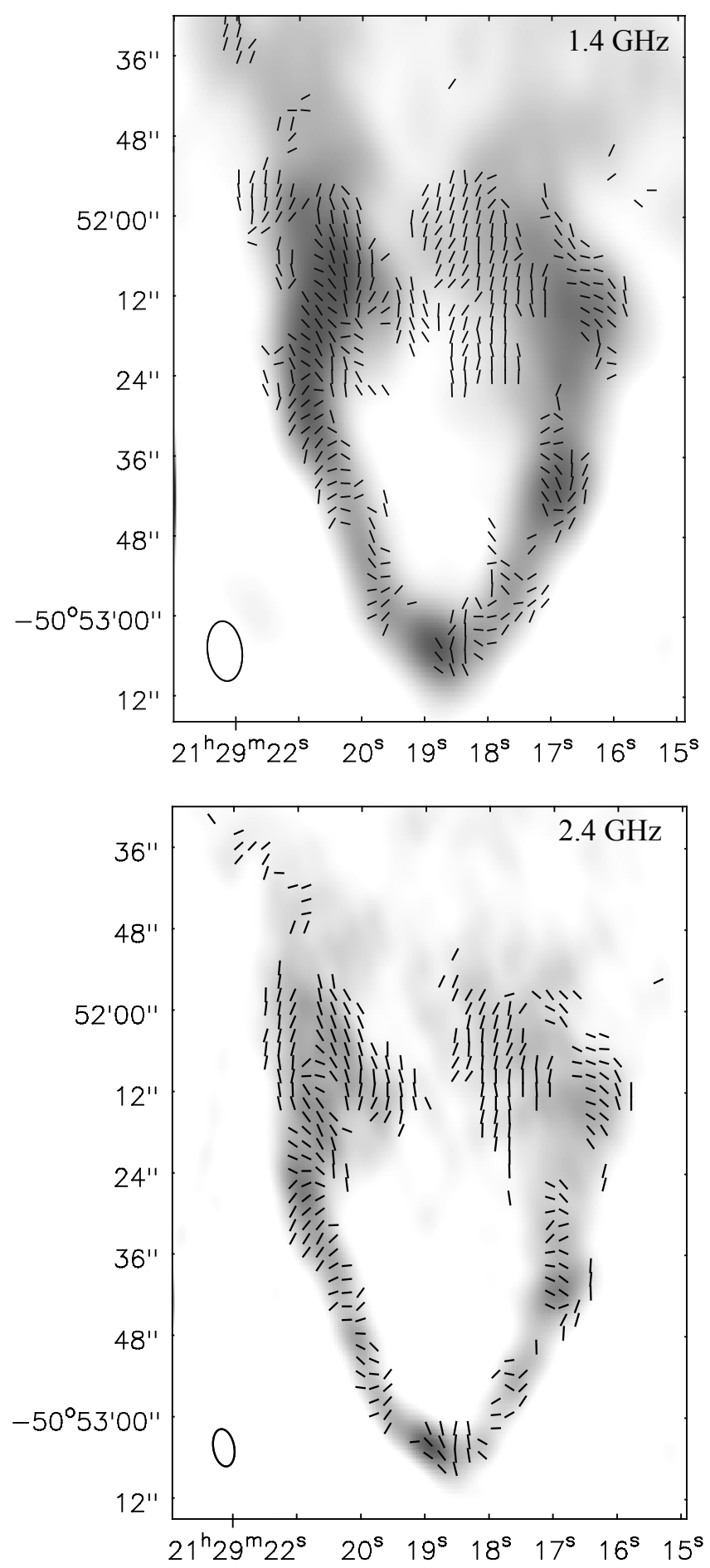

Figure 8.8: Magnetic field orientation over the $\mathrm{HT}$ galaxy at $1.4 \mathrm{GHz}$ and $2.4 \mathrm{GHz}$ are presented in top and bottom plots, respectively. The magnetic field direction is perpendicular to the jets. 

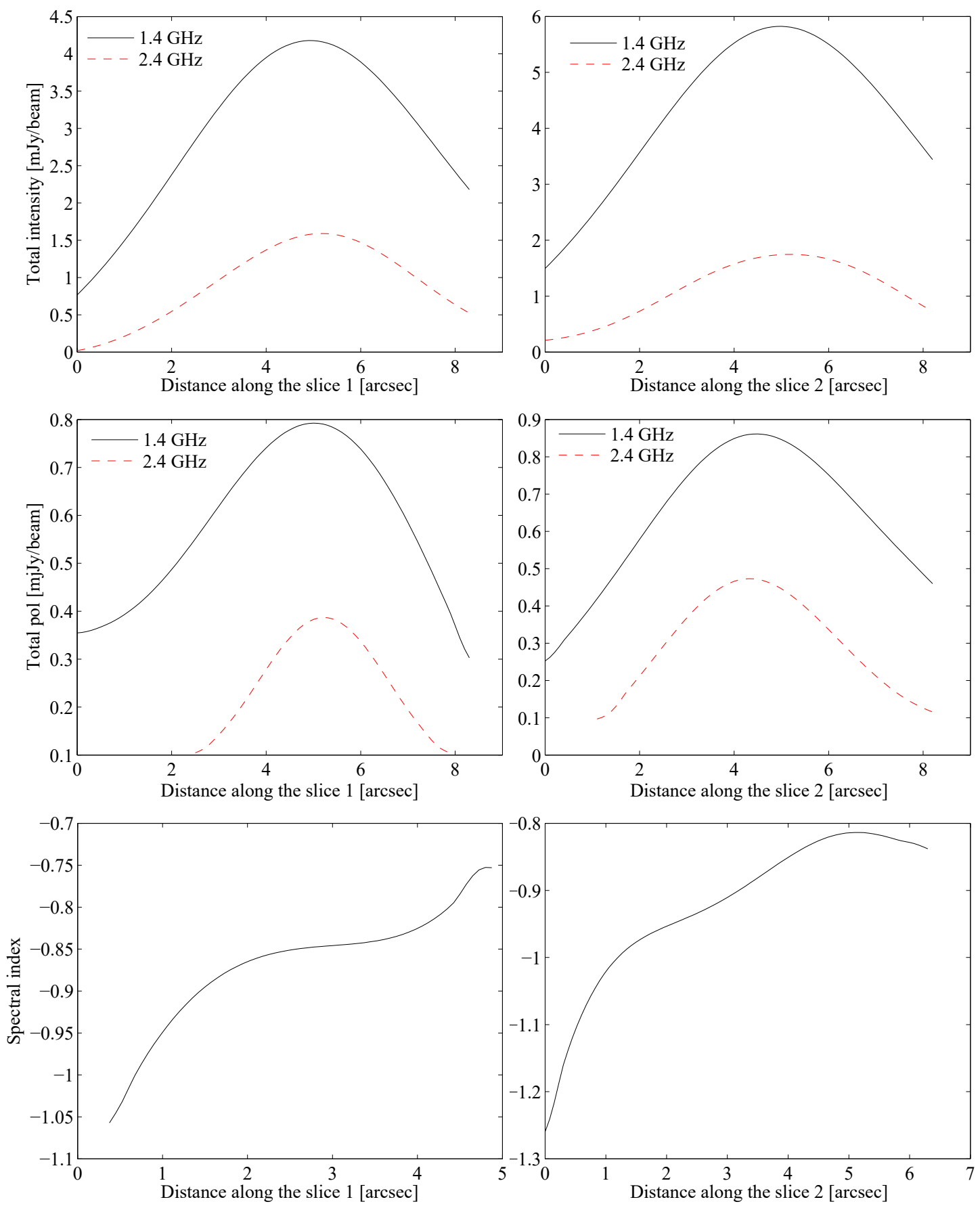

Figure 8.9: Total intensity profile along the slices 1 and 2 are shown in the two top plots. linearly polarised intensity along the slices 1 and 2 (middle plots) and spectral index are demonstrated in the middle and bottom panels, respectively. The observation at $1.4 \mathrm{GHz}$ and $2.4 \mathrm{GHz}$ are shown in black and red line, respectively. 

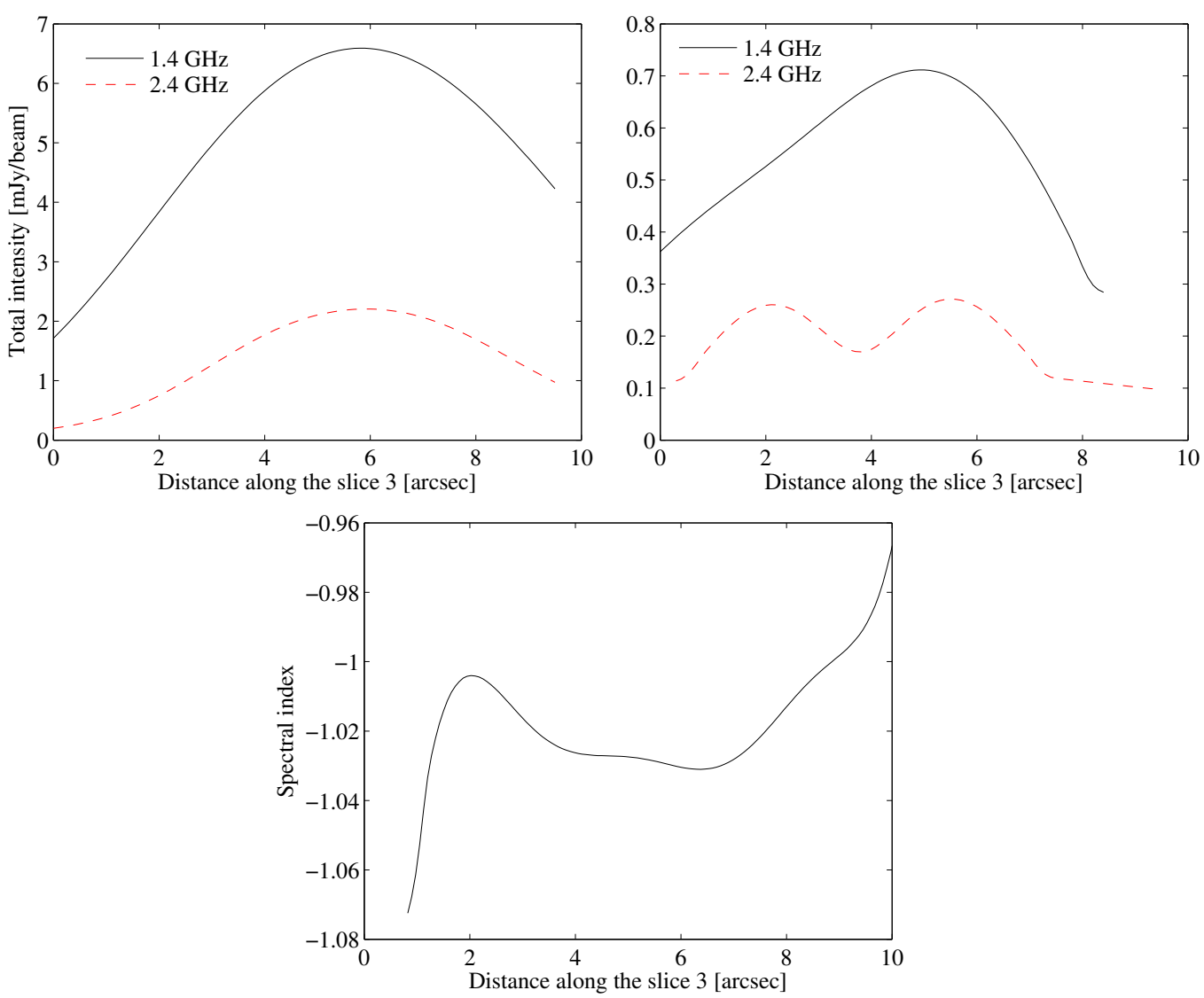

Figure 8.10: Total intensity, polarisation and spectral index profiles along the slice 3. This plot has the same descriptions given in Figure 8.9.

in the centre of the jets and the longitudinal components plays a significant role at the locations close to the edges of the jets.

The validity of the detection of such gradients across the jets have been under debate because only a small number of radio observations had enough resolution and sensitivity to resolve the jets. However, with the growing number of very long baseline interferometry (VLBI) observations and more sensitive broadband polarimetry in recent years, new observed transverse RM gradients are believed to be highly accurate.

Although detection of RM gradients is very hard, many projects have been carried out recently to study the RM structures of radio galaxies, therefore number of observed RM gradients has grown dramatically in recent years. Hovatta et al. (2012b) studied the RM maps of a sample of 191 extragalactic radio jets in the MOHAVE project. They carried out multi-epoch VLBA observations at four different frequencies from 4 to 8 GHz. They found evidence of transverse RM gradient in four of the observed radio sources and in one of them the sign reversal was also observed (1226+023). Later on, Gabuzda et al. (2015) observed 15 AGNs in frequency range 4.6-15.4 GHz and have reported six new transverse RM detections alongside the confirmation of seven previous detections. 
The theoretical studies of helical fields have not been progressed noticeably until recently that Clausen-Brown et al. (2011) studied the imprints of the helical magnetic field in the total brightness, polarisation and spectral index profiles across the jets. They argued that the Stokes I profile should exhibit skewness and the tail should be evident on the same side of the profile where the polarisation has lower values and the spectral index is steeper. The fractional polarisation should also increase towards the edges in the jets. However, observing such skewed profiles is highly dependent on the viewing angle and the Lorentz factor of the jet flow. Thus, the detection of skewed profiles is not quite straightforward.

Several parameters such as low $\mathrm{S} / \mathrm{N}$ ratio and higher errors can significantly modify the polarisation and spectral index profiles. On the other hand, tracing the signatures of skewness in the observable profiles is heavily dependent on the beam size as asymmetrical features can be smoothed out by larger beams.

It should be noted that the majority of the asymmetric profiles are observed in the VLBI observations which are only able to probe the spine of the jet in parsec scales. Thus, it is not well understood that to what extent, the proposed correlations between the observable profiles hold. On the other side, other parameters (except the beam size) which can effectively distort or smooth the possible asymmetries in arcsec observations are barely known.

Soon after the advancements in theoretical perspective, Hovatta et al. (2012b) investigated the total intensity, polarisation and spectral index profiles along one slice in the source 3C 273 and found out that all the aforementioned profiles display the suggested correlation claimed by Clausen-Brown et al. (2011). O'Sullivan and Gabuzda (2008) have studied six "blazars" (a sub-class of radio galaxies) at multiple frequencies with the American Very Long Baseline Array (VLBA) and found one RM gradient across the source $0954+658$. They examined the structure of the total and polarised intensity and the spectral index and found the same trend as it is suggested by Clausen-Brown et al. (2011).

We have chosen three slices across the jets. The slices were placed at the locations where the RM transverse gradient was more robust, the polarisation had better $\mathrm{S} / \mathrm{N}$ ratio and the projected electric vector position angle (EVPA) were clearly parallel to the jet axis. In Figure 8.9 and 8.10 , the profiles along the three slices in total intensity, polarisation and spectral index are shown. The skewness in the intensity profiles across three slices is evident and the steeper spectral indices are also observed at the same side where the tails in the total intensity profiles are observed. However, the decrease in the polarisation profile at the location of total intensity tail is not robust.

The spectral index along slices 1 and 3 have an offset from zero. This is because the pixel values were masked due to having higher errors. The offset from zero also 
occurred in the polarisation profiles at the location of two slices at $2.4 \mathrm{GHz}$. This is due to the fact that the pixels at the beginning of the slices had the polarisation intensity less than the $3 \sigma_{p o l}$ and were masked.

Although the proposed correlations in the total intensity, polarisation and spectral index are not robust in all locations along the jets, the combination of observed skewed profiles of the observables and the occurrence of the transverse RM gradient with the sign change at multiple locations across the jets and lobes suggest that the observed transverse magnetic field along the jets might be the toroidal component of a right-hand helical magnetic field. On the other hand, the increase of the fractional polarisation towards the outermost regions of the jets could suggest that a helical magnetic field is present in the HT jets, however it is known that at the edges of the source the results are less robust. The aforementioned evidence requires confirmation with on-axis polarimetric observations at higher frequencies.

\section{§8.8. Summary}

We have serendipitously detected an HT galaxy in A3771. This HT galaxy is likely a cluster member and is located at the SW of the cluster. We have used the CASA CLEANing procedure to exploit the virtue of the total two $\mathrm{GHz}$ bandwidth offered in CABB mode to create the spectral index and its error maps. We used nterms $=2$ (two Taylor expansion terms) in order to CLEAN the image. We also have used the multiscale CLEANing alongside to avoid potential problems induced by the point-source CLEANing method.

We have used the RM synthesis method to derive the RM cube of the HT galaxy using the entire band. The final Q and U cubes had 1506 channels. The RMTF had the FWHM of $65.7 \mathrm{rad} / \mathrm{m}^{2}$ and our RM cube was sensitive to Faraday thick sources. The reconstructed polarisation profile for different pixels showed that the Faraday dispersion function had a single component compared to the RMTF. We then derived the Galactic corrected RM map of the HT galaxy and the initial EVPAs were corrected for the RM for each pixel.

Intriguingly, the EVPAs were pre-dominantly parallel to the jet axis especially in the left jet where the signal-to-noise ratio was sufficient. We also have created the total intensity, polarisation and the spectral index profiles along the transverse slices to verify possible skewness in the observable profiles.

The observed profiles and the transverse RM gradient across the jet with the sign change in multiple locations could be indicative of a toroidal component of a helical magnetic field along the jets. Unfortunately, the signal-to-noise ratio in the right jet was not sufficient to recover the polarised emission. The transverse magnetic field direction 
is also evident in the lobes. The RM values in the right lobe were predominantly negative in a distinct region with an abrupt border in the middle of the right lobe. The nature of the existence of a region with predominant negative RM values was not clear.

This is a unique piece of work since; 1) this is the first evidence of the existence of toroidal component of a helical magnetic field in kpc-scale jets, 2) broadband polarimetry, spectral index analysis and RM synthesis of well-resolved jets were carried out over $\mathrm{a} \sim 2 \mathrm{GHz}$ bandwidth.

We suggest that a follow-up on-axis observation with the ATCA in any $6 \mathrm{~km}$ array configuration at higher frequencies ( 5 and $9 \mathrm{GHz}$ ) might be useful to probe the internal structures of the magnetic field. The on-axis observation diminishes the errors induced by the off-axis leakages in the polarimetry. 


\section{§. The Outlook}

\subsection{Future Radio Halo Surveys}

As discussed earlier in chapters 2 and 4, bias in selecting either disturbed or X-ray luminous galaxy clusters or even the combination of both is commonly seen in recent radio halo detections. In addition, as mentioned in chapter 4, apparently, radio observations of X-ray under-luminous clusters require more sensitivity to discover possible halos. Therefore, we can not assert whether the lack of radio halo observations in relaxed clusters are due to insufficient sensitivity of current radio telescopes or it is an actual phenomenon. As we showed in chapter 4, the observed bi-modality is not strong when the low-mass halo clusters are included in the analysis. Besides, our analysis showed that the confirmation of the bi-modality in low-mass systems requires radio observations with the unprecedented sensitivity to be able to detect radio halos with the predicted power $\left(\mathrm{P}_{1.4}<10^{23} \mathrm{~W} / \mathrm{Hz}\right)$.

There is a growing debate about the validity of the observed correlation between the thermal and non-thermal components of the halo clusters. The proposed correlations are claimed as firm evidence that confirms that merging is the key parameter in radio halo generation. However, the growing number of non-detections in disturbed clusters has raised many questions about the validity of this argument. The halo non-detections in disturbed clusters suggest that the cluster mass is possibly another major parameter in radio halo generation. The cluster mass estimations is not straightforward and it is believed that the integrated SZ signal of clusters is a better mass proxy.

As Sommer and Basu, 2014 mentioned, the artificial boost of the X-ray emission is noticeably higher than the SZ signal boost during and after the cluster merging. Therefore, it is believed that the mass derived from the integrated SZ signal is more reflective of the mass of the system in the relaxed phase. To verify the role of mass in halo generation, designing a mass selected cluster sample is vital. The South Pole Telescope (SPT) cluster survey is an ideal sample which can be used to design a mass selected sub-sample of clusters for the halo surveys. The SPT-SZ cluster survey has covered $\sim 2500 \mathrm{deg}^{2}$ of the Southern sky and found 465 massive clusters. The combination of the Planck DR2 and SPT-SZ cluster surveys can be used to design a mass-limited cluster sample, however, because only massive clusters are observed in both surveys, halo survey based on this selection is extremely biased towards massive systems. The upgrated SPT-3G commences surveying $2500 \mathrm{deg}^{2}$ of the Southern sky in the near future at a level that is 10 times deeper than the SPT-SZ survey. It will contain more high-redshift 


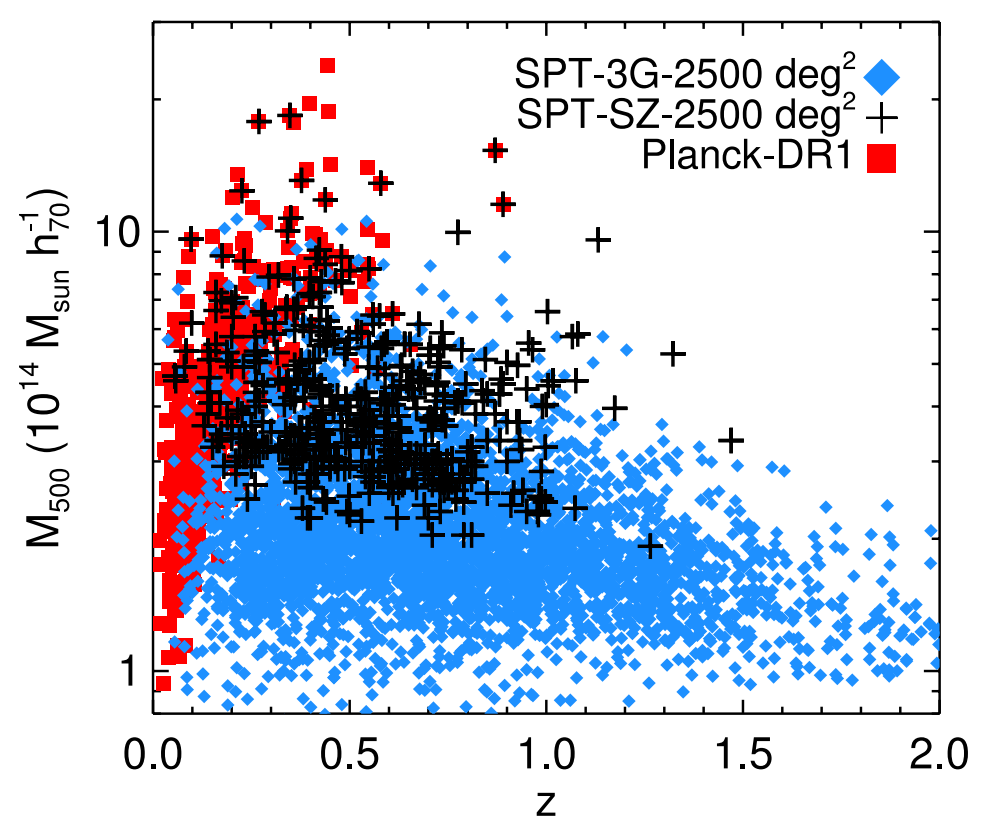

Figure 9.1: Comparison between current and future SZ cluster surveys. The image is adapted from the talk presented by B. Benson at the CARMA science symposium in 2013.

and low-mass clusters and thus this survey is ideal to select cluster sample for homogeneous halo surveys. In Figure 9.1, the comparison between current and near future SZ cluster surveys is shown.

From the radio observation perspective, the combination of ASKAP and MWA II can be used for the halo detection in the mass-selected clusters. Future radio surveys with telescopes such as ASKAP and SKA-mid with the unprecedented sensitivities between 2 to $10 \mu \mathrm{Jy}$ can significantly limit our challenges in diffuse radio emission detections in galaxy clusters. Blind radio surveys such as the evolutionary map of the Universe (EMU) with the Australian Square Kilometre Array Pathfinder (ASKAP) at $1.4 \mathrm{GHz}$ could detect more radio halos. The EMU survey is designed to be a full Southern sky radio survey with a sensitivity of $10 \mu \mathrm{Jy}$ which is 40 times better than the NVSS.

In addition to difficulties finding radio halos with moderate steep spectra, there is a large number of USS halos awaiting discovery according to the currently preferable model of halo generation. Therefore, low frequency observations in the frequency range 50-200 MHz are vital to discover the USS halos. Unfortunately, current low frequency interferometers such as the MWA do not have sufficient angular resolution to disentangle the radio halos from the other radio sources. Nonetheless, the upcoming MWA II is specifically designed to provide better resolution for various scientific goals and could be used for halo observations. Meanwhile, the upcoming gamma ray telescopes such as The Cherenkov Telescope Array (CTA) will enhance our knowledge about the cosmic ray content of the galaxy clusters and allows us to gain better understanding of the underlying physics involved in the radio halo generation. 



\section{Bibliography}

Abazajian, K. N., Adelman-McCarthy, J. K., Agüeros, M. A., Allam, S. S., Allende Prieto, C., An, D., Anderson, K. S. J., Anderson, S. F., Annis, J., Bahcall, N. A., and et al. (2009). The Seventh Data Release of the Sloan Digital Sky Survey. apjs, $182: 543-558$.

Abdo, A. A., Ackermann, M., Ajello, M., and others. (2009). Fermi Large Area Telescope Measurements of the Diffuse Gamma-Ray Emission at Intermediate Galactic Latitudes. Physical Review Letters, 103(25):251101.

Abell, G. O., Corwin, Jr., H. G., and Olowin, R. P. (1989). A catalog of rich clusters of galaxies. apjs, 70:1-138.

Akritas, M. G. and Bershady, M. A. (1996). Linear Regression for Astronomical Data with Measurement Errors and Intrinsic Scatter. apj, 470:706.

Arnaud, M., Pratt, G. W., Piffaretti, R., Böhringer, H., Croston, J. H., and Pointecouteau, E. (2010). The universal galaxy cluster pressure profile from a representative sample of nearby systems (REXCESS) and the $\mathrm{Y}_{S Z}-\mathrm{M}_{500}$ relation. aap, 517:A92.

Ashman, K. M., Bird, C. M., and Zepf, S. E. (1994). Detecting bimodality in astronomical datasets. $a j, 108: 2348-2361$.

Bacchi, M., Feretti, L., Giovannini, G., and Govoni, F. (2003). Deep images of cluster radio halos. aap, 400:465-476.

Bagchi, J., Enßlin, T. A., Miniati, F., Stalin, C. S., and others. (2002). Evidence for shock acceleration and intergalactic magnetic fields in a large-scale filament of galaxies $\mathrm{ZwCl} 2341.1+0000$. na, 7:249-277.

Basden, A. G., Evans, C. J., and Morris, T. J. (2014). Wide-field adaptive optics performance in cosmological deep fields for multi-object spectroscopy with the European Extremely Large Telescope. mnras, 445:4008-4014.

Basu, K. (2012a). A Sunyaev-Zel'dovich take on cluster radio halos - I. Global scaling and bi-modality using Planck data. mnras, 421:L112-L116.

Basu, K. (2012b). Some results on the radio-SZ correlation for galaxy cluster radio halos. ArXiv e-prints. 
Beck, R. and Krause, M. (2005). Revised equipartition and minimum energy formula for magnetic field strength estimates from radio synchrotron observations. Astronomische Nachrichten, 326:414-427.

Beers, T. C., Flynn, K., and Gebhardt, K. (1990). Measures of location and scale for velocities in clusters of galaxies - A robust approach. Astronomical Journal, 100:3246.

Begelman, M. C., Rees, M. J., and Blandford, R. D. (1979). A twin-jet model for radio trails. nat, 279:770-773.

Bicknell, G. V. (1995). Relativistic Jets and the Fanaroff-Riley Classification of Radio Galaxies. apjs, 101:29.

Blandford, R. (1993). Acceleration and collimation mechanisms in jets. In Astrophysics and Space Science Library, volume 103 of Astrophysics and Space Science Library, pages 15-33.

Blanton, E. L., Gregg, M. D., Helfand, D. J., Becker, R. H., and White, R. L. (2004). Uncovering High-z Clusters Using Wide-angle Tailed Radio Sources. Clusters of Galaxies: Probes of Cosmological Structure and Galaxy Evolution, page 4.

Blasi, P. and Colafrancesco, S. (1999). Cosmic rays, radio halos and nonthermal X-ray emission in clusters of galaxies. Astroparticle Physics, 12:169-183.

Blasi, P., Gabici, S., and Brunetti, G. (2007). Gamma Rays from Clusters of Galaxies. International Journal of Modern Physics A, 22:681-706.

Böhringer, H., Pratt, G. W., Arnaud, M., and others. (2010). Substructure of the galaxy clusters in the REXCESS sample: observed statistics and comparison to numerical simulations. aap, 514:A32.

Böhringer, H., Schuecker, P., Guzzo, L., and others. (2004). The ROSAT-ESO Flux Limited X-ray (REFLEX) Galaxy cluster survey. V. The cluster catalogue. aap, 425:367-383.

Böhringer, H., Schuecker, P., Pratt, and others. (2007). The representative XMMNewton cluster structure survey (REXCESS) of an X-ray luminosity selected galaxy cluster sample. aap, 469:363-377.

Bonafede, A., Bruggen, M., van Weeren, R., and others. (2012). Discovery of radio halos and double relics in distant MACS galaxy clusters: clues to the efficiency of particle acceleration. mnras, 426:40-56. 
Bonafede, A., Feretti, L., Giovannini, G., Govoni, F., Murgia, M., Taylor, G. B., Ebeling, H., Allen, S., Gentile, G., and Pihlström, Y. (2009a). Revealing the magnetic field in a distant galaxy cluster: discovery of the complex radio emission from MACS J0717.5 +3745. aap, 503:707-720.

Bonafede, A., Feretti, L., Murgia, M., Govoni, F., Giovannini, G., Dallacasa, D., Dolag, K., and Taylor, G. B. (2010). The Coma cluster magnetic field from Faraday rotation measures. aap, 513:A30.

Bonafede, A., Giovannini, G., Feretti, L., Govoni, F., and Murgia, M. (2009b). Double relics in Abell 2345 and Abell 1240. Spectral index and polarization analysis. aap, 494:429-442.

Bonafede, A., Govoni, F., Feretti, L., Murgia, M., Giovannini, G., and Brüggen, M. (2011). Fractional polarization as a probe of magnetic fields in the intra-cluster medium. aap, 530:A24.

Bonafede, A., Intema, H. T., Brüggen, M., Girardi, M., Nonino, M., Kantharia, N., van Weeren, R. J., and Röttgering, H. J. A. (2014). Evidence for Particle Reacceleration in the Radio Relic in the Galaxy Cluster PLCKG287.0+32.9. apj, 785:1.

Bonafede, A., Intema, H. T., Brüggen, M., and others. (2015). Radio halos in SZselected clusters of galaxies: the making of a halo? ArXiv e-prints.

Bravi, L., Gitti, M., and Brunetti, G. (2015). Do radio mini-halos and gas heating in cool-core clusters have a common origin? ArXiv e-prints.

Brentjens, M. A. and de Bruyn, A. G. (2005). Faraday rotation measure synthesis. aap, 441:1217-1228.

Bridle, A. H. and Fomalont, E. B. (1976). Complex radio emission from the X-ray cluster Abell 2256. aap, 52:107-113.

Brown, S., Emerick, A., Rudnick, L., and Brunetti, G. (2011). Probing the Off-state of Cluster Giant Radio Halos. apjl, 740:L28.

Brown, S. and Rudnick, L. (2009). Diffuse Cluster-Like Radio Emission in Poor Environments. aj, 137:3158-3171.

Brunetti, G., Blasi, P., Reimer, O., Rudnick, L., Bonafede, A., and Brown, S. (2012). Probing the origin of giant radio halos through radio and $\gamma$-ray data: the case of the Coma cluster. mnras, 426:956-968. 
Brunetti, G., Cassano, R., Dolag, K., and Setti, G. (2009). On the evolution of giant radio halos and their connection with cluster mergers. aap, 507:661-669.

Brunetti, G. and Jones, T. W. (2014). Cosmic Rays in Galaxy Clusters and Their Nonthermal Emission. International Journal of Modern Physics D, 23:30007.

Brunetti, G., Setti, G., Feretti, L., and Giovannini, G. (2001). Particle reacceleration in the Coma cluster: radio properties and hard X-ray emission. mnras, 320:365-378.

Brunetti, G., Venturi, T., Dallacasa, D., Cassano, R., Dolag, K., Giacintucci, S., and Setti, G. (2007). Cosmic Rays and Radio Halos in Galaxy Clusters: New Constraints from Radio Observations. apjl, 670:L5-L8.

Buote, D. A. (2001). On the Origin of Radio Halos in Galaxy Clusters. apjl, 553:L15L18.

Buote, D. A. and Tsai, J. C. (1995). Quantifying the Morphologies and Dynamical Evolution of Galaxy Clusters. I. The Method. apj, 452:522.

Burn, B. J. (1966). On the depolarization of discrete radio sources by Faraday dispersion. mnras, 133:67.

Burns, J. O. (1998). Stormy Weather in Galaxy Clusters. Science, 280:400.

Burns, J. O., Loken, C., Roettiger, K., Rizza, E., Bryan, G., Norman, M. L., Gómez, P., and Owen, F. N. (2002). Stormy weather and cluster radio galaxies. nar, 46:135140 .

Burns, J. O., Roettiger, K., Pinkney, J., Perley, R. A., Owen, F. N., and Voges, W. (1995). Evidence for an On-going Cluster/Group Merger in Abell 2255. apj, 446:583.

Carlson, E., Linden, T., Profumo, S., and Weniger, C. (2013). Clustering analysis of the morphology of the $130 \mathrm{GeV}$ gamma-ray feature. prd, 88(4):043006.

Cassano, R., Brunetti, G., and Setti, G. (2006). Statistics of giant radio halos from electron reacceleration models. mnras, 369:1577-1595.

Cassano, R., Brunetti, G., Setti, G., Govoni, F., and Dolag, K. (2007). New scaling relations in cluster radio halos and the re-acceleration model. mnras, 378:15651574.

Cassano, R., Brunetti, G., and Venturi, T. (2011). The Connection between Radio Halos and Cluster Mergers and the Statistical Properties of the Radio Halo Population. Journal of Astrophysics and Astronomy, 32:519-527. 
Cassano, R., Brunetti, G., Venturi, T., Setti, G., Dallacasa, D., Giacintucci, S., and Bardelli, S. (2008). Revised statistics of radio halos and the reacceleration model. aap, 480:687-697.

Cassano, R., Ettori, S., Brunetti, G., Giacintucci, S., Pratt, G. W., Venturi, T., Kale, R., Dolag, K., and Markevitch, M. (2013). Revisiting Scaling Relations for Giant Radio Halos in Galaxy Clusters. apj, 777:141.

Cassano, R., Ettori, S., Giacintucci, S., Brunetti, G., Markevitch, M., Venturi, T., and Gitti, M. (2010). On the Connection Between Giant Radio Halos and Cluster Mergers. apjl, 721:L82-L85.

Chon, G., Böhringer, H., and Smith, G. P. (2012). Statistics and implications of substructure detected in a representative sample of X-ray clusters. aap, 548:A59.

Clarke, T. E. (2004). Faraday Rotation Observations of Magnetic Fields in Galaxy Clusters. Journal of Korean Astronomical Society, 37:337-342.

Clarke, T. E. and Ensslin, T. (2006a). Mergers, relics and magnetic fields: The complex case of A2256. Astronomische Nachrichten, 327:553.

Clarke, T. E. and Ensslin, T. A. (2006b). Deep 1.4 GHz Very Large Array Observations of the Radio Halo and Relic in Abell 2256. aj, 131:2900-2912.

Clarke, T. E., Kronberg, P. P., and Böhringer, H. (2001). A New Radio-X-Ray Probe of Galaxy Cluster Magnetic Fields. apjl, 547:L111-L114.

Clausen-Brown, E., Lyutikov, M., and Kharb, P. (2011). Signatures of large-scale magnetic fields in active galactic nuclei jets: transverse asymmetries. mnras, 415:20812092.

Cohen, A. S. and Clarke, T. E. (2011). An Ultra-steep-spectrum Radio Relic in the Galaxy Cluster A2443. aj, 141:149.

Colless, M. (1999). First results from the 2dF Galaxy Redshift Survey. In Efstathiou, G. and et al., editors, Large-Scale Structure in the Universe, page 105.

Colless, M., Dalton, G., Maddox, S., Sutherland, W., and others. (2001). The 2dF Galaxy Redshift Survey: spectra and redshifts. mnras, 328:1039-1063.

Colless, M. and Dunn, A. M. (1996). Structure and Dynamics of the Coma Cluster. apj, 458:435.

Colless, M., Peterson, B. A., Jackson, and others. (2003). The 2dF Galaxy Redshift Survey: Final Data Release. ArXiv Astrophysics e-prints. 
Collins, C. A., Guzzo, L., Nichol, R. C., and Lumsden, S. L. (1995). The EdinburghDurham Southern Galaxy Catalogue - VII. The Edinburgh-Milano cluster redshift survey. mnras, 274:1071-1092.

Condon, J. J. (1992). Radio emission from normal galaxies. araa, 30:575-611.

Condon, J. J., Cotton, W. D., Greisen, E. W., Yin, Q. F., Perley, R. A., Taylor, G. B., and Broderick, J. J. (1998). The NRAO VLA Sky Survey. aj, 115:1693-1716.

Corwin, Jr., H. G. and Emerson, D. (1982). Optical spectra and redshifts for seventy-one galaxies. mnras, 200:621-644.

Croom, S. M., Smith, R. J., Boyle, B. J., Shanks, T., Loaring, N. S., Miller, L., and Lewis, I. J. (2001). The 2dF QSO Redshift Survey - V. The 10k catalogue. mnras, 322:L29-L36.

Dallacasa, D., Brunetti, G., Giacintucci, S., Cassano, R., Venturi, T., Macario, G., Kassim, N. E., Lane, W., and Setti, G. (2009). Deep 1.4 GHz Follow-up of the Steep Spectrum Radio Halo in A521. apj, 699:1288-1292.

de Gasperin, F., Intema, H. T., van Weeren, R. J., Dawson, W. A., Golovich, N., Wittman, D., Bonafede, A., and Brüggen, M. (2015). A powerful double radio relic system discovered in PSZ1 G108.18-11.53: evidence for a shock with nonuniform Mach number? mnras, 453:3483-3498.

Dehghan, S. and Johnston-Hollitt, M. (2014). Clusters, Groups, and Filaments in the Chandra Deep Field-South up to Redshift 1. aj, 147:52.

Dehghan, S., Johnston-Hollitt, M., Franzen, T. M. O., Norris, R. P., and Miller, N. A. (2014). Bent-tailed Radio Sources in the Australia Telescope Large Area Survey of the Chandra Deep Field South. aj, 148:75.

Deiss, B. M. (1997). A deprojection method to obtain the spectral index distribution in diffuse radio halos of clusters of galaxies. aap, 325:74-80.

Dennison, B. (1980). Formation of radio halos in clusters of galaxies from cosmic-ray protons. apjl, 239:L93-L96.

Diaferio, A. (1999). Mass estimation in the outer regions of galaxy clusters. mnras, 309:610-622.

Diaferio, A. (2009). Measuring the mass profile of galaxy clusters beyond their virial radius. ArXiv e-prints.

Diaferio, A. and Geller, M. J. (1997). Infall Regions of Galaxy Clusters. apj, 481:633. 
Dolag, K., Bartelmann, M., and Lesch, H. (1999). SPH simulations of magnetic fields in galaxy clusters. aap, 348:351-363.

Dolag, K., Bartelmann, M., and Lesch, H. (2002). Evolution and structure of magnetic fields in simulated galaxy clusters. aap, 387:383-395.

Dolag, K. and Stasyszyn, F. (2009). An MHD GADGET for cosmological simulations. mnras, 398:1678-1697.

Dolag, K., Vazza, F., Brunetti, G., and Tormen, G. (2005). Turbulent gas motions in galaxy cluster simulations: the role of smoothed particle hydrodynamics viscosity. mnras, 364:753-772.

Drury, L. O. (1983). An introduction to the theory of diffusive shock acceleration of energetic particles in tenuous plasmas. Reports on Progress in Physics, 46:9731027.

Dwarakanath, K. S., Malu, S., and Kale, R. (2011). Discovery of a Giant Radio Halo in a Massive Merging Cluster at $\mathrm{z}=0.443$. Journal of Astrophysics and Astronomy, 32:529-532.

Ebeling, H., Edge, A. C., Bohringer, H., and others. (1998). The ROSAT Brightest Cluster Sample - I. The compilation of the sample and the cluster $\log \mathrm{N}-\log \mathrm{S}$ distribution. mnras, 301:881-914.

Ensslin, T., Pfrommer, C., Miniati, F., and Subramanian, K. (2011). Cosmic ray transport in galaxy clusters: implications for radio halos, gamma-ray signatures, and cool core heating. aap, 527:A99.

Ensslin, T. A., Biermann, P. L., Klein, U., and Kohle, S. (1998). Cluster radio relics as a tracer of shock waves of the large-scale structure formation. aap, 332:395-409.

Enßlin, T. A. and Gopal-Krishna (2001). Reviving fossil radio plasma in clusters of galaxies by adiabatic compression in environmental shock waves. aap, 366:26-34.

Ester, M., Kriegal, H. P., and Sander, J. (1996). A density-based algorithm for discovering clusters in large spatial databases with noise. Proc. 2nd Int. Conf. on Knowledge Discovery and Data Mining (KDD96). AAAI Press, Menlo Park, CA, pages 226-231.

Fanaroff, B. L. and Riley, J. M. (1974). The morphology of extragalactic radio sources of high and low luminosity. mnras, 167:31P-36P. 
Farnsworth, D., Rudnick, L., Brown, S., and Brunetti, G. (2013). Discovery of Megaparsec-scale, Low Surface Brightness Nonthermal Emission in Merging Galaxy Clusters Using the Green Bank Telescope. apj, 779:189.

Fasano, G., Marmo, C., Varela, J., D’Onofrio, M., Poggianti, B. M., Moles, M., Pignatelli, E., Bettoni, D., Kjærgaard, P., Rizzi, L., Couch, W. J., and Dressler, A. (2006). WINGS: a WIde-field Nearby Galaxy-cluster Survey. I. Optical imaging. aap, 445:805-817.

Feretti, L., Dallacasa, D., Govoni, F., Giovannini, G., Taylor, G. B., and Klein, U. (1999). The radio galaxies and the magnetic field in Abell 119. aap, 344:472-482.

Feretti, L., Fusco-Femiano, R., Giovannini, G., and Govoni, F. (2001). The giant radio halo in Abell 2163. aap, 373:106-112.

Feretti, L., Giovannini, G., and Böhringer, H. (1997). The radio and X-ray properties of Abell 2319. na, 2:501-515.

Feretti, L., Giovannini, G., Govoni, F., and Murgia, M. (2012). Clusters of galaxies: observational properties of the diffuse radio emission. aapr, 20:54.

Feretti, L., Orrù, E., Brunetti, G., Giovannini, G., Kassim, N., and Setti, G. (2004). Spectral index maps of the radio halos in Abell 665 and Abell 2163. aap, 423:111119.

Ferrari, C., Govoni, F., Schindler, S., Bykov, A. M., and Rephaeli, Y. (2008). Observations of Extended Radio Emission in Clusters. ssr, 134:93-118.

Fitchett, M. (1988). The statistical determination of substructure in clusters of galaxies. mnras, 230:161-181.

Fleenor, M. C., Rose, J. A., Christiansen, W. A., Johnston-Hollitt, M., Hunstead, R. W., Drinkwater, M. J., and Saunders, W. (2006). Redshifts and Velocity Dispersions of Galaxy Clusters in the Horologium-Reticulum Supercluster. aj, 131:1280-1287.

Frater, R. H., Brooks, J. W., and Whiteoak, J. B. (1992). The Australia Telescope Overview. Journal of Electrical and Electronics Engineering Australia, 12:103112.

Freeland, E., Cardoso, R. F., and Wilcots, E. (2008). Bent-Double Radio Sources as Probes of Intergalactic Gas. apj, 685:858-862.

Gabuzda, D. C., Knuettel, S., and Reardon, B. (2015). Transverse Faraday-rotation gradients across the jets of 15 active galactic nuclei. mnras, 450:2441-2450. 
Gabuzda, D. C., Murray, É., and Cronin, P. (2004). Helical magnetic fields associated with the relativistic jets of four BL Lac objects. mnras, 351:L89-L93.

Gaensler, B., Agudo, I., Akahori, T., and others. (2015). Broadband Polarimetry with the Square Kilometre Array: A Unique Astrophysical Probe. Advancing Astrophysics with the Square Kilometre Array (AASKA14), page 103.

Gardner, J. P. (2015). High-Redshift Galaxies with the James Webb Space Telescope. IAU General Assembly, 22:57143.

Giacintucci, S., Kale, R., Wik, D. R., Venturi, T., and Markevitch, M. (2013). Discovery of a Giant Radio Halo in a New Planck Galaxy Cluster PLCKG171.9-40.7. apj, 766:18.

Giacintucci, S., Markevitch, M., Brunetti, G., ZuHone, J. A., Venturi, T., Mazzotta, P., and Bourdin, H. (2014). Mapping the Particle Acceleration in the Cool Core of the Galaxy Cluster RX J1720.1+2638. apj, 795:73.

Giovannini, G., Bonafede, A., Feretti, L., Govoni, F., and Murgia, M. (2010). The diffuse radio filament in the merging system $\mathrm{ZwCl} 2341.1+0000$. aap, 511:L5.

Giovannini, G., Bonafede, A., Feretti, L., and others. (2009). Radio halos in nearby (z i 0.4 ) clusters of galaxies. aap, 507:1257-1270.

Giovannini, G. and Feretti, L. (2000). Halo and relic sources in clusters of galaxies. $n a$, 5:335-347.

Giovannini, G., Feretti, L., Venturi, T., Kim, K.-T., and Kronberg, P. P. (1993). The halo radio source Coma $\mathrm{C}$ and the origin of halo sources. apj, 406:399-406.

Goto, T., Okamura, S., Yagi, and others. (2003). Morphological Butcher-Oemler Effect in the SDSS "Cut and Enhance" Galaxy Cluster Catalog. pasj, 55:739-755.

Govoni, F., Enßlin, T. A., Feretti, L., and Giovannini, G. (2001). A comparison of radio and X-ray morphologies of four clusters of galaxies containing radio halos. aap, 369:441-449.

Govoni, F., Ferrari, C., Feretti, L., Vacca, V., Murgia, M., Giovannini, G., Perley, R., and Benoist, C. (2012). Detection of diffuse radio emission in the galaxy clusters A800, A910, A1550, and CL 1446+26. aap, 545:A74.

Govoni, F., Murgia, M., Feretti, L., Giovannini, G., Dallacasa, D., and Taylor, G. B. (2005). A2255: The first detection of filamentary polarized emission in a radio halo. aap, 430:L5-L8. 
Govoni, F., Murgia, M., Xu, H., Li, H., Norman, M. L., Feretti, L., Giovannini, G., and Vacca, V. (2013). Polarization of cluster radio halos with upcoming radio interferometers. aap, 554:A102.

Grazian, A., Omizzolo, A., Corbally, C., Cristiani, S., Haehnelt, M. G., and Vanzella, E. (2002). The Asiago-ESO/RASS QSO Survey. II. The Southern Sample. aj, 124:2955-2970.

Griffin, R. D., Dai, X., and Kochanek, C. S. (2014). New Limits on Gamma-Ray Emission from Galaxy Clusters. apjl, 795:L21.

Guidetti, D., Laing, R. A., Croston, J. H., Bridle, A. H., and Parma, P. (2012). The magnetized medium around the radio galaxy B2 0755+37: an interaction with the intragroup gas. mnras, 423:1335-1350.

Guidetti, D., Murgia, M., Govoni, F., Parma, P., Gregorini, L., de Ruiter, H. R., Cameron, R. A., and Fanti, R. (2008). The intracluster magnetic field power spectrum in Abell 2382. aap, 483:699-713.

Gunn, J. E. and Gott, III, J. R. (1972). On the Infall of Matter Into Clusters of Galaxies and Some Effects on Their Evolution. apj, 176:1.

Gursky, H. and Schwartz, D. A. (1977). Extragalactic X-ray sources. araa, 15:541-568.

Guzzo, L., Schuecker, P., Böhringer, H., and others. (2009). The REFLEX galaxy cluster survey. VIII. Spectroscopic observations and optical atlas,. aap, 499:357369.

Haarsma, D. B., Leisman, L., Bruch, S., and Donahue, M. (2009). Brightest Cluster Galaxies in REXCESS Clusters. In American Astronomical Society Meeting Abstracts 213, volume 41 of Bulletin of the American Astronomical Society, page 448.23 .

Haarsma, D. B., Leisman, L., Donahue, M., Bruch, S., Böhringer, H., Croston, J. H., Pratt, G. W., Voit, G. M., Arnaud, M., and Pierini, D. (2010). Brightest Cluster Galaxies and Core Gas Density in REXCESS Clusters. apj, 713:1037-1047.

Haines, C. P., Pereira, M. J., Sanderson, A. J. R., and others. (2012). LoCuSS: A Dynamical Analysis of X-Ray Active Galactic Nuclei in Local Clusters. apj, 754:97.

Hambly, N. C., Irwin, M. J., and MacGillivray, H. T. (2001a). The SuperCOSMOS Sky Survey - II. Image detection, parametrization, classification and photometry. mnras, 326:1295-1314. 
Hambly, N. C., MacGillivray, H. T., Read, and others. (2001b). The SuperCOSMOS Sky Survey - I. Introduction and description. mnras, 326:1279-1294.

Hardcastle, M. J., Worrall, D. M., Birkinshaw, M., Laing, R. A., and Bridle, A. H. (2002). A Chandra observation of the X-ray environment and jet of 3C 31. mnras, 334:182-192.

Harris, D. E. and Miley, G. K. (1978). Westerbork observations of five fields centered on Abell clusters of galaxies. aaps, 34:117-128.

Harris, D. E., Stern, C. P., Willis, A. G., and Dewdney, P. E. (1993). The megaparsec radio relic in supercluster, Rood No. 27. aj, 105:769-777.

Heald, G. H., Pizzo, R. F., Orrú, E., Breton, R. P., Carbone, D., Ferrari, C., Hardcastle, M. J., Jurusik, W., and others. (2015). The LOFAR Multifrequency Snapshot Sky Survey (MSSS) I. Survey description and first results. ArXiv e-prints.

Hennessy, G. S., Owen, F. N., and Eilek, J. A. (1989). Faraday rotation from Abell clusters of galaxies. apj, 347:144-151.

Hovatta, T., Lister, M. L., Aller, M. F., Aller, H. D., Homan, D. C., Kovalev, Y. Y., Pushkarev, A. B., and Savolainen, T. (2012a). Faraday rotation in the MOJAVE blazars: 3C 273 a case study. Journal of Physics Conference Series, 355(1):012008.

Hovatta, T., Lister, M. L., Aller, M. F., Aller, H. D., Homan, D. C., Kovalev, Y. Y., Pushkarev, A. B., and Savolainen, T. (2012b). MOJAVE: Monitoring of Jets in Active Galactic Nuclei with VLBA Experiments. VIII. Faraday Rotation in Parsecscale AGN Jets. aj, 144:105.

Huchra, J. P., Macri, L. M., Masters, K. L., Jarrett, T. H., Berlind, P., Calkins, M., Crook, A. C., Cutri, R., Erdoğdu, P., Falco, E., George, T., Hutcheson, C. M., Lahav, O., Mader, J., Mink, J. D., Martimbeau, N., Schneider, S., Skrutskie, M., Tokarz, S., and Westover, M. (2012). The 2MASS Redshift Survey-Description and Data Release. apjs, 199:26.

Jamrozy, M., Stawarz, Ł., Marchenko, V., Kuźmicz, A., Ostrowski, M., Cheung, C. C., and Sikora, M. (2014). Peculiar radio structures in the central regions of galaxy cluster Abell 585. mnras, 441:1260-1269.

Johnston-Hollitt, M. (2003). Detection of magnetic fields and diffuse radio emission in Abell 3667 and other rich southern clusters of galaxies. PhD thesis, University of Adelaide. 
Johnston-Hollitt, M. (2004). The Magnetic Field in A3667. In Reiprich, T., Kempner, J., and Soker, N., editors, The Riddle of Cooling Flows in Galaxies and Clusters of galaxies, page 51 .

Johnston-Hollitt, M., Clay, R. W., Ekers, R. D., Wieringa, M. H., and Hunstead, R. W. (2002). The Dual Radio Relics of A3667. In Pramesh Rao, A., Swarup, G., and Gopal-Krishna, editors, The Universe at Low Radio Frequencies, volume 199 of IAU Symposium, page 157.

Johnston-Hollitt, M. and Ekers, R. D. (2004). Faraday Rotation Measures through the Cores of Southern Galaxy Clusters. ArXiv Astrophysics e-prints.

Johnston-Hollitt, M., Fleenor, M., Rose, J., Christiansen, W., and Hunstead, R. W. (2004a). Radio imaging of head-tailed galaxies from an in-falling filament of the Horologium-Reticulum supercluster. In Diaferio, A., editor, IAU Colloq. 195: Outskirts of Galaxy Clusters: Intense Life in the Suburbs, pages 423-425.

Johnston-Hollitt, M., Govoni, F., Beck, R., and others. (2015). Using SKA Rotation Measures to Reveal the Mysteries of the Magnetised Universe. Advancing Astrophysics with the Square Kilometre Array (AASKA14), page 92.

Johnston-Hollitt, M., Hollitt, C. P., and Ekers, R. D. (2004b). Statistical Analysis of Extra-galactic Rotation Measures. In Uyaniker, B., Reich, W., and Wielebinski, R., editors, The Magnetized Interstellar Medium, pages 13-18.

Johnston-Hollitt, M., Hunstead, R. W., and Corbett, E. (2008). The optical morphology of A3667 re-examined. aap, 479:1-8.

Jones, D. H., Read, M. A., and others.. (2009). The 6dF Galaxy Survey: final redshift release (DR3) and southern large-scale structures. mnras, 399:683-698.

Kaiser, N. (1987). Clustering in real space and in redshift space. mnras, 227:1-21.

Kale, R. and Dwarakanath, K. S. (2010). Spectral Index Studies of the Diffuse Radio Emission in Abell 2256: Implications for Merger Activity. apj, 718:939-946.

Kale, R., Venturi, T., Giacintucci, and others. (2013). The Extended GMRT Radio Halo Survey. I. New upper limits on radio halos and mini-halos. aap, 557:A99.

Kale, R., Venturi, T., Giacintucci, S., and others. (2015). The Extended GMRT Radio Halo Survey. II. Further results and analysis of the full sample. aap, 579:A92.

Kassim, N. E., Clarke, T. E., Enßlin, T. A., Cohen, A. S., and Neumann, D. M. (2001). Low-Frequency VLA Observations of Abell 754: Evidence for a Cluster Radio Halo and Possible Radio Relics. apj, 559:785-790. 
Kennicutt, Jr., R. C. (1992). A spectrophotometric atlas of galaxies. apjs, 79:255-284.

Kim, K.-T. (1990). On the origin of the radio halo in the Coma cluster of galaxies. Nuovo Cimento B Serie, 105:845-856.

Kim, K.-T., Tribble, P. C., and Kronberg, P. P. (1991). Detection of excess rotation measure due to intracluster magnetic fields in clusters of galaxies. apj, 379:80-88.

Kinney, A. L., Calzetti, D., Bohlin, and others. (1996). Template Ultraviolet to NearInfrared Spectra of Star-forming Galaxies and Their Application to K-Corrections. apj, 467:38.

Ledlow, M. J. and Owen, F. N. (1996). 20 CM VLA Survey of Abell Clusters of Galaxies. VI. Radio/Optical Luminosity Functions. aj, 112:9.

Lee, k. L. (1979). Multivariate Tests for Clusters. J.Am.Stat.Assoc, 74:708714.

Liang, H., Hunstead, R. W., Birkinshaw, M., and Andreani, P. (2000). A Powerful Radio Halo in the Hottest Known Cluster of Galaxies 1E 0657-56. apj, 544:686-701.

Loveday, J. (1996). The APM Bright Galaxy Catalogue. mnras, 278:1025-1048.

Macario, G., Intema, H. T., Ferrari, C., and others. (2014). Discovery of large-scale diffuse radio emission and of a new galaxy cluster in the surroundings of MACS J0520.7-1328. aap, 565:A13.

Macario, G., Venturi, T., Dallacasa, D., Giacintucci, S., Brunetti, G., Cassano, R., Ishwara-Chandra, C. H., and Athreya, R. (2011). GMRT $150 \mathrm{MHz}$ follow up of diffuse steep spectrum radio emission in galaxy clusters. memsai, 82:557.

Machalski, J., Kozieł-Wierzbowska, D., Jamrozy, M., and Saikia, D. J. (2008). J14200545: The Radio Galaxy Larger than 3C 236. apj, 679:149-155.

Mann, A. W. and Ebeling, H. (2012). X-ray-optical classification of cluster mergers and the evolution of the cluster merger fraction. mnras, 420:2120-2138.

Mao, M. Y., Johnston-Hollitt, M., Stevens, J. B., and Wothers.poon, S. J. (2009). Headtail Galaxies: beacons of high-density regions in clusters. mnras, 392:1070-1079.

Mao, M. Y., Sharp, R., Saikia, D. J., Norris, R. P., Johnston-Hollitt, M., Middelberg, E., and Lovell, J. E. J. (2010). Wide-angle tail galaxies in ATLAS. mnras, 406:25782590.

Mauch, T., Murphy, T., Buttery, H. J., Curran, J., Hunstead, R. W., Piestrzynski, B., Robertson, J. G., and Sadler, E. M. (2003). SUMSS: a wide-field radio imaging survey of the southern sky - II. The source catalogue. mnras, 342:1117-1130. 
McKinney, J. C., Tchekhovskoy, A., Sadowski, A., and Narayan, R. (2014). Threedimensional general relativistic radiation magnetohydrodynamical simulation of super-Eddington accretion, using a new code HARMRAD with M1 closure. mnras, 441:3177-3208.

Mikellides, I. G., Tassis, K., and Yorke, H. W. (2011). 2D Magnetohydrodynamics simulations of induced plasma dynamics in the near-core region of a galaxy cluster. mnras, 410:2602-2616.

Miley, G. K., Perola, G. C., van der Kruit, P. C., and van der Laan, H. (1972). Active Galaxies with Radio Trails in Clusters. nat, 237:269-272.

Miniati, F., Ryu, D., Kang, H., and Jones, T. W. (2001). Cosmic-Ray Protons Accelerated at Cosmological Shocks and Their Impact on Groups and Clusters of Galaxies. apj, 559:59-69.

Mohr, J. J., Fabricant, D. G., and Geller, M. J. (1993). An X-ray method for detecting substructure in galaxy clusters - Application to Perseus, A2256, Centaurus, Coma, and Sersic 40/6. apj, 413:492-505.

Murgia, M., Govoni, F., Feretti, L., and Giovannini, G. (2010). A double radio halo in the close pair of galaxy clusters Abell 399 and Abell 401. aap, 509:A86.

Murgia, M., Govoni, F., Markevitch, M., Feretti, L., Giovannini, G., Taylor, G. B., and Carretti, E. (2009). Comparative analysis of the diffuse radio emission in the galaxy clusters A1835, A2029, and Ophiuchus. aap, 499:679-695.

Murgia, M., Parma, P., Mack, K.-H., de Ruiter, H. R., Fanti, R., Govoni, F., Tarchi, A., Giacintucci, S., and Markevitch, M. (2011). Dying radio galaxies in clusters. aap, 526:A148.

Muriel, H., Nicotra, M. A., and Lambas, D. G. (1995). The Luminosity Function of Elliptical Galaxies. aj, 110:1032.

Norris, R. P., Hopkins, A. M., Afonso, J., Brown, S., Condon, J. J., and others. (2011). EMU: Evolutionary Map of the Universe. pasa, 28:215-248.

O’Hara, T. B., Mohr, J. J., Bialek, J. J., and Evrard, A. E. (2006). Effects of Mergers and Core Structure on the Bulk Properties of Nearby Galaxy Clusters. apj, 639:64-80.

Oppermann, N., Junklewitz, H., Greiner, M., and others. (2015). Estimating extragalactic Faraday rotation. aap, 575:A118.

Oppermann, N., Junklewitz, H., Robbers, G., and others. (2012). An improved map of the Galactic Faraday sky. aap, 542:A93. 
O’Sullivan, S. P., Brown, S., Robishaw, T., Schnitzeler, D. H. F. M., and others. (2012). Complex Faraday depth structure of active galactic nuclei as revealed by broadband radio polarimetry. mnras, 421:3300-3315.

O’Sullivan, S. P. and Gabuzda, D. C. (2008). Parsec-Scale Investigation of the Magnetic Field Structure of Several AGN Jets. International Journal of Modern Physics D, 17:1553-1560.

Overzier, R., Lemson, G., Angulo, and others.. (2013). The Millennium Run Observatory: first light. mnras, 428:778-803.

Owen, F. N., Rudnick, L., Eilek, J., Rau, U., Bhatnagar, S., and Kogan, L. (2014). Wideband Very Large Array Observations of A2256. I. Continuum, Rotation Measure, and Spectral Imaging. apj, 794:24.

Owers, M. S., Baldry, I. K., Bauer, and others. (2013). Galaxy and Mass Assembly (GAMA): Witnessing the Assembly of the Cluster ABELL 1882. apj, 772:104.

Owers, M. S., Couch, W. J., and Nulsen, P. E. J. (2009). Substructure in the Cold Front Cluster Abell 3667. apj, 693:901-913.

Owers, M. S., Nulsen, P. E. J., and others. (2014). A Merger Shock in A2034. apj, 780:163.

Owers, M. S., Randall, S. W., Nulsen, P. E. J., Couch, W. J., David, L. P., and Kempner, J. C. (2011). The Dissection of Abell 2744: A Rich Cluster Growing Through Major and Minor Mergers. apj, 728:27.

Peebles, P. J. E. (1980). The large-scale structure of the universe.

Petrosian, V., Bykov, A., and Rephaeli, Y. (2008). Nonthermal Radiation Mechanisms. ssr, 134:191-206.

Pfrommer, C., Enßlin, T. A., and Springel, V. (2008). Simulating cosmic rays in clusters of galaxies - II. A unified scheme for radio halos and relics with predictions of the $\gamma$-ray emission. mnras, 385:1211-1241.

Pfrommer, C. and Jones, T. W. (2011). Radio Galaxy NGC 1265 Unveils the Accretion Shock Onto the Perseus Galaxy Cluster. apj, 730:22.

Pimbblet, K. A., Smail, I., Edge, A. C., O’Hely, E., Couch, W. J., and Zabludoff, A. I. (2006). The Las Campanas/Anglo-Australian Telescope Rich Cluster Survey - III. Spectroscopic studies of X-ray bright galaxy clusters at $\mathrm{z}^{\sim}$ 0.1. mnras, 366:645666. 
Pinkney, J., Roettiger, K., Burns, J. O., and Bird, C. M. (1996). Evaluation of Statistical Tests for Substructure in Clusters of Galaxies. apjs, 104:1.

Pinzke, A., Oh, S. P., and Pfrommer, C. (2015). Turbulence and Particle Acceleration in Giant Radio Halos: the Origin of Seed Electrons. ArXiv e-prints.

Pizzo, R. F. and de Bruyn, A. G. (2008). Diffuse Extended Emission and Galaxy Population of A2255. In Rector, T. A. and De Young, D. S., editors, Extragalactic Jets: Theory and Observation from Radio to Gamma Ray, volume 386 of Astronomical Society of the Pacific Conference Series, page 368.

Pizzo, R. F., de Bruyn, A. G., and Brentjens, M. A. (2009). Polarization In and Around the Galaxy Cluster Abell 2255. In Saikia, D. J., Green, D. A., Gupta, Y., and Venturi, T., editors, The Low-Frequency Radio Universe, volume 407 of Astronomical Society of the Pacific Conference Series, page 241.

Plagge, T., Benson, and others. (2010). Sunyaev-Zel'dovich Cluster Profiles Measured with the South Pole Telescope. apj, 716:1118-1135.

Planck Collaboration, Ade, P. A. R., Aghanim, N., Armitage-Caplan, C., Arnaud, M., Ashdown, M., Atrio-Barandela, F., Aumont, J., Baccigalupi, C., Banday, A. J., and et al. (2013). Planck 2013 results. XVI. Cosmological parameters. ArXiv e-prints.

Planck Collaboration, Ade, P. A. R., Aghanim, N., Arnaud, M., Ashdown, M., Aumont, J., Baccigalupi, C., Balbi, A., Banday, A. J., Barreiro, R. B., and et al. (2011). Planck early results. VIII. The all-sky early Sunyaev-Zeldovich cluster sample. aap, 536:A8.

Planck Collaboration, Ade, P. A. R., Aghanim, N., Arnaud, M., Ashdown, M., Aumont, J., Baccigalupi, C., Banday, A. J., Barreiro, R. B., Barrena, R., and et al. (2015). Planck 2015 results. XXVII. The Second Planck Catalogue of Sunyaev-Zeldovich Sources. ArXiv e-prints.

Poggianti, B. M., Fasano, G., Bettoni, and others. (2009). The Evolution of Spiral, S0, and Elliptical Galaxies in Clusters. apjl, 697:L137-L140.

Pratley, L. (2012). Astrophysics Honors Report. Master's thesis, Victoria University of Wellington.

Pratley, L., Johnston-Hollitt, M., Dehghan, S., and Sun, M. (2013). Using head-tail galaxies to constrain the intracluster magnetic field: an in-depth study of PKS J0334-3900. mnras, 432:243-257. 
Pratt, G. W., Croston, J. H., Arnaud, M., and Böhringer, H. (2009). Galaxy cluster Xray luminosity scaling relations from a representative local sample (REXCESS). aap, 498:361-378.

Prokhorov, D. A. and Churazov, E. M. (2014). Counting gamma rays in the directions of galaxy clusters. aap, 567:A93.

Protheroe, R. J. (1999). Acceleration and interaction of ultra high energy cosmic rays. In Duvernois, M. A., editor, Topics in Cosmic-Ray Astrophysics, page 247.

Pudritz, R. E., Hardcastle, M. J., and Gabuzda, D. C. (2012). Magnetic Fields in Astrophysical Jets: From Launch to Termination. ssr, 169:27-72.

Rau, U. and Cornwell, T. J. (2011). A multi-scale multi-frequency deconvolution algorithm for synthesis imaging in radio interferometry. aap, 532:A71.

Regos, E. and Geller, M. J. (1989). Infall patterns around rich clusters of galaxies. aj, 98:755-765.

Reid, A. D., Hunstead, R. W., Lemonon, L., and Pierre, M. M. (1999). Radio study of $\mathrm{X}$-ray clusters of galaxies - II. A1300: a post-merger cluster at $\mathrm{z}=0.31$ containing a halo and a relic. mnras, 302:571-581.

Reiprich, T. H., Basu, K., Ettori, S., Israel, H., Lovisari, L., Molendi, S., Pointecouteau, E., and Roncarelli, M. (2013). Outskirts of Galaxy Clusters. ssr, 177:195-245.

Rephaeli, Y. and Gruber, D. E. (1988). HEAO 1 hard X-ray observation of clusters of galaxies and intracluster magnetic fields. apj, 333:133-135.

Rines, K. and Diaferio, A. (2006). CIRS: Cluster Infall Regions in the Sloan Digital Sky Survey. I. Infall Patterns and Mass Profiles. aj, 132:1275-1297.

Rines, K., Geller, M. J., Diaferio, A., and Kurtz, M. J. (2013). Measuring the Ultimate Halo Mass of Galaxy Clusters: Redshifts and Mass Profiles from the Hectospec Cluster Survey (HeCS). apj, 767:15.

Rudnick, L. and Owen, F. N. (1976). Head-tail radio sources in clusters of galaxies. apjl, 203:L107-L111.

Russell, H. R., van Weeren, R. J., Edge, A. C., McNamara, B. R., Sanders, J. S., Fabian, A. C., Baum, S. A., Canning, R. E. A., Donahue, M., and O'Dea, C. P. (2011). A merger mystery: no extended radio emission in the merging cluster Abell 2146. mnras, 417:L1-L5. 
Sarazin, C. L. (1999). The Energy Spectrum of Primary Cosmic-Ray Electrons in Clusters of Galaxies and Inverse Compton Emission. apj, 520:529-547.

Sault, R. J., Teuben, P. J., and Wright, M. C. H. (1995). A Retrospective View of MIRIAD. In Shaw, R. A., Payne, H. E., and Hayes, J. J. E., editors, Astronomical Data Analysis Software and Systems IV, volume 77 of Astronomical Society of the Pacific Conference Series, page 433.

Schlickeiser, R., Sievers, A., and Thiemann, H. (1987). The diffuse radio emission from the Coma cluster. aap, 182:21-35.

Schwope, A., Hasinger, G., Lehmann, I., Schwarz, R., Brunner, H., Neizvestny, S., Ugryumov, A., Balega, Y., Trümper, J., and Voges, W. (2000). The ROSAT Bright Survey: II. Catalogue of all high-galactic latitude RASS sources with PSPC countrate CR $>0.2 \mathrm{~s}^{-1}$. Astronomische Nachrichten, 321:1-52.

Serra, A. L. (2014). Causticapp. http://personalpages.to.infn.it/ serra/ causticapp.html. Accessed: Jan-2014.

Serra, A. L. and Diaferio, A. (2013). Identification of Members in the Central and Outer Regions of Galaxy Clusters. apj, 768:116.

Serra, A. L., Diaferio, A., Murante, G., and Borgani, S. (2011). Measuring the escape velocity and mass profiles of galaxy clusters beyond their virial radius. mnras, 412:800-816.

Shakouri, S., Johnston-Hollitt, M., and Pratt, G. W. (2016). The ATCA REXCESS Diffuse Emission Survey (ARDES) - I. Detection of a giant radio halo and a likely radio relic. mnras, 459:2525-2538.

Shimwell, T. W., Brown, S., Feain, I. J., Feretti, L., Gaensler, B. M., and Lage, C. (2014). Deep radio observations of the radio halo of the bullet cluster 1E 065755.8. mnras, 440:2901-2915.

Sijbring, L. G. (1993). A radio continuum and HI line study of the perseus cluster. $\mathrm{PhD}$ thesis, Groningen: Rijksuniversiteit.

Slee, O. B., Roy, A. L., Murgia, M., Andernach, H., and Ehle, M. (2001). Four Extreme Relic Radio Sources in Clusters of Galaxies. aj, 122:1172-1193.

Smolčić, V., Schinnerer, E., Finoguenov, A., Sakelliou, I., and others. (2007). A WideAngle Tail Radio Galaxy in the COSMOS Field: Evidence for Cluster Formation. apjs, 172:295-313. 
Snellen, I. A. G., Bremer, M. N., Schilizzi, R. T., Miley, G. K., and van Ojik, R. (1996). The R-band Hubble diagram for gigahertz peaked spectrum radio galaxies. mnras, 279:1294-1302.

Sommer, M. W. and Basu, K. (2014). A comparative study of radio halo occurrence in SZ and X-ray selected galaxy cluster samples. mnras, 437:2163-2179.

Strauss, M. A., Huchra, J. P., Davis, M., Yahil, A., Fisher, K. B., and Tonry, J. (1992). A redshift survey of IRAS galaxies. VII - The infrared and redshift data for the 1.936 Jansky sample. apjs, 83:29-63.

Sunyaev, R. A. and Zeldovich, Y. B. (1970). The Spectrum of Primordial Radiation, its Distortions and their Significance. Comments on Astrophysics and Space Physics, 2:66.

Sunyaev, R. A. and Zeldovich, Y. B. (1972). The Observations of Relic Radiation as a Test of the Nature of X-Ray Radiation from the Clusters of Galaxies. Comments on Astrophysics and Space Physics, 4:173.

Teague, P. F., Carter, D., and Gray, P. M. (1990). The dynamics and structure of rich clusters of galaxies. I - Velocity data. apjs, 72:715-753.

Thierbach, M., Klein, U., and Wielebinski, R. (2003). The diffuse radio emission from the Coma cluster at $2.675 \mathrm{GHz}$ and $4.85 \mathrm{GHz}$. aap, 397:53-61.

Tingay, S. J., Goeke, R., Bowman, J. D., Emrich, D., and others. (2013). The Murchison Widefield Array: The Square Kilometre Array Precursor at Low Radio Frequencies. pasa, 30:7.

Tonry, J. and Davis, M. (1979). A survey of galaxy redshifts. I - Data reduction techniques. aj, 84:1511-1525.

Tramacere, A. and Vecchio, C. (2013). $\gamma$-ray DBSCAN: a clustering algorithm applied to Fermi-LAT $\gamma$-ray data. I. Detection performances with real and simulated data. aap, 549:A138.

Tribble, P. C. (1991). Radio emission in a random magnetic field - Radio halos and the structure of the magnetic field in the Coma cluster. mnras, 253:147-152.

Vacca, V., Feretti, L., Giovannini, G., Govoni, F., Murgia, M., Perley, R. A., and Clarke, T. E. (2014). Spectral index image of the radio halo in the cluster Abell 520, which hosts the famous bow shock. aap, 561:A52. 
Vacca, V., Govoni, F., Murgia, M., Giovannini, G., Feretti, L., Tugnoli, M., Verheijen, M. A., and Taylor, G. B. (2011). Discovery of diffuse radio emission in the galaxy cluster A1689. aap, 535:A82.

Vallee, J. P., MacLeod, M. J., and Broten, N. W. (1986). A large-scale magnetic feature in the galaxy cluster A 2319. aap, 156:386-390.

van Haarlem, M. and van de Weygaert, R. (1993). Velocity Fields and Alignments of Clusters in Gravitational Instability Scenarios. apj, 418:544.

van Haarlem, M. P., Wise, M. W., Gunst, A. W., Heald, G., McKean, J. P., and others. (2013). LOFAR: The LOw-Frequency ARray. aap, 556:A2.

van Weeren, R. J., Brüggen, M., Röttgering, H. J. A., Hoeft, M., Nuza, S. E., and Intema, H. T. (2011a). Radio continuum observations of new radio halos and relics from the NVSS and WENSS surveys. Relic orientations, cluster X-ray luminosity, and redshift distributions. aap, 533:A35.

van Weeren, R. J., Hoeft, M., Röttgering, H. J. A., Brüggen, M., Intema, H. T., and van Velzen, S. (2011b). A double radio relic in the merging galaxy cluster $\mathrm{ZwCl}$ 0008.8+5215. aap, 528:A38.

van Weeren, R. J., Intema, H. T., Lal, D. V., and others. (2014a). A Distant Radio Mini-halo in the Phoenix Galaxy Cluster. apjl, 786:L17.

van Weeren, R. J., Intema, H. T., Lal, D. V., and others. (2014b). The Discovery of a Radio Halo in PLCK G147.3-16.6 at $\mathrm{z}=0.65$. apjl, 781:L32.

van Weeren, R. J., Intema, H. T., Oonk, J. B. R., Röttgering, H. J. A., and Clarke, T. E. (2009). The discovery of diffuse steep spectrum sources in Abell 2256. aap, 508:1269-1273.

van Weeren, R. J., Röttgering, H. J. A., Brüggen, M., and Hoeft, M. (2010). Particle Acceleration on Megaparsec Scales in a Merging Galaxy Cluster. Science, 330:347-.

Vazza, F., Brüggen, M., Gheller, C., and Wang, P. (2014). On the amplification of magnetic fields in cosmic filaments and galaxy clusters. mnras, 445:3706-3722.

Venturi, T., Bardelli, S., Dallacasa, D., Brunetti, G., Giacintucci, S., Hunstead, R. W., and Morganti, R. (2003). The radio halo in the merging cluster A3562. aap, 402:913-920.

Venturi, T., Giacintucci, S., Brunetti, and others. (2007). GMRT radio halo survey in galaxy clusters at $\mathrm{z}=0.2-0.4$. I. The REFLEX sub-sample. aap, 463:937-947. 
Venturi, T., Giacintucci, S., Dallacasa, D., Cassano, R., Brunetti, G., Bardelli, S., and Setti, G. (2008). GMRT radio halo survey in galaxy clusters at $z=0.2-0.4$. II. The eBCS clusters and analysis of the complete sample. aap, 484:327-340.

Vestrand, W. T. (1980). On the role of cosmic ray protons in two types of extragalactic objects. PhD thesis, Maryland Univ., College Park.

Wayth, R. B., Lenc, E., Bell, M. E., and others. (2015). GLEAM: The GaLactic and Extragalactic All-Sky MWA Survey. pasa, 32:25.

Weißmann, A., Böhringer, H., Šuhada, R., and Ameglio, S. (2013). Studying the properties of galaxy cluster morphology estimators. aap, 549:A19.

Whiting, M. T. (2012). DUCHAMP: a 3D source finder for spectral-line data. mnras, 421:3242-3256.

Willson, M. A. G. (1970). Radio observations of the cluster of galaxies in Coma Berenices - the 5C4 survey. mnras, 151:1-44.

Wilson, W. E., Ferris, R. H., Axtens, P., Brown, A., and others. (2011). The Australia Telescope Compact Array Broad-band Backend: description and first results. $m n$ ras, 416:832-856. 

$\S A$. Catalogue of the ARDES Radio Sources 
总

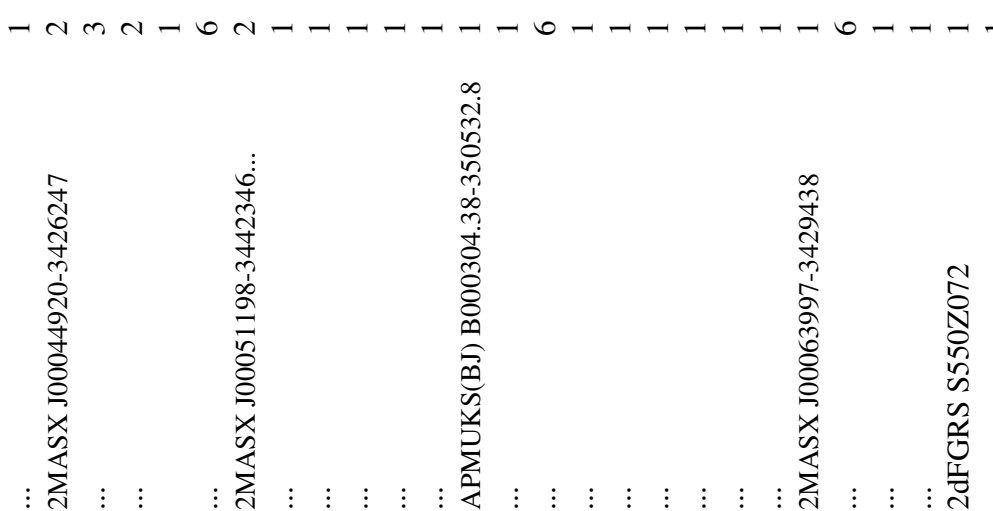

$: \overrightarrow{\widetilde{\sigma}}: \quad: \quad: \quad:$

$\underset{0}{\stackrel{7}{0}}:$

$: \frac{\mathfrak{f}}{2}$

$: \begin{array}{ll}\vec{m} \\ \infty \\ 0\end{array}:$

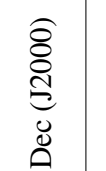

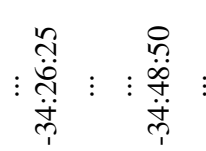

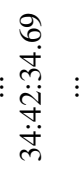

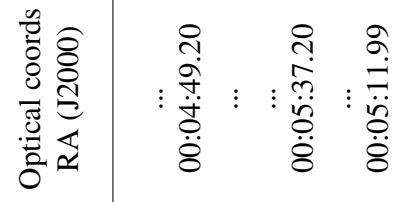

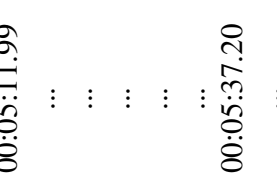

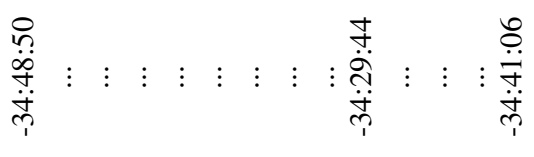

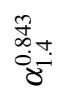

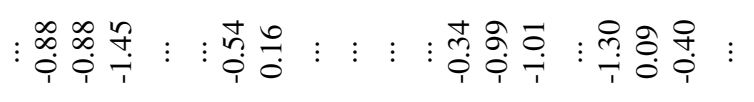

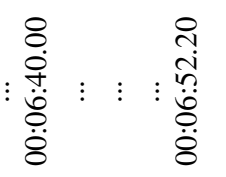

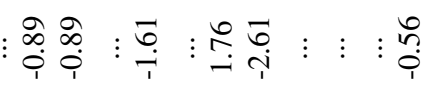

$\stackrel{\substack{0 \\ \infty}}{\stackrel{0}{0}}$

:

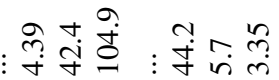

ํㅗㄴ

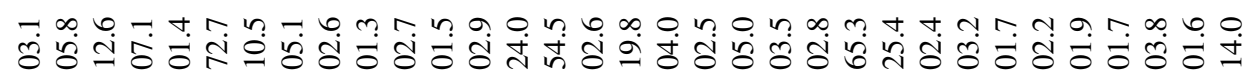

홍

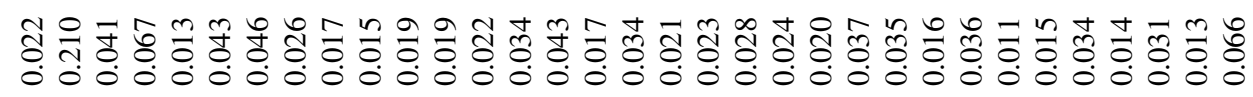

$\stackrel{+}{\stackrel{2}{\rightleftarrows}}$

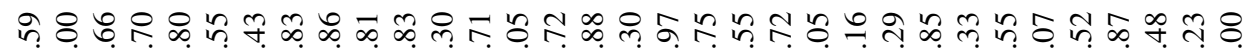

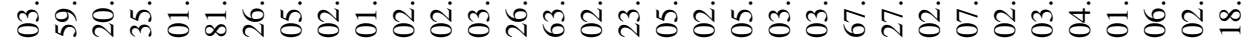

퐁

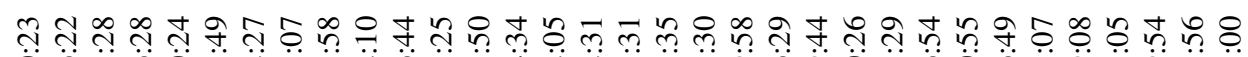

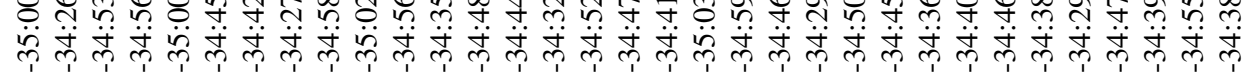

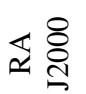

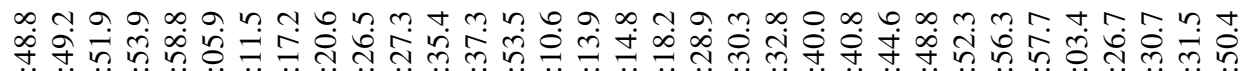

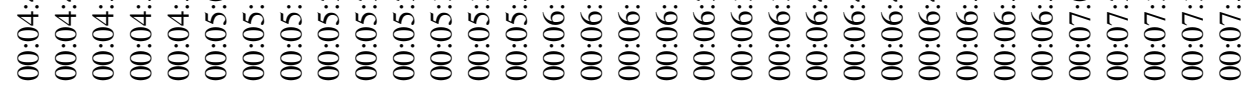

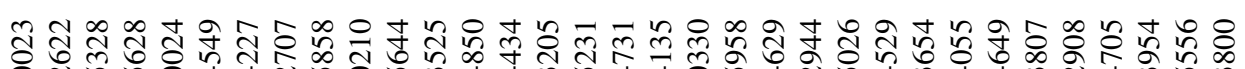

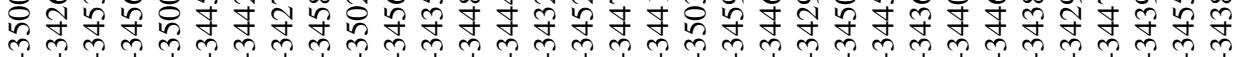




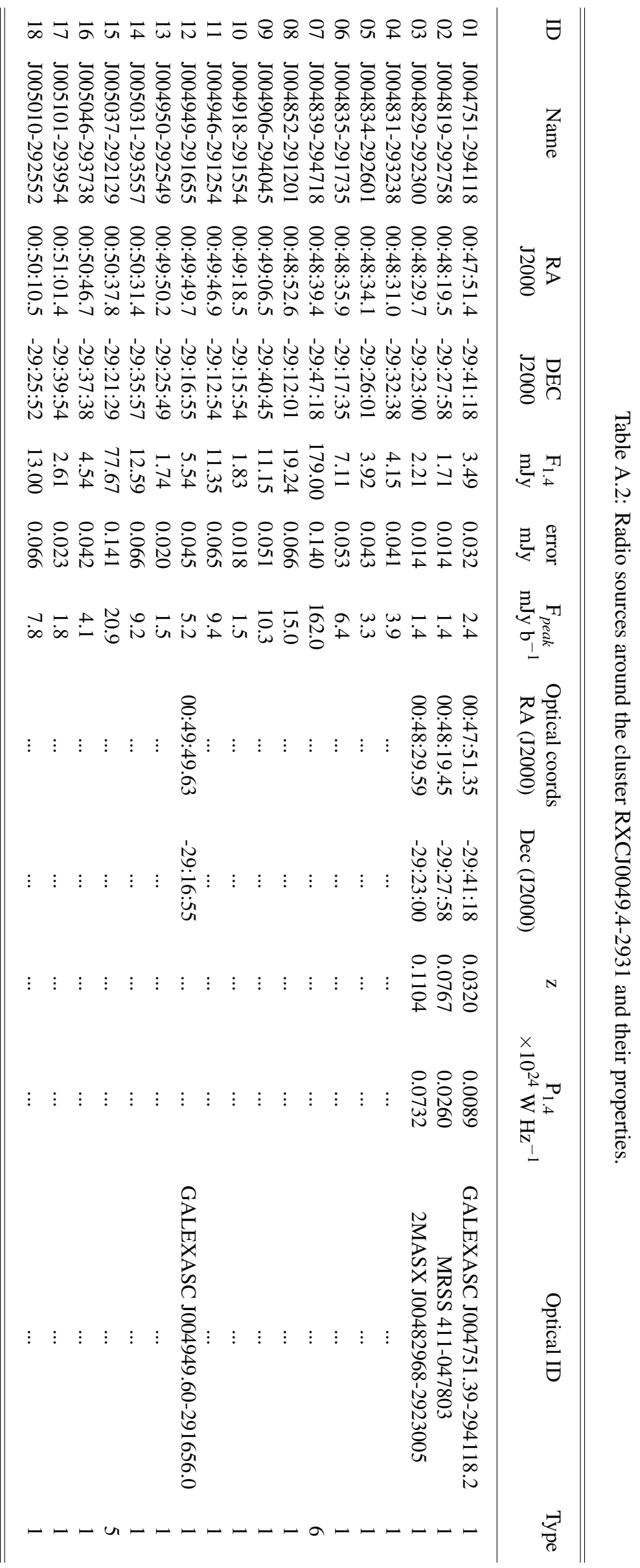




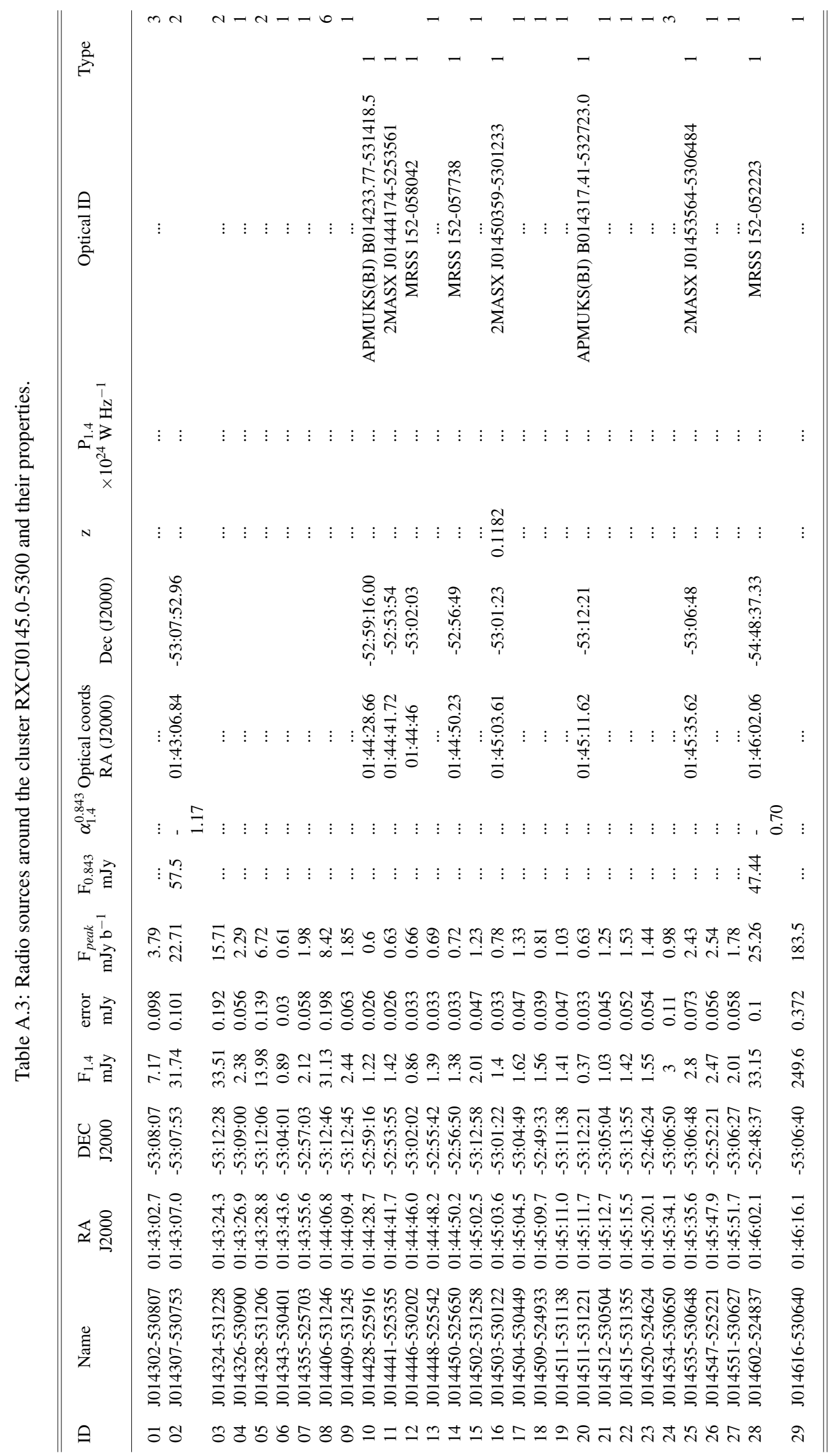




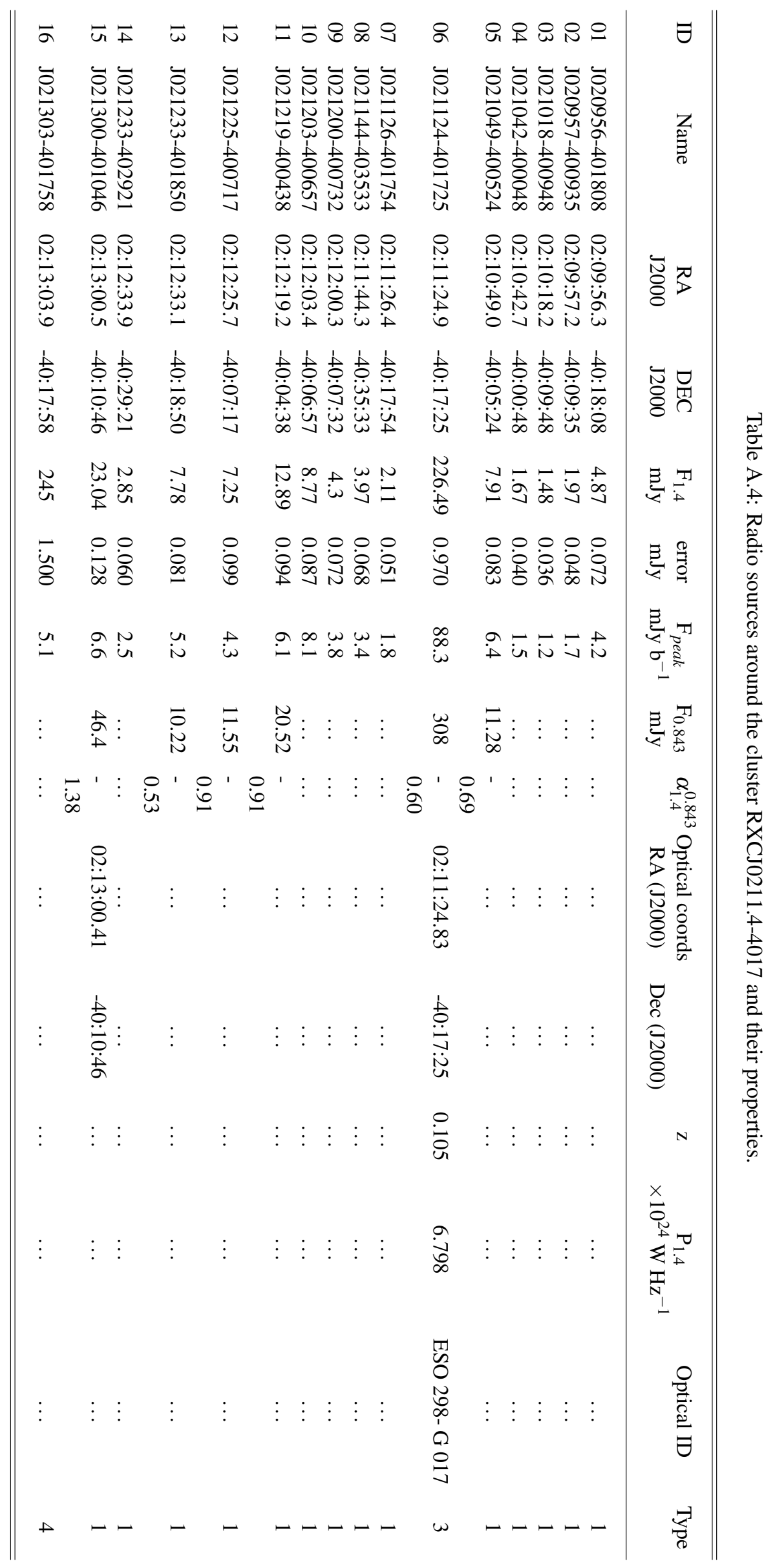




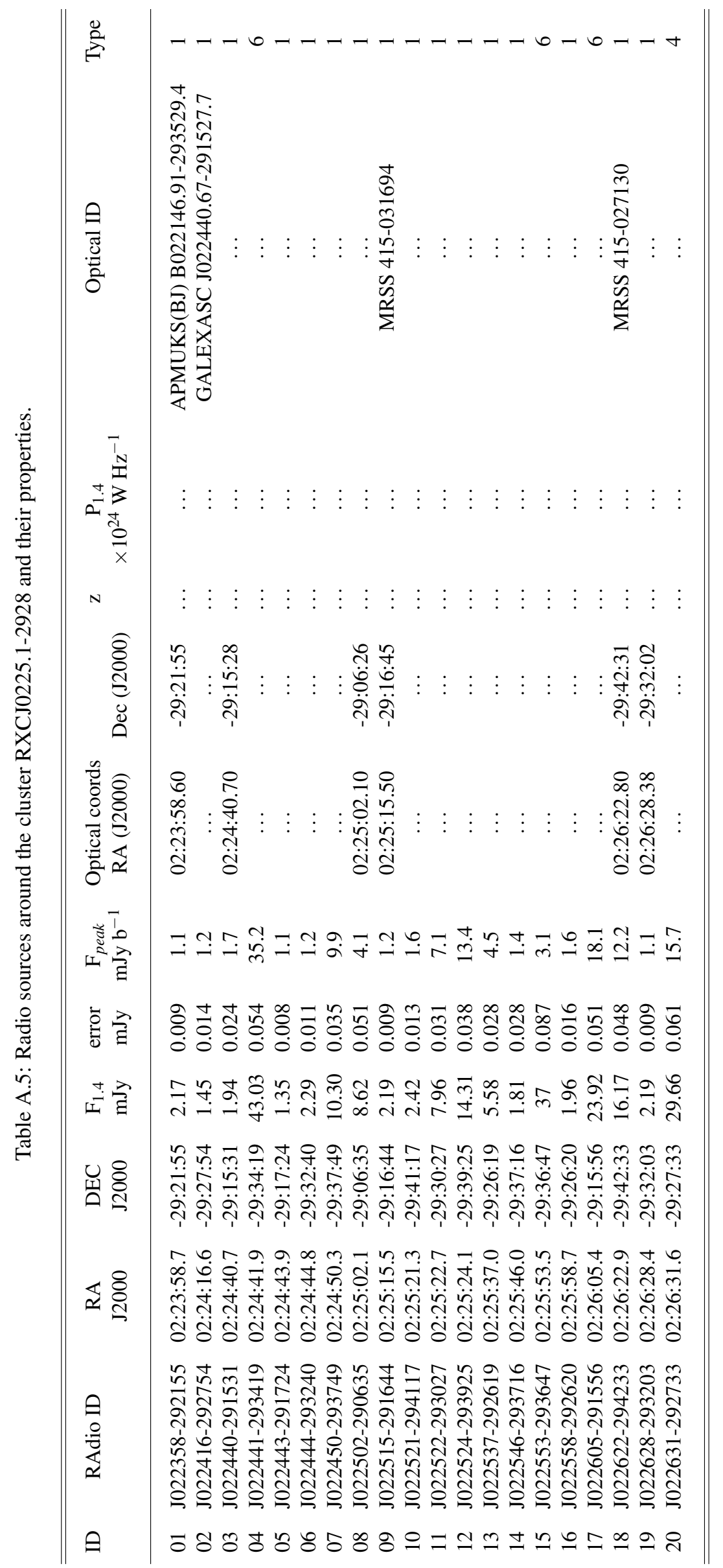




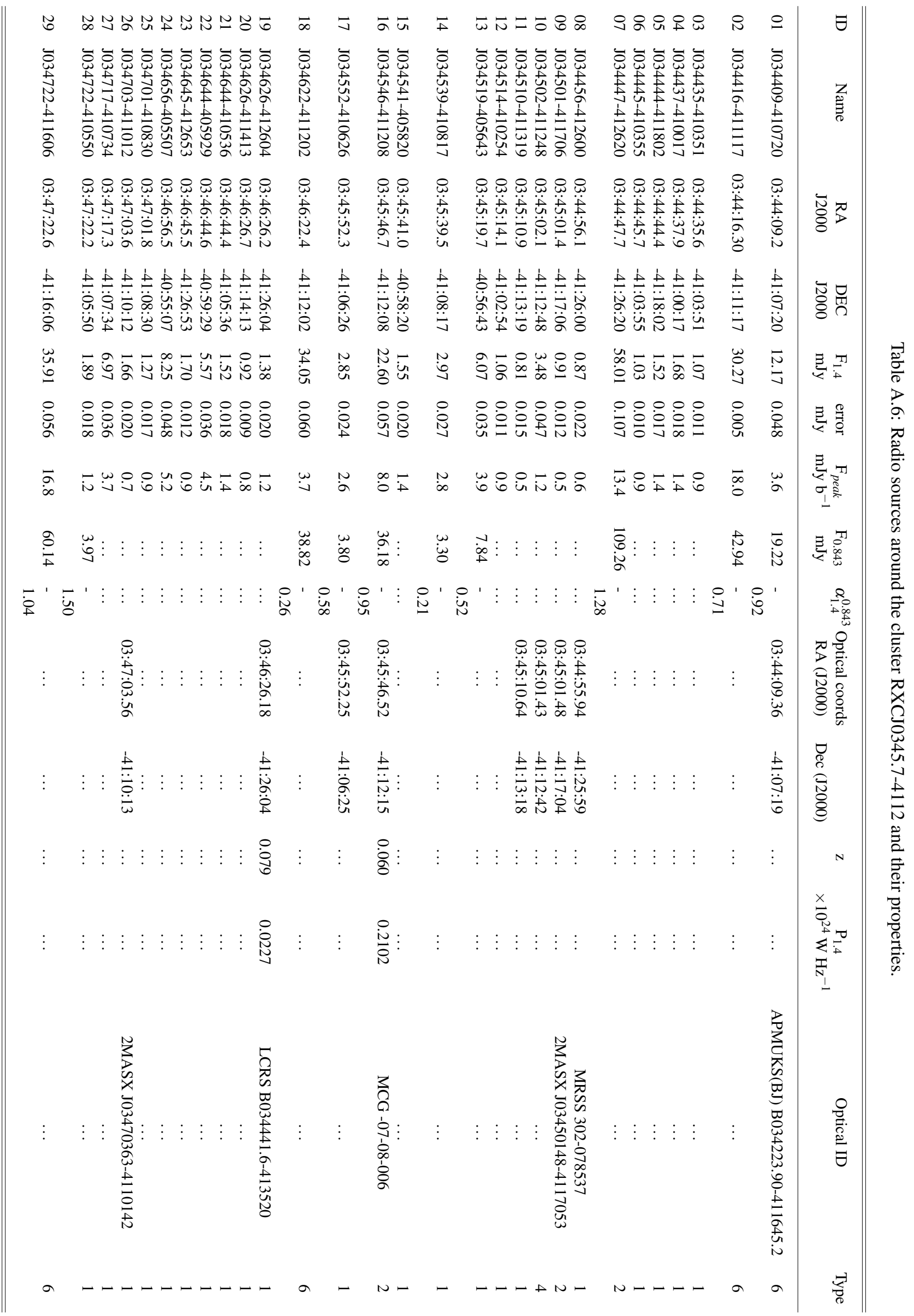




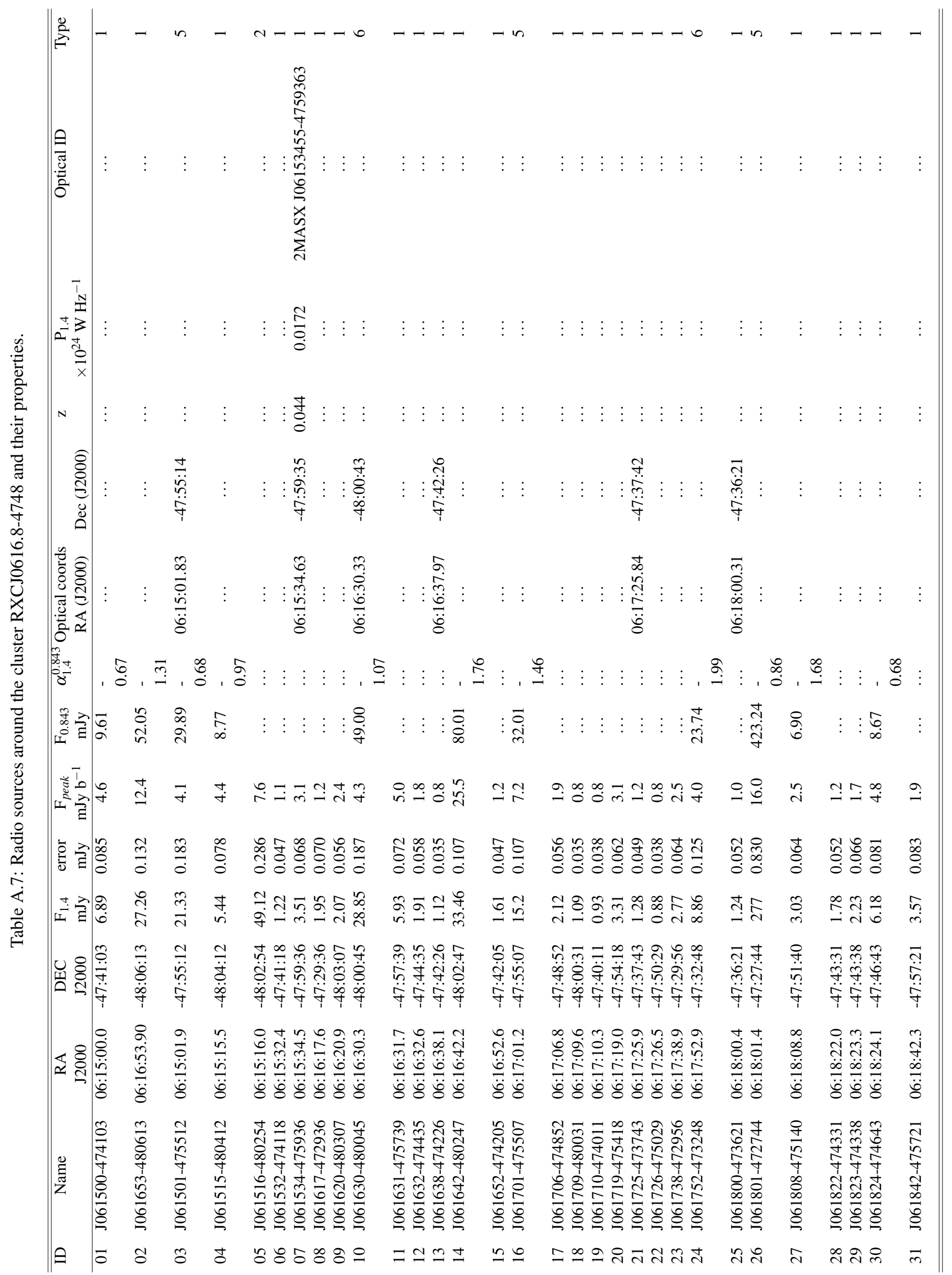




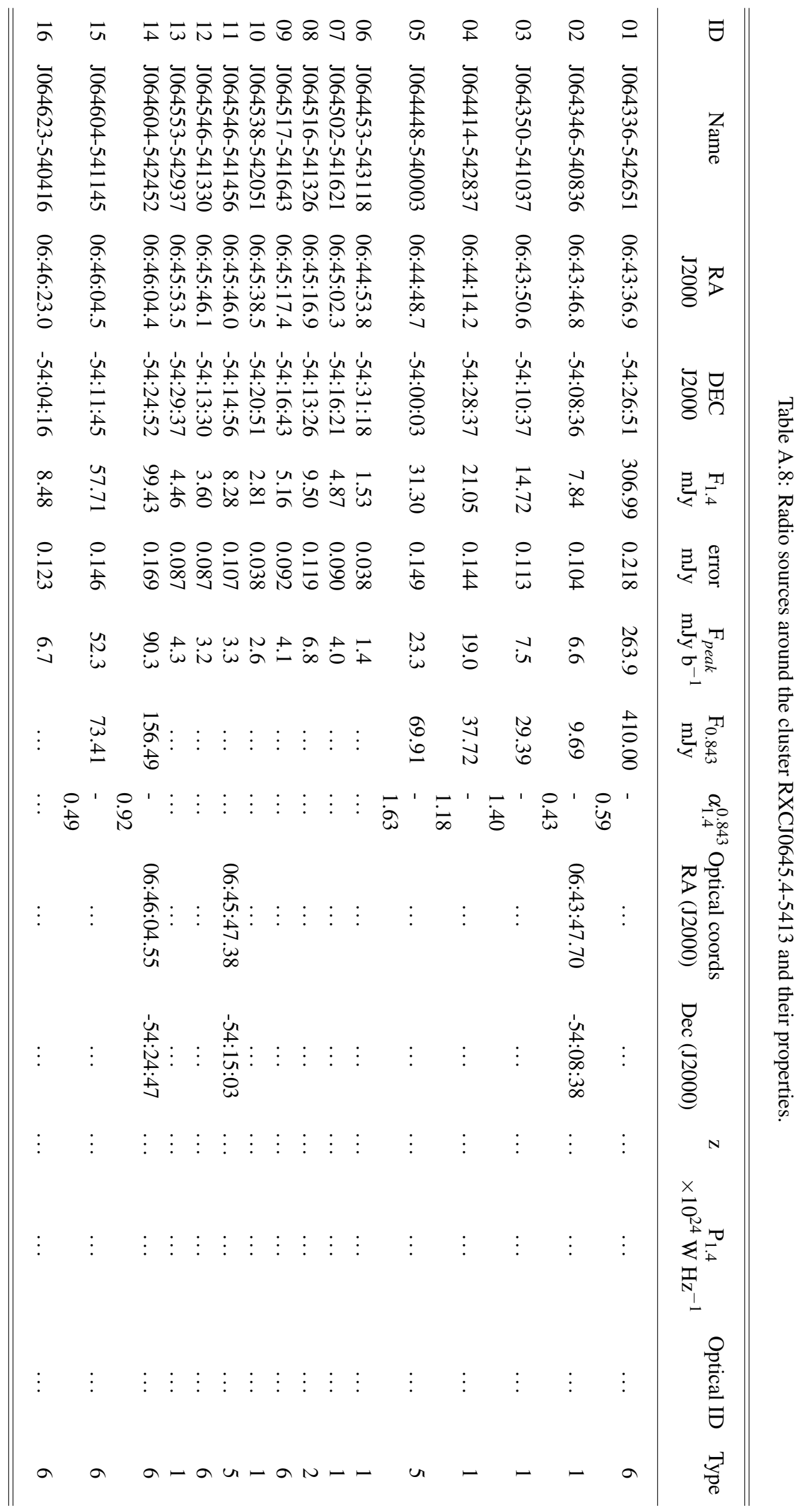




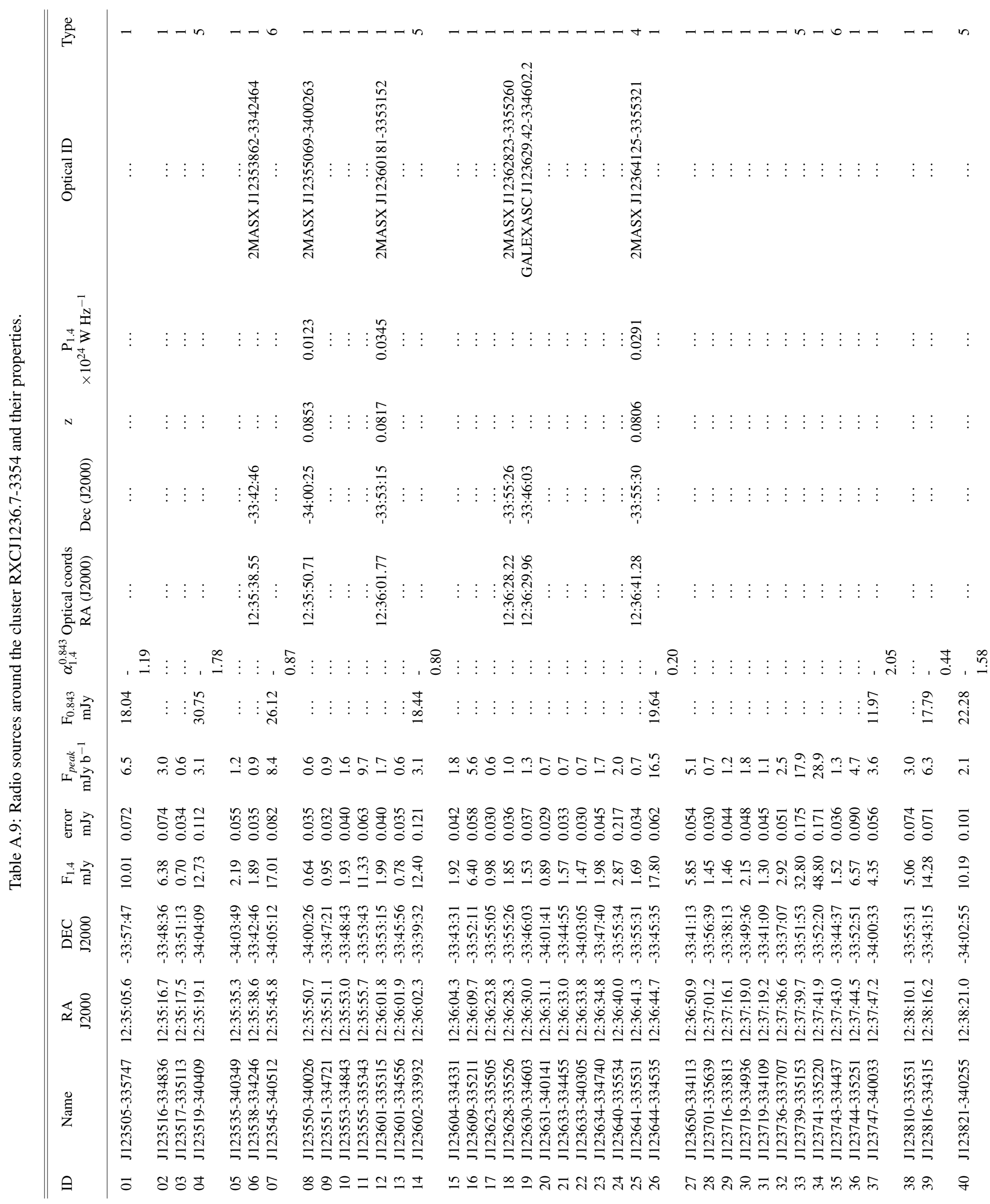




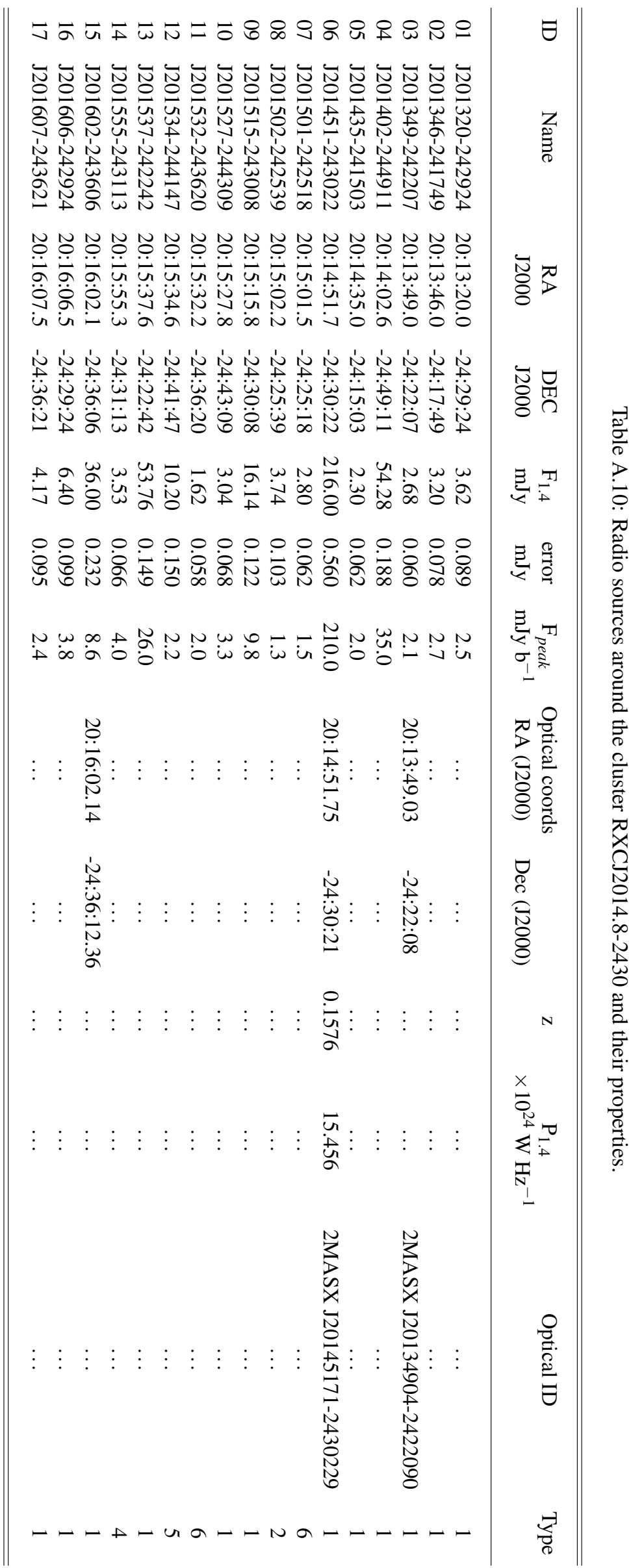




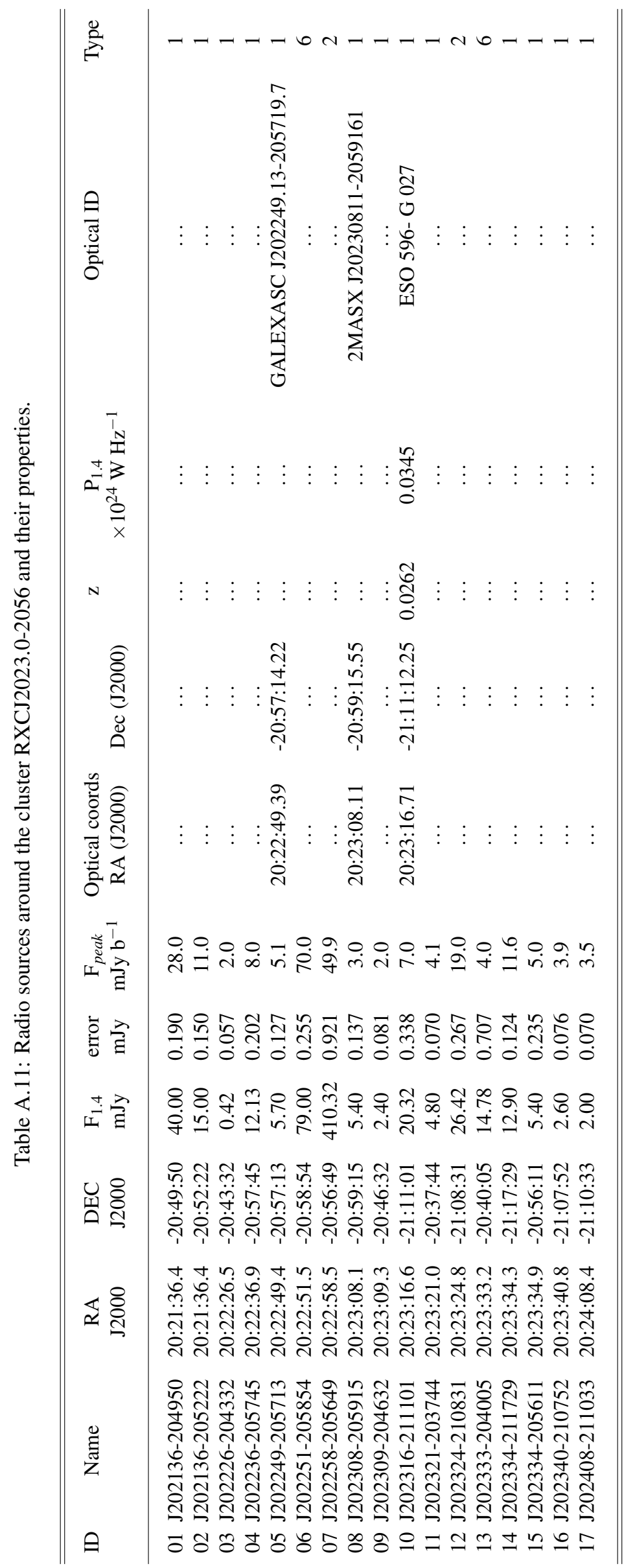




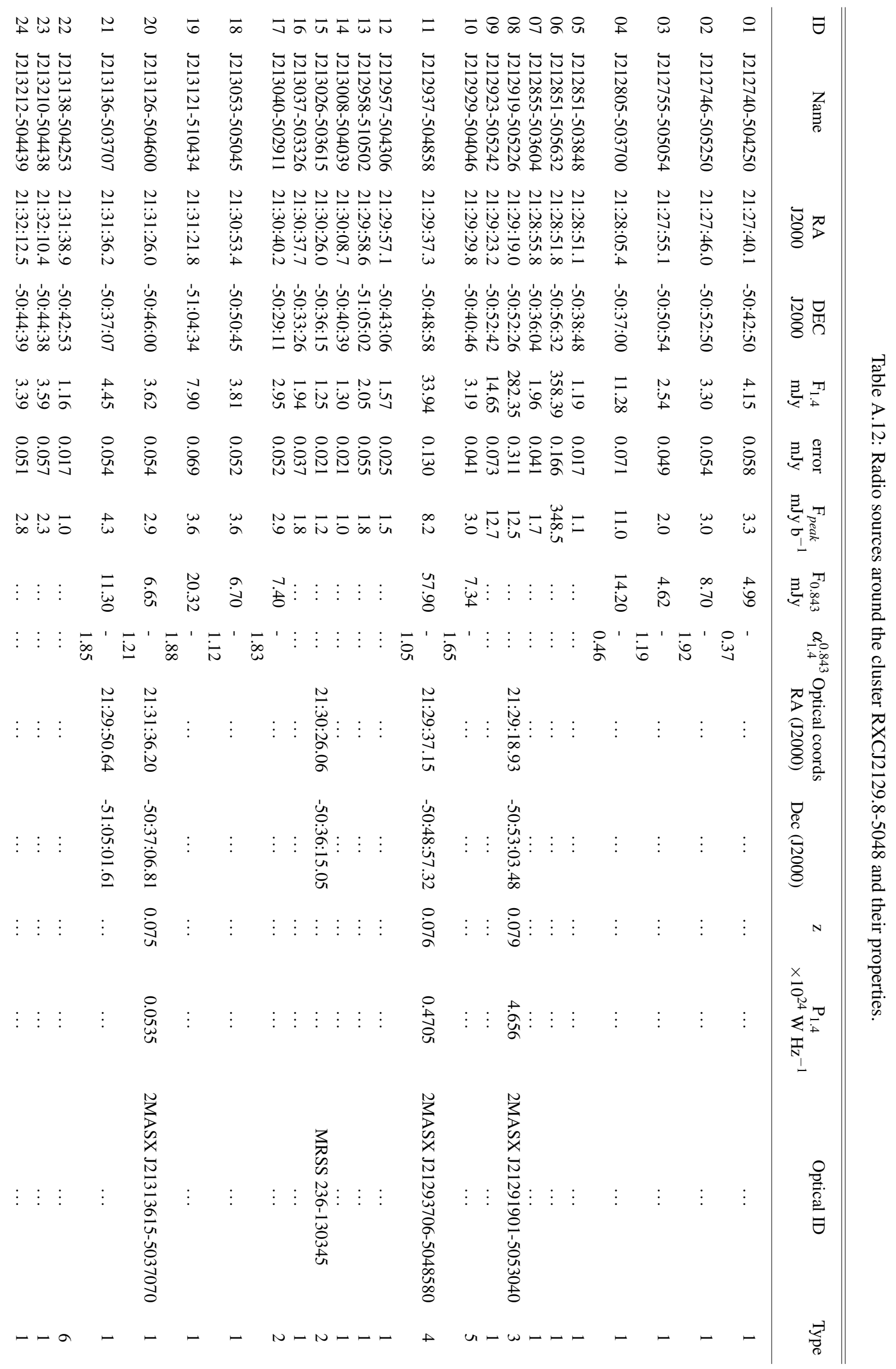




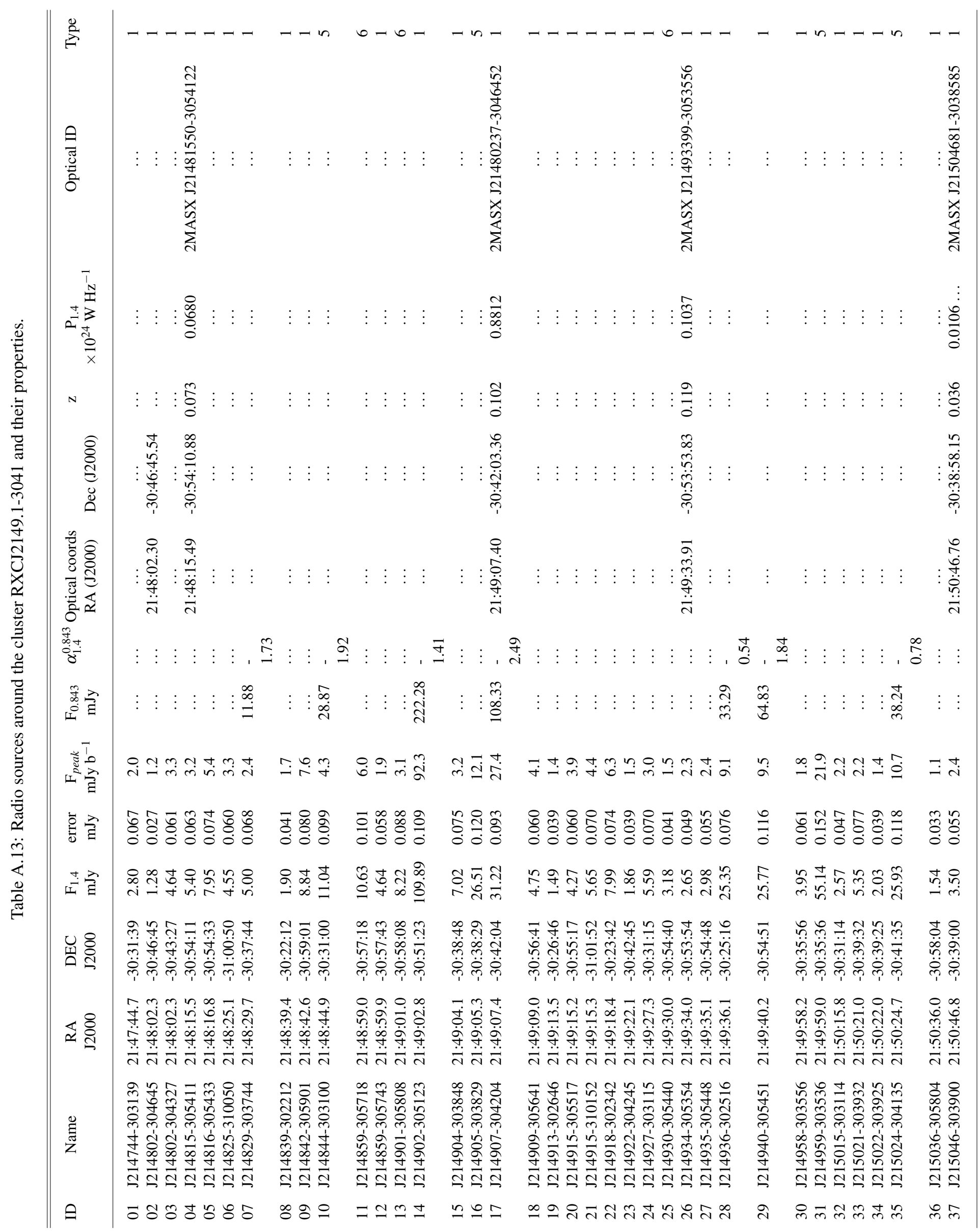




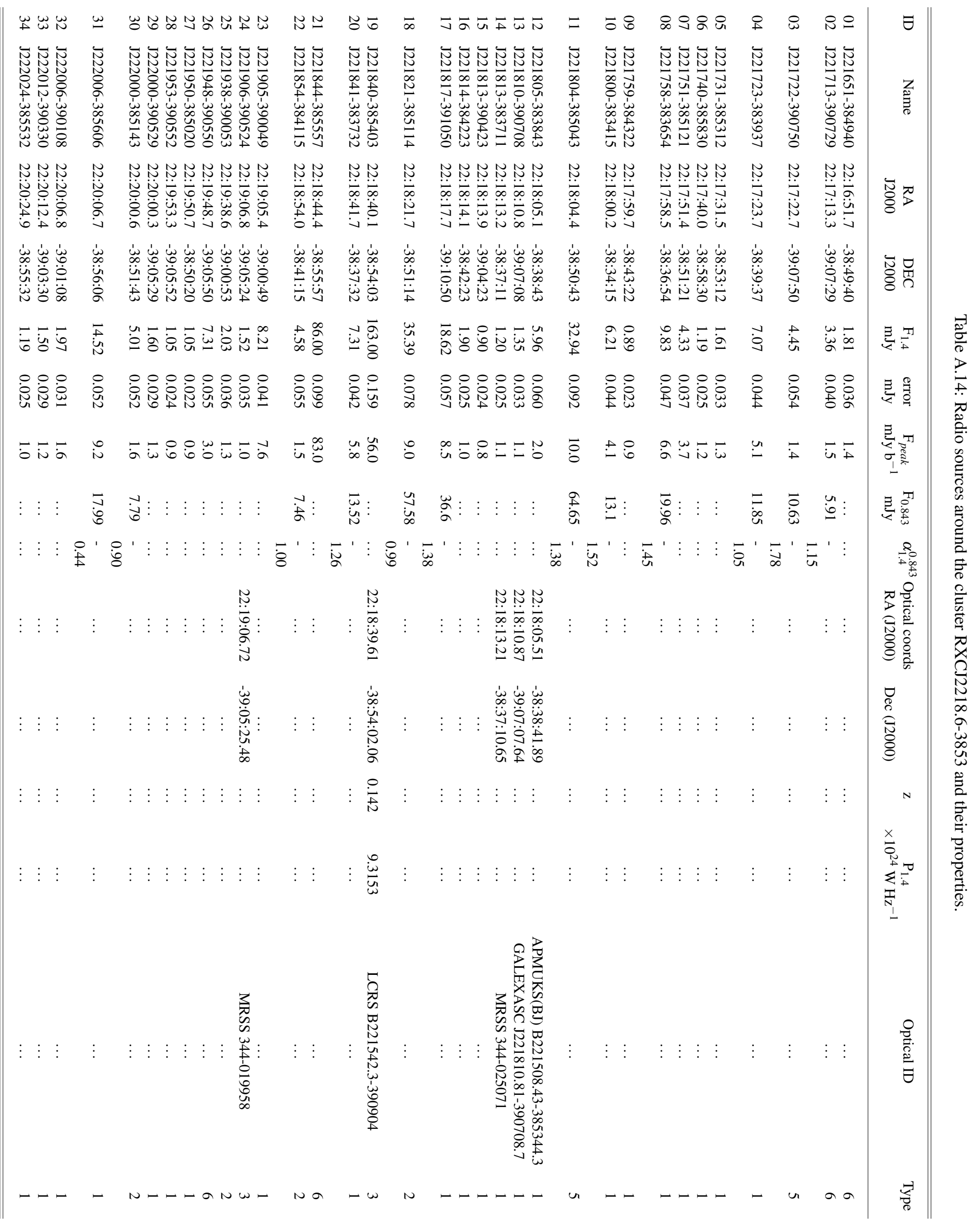




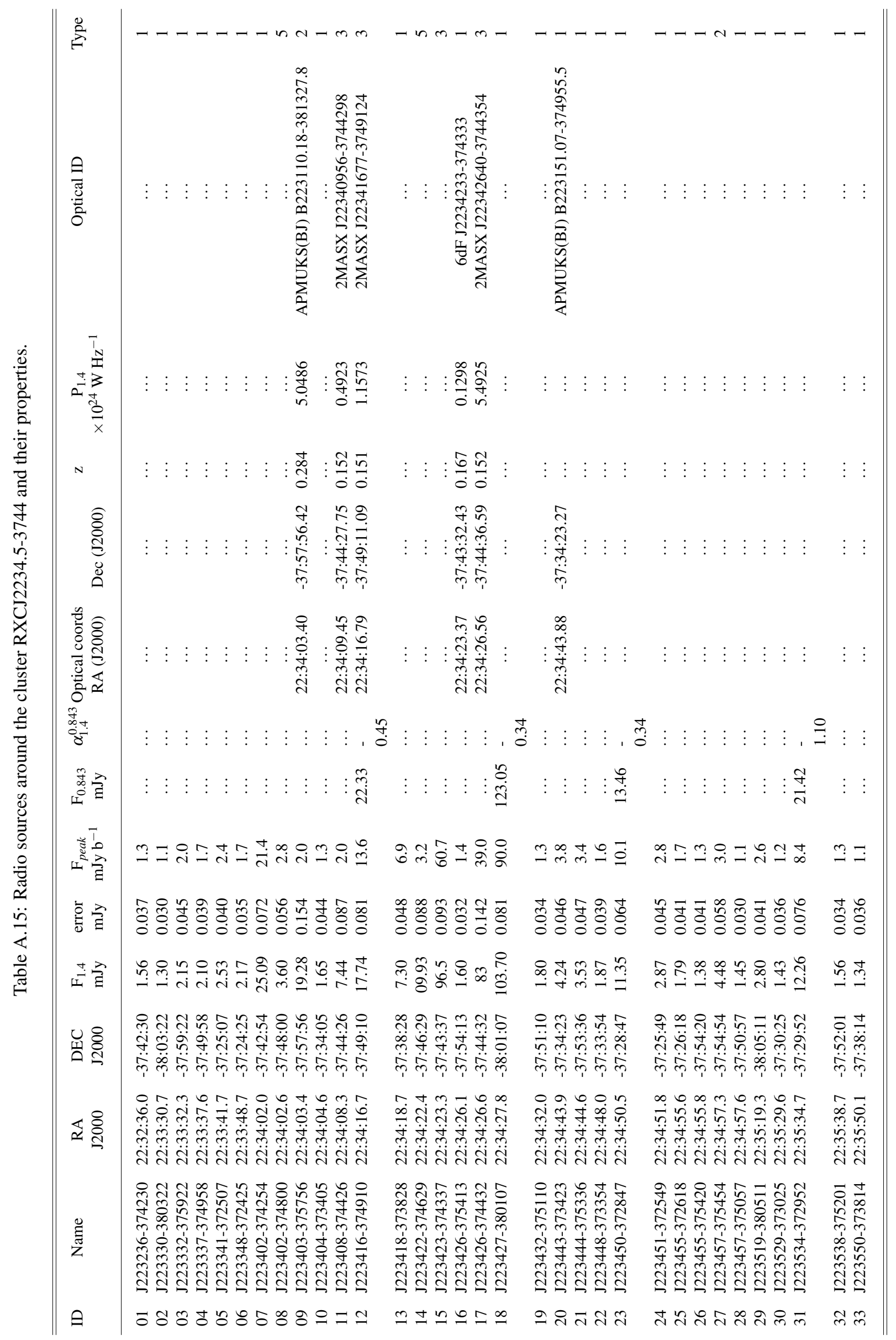




\section{$\S$ B. List of All Detetcted Radio Halos}




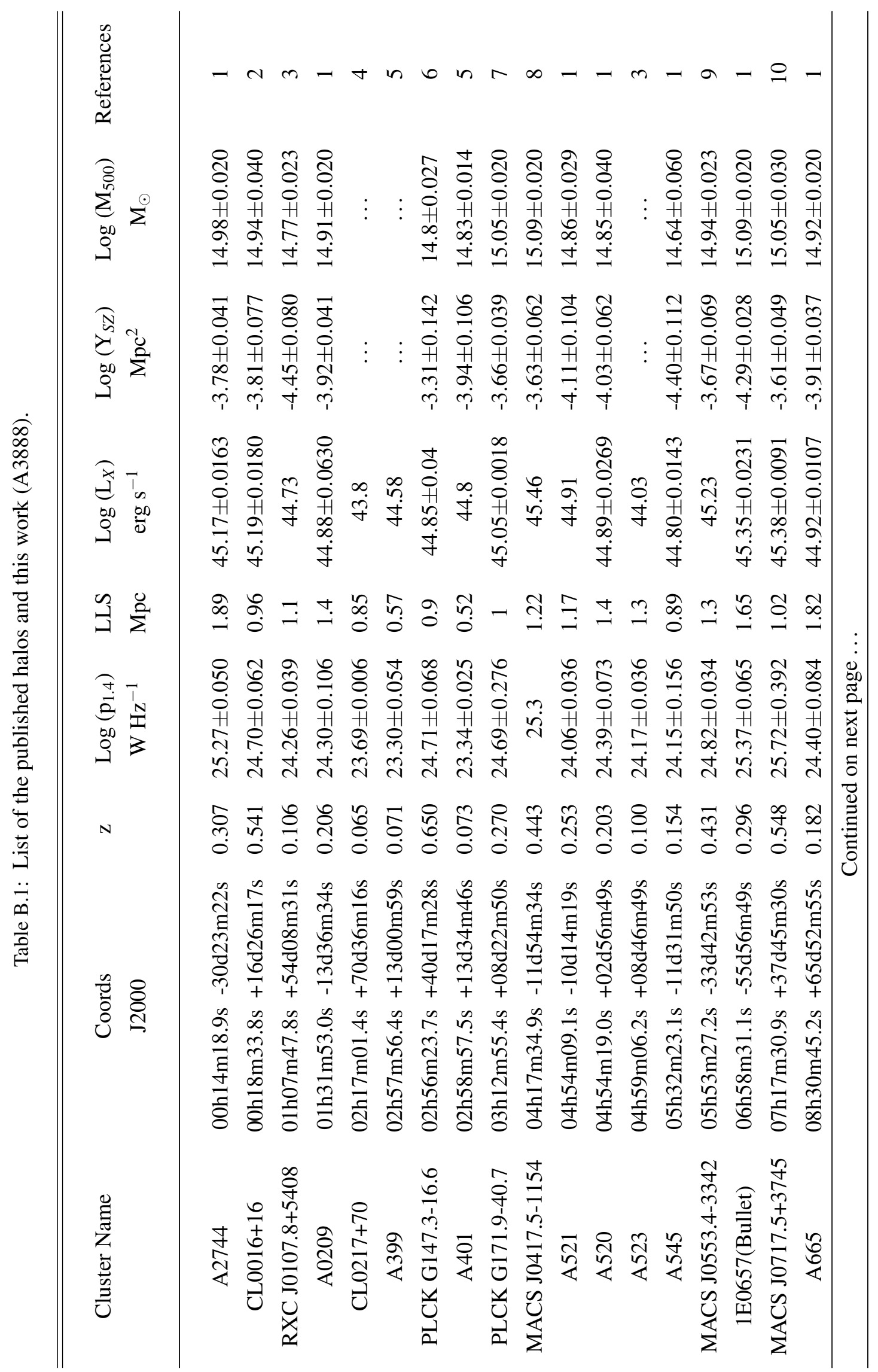




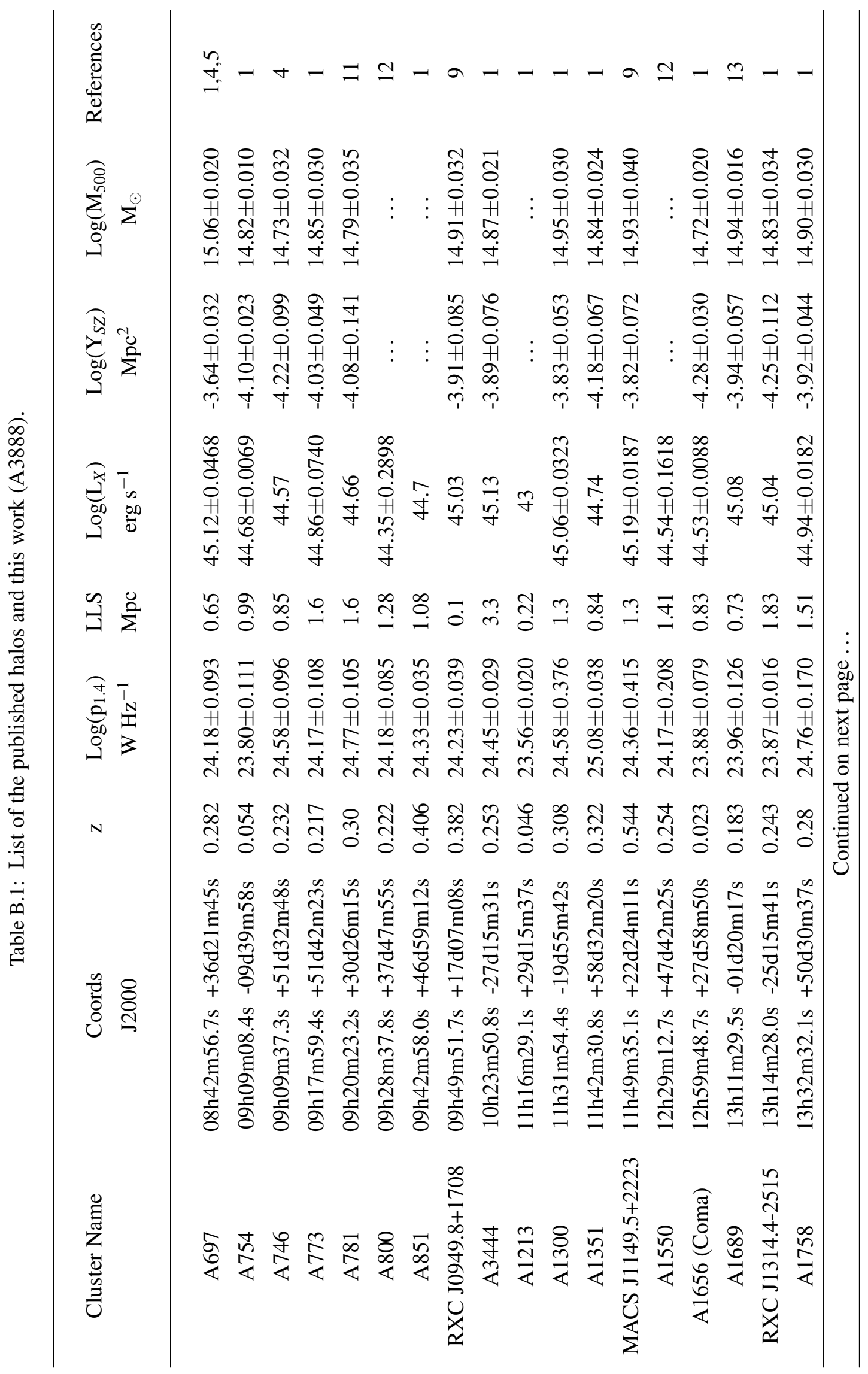




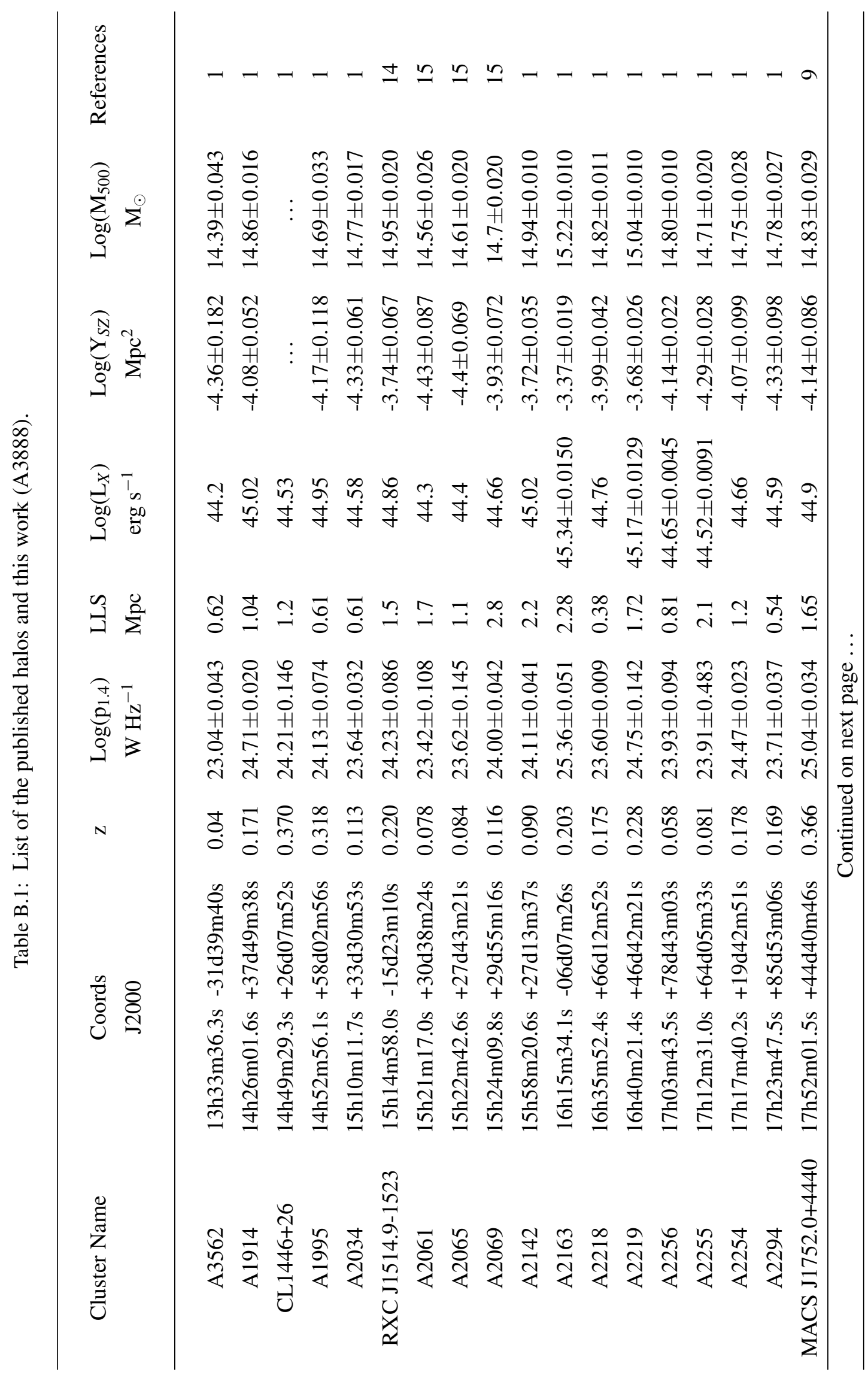




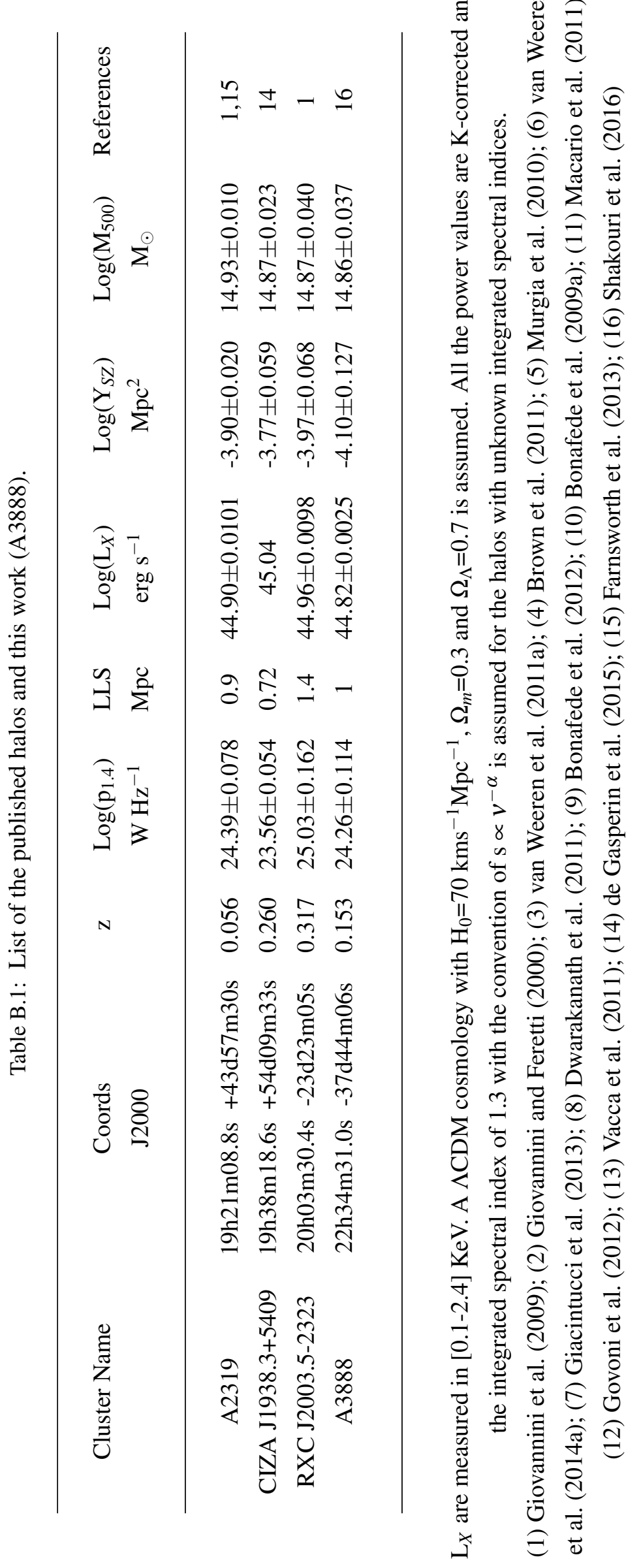





\section{$\S$ C. Morphology Estimators in X-ray Images}

The X-ray surface brightness distribution is widely used to infer the dynamical state of the galaxy clusters. The centroid shift, $w$, measures the variation of the centre of the mass (centroid) in different apertures. The offset between the centroid and the X-ray peak is measured in 10 aperture sizes in the range of $0.1-1 R_{500}$. The final $w$ is the standard deviation of all the measured separations in units of $R_{500}$ (Mohr et al., 1993; O'Hara et al., 2006). The centroid shift parameter is given by:

$$
w=\left[\frac{1}{N-1} \sum\left(\Delta_{i}-\langle\Delta\rangle\right)^{2}\right]^{1 / 2} \times \frac{1}{R_{500}}
$$

where $\Delta_{i}$ is the distance between the mean centroid and the centroid of the $i$ th aperture The other X-ray substructure estimator parameter is the third order power ratio. The power ratio method was introduced by Buote and Tsai (1995) with the aim to infer the dynamical state of the cluster from the X-ray surface brightness to define the projected mass distribution and substructure in the ICM.

Power ratio measurements are based on a 2D multipole decomposition of the clusters gravitational potential. The X-ray surface brightness is used to trace the mass density distribution to provide moments which are defined as power ratios when are normalised to zeroth moment. Higher order of power ratios describe finer structures. The powers are estimated within $\mathrm{R}_{500}$ with the aperture centred on the mass centroid. The third order power ratio $\mathrm{p}_{3} / \mathrm{p}_{0}$ is the lowest moment which provides a clear information about the substructure and it is more sensitive to the ICM asymmetries (Buote and Tsai, 1995). The $P_{m}$ moments are determined as follows:

$$
\begin{gathered}
P_{0}=\left[a_{0} \ln \left(R_{\mathrm{ap}}\right)\right]^{2} \\
P_{m}=\frac{1}{2 m^{2} R_{\mathrm{ap}}^{2 m}}\left(a_{m}^{2}+b_{m}^{2}\right)
\end{gathered}
$$

where $R_{\text {ap }}$ is the aperture radius. The moments $a_{m}$ and $b_{m}$ are calculated by:

$$
a_{m}(r)=\int_{r \leq R_{\mathrm{ap}}} d \vec{x} S(\vec{x}) r^{m} \cos (m \phi)
$$

and

$$
b_{m}(r)=\int_{r \leq R_{\mathrm{ap}}} d \vec{x} S(\vec{x}) r^{m} \sin (m \phi),
$$

where $S(\vec{x})$ is the X-ray surface brightness, and the integral extends over all pixels 
inside the aperture radius and $a_{0}$ is the total intensity inside the aperture radius. 


\section{$\S$ D. AAOmega Redshift Measurements}




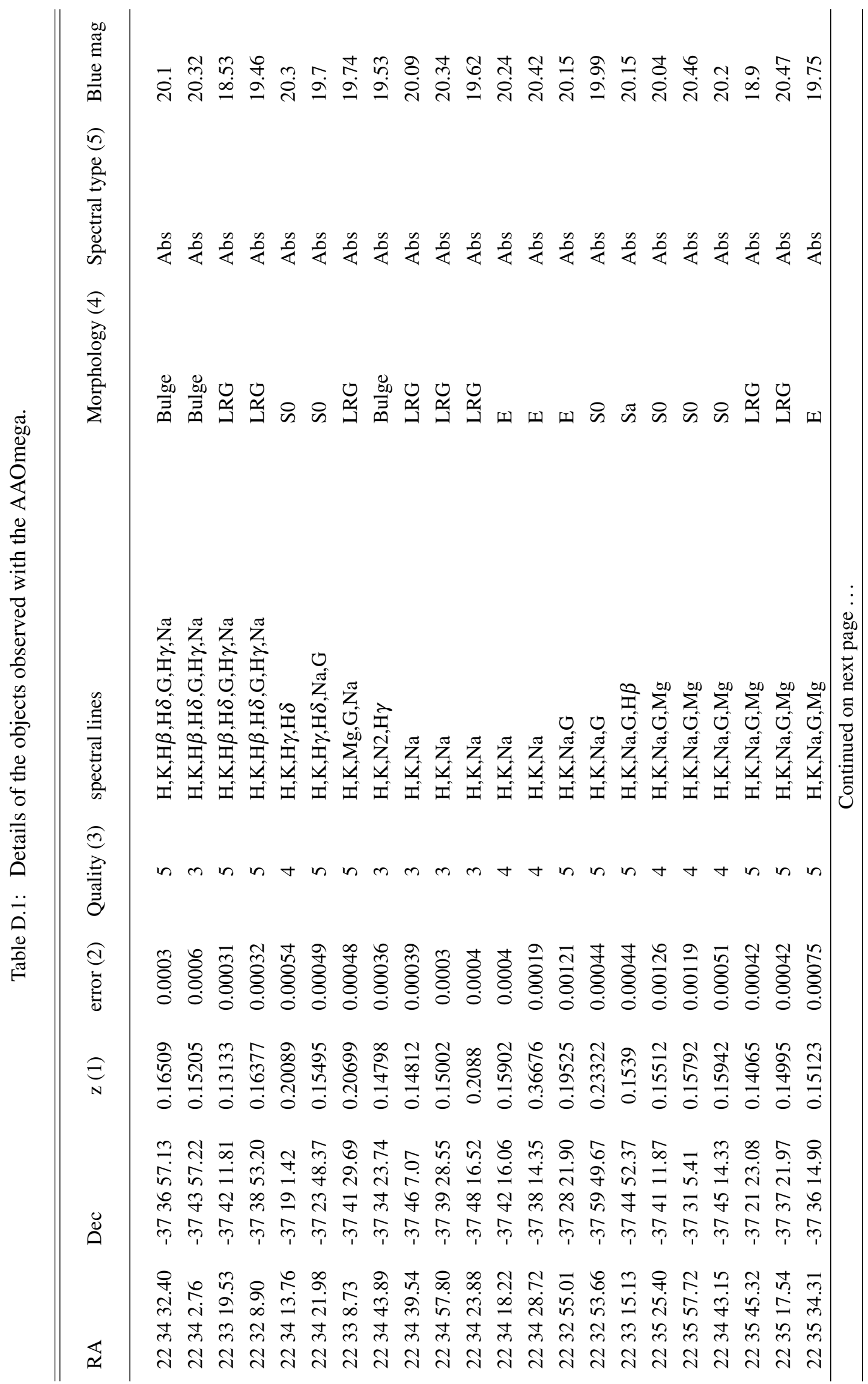




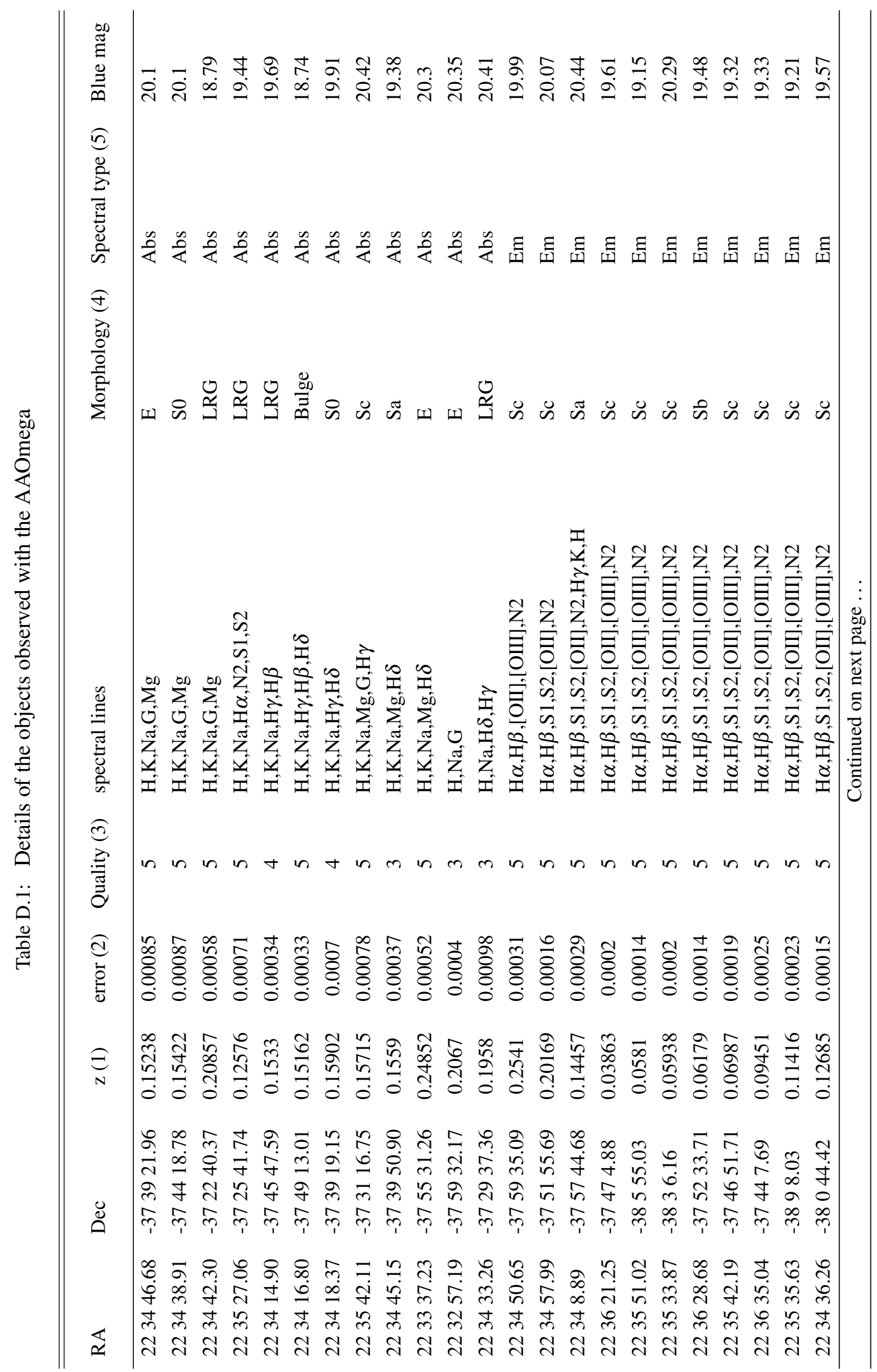




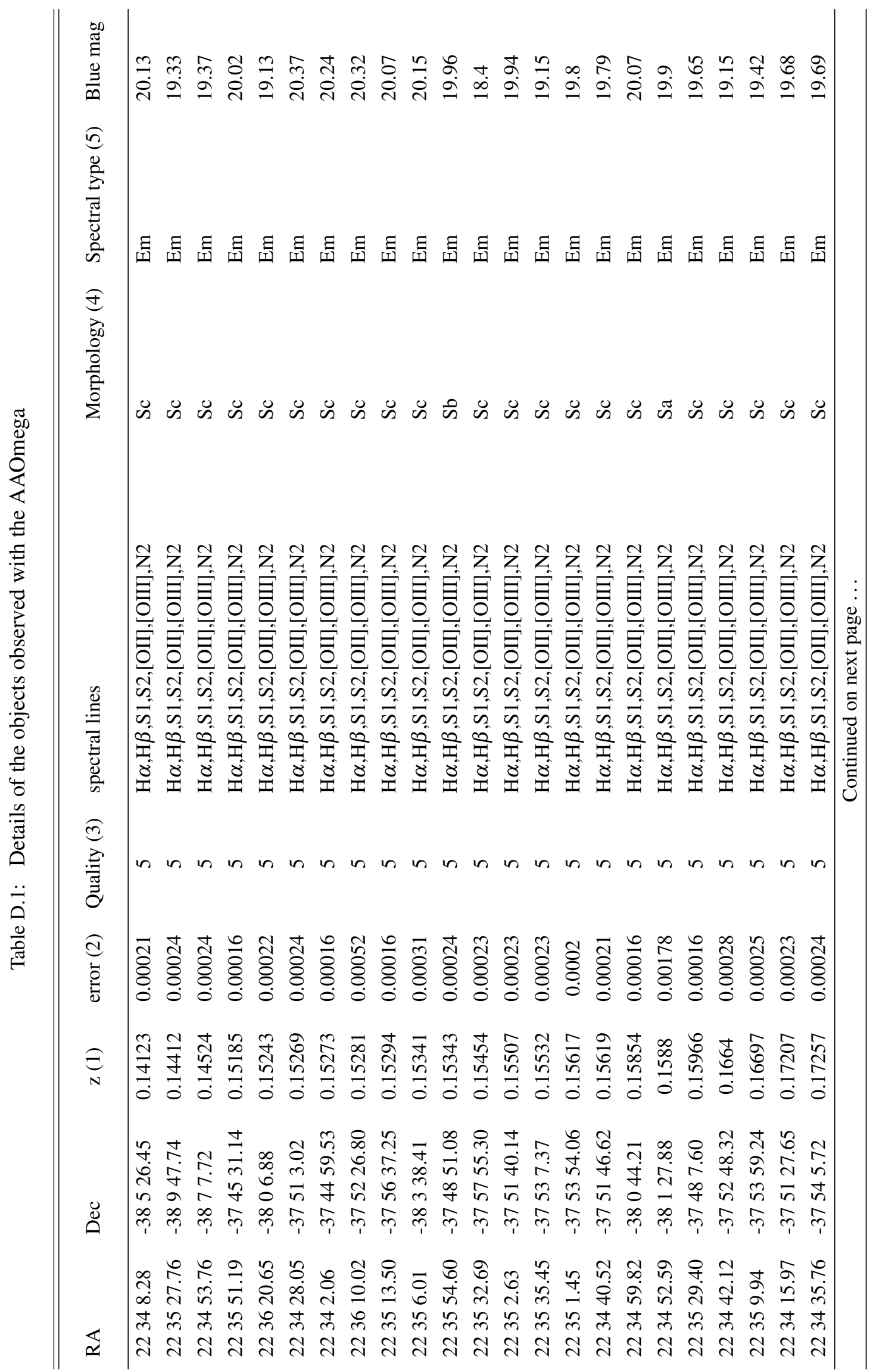




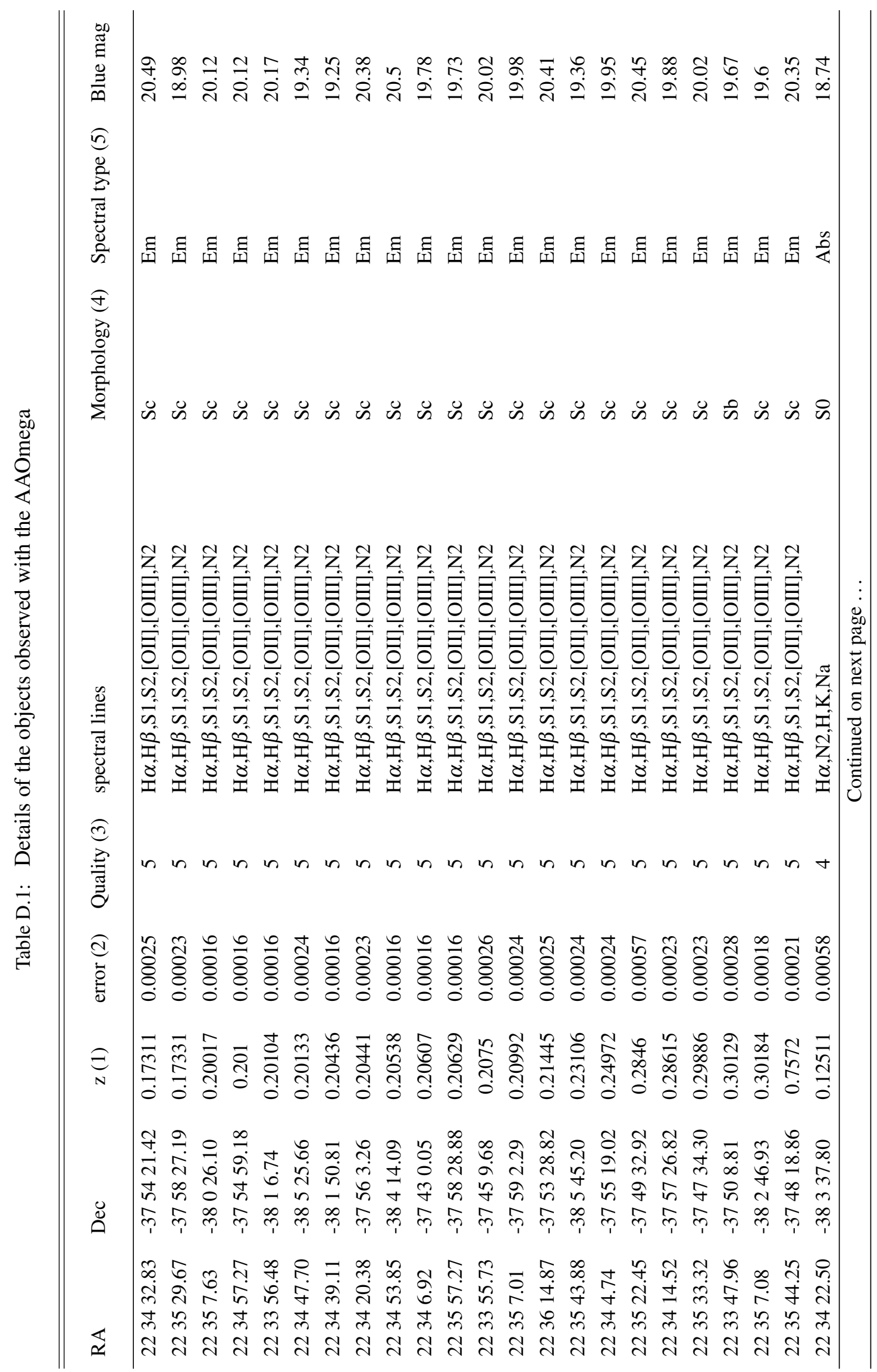




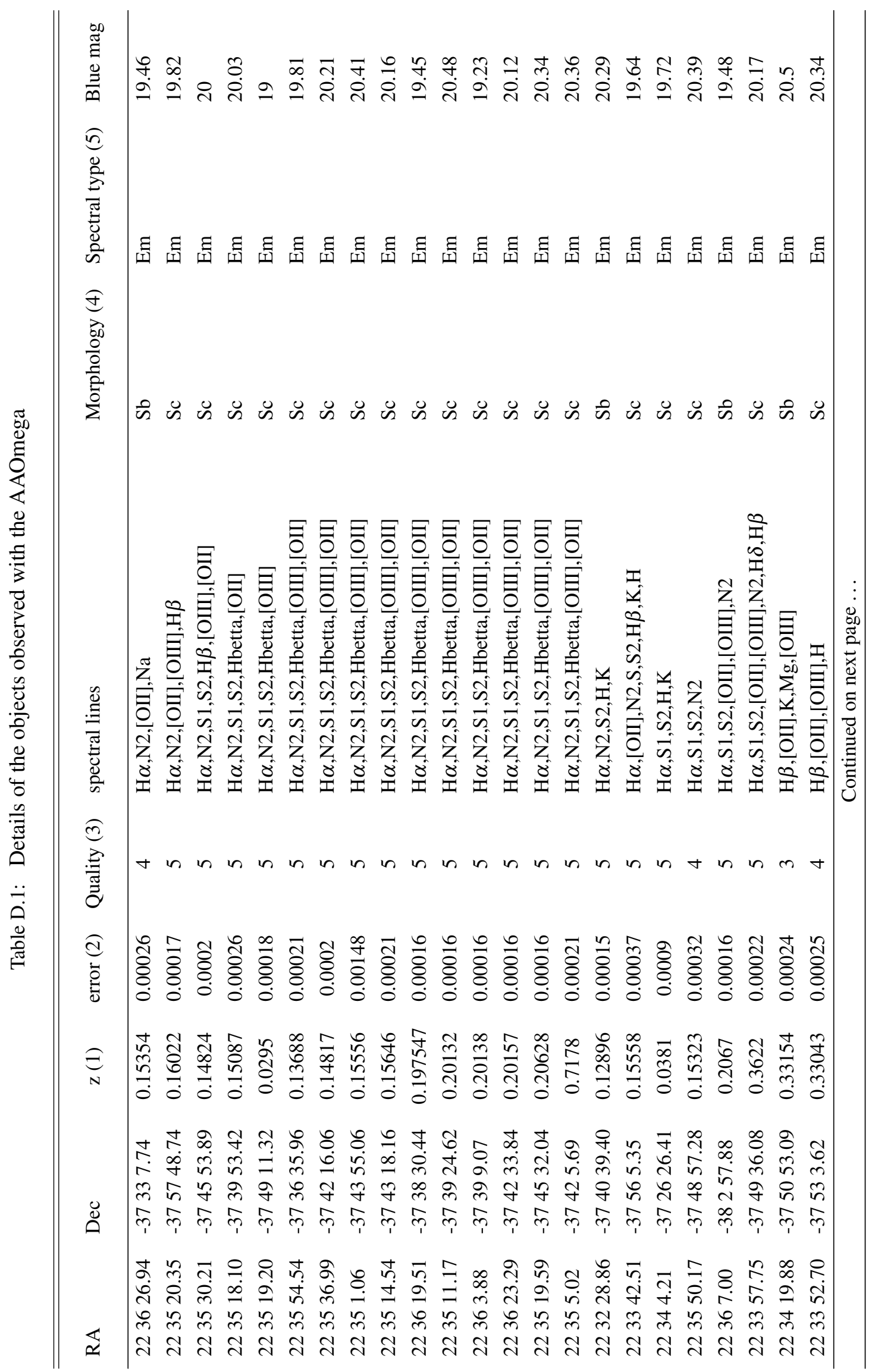




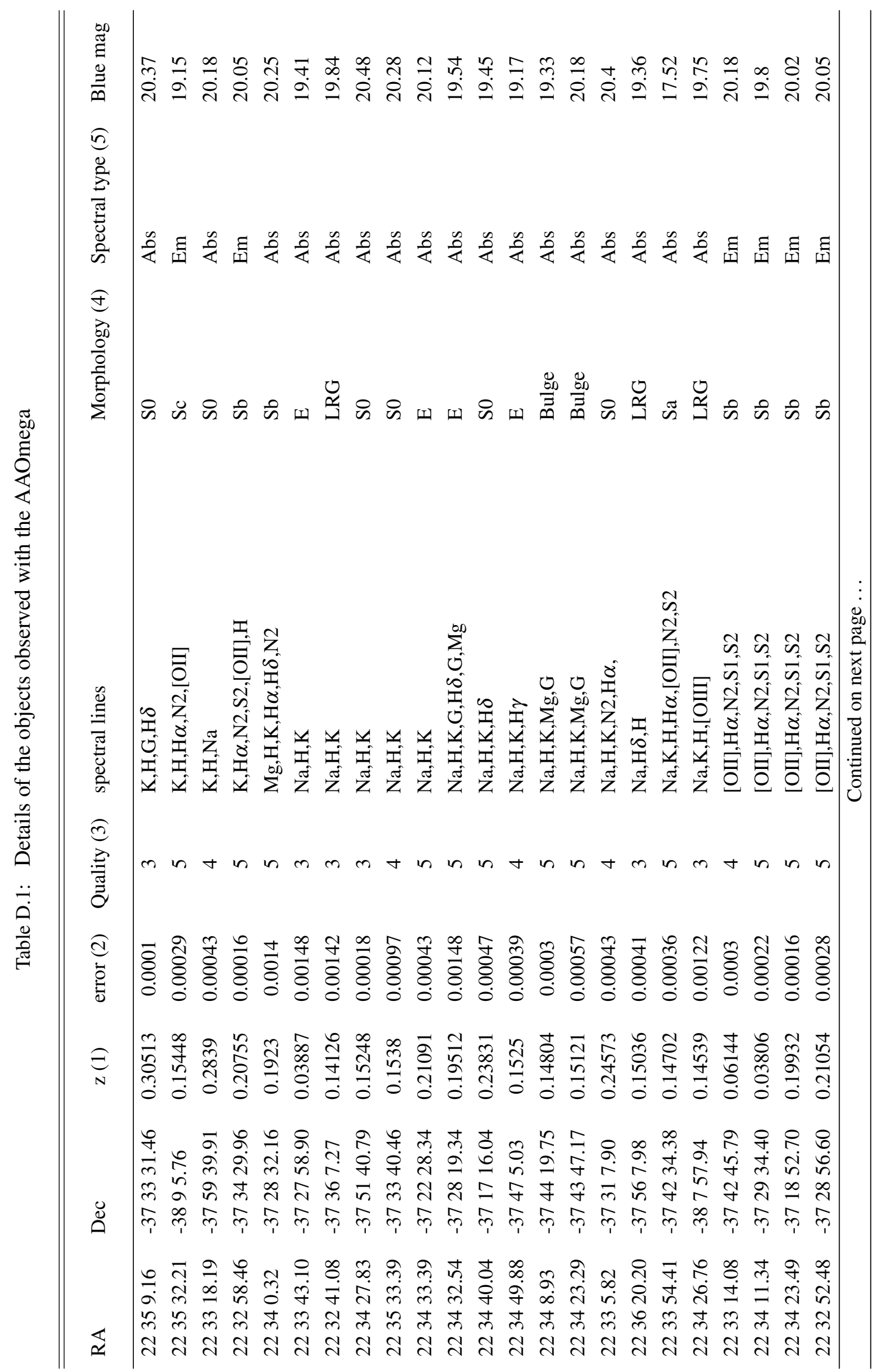




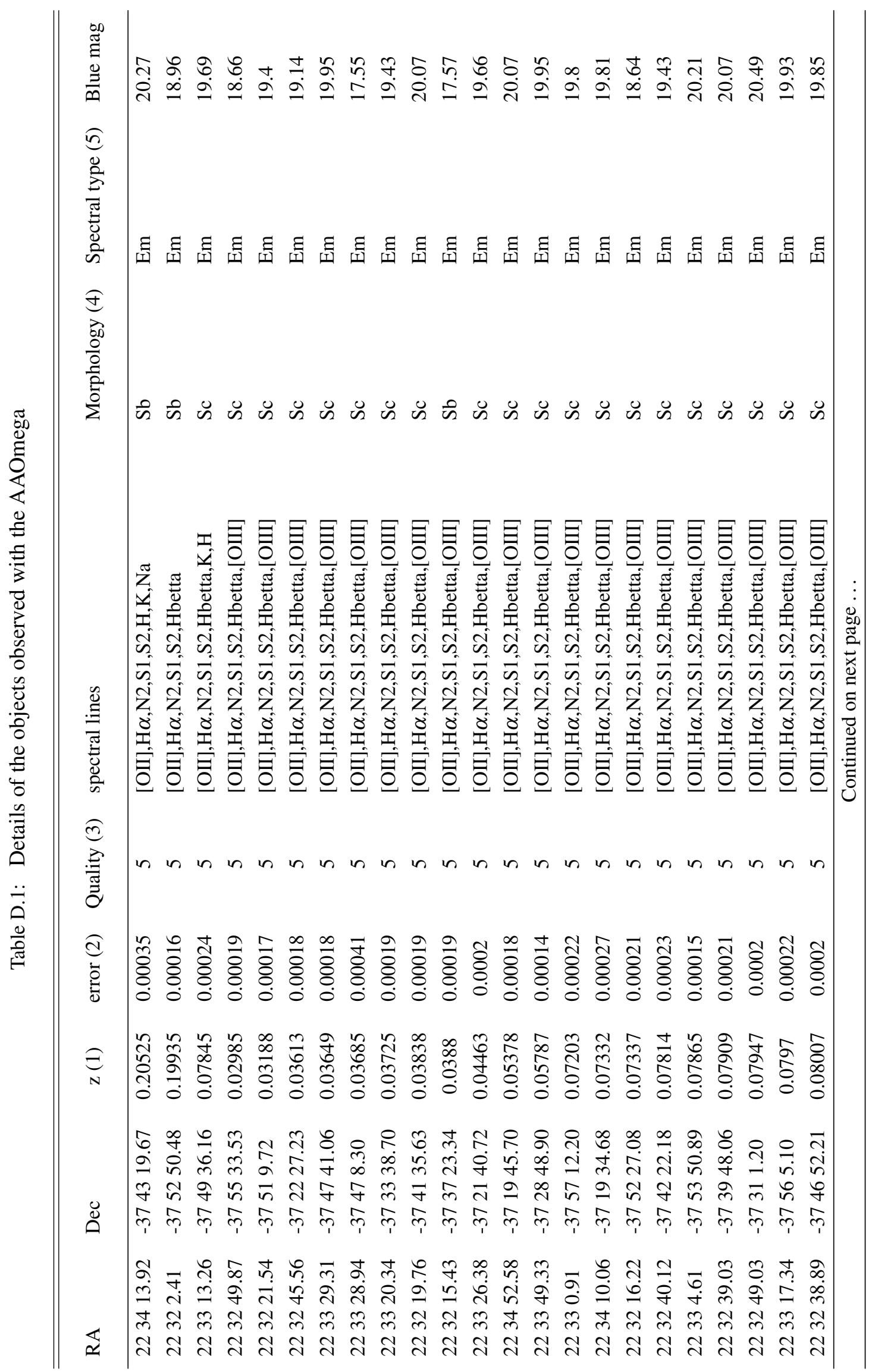




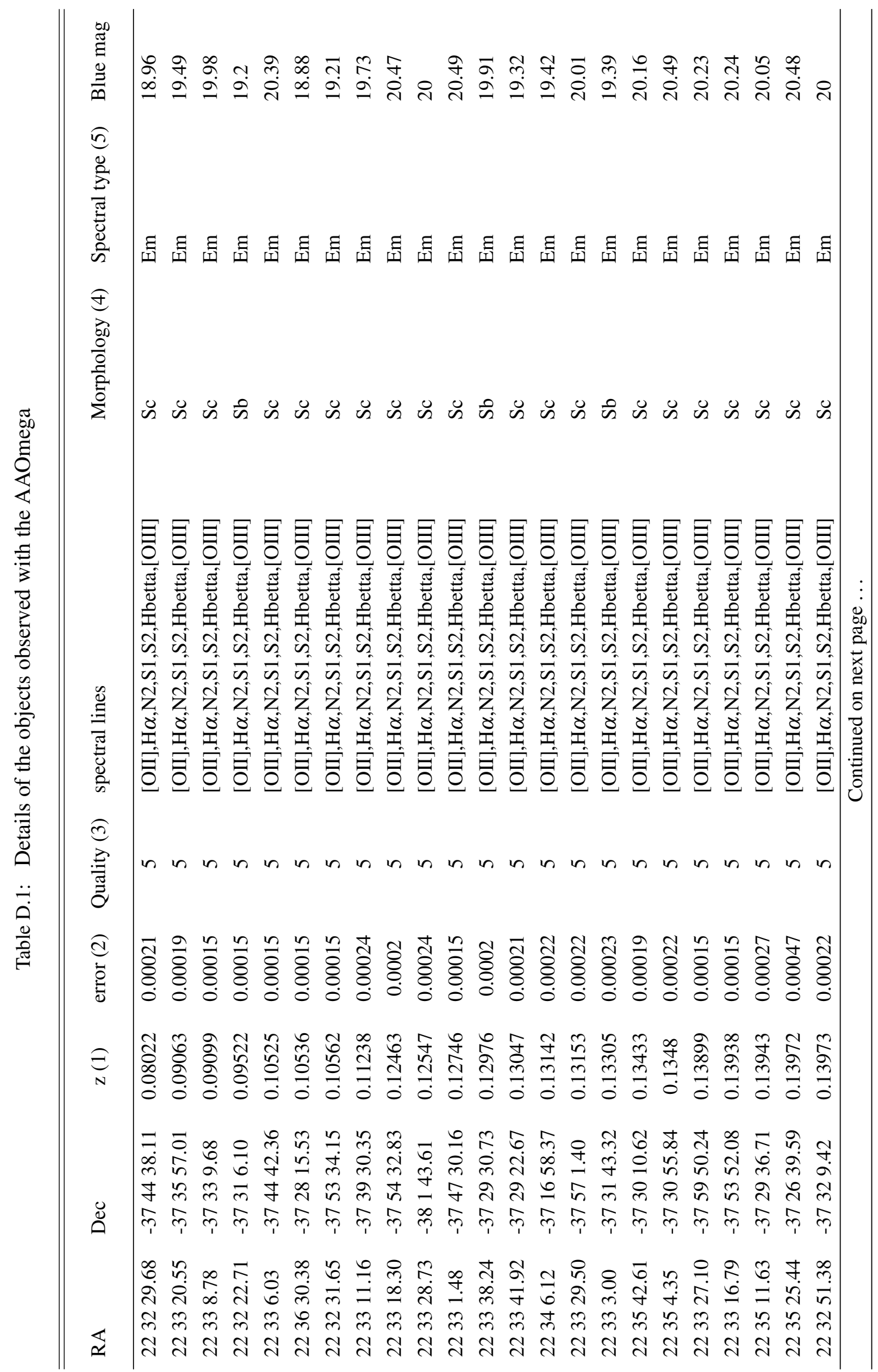




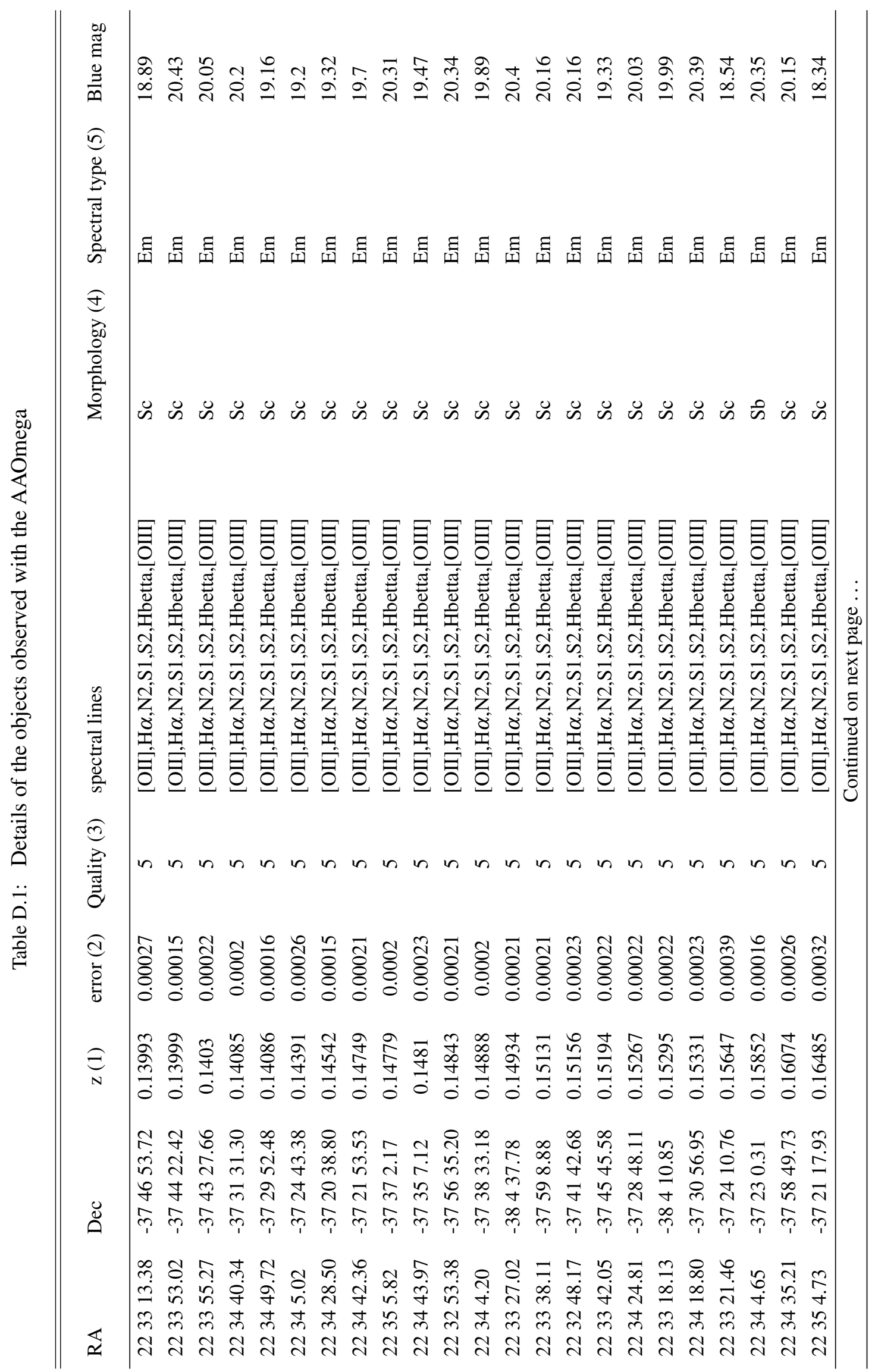




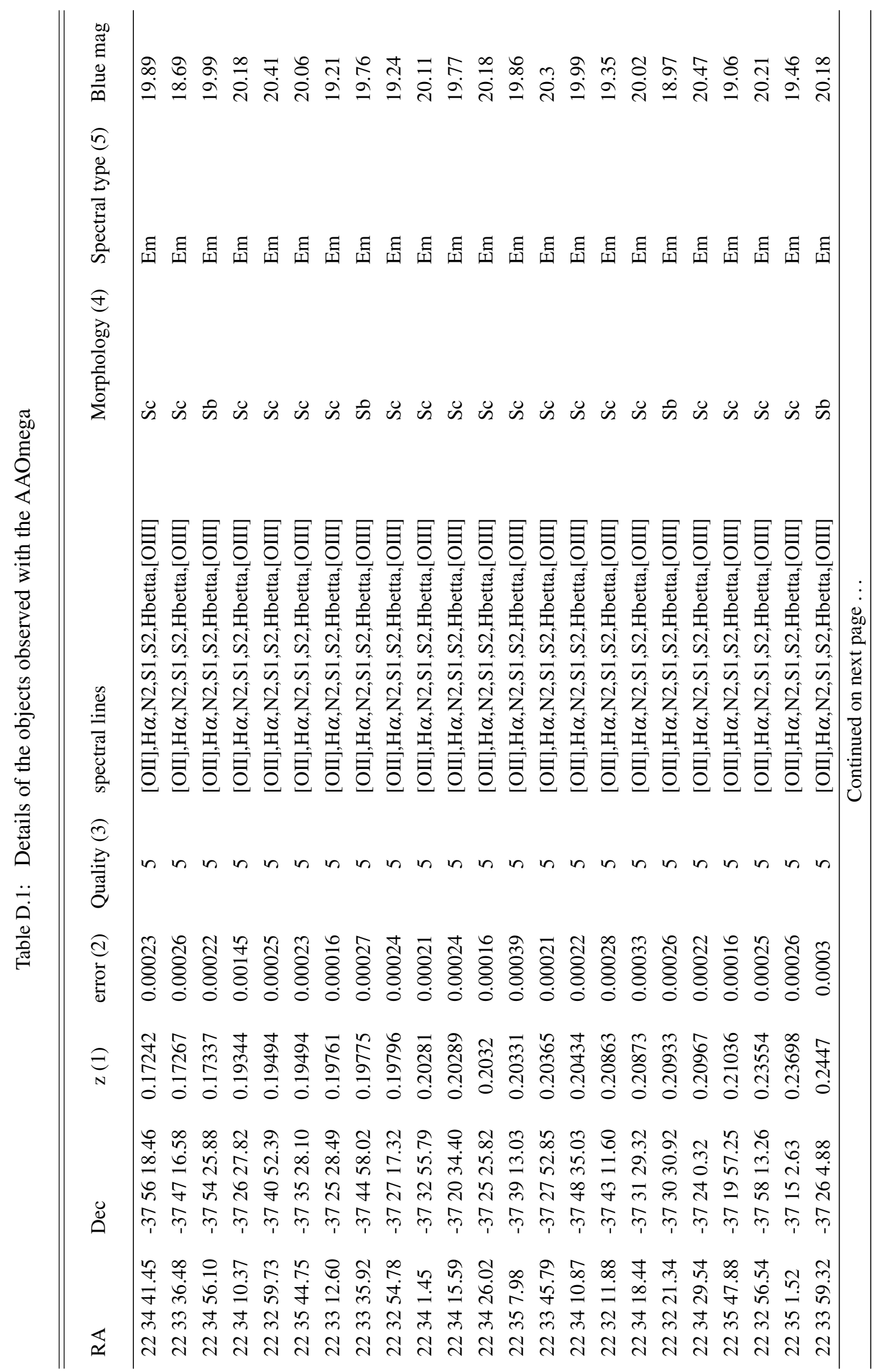




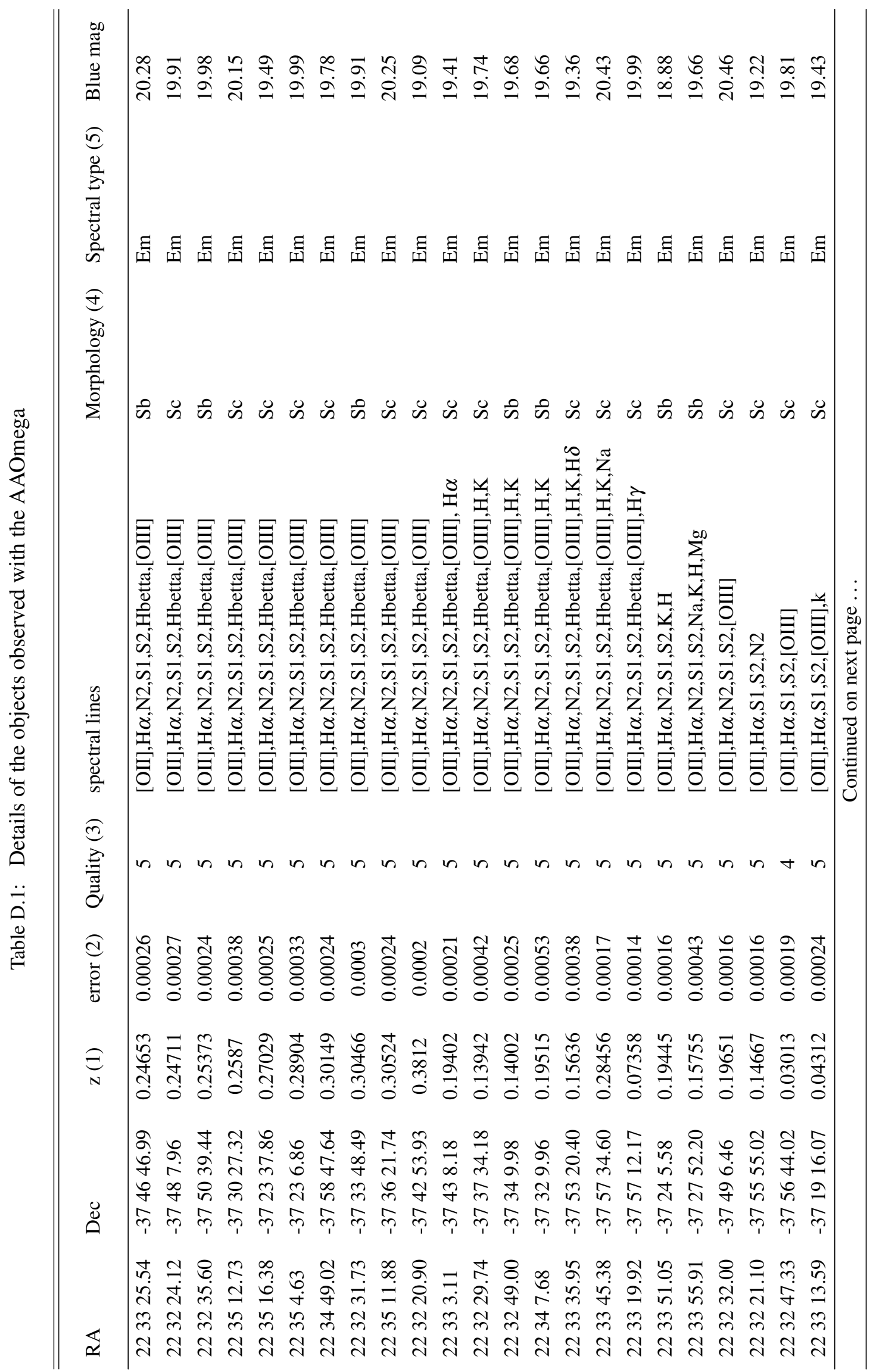




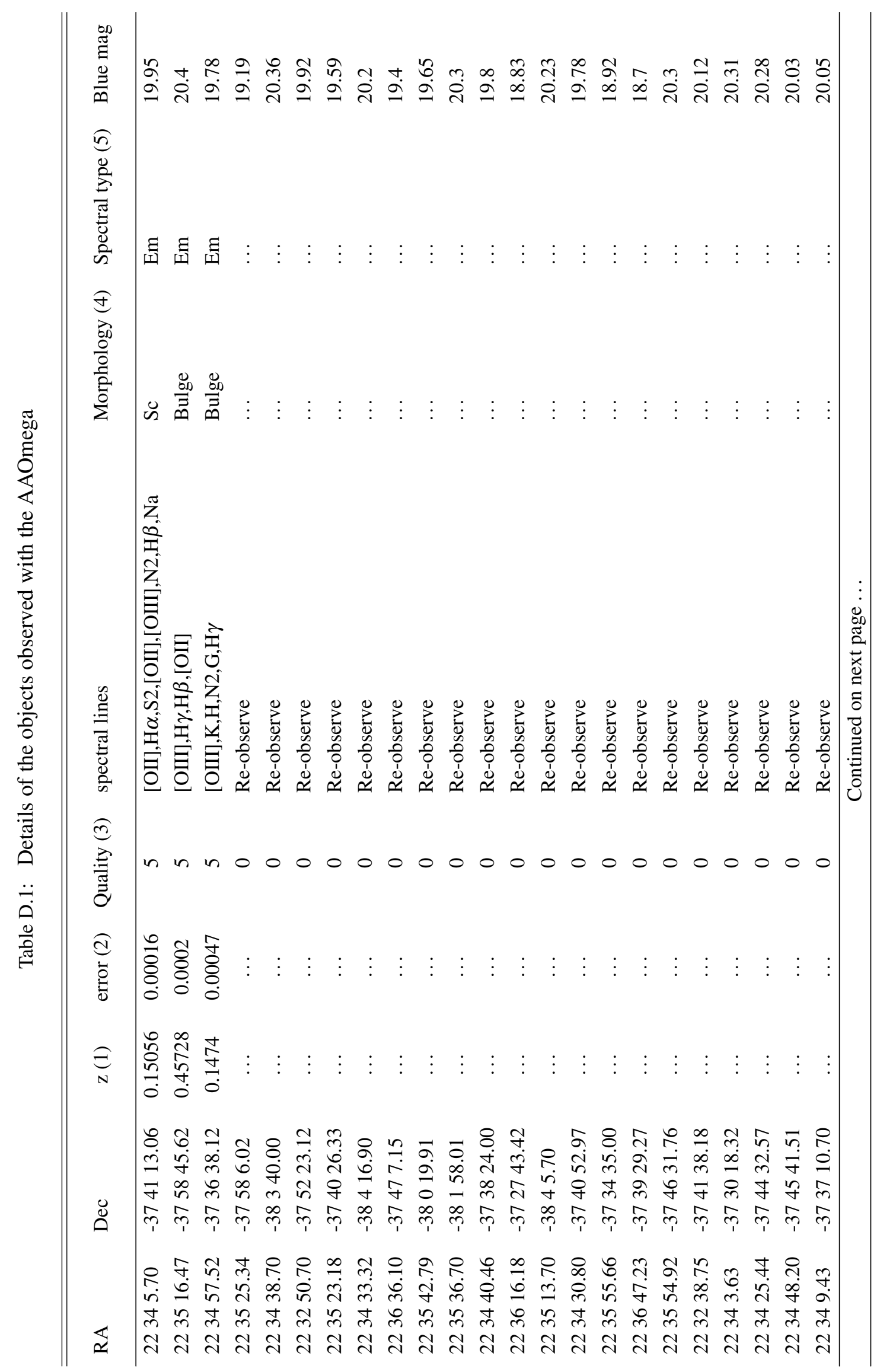




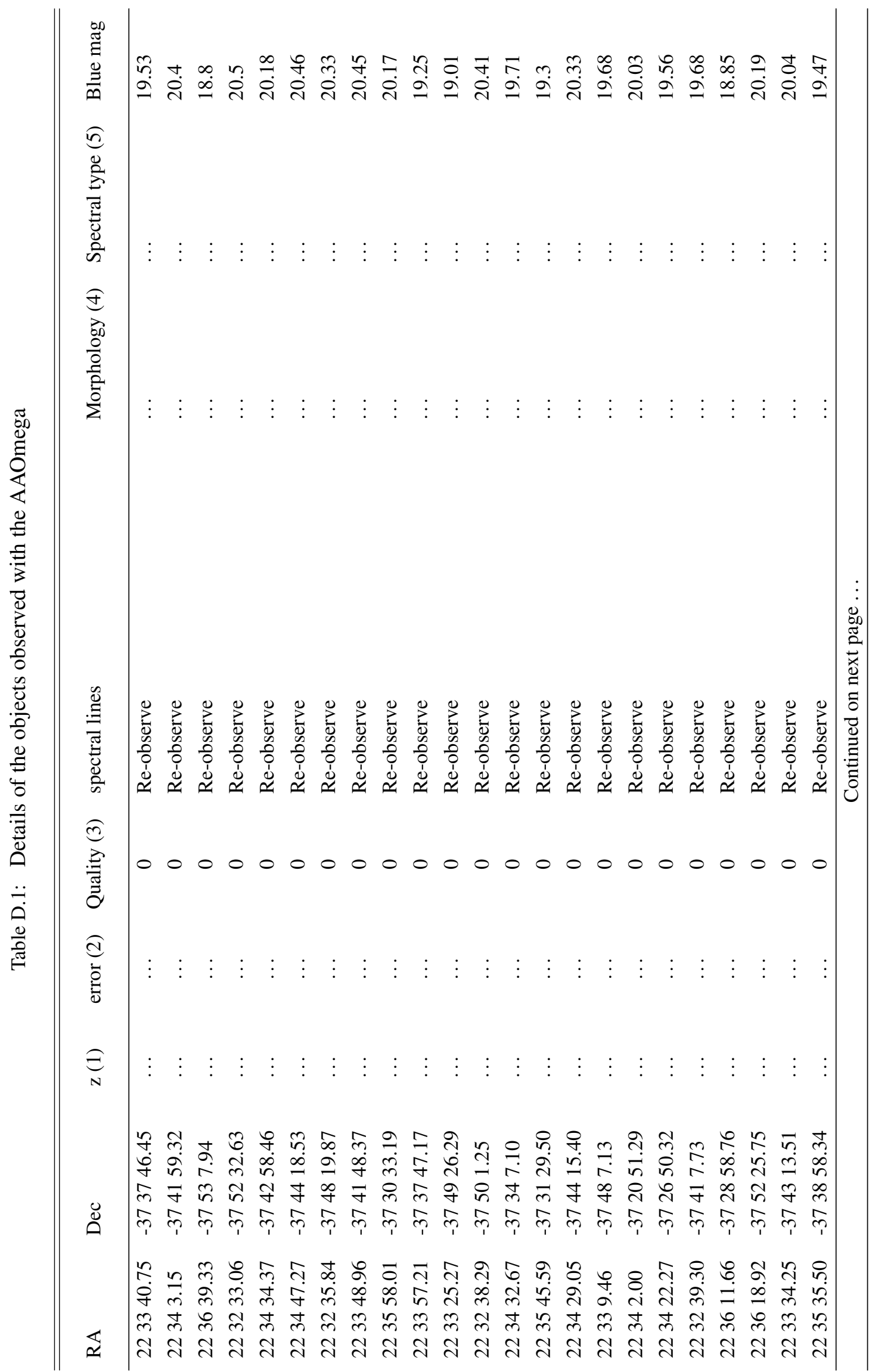




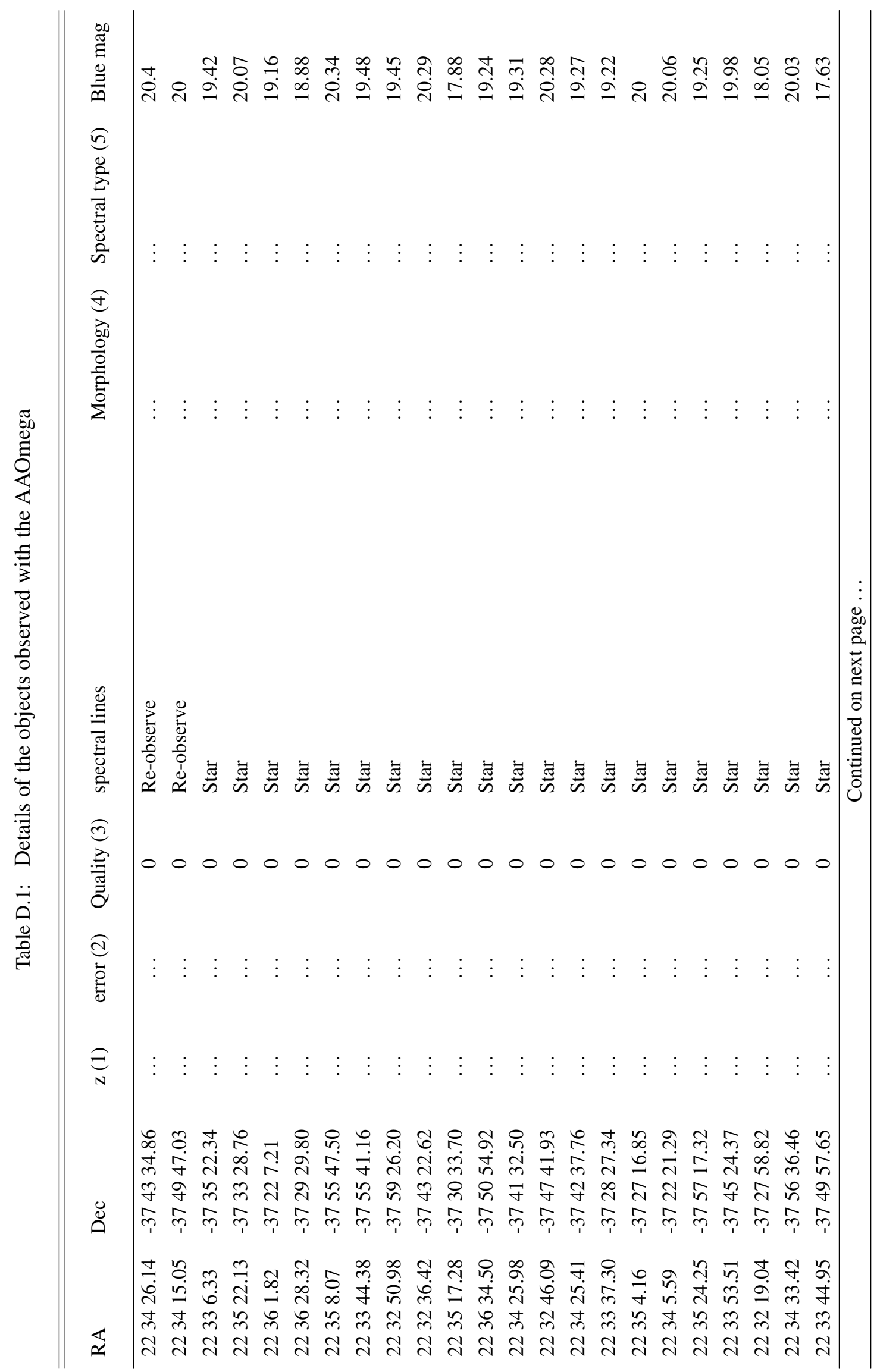




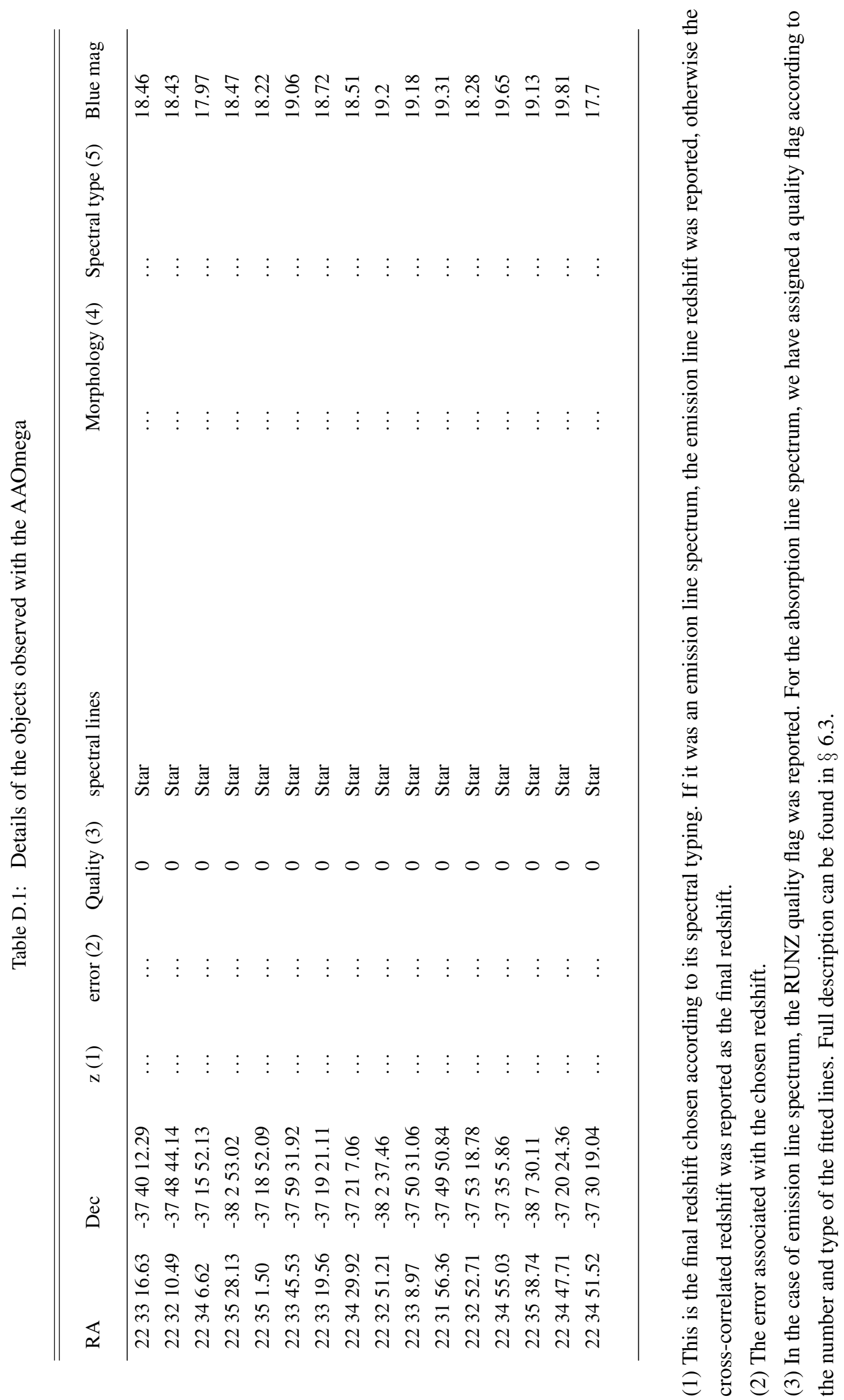




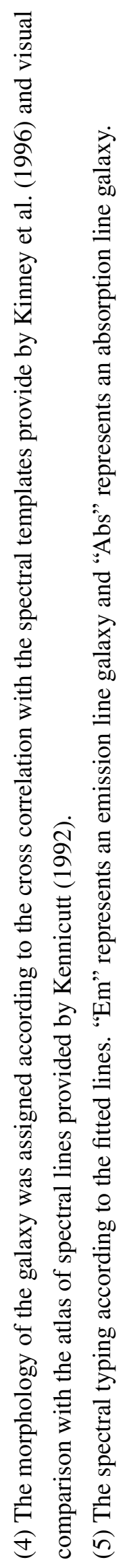



Table D.2: Table of the acronyms used in this thesis.

\begin{tabular}{cc}
\hline \hline Name & Acronym \\
\hline Australia Telescope Compact Array & ATCA \\
NASA/IPAC Extragalactic Database & NED \\
Giant Radio Halo & GRH \\
Giant Metrewave Radio Telescope & GMRT \\
GMRT radio Halo Survey & GRHGS \\
Extended GMRT radio Halo Survey & EGRHS \\
Green Bank Telescope & GBT
\end{tabular}

